
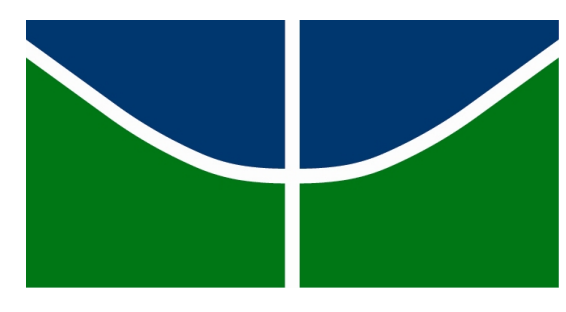

\author{
UNIVERSIDADE DE BRASÍLIA \\ INSTITUTO DE FÍSICA \\ PROGRAMA DE PÓS-GRADUAÇÃO EM FÍSICA
}

Análise de fenômenos devidos à modulação temporal em circuitos eletrodinâmicos quânticos dissipativos

Diego Silva Veloso

Orientador: Prof. Dr. Alexandre Dodonov

\author{
Brasília
}

julho de 2016 
Ficha catalográfica elaborada automaticamente, com os dados fornecidos pelo(a) autor(a)

Análise de Fenômenos devidos à modulação temporal em circuitos eletrodinâmicos quânticos dissipativos / Diego Silva Veloso; orientador Alexandre Dodonove. -- Brasília, 2016.

$111 \mathrm{p}$.

Dissertação (Mestrado - Mestrado em Física) - Universidade de Brasília, 2016.

1. EDQ de circuitos não-estacionários. 2. Efeito Casimir dinâmico e anti-efeito Casimir dinâmico. 3 . Equação mestra quântica. 4. Estados vestidos . 5 . Hamiltoniano de Rabi. I. Dodonove, Alexandre, orient. II. Título. 


\title{
"Análise de fenômenos devidos à modulação temporal em circuitos eletrodinâmicos quânticos dissipativos."
}

\author{
Por
}

\section{Diego Silva Veloso.}

Dissertação submetida ao Instituto de Física da Universidade de Brasília como parte dos requisitos para a obtenção do grau de Mestre em Física.

Aprovada por:

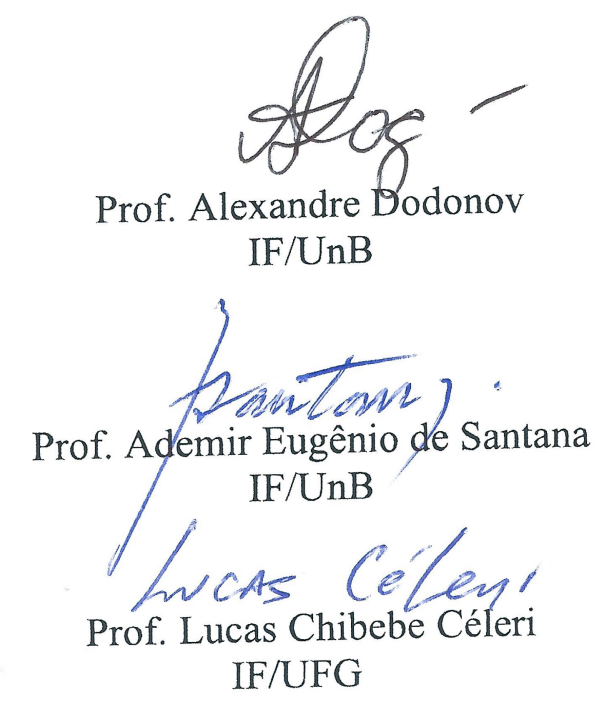

Prof. Dr. Fernando de Oliveira Albuquerque

Coordenador de Pós-Graduação

Instituto de Física 


\section{Resumo}

Neste trabalho, consideramos a arquitetura de Eletrodinâmica Quântica de Circuitos (EDQc), onde um átomo artificial de dois níveis interage com um único modo do campo eletromagnético quantizado confinado em um ressonador guia-de-onda sobre um chip supercondutor. Devido ao ambiente de alto controle em que a EDQc é formulada, consideramos modulações temporais periódicas de um ou mais parâmetros do sistema, impostas por um agente externo, caracterizando um sistema de EDQc não-estacionário. Um Hamiltoniano efetivo foi obtido para descrever a dinâmica do sistema e, de maneira mais intuitiva, caracterizar o efeito físico a ser tratado de acordo com a frequência de modulação imposta. Também descrevemos a dinâmica para modulações simultâneas e multifrequências. Entre os efeitos estudados estão os já conhecidos efeito Casimir dinâmico (ECD) e o comportamento anti-Jaynes-Cummings (AJC). Além desses efeitos, discutimos o regime anti-efeito Casimir dinâmico (AECD), que foi apresentado recentemente.

Em seguida, propomos duas abordagens para estudarmos os efeitos da dissipação Markoviana nas transições induzidas por modulação usando os estados vestidos da interação átomo-campo. A primeira abordagem foi através da equação mestra padrão da óptica quântica, e a segunda abordagem foi através da equação mestra na representação vestida, que foi recentemente apresentada. Nós analisamos como os diferentes canais dissipativos afetam a dinâmica do sistema e averiguamos a possibilidade de implementação dos fenômenos físicos estudados na arquitetura de EDQc. 


\section{Abstract}

In this work, we consider the circuit Quantum Electrodynamics architecture, where an artificial two-level atom interacts with a single mode of a quantized Eletromagnetic field confined in a waveguide resonator on a superconducting chip. Due to formulation of cQED in a highly controllable environment we consider that one or more system parameters undergo periodic time-modulation by an external agent. This regime characterizes the nonstationary circuit QED. We obtain an effective Hamiltonian to intuitively describe the dynamics of the system and characterize the physical effect to be treated according to the frequency of modulation. We describe the dynamics for a single-tone and a multi-tone modulations of one or multiple parameters. We study some previously known effects, such as the dynamical Casimir effect (DCE) and the anti-Jaynes-Cummings behavior. Besides these effects, we discuss the recently presented anti-dynamical Casimir effect (ADCE).

We employ two different approaches to asses the effects of Markovian dissipation on modulationinduced transitions between the atom-field dressed states. The first approach is the standard master equation of Quantum Optics and the second approach is through the recently presented dressedpicture master equation. We analyze how different dissipation channels affects the time evolution and verify the possibility of implementing of the studied physical phenomena in the cQED architecture. 


\section{Agradecimentos}

Agradeço à minha família por todo apoio emocional e afetuoso que me deram.

Agradeço ao professor Alexandre Dodonov por sua qualidade como líder e disposição em ensinar. Agradeço à pessoa Alexandre Dodonov por toda compreensão e boas conversas.

Agradeço aos amigos do convívio diário, especialmente àqueles que utilizaram seu precioso tempo com visitas ou mensagens de apoio quando estive longe deles.

Agradeço aos colegas físicos por toda ajuda.

Aos amigos que fiz na UnB por momentos de descontração nos corredores da universidade: muito obrigado.

Agradeço aos professores Arsen, Antony e Pinzul por me estimularem a estudar mais.

Por fim, à CAPES pelo suporte financeiro. 


\title{
Abreviaturas
}

\author{
AECD: Anti-efeito Casimir dinâmico
}

AJC: Anti-Jaynes-Cummings

DU: Dinâmica Unitária

DAP: Defasagem atômica pura

ECD: Efeito Casimir dinâmico

EDQ: Eletrodinâmica Quântica

EQCc: Eletrodinâmica Quântica de circuitos

EMP: Equação mestra padrão

EMEV: Equação mestra na representação de estados vestidos

EV: Estados Vestidos

EZE: Estado de zero excitação

RAP: Relaxação atômica pura

RD: Regime dispersivo

RPC: Relaxação pura da cavidade

RR: Regime ressonante

RWA: Rotating wave approximation (Aproximação de onda girante) 


\section{Lista de Figuras}

6.1 Geração de excitações a partir do EZE no regime ressonante. (a) Comportamento analítico aproximado para as populações. (b) Número médio de fótons criados sob RAP para a EMP (EMEV) em preto (vermelho). (c) Número médio de fótons obtidos a partir da solução analítica aproximada. (d) Probabilidade de excitação atômica para EMP (EMEV) em preto (vermelho). (e) Probabilidade de excitação atômica para solução analítica aproximada. (f) fator Q de Mandel. . . . . . . . . . . . . . . . . . . . . . 68

6.2 Fenômeno ECDg.(a) Número médio de fótons sem dissipação. (b) Comparativo númerico exato para o número médio de fótons sob relaxação atômica pura (preto) e relaxação pura da cavidade (vermelho). (c) Probabilidade de excitação atômica para RAP (RPC) em preto (vermelho). (d) Fator Q de Mandel para RAP (RPC) em preto(vermelho). . . . . . . . . .

6.3 Fenômeno anti-ECD sob dissipação via RAP. (a) Distribuição das populações ao longo do tempo adimensional $\tau^{\prime}=t \times|\theta|$. (b) Número médio de fótons criados e aniquilados em função do tempo. (c) Probabilidade de excitação atômica. (d) Fator $Q$ ao longo do tempo adimensional $\tau^{\prime}$

6.4 Fenômeno anti-ECD: solução exata para EMP. (a) Número médio de fótons na ausência (presença) de dissipação em preto (vermelho). (b) Probabilidade de excitação atômica $P_{e}$ sem (com) a presença de dissipação em preto (vermelho). (c) Fator $Q$ em função do tempo sem (com) a presença de dissipação em preto (vermelho). (d) Probabilidade para o estado atômico fundamental sem (com) efeitos dissipativos em preto (vermelho) . . . . . . . . . . . 72 
6.5 Dupla modulação da frequência no regime ressonante. (a) Número médio de fótons sob dissipação para a EMP (EMEV) em preto (vermelho).(b) Fator Q para EMP (EMEV) em preto (vermelho). (c) Probabilidade de excitação atômica $P_{e}$ descrita pela EMP (EMEV) em preto (vermelho). . . . . . . . . . . . . . . . . . . . . 74

6.6 Regime anti-Jaynes-Cummings. (a) Comportamento das populações em função do tempo adimensional $\tau^{\prime}=t \times|\theta|$ sob dissipação gerada por RAP. (b) Número médio de fótons criados sem dissipação em preto e com dissipação analisada para EMP (EMEV) em azul (vermelho). (c) Comportamento das populações em função do tempo adimensional $\tau^{\prime}$ sob dissipação gerada por RCP. (d) Probabilidade de excitação atômica sem dissipação em preto e com dissipação analisada para EMP (EMEV) em azul (vermelho). (e) Número médio de fótons gerados sob a presença de dissipação analisada para RAP (RPC) em preto (vermelho). (f) Fator $Q$ sem dissipação na linha preta e com dissipação analisada para EMP (EMEV) em azul (vermelho). . . . . . . . . . . . . . . . . . . . . 76

6.7 Dupla modulação da frequência: regime AECD incrementado. (a) Número médio de fótons sem (com) a presença de dissipação em preto (vermelho). (b) Probabilidade de excitação atômica sem (com) a presença de dissipação em preto (vermelho). (c) Fator Q sem (com) a presença de dissipação em preto (vermelho). (d) Probabilidade para o estado atômico fundamental sem (com) a presença de dissipação em preto (vermelho). . . . . . . . . . . . 78

6.8 Dupla modulação da frequência: regime AECD incrementado. Taxas de dissipação moderadas para EMP. (a) Número médio de fótons sem (com) a presença de dissipação em preto (vermelho). (b) Probabilidade de excitação atômica sem (com) a presença de dissipação em preto (vermelho). (c) Fator Q sem (com) a presença de dissipação em preto (vermelho). (d) Probabilidade para o estado atômico fundamental sem (com) a presença de dissipação em preto (vermelho). . . . . . . . . . . . . . . . . . . . 79 


\section{Conteúdo}

1 Introdução $\quad 2$

2 Descrição analítica $\quad 6$

2.1 Solução Geral . . . . . . . . . . . . . . . . . . . . . . . . . . . . 7

2.2 Efeitos físicos devidos a modulações ressonantes $\ldots \ldots \ldots \ldots \ldots$

3 Equação Mestra na representação dos Estados Vestidos 12

3.1 Formalismo matemático para construção da Equação Mestra . . . . . . . . . . . . . . 12

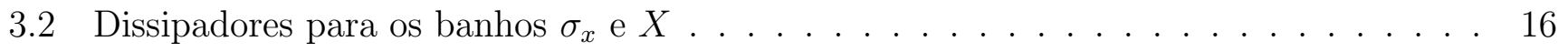

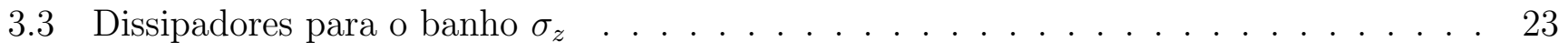

3.3 .1 Modelo clássico . . . . . . . . . . . . . . . . . . . . . . . . . 23

3.3 .2 Modelo Quântico . . . . . . . . . . . . . . . . . . . . . . . . . 25

4 Considerações sobre a Equação Mestra Padrão 32

4.1 Equação Mestra Padrão . . . . . . . . . . . . . . . . . . . . . . . . . 35

4.2 Simplificações para EMP . . . . . . . . . . . . . . . . . . . 36

5 Comportamento assintótico $\quad 40$

5.1 Comportamento para duas excitações . . . . . . . . . . . . . . . . . . . . . 41

5.1 .1 Geração de pares de excitações no regime ressonante . . . . . . . . . . . . . 41

5.1 .2 Regime anti-Jaynes-Cummings (AJC) . . . . . . . . . . . . . 45

5.1 .3 Regime anti-efeito Casimir dinâmico (AECD) . . . . . . . . . . . . 52

5.2 Comportamento para quatro excitações . . . . . . . . . . . . . . . . . 55

5.2.1 Geração de fótons do vácuo incrementada no regime ressonante . . . . . . . . 55 
5.2 .2 Regime anti-ECD incrementado . . . . . . . . . . . . . . . 60

5.3 Efeito Casimir Dinâmico fundamental (ECDg) . . . . . . . . . . . . . . . 63

6 Análise numérica $\quad 66$

$\begin{array}{llc}7 & \text { Conclusões e Perspectivas } & 81\end{array}$

$\begin{array}{lc}\text { A Operador Paridade } & 83\end{array}$

B RWA $\quad 85$

C Densidade Espectral $\quad 88$

C.1 Transformada de Fourier . . . . . . . . . . . . . . . . . . . . . . . . . . . . . . 89

C.2 Densidade espectral de uma função periódica . . . . . . . . . . . . . . . . . . . . 90

C.3 Densidade espectral de um processo randômico . . . . . . . . . . . . . . . . . . . 92

D Estados Vestidos e autovalores de Jaynes-Cummings $\quad 94$ 


\section{Capítulo 1}

\section{Introdução}

A Eletrodinâmica Quântica (EDQ) de circuitos não-estacionários tem sido uma das áreas de pesquisa mais ativas nos últimos anos. Eles consistem de "átomos artificias" mesoscópicos supercondutores interagindo com um campo eletromagnético confinado em um ressonador guia-de-onda [1-3] sob condições não-estacionárias impostas por um agente externo. Os estudos de circuitos de Eletrodinâmica Quântica derivam da bem-sucedida realização em sistemas de estado-sólido, ampliando a área tradicional de EDQ de cavidades ${ }^{1}$ [4-7]. Na construção de circuitos de EDQ, utiliza-se de junções Josephson, que sob certas condições podem formar átomos artificiais com níveis discretos de energia (ou qubits supercondutores, como ficaram conhecidos) [2,4]. Como ramo do estado sólido, o regime não-estacionário de circuitos quânticos supercondutores vem à luz dada a possibilidade inédita de modulações temporais de parâmetros do sistema, permitidas pelo ambiente altamente controlável de estado sólido. Entre os parâmetros citados estão a frequência da cavidade, frequências de transições atômicas (no caso de diversos níveis) e força de acoplamento átomo-campo, todos ajustados como funções temporais controladas externamente [3,13].

Entretanto, qualquer sistema físico aberto evolui de maneira irreversível, estando qualquer observável ou controle sujeitos à decoerência em virtude de defasagem ou relaxação. Esses fenômenos foram objetos de estudo na Mecânica Quântica desde os primórdios, podendo-se citar L. Landau e W. Pauli como alguns de seus precursores $[14,15]$. De forma direta, isso atribui-se a um ambiente que drena a informação de um sistema quântico, crescendo, então, sua entropia durante a evolução.

\footnotetext{
${ }^{1}$ Os termos "cavity Quantum Electrodynamics" e "circuit Quantum Electrodynamics" foram traduzidos livremente para Eletrodinâmica Quântica de cavidades e Eletrodinâmica Quântica de circuitos, respectivamente.
} 
No estudo desses problemas é interessante dividir o sistema total em duas partes: o sistema $\mathcal{S}$ de interesse em particular e o ambiente, usualmente definido como reservatório $\mathcal{R}$, com um número muito grande de graus de liberdade, como salienta W. Vogel e D.-G. Welsh [19]. Como o sistema é alterado, ele será também chamado de sistema dinâmico, enquanto o ambiente é tratato como sistema dissipativo, banho (térmico) ou reservatório.

Essa dinâmica irreversível tem sido verificada em EDQ de cavidades há algumas décadas com a observação de decoerência na evolução de superposições mesoscópicas de estados quânticos, por exemplo [11]. Mais recentemente, circuitos supercondutores quânticos têm sido estudados de forma detalhada oferecendo grandes contribuições a respeito de processos de relaxação e defasagem $[1,3]$. Estudos teóricos ajudaram a esclarecer o impacto da medição em processos de defasagem em um qubit supercondutor na arquitetura de EDQ de circuitos [10]. Outros efeitos dissipativos aos quais a arquitetura de EDQ de circuitos está sujeita, em particular, a relaxação atômica e relaxação pura da cavidade, são afirmados como aspectos importantes a serem analisados [16].

O Efeito Casimir Dinâmico (ECD) é um dos maiores expoentes do sucesso desse ramo de pesquisa em EDQ de circuitos [36]. Como sabido, o ECD [39] é um fenômeno não-estacionário caracterizado pela criação de fótons a partir do estado fundamental ou um estado inicial qualquer do sistema. A ocorrência do fenômeno se dá com a rápida mudança na geometria da cavidade ou propriedades materiais das fronteiras ou meio dentro da cavidade. Em alguns trabalhos voltados à geração de fótons em circuitos de Eletrodinâmica Quântica não-estacionários estão inclusos os efeitos de dissipação, como nos trabalhos [7, 12,27], ou recentemente em estudos relacionados ao Efeito Casimir Dinâmico [25], todavia, a maioria dos estudos levam em consideração apenas a análise da dinâmica unitária para diferentes regimes de parâmetros e formas de modulação.

Recentemente $[42,43]$, foi mostrado que a modulação temporal de parâmetros do sistema usando perturbação harmônica simples ou multi-frequência (compreendendo duas ou mais funções do tempo harmônicas) faz surgir novos tipos de interações efetivas [41]. Para isso, uma equação diferencial piloto foi deduzida sob uma série de aproximações para descrever a evolução temporal das amplitudes de probabilidade. A partir desta equação é possível descrever o comportamento do sistema não-estacionário em resposta a modulações harmônicas ressonantes. Porém, tal formulação analítica foi somente para sistemas fechados, na ausência de perdas. Diante disso, é de grande interesse analisar como dissipação altera fenômenos de modulação temporal de parâmetros ligados à 
arquitetura de EDQ de circuitos mais sofisticados [38]. Para isto, no capítulo 2, será proposto um Hamiltoniano efetivo aproximado que fornece a mesma física que equação diferencial piloto, porém simplifica enormemente a notação um tanto complicada apresentada em [43]. Assim, a partir das modulações fornecidas em [43], os fenômenos de criação ou aniquilação de excitações a serem analisados serão: Efeito Casimir dinâmico, geração de pares de excitações do vácuo no regime ressonante, comportamento anti-Jaynes-Cummings (AJC), anti-efeito Casimir dinâmico (AECD) e, por último, a incrementação via modulação em dupla-frequência.

O objetivo do trabalho é investigar quais fenômenos podem ser implementados experimentalmente com a tecnologia atual e compreender como os diferentes canais de dissipação afetam a dinâmica. Para isso, dois modelos Markovianos à temperatura zero serão propostos a fim de contabilizar efeitos dissipativos, seguindo a abordagem de [19,20]. É bem conhecido que os sistemas quânticos abertos podem ser descritos pela equação mestra abaixo, onde $\hat{\mathcal{L}}$ é o superoperador Liouvilliano que descreve os efeitos dissipativos. A primeira abordagem vai considerar que o qubit e a cavidade são independentes para se chegar a uma expressão mais simples do Liouvilliano. A equação

$$
\frac{d \hat{\rho}}{d t}=-i[\hat{H}, \hat{\rho}]+\hat{\mathcal{L}} \hat{\rho}
$$

onde $\hat{H}$ descreve o Hamiltoniano do sistema, será utilizada a fim de se saber como a dinâmica unitária é modificada na presença de decoerência. Serão deduzidos valores assintóticos de várias observáveis e, em seguida, a dinâmica será comparada numericamente com a segunda abordagem. Na Equação Mestra Padrão $[19,20,30,31,40]$ obtida sob aproximação de Markov o kernel dissipativo é

$$
\hat{\mathcal{L}}_{E M P} \bullet \kappa \hat{\mathcal{D}}[\hat{a}] \bullet+\gamma \hat{\mathcal{D}}\left[\hat{\sigma}_{-}\right] \bullet+\frac{\gamma_{\phi}}{2} \hat{\mathcal{D}}\left[\hat{\sigma}_{z}\right] \bullet,
$$

onde $\hat{a}\left(\hat{\sigma}_{-}\right)$é o operador abaixamento da cavidade (do átomo) e $\hat{\sigma}_{z}$ é o operador de inversão atômica. As taxas de relaxação atômica e da cavidade são $\gamma$ e $\kappa$, respectivamente, enquanto $\gamma_{\phi}$ refere-se à taxa de defasagem atômica pura. $\hat{\mathcal{D}}[\hat{O}] \hat{\rho}=\frac{1}{2}\left(\hat{O} \hat{\rho} \hat{O}^{\dagger}-\hat{O}^{\dagger} \hat{O} \hat{\rho}-\hat{\rho} \hat{O}^{\dagger} \hat{O}\right)$ é conhecido como superoperador Lindbladiano estudado em [21], embora sua estrutura geral (preservando a hermiticidade, normalização e positividade de $\hat{\rho}$ ) tenha sido deduzida por outros autores anteriormente, por exemplo [22]. No capítulo 4 mais considerações serão feitas.

A outra abordagem levará em conta a Equação Mestra apresentada mais recentemente em [16], que leva em consideração o acoplamento qubit-ressonador. Isto se difere drasticamente 
da Equação Mestra Padrão, onde havia um único canal de dissipação para cada subsistema. Os autoestados que incluem tal acoplamento são os Estados Vestidos, definidos e explicados no apêndice D. A frequência $\Delta_{k j}=\lambda_{k}-\lambda_{j}$ se refere à frequência de transição entre autoestados $|j\rangle \leftrightarrow|k\rangle$ e tais transições incoerentes ocorrem a taxas que dependem da densidade espectral dos banhos àquelas frequências. Sua dedução será realizada capítulo 3 e sua parte dissipativa será dada pela ação do superoperador Liouvilliano

$$
\mathcal{L}_{E V} \bullet \hat{\mathcal{D}}\left[\sum_{j} \Phi(j)|j\rangle\langle j|\right] \bullet+\sum_{j, k \neq j} \Gamma_{\phi}(j, k) \hat{\mathcal{D}}[|j\rangle\langle k|] \bullet+\sum_{j, k>j} \Gamma(j, k) \hat{\mathcal{D}}[|j\rangle\langle k|] \bullet
$$

Na expressão acima, os parâmetros $\Gamma_{\kappa}(j, k)=\kappa\left(\Delta_{k j}\right)\left|X^{j k}\right|^{2}$ e $\Gamma_{\gamma}(j, k)=\gamma\left(\Delta_{k j}\right)\left|\sigma_{x}^{j k}\right|^{2}\left(\operatorname{sendo} X^{j k}=\right.$ $\langle j|X| k\rangle$ e $\sigma_{x}^{j k}=\left\langle j\left|\sigma_{x}\right| k\right\rangle, X=\left(\hat{a}+\hat{a}^{\dagger}\right)$ e $\left.\sigma_{x}=\left(\sigma_{-}+\sigma_{+}\right)\right)$referem-se ao ressonador e o qubit, sendo $\kappa\left(\Delta_{k j}\right)$ e $\gamma\left(\Delta_{k j}\right)$ suas taxas de dissipação, respectivamente. O termo $\Gamma(j, k)=\Gamma_{\kappa}(j, k)+\Gamma_{\gamma}(j, k)$ serve para compactar a notação dos termos para as taxas de relaxação. Como será visto mais adiante, $\Phi(j)=\sqrt{\frac{\gamma_{\phi}(0)}{2}} \sigma_{z}^{j j}$ surge devido ao banho $\sigma_{z}$, que é responsável pela defasagem, com $\gamma_{\phi}(\omega)$ sendo a densidade espectral de ruídos à frequência $\omega$ e $\sigma_{z}^{j k}=\left\langle j\left|\sigma_{z}\right| k\right\rangle$ é o elemento de matriz não-diagonal do termo de defasagem. Neste trabalho não será considerado um modelo específico para as densidades espectrais dos reservatórios. Assim, será usada a hipótese mais simples de que as taxas de dissipação são nulas para $\omega<0$ e constantes para $\omega \geq 0$, sendo idênticas às respectivas taxas na Equação Mestra Padrão.

Devido à dificuldade de se obter soluções analíticas em determinados casos, a Equação Mestra na representação de estados vestidos será resolvida numericamente no capítulo 6, enquanto a Equação Mestra Padrão será utilizada para determinar os principais efeitos dissipativos, tanto analíticamente (no regime assintótico, capítulo 5) quanto numericamente (para tempos finitos, capítulo 6). Por último, ambas as equações mestras serão comparadas com o intuito de se verificar se existem distinções significativas quanto às suas previsões, já que, à primeira vista, a segunda equação é mais completa e deve fornecer resultados mais próximos da realidade. 


\section{Capítulo 2}

\section{Descrição analítica para a EDQ de circuitos não-estacionários}

O problema a ser tratado consiste na interação de $N$ átomos de dois níveis, ou qubits, com um modo do campo eletromagnético confinado em um ressonador guia-de-onda via interação de dipolo. Tal interação átomo-campo é descrita pelo Hamiltoniano generalizado de Rabi [48]:

$$
\hat{H} / \hbar=\omega \hat{n}+\sum_{l=1}^{N}\left[\frac{\Omega}{2} \hat{\sigma}_{z}^{(l)}+g\left(\hat{a}+\hat{a}^{\dagger}\right)\left(\hat{\sigma}_{+}^{(l)}+\hat{\sigma}_{-}^{(l)}\right)+i \chi\left(\hat{a}^{2}+\hat{a}^{\dagger 2}\right)\right],
$$

sendo $\omega$ a frequência da cavidade, $\hat{a}$ and $\hat{a}^{\dagger}$ são os operadores escadas bosônicos e $\hat{n}=\hat{a}^{\dagger} \hat{a}$. Já para o átomo temos os operadores $\hat{\sigma}_{-}^{(l)}=\left|g^{(l)}\right\rangle\left\langle e^{(l)}\left|, \hat{\sigma}_{+}^{(l)}=\right| e^{(l)}\right\rangle\left\langle g^{(l)}\right|$ e $\hat{\sigma}_{z}^{(l)}=\left|e^{(l)}\right\rangle\left\langle e^{(l)}|-| g^{(l)}\right\rangle\left\langle g^{(l)}\right|$,

com $\left|g^{(l)}\right\rangle$ e $\left|e^{(l)}\right\rangle$ representando o estado fundamental e o excitado, respectivamente, para o $l$-ésimo qubit. Supomos modulações temporais da forma $X=X_{0}+\varepsilon_{X} f_{X}$, onde $X=\{\omega, \Omega, g, \chi\}$, tendo: $\omega=\omega_{0}+\varepsilon_{\omega} f_{\omega}$ como a frequência da cavidade, $\Omega=\Omega_{0}+\varepsilon_{\Omega} f_{\Omega}$ como a frequência de transição do átomo, $g=g_{0}+\varepsilon_{g} f_{g}$ o parâmetro de acoplamento átomo-campo e $\chi$ é o coeficiente de squeezing, que é incluso por causa da completeza do Hamiltoniano, embora sua presença não é necessária para implementar os fenômenos considerados aqui. A parte independente do tempo de $\chi$ é oriunda de termos proporcionais ao quadrado do potencial vetorial, que aparecem naturalmente quando se usa o Hamiltoniano de acoplamento mínimo e aproximação de dipolo em primeira ordem ou ordem superior. Usualmente essa parte pode ser eliminada por uma transformação apropriada da função de onda e seu único efeito é alterar ligeiramente as frequências de modulação ressonantes. A parte dependente do tempo é relacionada a alguns processos de amplificação paramétrica [33]. No contexto do regime 
$E C D$ a modulação externa temporal da frequência da cavidade resulta em $\chi=(4 \omega)^{-1} d \omega / d t[32],[33]$. No trabalho, essa parte de $\chi$ não receberá tanta atenção quanto os parâmetros $\omega, \Omega$ e $g$, pois seus efeitos serão qualitativamente similares. A função adimensional que descreve as modulações externas é definida como

$$
f_{X}=\sum_{j} w_{X}^{(j)} \sin \left(\eta^{(j)} t+\phi_{X}^{(j)}\right)=\frac{1}{2 i} \sum_{j} w_{X}^{(j)}\left(e^{i \phi_{X}^{(j)}} e^{i t \eta^{(j)}}-e^{-i \phi_{X}^{(j)}} e^{-i t \eta^{(j)}}\right)
$$

onde $X=\{\omega, g, \Omega, \chi\}, w_{X}^{(j)} \leq 1$ é o peso da modulação $X$ com a $j$-ésima frequência (aqui será considerado $w_{X}^{(j)}=1$ ), $\phi_{X}^{(j)}$ sua fase (aqui no trabalho será considerada igual a zero) e o índice $j$ corre sobre todas as modulações de frequências não nulas $\eta^{(j)}$.

\subsection{Solução Geral}

O Hamiltoniano (2.1) será escrito em termos dos estados normalizados de Dicke, que torna a notação mais simplificada. Para $k$ excitações, onde $k=0,1,2 \ldots, N$, o estado de Dicke é definido como $[42]$ :

$$
|\mathbf{k}\rangle=\sqrt{k !(N-k) ! / N !} \sum_{p}\left|e^{(1)}\right\rangle\left|e^{(2)}\right\rangle \cdots\left|e^{(k)}\right\rangle\left|g^{(k+1)}\right\rangle \cdots\left|g^{N}\right\rangle
$$

onde a soma corre entre todas as possíveis permutações de qubits excitados e não excitados. Assim, o Hamiltoniano (2.1) será reescrito como:

$$
\hat{H} / \hbar=\omega \hat{n}+\sum_{k=1}^{N} E_{k} \hat{\sigma}_{k, k}+\sum_{k=0}^{N-1} G_{k}\left(\hat{a}+\hat{a}^{\dagger}\right)\left(\hat{\sigma}_{k+1, k}+\hat{\sigma}_{k, k+1}\right),
$$

com o operador que descreve o conjunto de estados atômicos $\sigma_{k, j}=|\mathbf{k}\rangle\langle\mathbf{j}|$, com o paramêtro $E_{k}=$ $k \Omega \equiv E_{0, k}+\varepsilon_{E, k} f_{\Omega}$ e $G_{k}=g \sqrt{(k+1)(N-k)} \equiv G_{0, k}+\varepsilon_{G, k} f_{g}$ sendo o acoplamento átomo-campo.

Agora, é proposto um ansatz na representação de Schrödinger, de forma que a função de onda expandida seja:

$$
|\psi(t)\rangle=\sum_{n=0}^{\infty} \sum_{\mathcal{S}} e^{-i t \lambda_{n, \mathcal{S}}} A_{n, \mathcal{S}}(t)\left|\varphi_{n, \mathcal{S}}\right\rangle,
$$

onde $\left|\varphi_{n, \mathcal{S}}\right\rangle$ é o Estado Vestido (dressed state), autoestado com $n$ excitações do Hamiltoniano sem os termos contra-girantes e os dependentes explícitamente do tempo, e $\lambda_{n, \mathcal{S}}$ denotam os respectivos autovalores (ver apêndice D). O índice $\mathcal{S}$ é usado para diferenciar os autoestados com o mesmo 
número de excitações. Já o coeficiente $A_{n, \mathcal{S}}(t)$ é a amplitude de probabilidade para tais estados. Para se obter a solução analítica, a amplitude de probabilidade $A_{n, \mathcal{S}}(t)$ é reescrita em termos de uma nova amplitude $b_{m, \mathcal{T}}(t)$ (que varia lentamente com o tempo) da seguinte forma

$$
\begin{aligned}
A_{m, \mathcal{T}}(t)= & \exp \left[i \sum_{j}^{\prime} \sum_{X=\omega, \Omega, g} \frac{\Gamma_{m, \mathcal{T}, \mathcal{T}}^{X, j}}{\eta^{(j)}}\left[\cos \left(\eta^{(j)} t+\phi_{X}^{(j)}\right)-\cos \phi_{X}^{(j)}\right]\right] \\
& \times\left\{e^{-i t \nu_{m}, \mathcal{T}} b_{m, \mathcal{T}}(t)-\sum_{\mathcal{S} \neq \mathcal{T}} \sum_{j}^{\prime} \sum_{X=\omega, \Omega, g} \frac{\Gamma_{m, \mathcal{T}, \mathcal{T}}^{X, j}}{2 i} e^{-i t \nu_{m, \mathcal{S}}} b_{m, \mathcal{S}}(t)\right. \\
& \times\left[\frac{\left[e^{i t\left(\lambda_{m, \mathcal{T}}-\lambda_{m, \mathcal{S}}+\eta^{(j)}\right)}-1\right]}{\left(\lambda_{m, \mathcal{T}}-\lambda_{m, \mathcal{S}}+\eta^{(j)}\right)} e^{i \phi_{X}^{(j)}}-\frac{\left[e^{i t\left(\lambda_{m, \mathcal{T}}-\lambda_{m, \mathcal{S}}-\eta^{(j)}\right)}-1\right]}{\left(\lambda_{m, \mathcal{S}}-\lambda_{m, \mathcal{T}}-\eta^{(j)}\right)} e^{-i \phi_{X}^{(j)}}\right] .
\end{aligned}
$$

Como mostrado em [42], faz-se necessário eliminar termos que oscilam rapidamente, que são dados de acordo com a frequência de modulação $\eta^{(j)}$, já que eles não produzem acoplamento ressonante entre diferentes amplitudes de probabilidade $b_{m, \mathcal{S}}(t)$. As altas frequências são definidas aqui como $\eta^{(j)} \geq \omega_{0}$, enquanto as baixas frequências $\eta^{(j)}<\omega_{0}$. A equação é escrita sendo denotado o somatório $\sum_{j}^{\prime}$ para todas as altas frequências (posteriormente, o somatório $\sum_{j}^{\prime \prime}$ correrá para todas as baixas frequências) e $\sum_{\mathcal{S}}$ corre para todos os valores permitidos de $\mathcal{S}$ para diferentes autoestados dados por $m$. Os coeficientes são definidos da seguinte forma

$$
\begin{gathered}
\Gamma_{m, \mathcal{T}, \mathcal{S}}^{\omega, j} \equiv \varepsilon_{\omega} w_{\omega}^{(j)}\left\langle\varphi_{m, \mathcal{T}}|\hat{n}| \varphi_{m, \mathcal{S}}\right\rangle \\
\Gamma_{m, \mathcal{T}, \mathcal{S}}^{\Omega, j} \equiv \sum_{k=1}^{N} \varepsilon_{E, k} w_{\Omega}^{(j)}\left\langle\varphi_{m, \mathcal{T}}\left|\hat{\sigma}_{k, k}\right| \varphi_{m, \mathcal{S}}\right\rangle \\
\Gamma_{m, \mathcal{T}, \mathcal{S}}^{g, j} \equiv \sum_{k=0}^{N-1} \varepsilon_{G, k} w_{g}^{(j)}\left\langle\varphi_{m, \mathcal{T}}\left|\left(\hat{a} \hat{\sigma}_{k+1, k}+\hat{a}^{\dagger} \hat{\sigma}_{k, k+1}\right)\right| \varphi_{m, \mathcal{S}}\right\rangle
\end{gathered}
$$

Nota-se que eles são simplesmente os elementos de matriz tomados entre os estados vestidos com o mesmo $m$.

O parâmetro $\nu_{m, \mathcal{T}}$, que independe do tempo, é o shift de frequência e definido como

$$
\nu_{m, \mathcal{T}} \equiv \sum_{\mathcal{S}}\left[\frac{\left|\sum_{k=0}^{N-1} \Lambda_{k, m, \mathcal{S}, \mathcal{T}} G_{0, k}\right|^{2}}{\left(\lambda_{m, \mathcal{T}}-\lambda_{m-2, \mathcal{S}}\right)}-\frac{\left|\sum_{k=0}^{N-1} \Lambda_{k, m+2, \mathcal{T}, \mathcal{S}} G_{0, k}\right|^{2}}{\left(\lambda_{m+2, \mathcal{S}}-\lambda_{m, \mathcal{T}}\right)}\right]+\Delta \nu_{m, \mathcal{T}}
$$

sendo que os seus coeficientes são dados por

$$
\Lambda_{k, m+2, \mathcal{T}, \mathcal{S}}=\left\langle\varphi_{m, \mathcal{T}}\left|\hat{a} \hat{\sigma}_{k, k+1}\right| \varphi_{m+2, \mathcal{S}}\right\rangle
$$


e $\Delta \nu_{m, \mathcal{T}}$ denota pequenas correções para o shift de frequência, que serão desprezados posteriormente. Após cálculos massivos, a equação simplificada para a amplitude de probabilidade $b_{m, \mathcal{T}}(t)$ foi obtida em $[42,43]$

$$
\begin{aligned}
\dot{b}_{m, \mathcal{T}}(t)= & -i \sum_{\mathcal{S}} \sum_{j}^{\prime \prime} \sum_{X=\omega, \Omega, g} e^{i t\left(\tilde{\lambda}_{m, \mathcal{T}}-\tilde{\lambda}_{m, \mathcal{S}}\right)} \Gamma_{m, \mathcal{T}, \mathcal{S}}^{X, j} \sin \left(\eta^{(j)} t+\phi_{X}^{(j)}\right) b_{m, \mathcal{S}}(t) \\
& +\sum_{\mathcal{S}} \sum_{j}^{\prime} \Theta_{m+2, \mathcal{T}, \mathcal{S}} e^{-i t\left(\tilde{\lambda}_{m+2, \mathcal{S}}-\tilde{\lambda}_{m, \mathcal{T}}-\eta^{(j)}\right)} b_{m+2, \mathcal{S}}(t) \\
& -\sum_{\mathcal{S}} \sum_{j}^{\prime} \Theta_{m, \mathcal{S}, \mathcal{T}}^{*} e^{i t\left(\tilde{\lambda}_{m, \mathcal{T}}-\tilde{\lambda}_{m-2, \mathcal{S}}-\eta^{(j)}\right)} b_{m-2, \mathcal{S}}(t),
\end{aligned}
$$

com $\tilde{\lambda}_{m, \mathcal{T}}=\lambda_{m, \mathcal{T}}+\nu_{m, \mathcal{T}}$ sendo os autovalores efetivos e os coeficientes

$$
\begin{aligned}
\Theta_{m+2, \mathcal{T}, \mathcal{S}}= & \frac{1}{2} \sum_{\mathcal{R}} \sum_{X=\omega, \Omega, g}\left[\frac{\sum_{k=0}^{N-1} G_{0, k} \Lambda_{k, m+2, \mathcal{T}, \mathcal{R}}-i \chi_{0} L_{2, m+2, \mathcal{T}, \mathcal{R}}}{\lambda_{m+2, \mathcal{R}}-\lambda_{m+2, \mathcal{S}}+\eta^{(j)}} \Gamma_{m+2, \mathcal{R}, \mathcal{S}}^{X, j} e^{i \phi_{X}^{(j)}}\right. \\
& \left.-\frac{\sum_{k=0}^{N-1} G_{0, k} \Lambda_{k, m+2, \mathcal{R}, \mathcal{S}}-i \chi_{0} L_{2, m+2, \mathcal{R}, \mathcal{S}}}{\lambda_{m, \mathcal{T}}-\lambda_{m, \mathcal{R}}+\eta^{(j)}} \Gamma_{m, \mathcal{T}, \mathcal{R}} e^{i \phi_{X}^{(j)}}\right] \\
& -\frac{1}{2}\left(\sum_{k=0}^{N-1} \varepsilon_{G, k}^{(j)} \Lambda_{k, m+2, \mathcal{T}, \mathcal{S}}-i \varepsilon_{\chi} L_{2, m+2, \mathcal{T}, \mathcal{S}}\right),
\end{aligned}
$$

enquanto as profundidades de modulações são definidas por

$$
\varepsilon_{G, k}^{(j)} \equiv \varepsilon_{G, k} w_{g}^{(j)} e^{i \phi_{g}^{(j)}}, \varepsilon_{X}^{(j)} \equiv \varepsilon_{X} w_{X}^{(j)} e^{i \phi_{X}^{(j)}}(X=\omega, \Omega, g, \chi), G_{k}=G_{0, k}+\varepsilon_{G, k} f_{g} .
$$

Tal resultado foi obtido sob várias aproximações, sendo a mais importante a aproximação de onda girante (Rotating wave approximation (RWA), em inglês).

Segundo [42], as expressões anteriores foram obtidas sob as seguintes condições:

$$
\begin{gathered}
\frac{\left|\sum_{k=0}^{N-1} G_{0, k} \Lambda_{k, m, \mathcal{T}, \mathcal{S}}\right|}{\omega_{0}}, \frac{\left|\chi_{0} L_{2, m+2, \mathcal{T}, \mathcal{S}}\right|}{\omega_{0}}, \frac{\left|\varepsilon_{\chi} L_{2, m+2, \mathcal{T}, \mathcal{S}}\right|}{\omega_{0}} \ll 1 \\
\frac{\varepsilon_{G, k}}{G_{0, k}}, \frac{\left|\lambda_{m, \mathcal{S}}-\lambda_{m, \mathcal{T}}\right|}{\omega_{0}}, \frac{\left|\Gamma_{m+2, \mathcal{S}, \mathcal{S}}^{k, j}-\Gamma_{m, \mathcal{T}, \mathcal{T}}^{k, j}\right|}{\omega_{0}}, \frac{\Gamma_{m, \mathcal{T}, S \neq \mathcal{T}}^{k, j}}{\omega_{0}} \ll 1 .
\end{gathered}
$$

Além disso, as correções para os shifts de frequência são da ordem de

$$
\mathcal{O}\left(\Delta \nu_{m, \mathcal{T}}\right) \simeq \frac{\left|\Gamma_{m, \mathcal{T}, S}^{k, j}\right|^{2}}{\omega_{0}} .
$$

O leitor pode se direcionar para as referências $[42,43,49]$ para maiores detalhes. 


\subsection{Efeitos físicos devidos a modulações ressonantes}

A partir de agora a atenção será restringida ao caso de um único qubit $N=1$. Diante do exposto anteriormente, uma nova função de onda expandida será proposta, que será equivalente à (2.5). Entretanto, a nova função de onda é uma aproximação dado que sua amplitude de probabilidade será $b_{n, \mathcal{S}}(t)$, ou seja, a solução da equação (2.12). Além disso, os autovalores corrigidos $\tilde{\lambda}_{n, \mathcal{S}}=\lambda_{m, \mathcal{T}}+\nu_{m, \mathcal{T}}$, que são os argumentos das exponenciais em (2.12), demonstram a extrema importância dos shifts de ressonância para o ajuste fino da frequência de modulação. A função de onda será, então,

$$
|\psi(t)\rangle \simeq e^{-i t \lambda_{0}} b_{0}(t)\left|\varphi_{0}\right\rangle+\sum_{n=1}^{\infty} \sum_{\mathcal{S}= \pm} e^{-i t \lambda_{n, \mathcal{S}}} b_{n, \mathcal{S}}(t)\left|\varphi_{n, \mathcal{S}}\right\rangle,
$$

$\operatorname{com}\left|\varphi_{n, \mathcal{S}}\right\rangle$ sendo os Estados Vestidos (apêndice D), que são autoestados do Hamiltoniano de JaynesCummings. Enfatizamos aqui que aproximamos $\tilde{\lambda}_{m, \mathcal{S}}$ por $\lambda_{m, \mathcal{S}}$, ou seja, daqui para frente desprezamos nos cálculos analíticos os shifts de frequência de ordem de $g_{0}^{2} / \omega_{0}$. Isto se faz necessário para tratar de modo unificado as dinâmicas unitária e dissipativa (ver capítulo 4).

A partir daqui será também proposto um Hamiltoniano efetivo, que compacta a notação de [43], de modo que será possível obter efeitos relacionados à criação e aniquilação de fótons. Para tanto, tal Hamiltoniano na representação de interação será

$$
\tilde{H}=\sum_{m, \mathcal{S}, \mathcal{T}} \sum_{j} \Xi_{m, \mathcal{T}, \mathcal{S}}^{(j)} e^{-i t\left(\lambda_{m+2, \mathcal{S}}-\lambda_{m, \mathcal{T}}-\eta^{(j)}\right)}\left|\varphi_{m, \mathcal{T}}\right\rangle\left\langle\varphi_{m+2, \mathcal{S}}\right|+h . c .,
$$

onde o termo $\eta^{(j)}$ referente à frequência de modulação será ressonante quando estiver de acordo com a diferença $\lambda_{m+2, \mathcal{S}}-\lambda_{m, \mathcal{T}}$ e, assim, indicará o fenômeno físico a ser tratado. Tais modulações são indicadas em [43] e [49] e serão explicitadas aqui; em seguida será necessário mostrar que o Hamiltoniano (2.17) é uma boa aproximação e a modulação fornece os coeficientes de acoplamento $\Theta_{m+2, \mathcal{T}, \mathcal{S}}$. A afirmação sobre o Hamiltoniano ser uma alternativa mais eficaz para análise do problema deve-se ao fato de que a diferença $\lambda_{m+2, \mathcal{S}}-\lambda_{m, \mathcal{T}}$, quando fora da ressonância, permite que a maioria termos sejam eliminados via RWA, mais especificamente quando $\lambda_{m+2, \mathcal{S}}-\lambda_{m, \mathcal{T}} \gg\left|\Xi_{m, \mathcal{T}, \mathcal{S}}^{(j)}\right|$. Além disso, os shifts de ressonância serão desprezados na correção dos autovalores, eles serão da ordem de $\mathcal{O}\left(g_{0}^{2} / \omega_{0}, \chi_{0}^{2} / \omega_{0}\right)$. Entretanto, isto não traz alterações matemáticas significativas.

Apesar do Hamiltoniano (2.17) ter sido apresentado sem uma justificativa anterior, pode-se mostrar que as aproximações citadas anteriormente fazem com que tal Hamiltoniano forneça equações 
diferenciais para a amplitude de probabilidade que se assemelham à equação diferencial (2.12) (obviamente diferindo por termos desprezados). A equação de Schrödinger fornece para uma função de onda da forma $|\psi(t)\rangle=\sum_{m, \mathcal{T}} c_{m, \mathcal{T}}(t)\left|\varphi_{m, \mathcal{T}}\right\rangle$ a expressão (lembrando que estamos trabalhando na representação de interação)

$$
\begin{aligned}
& i \frac{d}{d t}\left[\sum_{m, \mathcal{T}} c_{m, \mathcal{T}}(t)\left|\varphi_{m, \mathcal{T}}\right\rangle\right]=\tilde{H}\left[\sum_{m, \mathcal{T}} c_{m, \mathcal{T}}(t)\left|\varphi_{m, \mathcal{T}}\right\rangle\right] \\
& =\sum_{m^{\prime}, \mathcal{S}, \mathcal{T}^{\prime}} \sum_{j} \sum_{m, \mathcal{T}} \Xi_{m^{\prime}, \mathcal{T}^{\prime}, \mathcal{S}}^{(j)} e^{-i t\left(\lambda_{\left.m^{\prime}+2, \mathcal{S}^{-}-\lambda_{m^{\prime}, \mathcal{T}^{\prime}}-\eta^{(j)}\right)} c_{m, \mathcal{T}}(t)\left|\varphi_{m^{\prime}, \mathcal{T}^{\prime}}\right\rangle\right.} \\
& \times\left\langle\varphi_{m^{\prime}+2, \mathcal{S}}|| \varphi_{m, \mathcal{T}}\right\rangle \\
& +\sum_{m^{\prime}, \mathcal{S}, \mathcal{T}^{\prime}} \sum_{j} \sum_{m, \mathcal{T}} \Xi_{m^{\prime}, \mathcal{S}, \mathcal{T}^{\prime}}^{*(j)} e^{i t\left(\lambda_{m^{\prime}+2, \mathcal{T}^{\prime}}-\lambda_{m^{\prime}, \mathcal{S}^{-}} \eta^{(j)}\right)} c_{m, \mathcal{T}}(t)\left|\varphi_{m^{\prime}+2, \mathcal{T}^{\prime}}\right\rangle \\
& \times\left\langle\varphi_{m^{\prime}, \mathcal{S}}|| \varphi_{m, \mathcal{T}}\right\rangle
\end{aligned}
$$

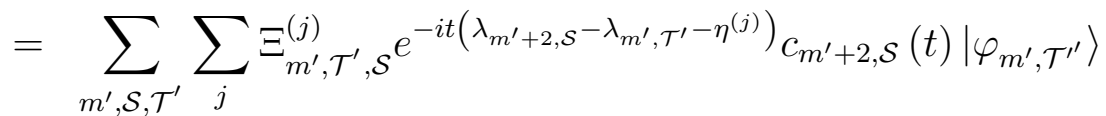

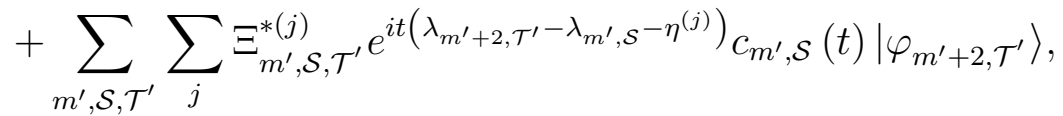

que, quando operamos $\left\langle\varphi_{m, \mathcal{T}}\right|$, tem como resultado final

$$
\begin{aligned}
\dot{c}_{m, \mathcal{T}}(t)= & \sum_{\mathcal{S}} \sum_{j}^{\prime} \Theta_{m+2, \mathcal{T}, \mathcal{S}} e^{-i t\left(\lambda_{m+2, \mathcal{S}}-\lambda_{m, \mathcal{T}}-\eta^{(j)}\right)} c_{m+2, \mathcal{S}}(t) \\
& -\sum_{\mathcal{S}} \sum_{j}^{\prime} \Theta_{m, \mathcal{S}, \mathcal{T}}^{*} e^{i t\left(\lambda_{m, \mathcal{T}}-\lambda_{m-2, \mathcal{S}}-\eta^{(j)}\right)} c_{m-2, \mathcal{S}}(t) .
\end{aligned}
$$

$\mathrm{Na}$ equação anterior, foi usado o coeficiente de acoplamento $\Xi_{m, \mathcal{T}, \mathcal{S}}^{(j)}=i \Theta_{m+2, \mathcal{T}, \mathcal{S}}$, que permitiu tal resultado. O resultado (2.19) será igual à $b_{m, \mathcal{T}}(t)$ ao se eliminar a primeira linha via RWA em (2.12). Daqui em diante, todos os efeitos físicos analisados serão obtidos quando a frequência $\eta^{(j)}$ for ajustada para ressonância, quando será possível obter um Hamiltoniano efetivo simplificado. Tal Hamiltoniano indicará como a dinâmica do sistema se comportará ao tornar explícitos os possíveis acoplamentos entre estados na forma $\left|\varphi_{m, \mathcal{T}}\right\rangle \leftrightarrow\left\langle\varphi_{m+2, \mathcal{S}}\right|$. Assim, embora o Hamiltoniano efetivo não traga novidades em comparação com a equação (2.12), ela permite visualizar o comportamento físico de maneira muito mais intuitiva. Ademais, será mostrado que a expansão em termos de estados vestidos tornará a análise da EMP muito mais simples. 


\section{Capítulo 3}

\section{Equação Mestra na representação dos Estados Vestidos}

Na equação mestra padrão a construção é tomada considerando que o qubit e a cavidade não interagem entre si. Entretanto, quando o acoplamento qubit-cavidade é considerado, uma construção mais sofisticada dos termos dissipativos na EM é necessária. Ao se levar em conta o acoplamento entre os subsistemas, a construção dos termos dissipativos será toda feita sob a representação dos Estados Vestidos. Então, diferentemente da EMP, que foi construída no formalismo de Estados Des-

pidos, o acoplamento $g$ leva a transições entre estados emaranhados qubit-ressonador $\left\{|g, 0\rangle,\left|\varphi_{n, \pm}\right\rangle\right\}$ (EV) induzidas pelos reservatórios. Daqui em diante, por conveniência notacional, os estados serão denotados por $|j\rangle$, com $j$ crescendo de acordo com a energia. Além do mais, o parâmetro de acoplamento $\alpha_{l}$ para sistema-banho, sendo $l$ o modo do banho, será considerado o foco da construção. No texto abaixo $b_{l}$ e $b_{l}^{\dagger}$ são operadores escadas para o modo $l$ do banho e enfatizamos que $\hbar=1$. Este capítulo segue de perto o artigo [16].

\subsection{Formalismo matemático para construção da Equação Mestra}

Inicialmente, considera-se um sistema $S$ amortecido por um reservatório $R$. A discussão a respeito da equação mestra se inicia com o Hamiltoniano da forma 


$$
H=H_{S}+H_{R}+H_{S R}
$$

onde $H_{S}$ e $H_{R}$ são os Hamiltonianos que descrevem o sistema $S$ e o reservatório $R$, respectivamente. Já o termo $H_{S R}$ é o Hamiltoniano para a interação entre $S$ e $R$. Em seguida, considera-se um operador $\chi(t)$ como sendo o operador densidade para $S \oplus R$ e define-se

$$
\rho(t)=\operatorname{Tr}_{R}[\chi(t)]
$$

onde $\rho(t)$ é o operador densidade reduzido quando tomado o traço parcial sobre os estados do reservatório. Para um operador $\hat{O}$, no espaço de Hilbert de $S$, mesmo sem o conhecimento de $\chi(t)$, apenas conhecendo-se o operador densidade $\rho(t)$, é possível calcular seu valor médio na representação de Schrödinger:

$$
\langle\hat{O}\rangle=\operatorname{Tr}_{S \oplus R}[\hat{O} \chi(t)]=\operatorname{Tr}_{S}\left\{\hat{O} \operatorname{Tr}_{R}[\chi(t)]\right\}=\operatorname{Tr}_{S}[\hat{O} \rho(t)] .
$$

Assim, será possível obter uma equação para $\rho(t)$ em que as propriedades de $R$ sejam apenas parâmetros.

A evolução do operador densidade para o sistema $S \oplus R$ é dada por

$$
\dot{\chi}=-i[H, \chi],
$$

com $\hbar=1$ e $H$ dado pela equação (3.1). Primeiro, vamos mudar para a representação de interação fazendo uma transformação unitária com $U=e^{-i\left(H_{S}+H_{R}\right) t}$. Desta forma, reescrevemos

$$
\tilde{\chi}(t)=e^{i\left(H_{S}+H_{R}\right) t} \chi(t) e^{-i\left(H_{S}+H_{R}\right) t},
$$

onde o Hamiltoniano de interação dependente explicitamente do tempo torna-se

$$
\tilde{H}_{S R}(t)=e^{i\left(H_{S}+H_{R}\right) t} H_{S R} e^{-i\left(H_{S}+H_{R}\right) t} .
$$

Daí obtem-se, separando o rápido movimento gerado por $H_{S}$ e $H_{R}$ do movimento lento gerado por $H_{S R}$, a equação diferencial

$$
\frac{d}{d t} \tilde{\chi}=-i\left[\tilde{H}_{S R}(t), \tilde{\chi}\right] .
$$

Integrando formalmente (3.7), o resultado será:

$$
\tilde{\chi}(t)=\chi(0)+\frac{1}{i} \int_{0}^{t} d t^{\prime}\left[\tilde{H}_{S R}\left(t^{\prime}\right), \tilde{\chi}\left(t^{\prime}\right)\right]
$$


e substituindo o comutador em (3.7) para $\tilde{\chi}(t)$ obtém-se exatamente uma equação integro-diferencial

$$
\frac{d}{d t} \tilde{\chi}(t)=\frac{1}{i}\left[\tilde{H}_{S R}(t), \chi(0)\right]-\int_{0}^{t} d t^{\prime}\left[\tilde{H}_{S R}(t),\left[\tilde{H}_{S R}\left(t^{\prime}\right), \tilde{\chi}\left(t^{\prime}\right)\right]\right] .
$$

Será assumido que a interação inicia-se em $t=0$ e que não existem correlações entre $S$ e $R$ no tempo inicial. Assim, $\chi(0)=\tilde{\chi}(0)$ e então

$$
\chi(0)=\rho(0) \rho_{R},
$$

onde $\rho_{R}$ é o operador densidade inicial do reservatório. Note que

$$
\operatorname{Tr}_{R}[\tilde{\chi}]=e^{i H_{S} t} \rho e^{-i H_{S} t}=\tilde{\rho}
$$

fornece

$$
\frac{d}{d t} \tilde{\rho}(t)=-\int_{0}^{t} d t^{\prime} \operatorname{Tr}_{R}\left[\tilde{H}_{S R}(t),\left[\tilde{H}_{S R}\left(t^{\prime}\right), \tilde{\chi}\left(t^{\prime}\right)\right]\right],
$$

onde, por simplicidade, o termo $\frac{1}{i} \operatorname{Tr}_{R}\left\{\left[\tilde{H}_{S R}(t), \chi(0)\right]\right\}$ foi eliminado assumindo que

$\operatorname{Tr}_{R}\left[\tilde{H}_{S R}\left(t^{\prime}\right) \rho_{R}\right]=0$. Isso é garantido se os operadores do reservatório acoplados a $S$ têm valor médio sendo zero no estado $\rho_{R}$ como acontece para reservatórios no equilíbrio térmico.

Logo, em consequência da evolução temporal e assumindo que o acoplamento entre o sistema e o reservatório é fraco, somado ao fato de que o reservatório tem inúmeros graus de liberdade, qualquer variável do reservatório somente pode ser afetada fracamente pelo sistema. Isto permite escrever

$$
\tilde{\chi}(t)=\tilde{\rho}(t) \rho_{R}+O\left(H_{S R}\right) .
$$

Agora, será feita a aproximação de Born, desprezando-se termos superiores à segunda ordem no Hamiltoniano de interação $H_{S R}$

$$
\frac{d}{d t} \tilde{\rho}(t)=-\int_{0}^{t} d t^{\prime} \operatorname{Tr}_{R}\left[\tilde{H}_{S R}(t),\left[\tilde{H}_{S R}\left(t^{\prime}\right), \tilde{\rho}\left(t^{\prime}\right) \rho_{R}\right]\right] .
$$

O comportamento de um sistema Markoviano depende somente do tempo presente. Por enquanto, essa equação não é Markoviana, pois a evolução futura de $\tilde{\rho}(t)$ depende do passado através da integração sobre $\tilde{\rho}\left(t^{\prime}\right)$. Em seguida, outra aproximação será feita, que consiste na troca do operador densidade $\tilde{\rho}\left(t^{\prime}\right)$ ( dependente do tempo passado $t^{\prime}$ ) pelo operador $\tilde{\rho}(t)$ (dependente do tempo presente t). Essa troca é conhecida como aproximação de Markov: 


$$
\frac{d}{d t} \tilde{\rho}(t)=-\int_{0}^{t} d t^{\prime} \operatorname{Tr}_{R}\left[\tilde{H}_{S R}(t),\left[\tilde{H}_{S R}\left(t^{\prime}\right), \tilde{\rho}(t) \rho_{R}\right]\right] .
$$

Agora, o modelo será reescrito de uma forma mais especifica definindo

$$
H_{S R}=\sum_{i} s_{i} B_{i}
$$

onde $s_{i}$ opera no espaço de Hilbert do sistema $S$ e $B_{i}$ opera no espaço de Hilbert do reservatório $R$. Assim, o Hamiltoniano será reescrito na representação de interação como

$$
\tilde{H}_{S R}=\sum_{i} \tilde{s}_{i}(t) \tilde{B}_{i}(t)
$$

que permite reescrever a equação (3.15) da seguinte forma

$$
\begin{aligned}
& \frac{d}{d t} \tilde{\rho}(t)=-\sum_{i, j} \int_{0}^{t} d t^{\prime} \operatorname{Tr}_{R}\left[\tilde{s}_{i}(t) \tilde{B}_{i}(t),\left[\tilde{s}_{j}\left(t^{\prime}\right) \tilde{B}_{j}\left(t^{\prime}\right), \tilde{\rho}\left(t^{\prime}\right) \rho_{R}\right]\right] \\
& =-\sum_{i, j} \int_{0}^{t} d t^{\prime} \operatorname{Tr}_{R}\left\{\left[\tilde{s}_{i}(t) \tilde{B}_{i}(t), \tilde{s}_{j}\left(t^{\prime}\right) \tilde{B}_{j}\left(t^{\prime}\right) \tilde{\rho}\left(t^{\prime}\right) \rho_{R}\right]-\left[\tilde{s}_{i}(t) \tilde{B}_{i}(t), \tilde{\rho}\left(t^{\prime}\right) \rho_{R} \tilde{s}_{j}\left(t^{\prime}\right) \tilde{B}_{j}\left(t^{\prime}\right)\right]\right\} \\
& =-\sum_{i, j} \int_{0}^{t} d t^{\prime} \operatorname{Tr}_{R}\left\{\left[\tilde{s}_{i}(t) \tilde{B}_{i}(t) \tilde{s}_{j}\left(t^{\prime}\right) \tilde{B}_{j}\left(t^{\prime}\right) \tilde{\rho}\left(t^{\prime}\right) \rho_{R}-\tilde{s}_{j}\left(t^{\prime}\right) \tilde{B}_{j}\left(t^{\prime}\right) \tilde{\rho}\left(t^{\prime}\right) \rho_{R} \tilde{s}_{i}(t) \tilde{B}_{i}(t)\right]\right. \\
& \left.-\left[\tilde{s}_{i}(t) \tilde{B}_{i}(t) \tilde{\rho}\left(t^{\prime}\right) \rho_{R} \tilde{s}_{j}\left(t^{\prime}\right) \tilde{B}_{j}\left(t^{\prime}\right)-\tilde{\rho}\left(t^{\prime}\right) \rho_{R} \tilde{s}_{j}\left(t^{\prime}\right) \tilde{B}_{j}\left(t^{\prime}\right) \tilde{s}_{i}(t) \tilde{B}_{i}(t)\right]\right\} \\
& =-\sum_{i, j} \int_{0}^{t} d t^{\prime} \operatorname{Tr}_{R}\left\{\left[\tilde{s}_{i}(t) \tilde{B}_{i}(t) \tilde{s}_{j}\left(t^{\prime}\right) \tilde{B}_{j}\left(t^{\prime}\right) \tilde{\rho}(t) \rho_{R}-\tilde{s}_{j}\left(t^{\prime}\right) \tilde{B}_{j}\left(t^{\prime}\right) \tilde{\rho}\left(t^{\prime}\right) \rho_{R} \tilde{s}_{i}(t) \tilde{B}_{i}(t)\right]\right. \\
& \left.-\left[\tilde{s}_{i}(t) \tilde{B}_{i}(t) \tilde{\rho}\left(t^{\prime}\right) \rho_{R} \tilde{s}_{j}\left(t^{\prime}\right) \tilde{B}_{j}\left(t^{\prime}\right)-\tilde{\rho}\left(t^{\prime}\right) \rho_{R} \tilde{s}_{j}\left(t^{\prime}\right) \tilde{B}_{j}\left(t^{\prime}\right) \tilde{s}_{i}(t) \tilde{B}_{i}(t)\right]\right\} \\
& =-\sum_{i, j} \int_{0}^{t} d t^{\prime}\left\{\left[\tilde{s}_{i}(t) \tilde{s}_{j}\left(t^{\prime}\right) \tilde{\rho}\left(t^{\prime}\right)-\tilde{s}_{j}\left(t^{\prime}\right) \tilde{\rho}\left(t^{\prime}\right) \tilde{s}_{i}(t)\right] \operatorname{Tr}_{R}\left[\rho_{R} \tilde{B}_{i}(t) \tilde{B}_{j}\left(t^{\prime}\right)\right]\right. \\
& \left.-\left[\tilde{s}_{i}(t) \tilde{\rho}\left(t^{\prime}\right) \tilde{s}_{j}\left(t^{\prime}\right)-\tilde{\rho}\left(t^{\prime}\right) \tilde{s}_{j}\left(t^{\prime}\right) \tilde{s}_{i}(t)\right] \operatorname{Tr}_{R}\left[\rho_{R} \tilde{B}_{j}\left(t^{\prime}\right) \tilde{B}_{i}(t)\right]\right\} \\
& =-\sum_{i, j} \int_{0}^{t} d t^{\prime}\left\{\left[\tilde{s}_{i}(t) \tilde{s}_{j}\left(t^{\prime}\right) \tilde{\rho}\left(t^{\prime}\right)-\tilde{s}_{j}\left(t^{\prime}\right) \tilde{\rho}\left(t^{\prime}\right) \tilde{s}_{i}(t)\right]\left\langle\tilde{B}_{i}(t) \tilde{B}_{j}\left(t^{\prime}\right)\right\rangle_{R}\right. \\
& \left.-\left[\tilde{s}_{i}(t) \tilde{\rho}\left(t^{\prime}\right) \tilde{s}_{j}\left(t^{\prime}\right)-\tilde{\rho}\left(t^{\prime}\right) \tilde{s}_{j}\left(t^{\prime}\right) \tilde{s}_{i}(t)\right]\left\langle\tilde{B}_{j}\left(t^{\prime}\right) \tilde{B}_{i}(t)\right\rangle_{R}\right\},
\end{aligned}
$$

onde a propriedade cíclica do traço foi usada e as propriedades do reservatório estão inclusas nas funções de correlação. H. Carmichael [20] justifica que a troca de $\tilde{\rho}\left(t^{\prime}\right)$ por $\tilde{\rho}(t)$ é possível se as funções 
de correlação decaem rapidamente na escala de tempo em que $\tilde{\rho}(t)$ varia. Sob esse argumento, o que se deseja é que as funções de correlação obedeçam a relação

$$
\left\langle\tilde{B}_{i}(t) \tilde{B}_{j}\left(t^{\prime}\right)\right\rangle_{R} \propto \delta\left(t-t^{\prime}\right) .
$$

Assim, a aproximação de Markov depende de duas escalas de tempo amplamente separadas: uma escala de tempo lenta para a dinâmica do sistema $\mathcal{S}$; e uma escala de tempo rápida, caracterizando o decaimento das funções de correlação do reservatório. Isto implica que uma equação mestra Markoviana não permite descrever a dinâmica do sistema em intervalos de tempo muito curtos (em inglês, isso é conhecido como "coarse-grained averaging").

\subsection{Dissipadores para os banhos $\sigma_{x}$ e $X$}

Nesta seção, será deduzido o Liouvilliano referente aos banhos acoplados aos operadores $\sigma_{x}$ e $X$. O reservatório será tomado estando em equilíbrio térmico. Para tal, o ponto de partida será o Hamiltomiano de interação

$$
H_{S B}=\sum_{l} \alpha_{l}\left(c+c^{\dagger}\right)\left(b_{l}+b_{l}^{\dagger}\right)
$$

que engloba o qubit $c \rightarrow \sigma_{-}$ou o ressonador $c \rightarrow a$. Na representação de interação o Hamiltoniano será (inserindo a relação de completeza em termos de estados vestidos)

$$
\begin{aligned}
H_{S B} & =\sum_{l} \alpha_{l} e^{i H_{S} t}\left(c+c^{\dagger}\right) e^{-i H_{S} t}\left(b_{l} e^{-i v_{l} t}+b_{l}^{\dagger} e^{i v_{l} t}\right) \\
& =\sum_{l} \sum_{j} \sum_{k} \alpha_{l} e^{i E_{j} t}|j\rangle\left\langle j\left|\left(c+c^{\dagger}\right)\right| k\right\rangle\langle k| e^{-i E_{k} t}\left(b_{l} e^{-i v_{l} t}+b_{l}^{\dagger} e^{i v_{l} t}\right) \\
& =\sum_{l, j, k} \alpha_{l}|j\rangle\left\langle j\left|\left(c+c^{\dagger}\right)\right| k\right\rangle\langle k| e^{i \Delta_{j k} t}\left(b_{l} e^{-i v_{l} t}+b_{l}^{\dagger} e^{i v_{l} t}\right) \\
& =\sum_{l, j, k} \alpha_{l} C_{j k}|j\rangle\langle k| e^{i \Delta_{j k} t}\left(b_{l} e^{-i v_{l} t}+b_{l}^{\dagger} e^{i v_{l} t}\right),
\end{aligned}
$$

com $C_{j k}=\left\langle j\left|\left(c+c^{\dagger}\right)\right| k\right\rangle$ e $\Delta_{j k}=E_{j}-E_{k}$. Em seguida, o somatório será separado em três partes:

$$
\begin{aligned}
H_{S B}= & \sum_{l, j} \alpha_{l} C_{j j}|j\rangle\left\langle j\left|\left(b_{l} e^{-i v_{l} t}+b_{l}^{\dagger} e^{i v_{l} t}\right)+\sum_{l, k<j} \alpha_{l} C_{j k}\right| j\right\rangle\langle k| e^{i \Delta_{j k} t}\left(b_{l} e^{-i v_{l} t}+b_{l}^{\dagger} e^{i v_{l} t}\right) \\
& +\sum_{l, k>j} \alpha_{l} C_{j k}|j\rangle\langle k| e^{i \Delta_{j k} t}\left(b_{l} e^{-i v_{l} t}+b_{l}^{\dagger} e^{i v_{l} t}\right) .
\end{aligned}
$$


Nos termos onde $j=k$ é possível mostrar que $\left\langle j\left|\left(c+c^{\dagger}\right)\right| j\right\rangle=0$. Entretanto, isso só será possível se os autoestados $|j\rangle$ possuírem uma paridade bem definida, já que $c$ pode ser $\sigma_{-}$referindo-se ao qubit ou a para a cavidade (esses operadores alteram a paridade quando aplicados em um estado). Introduzindo o operador paridade $\Pi=(-1)^{a^{\dagger} a+\sigma_{+} \sigma_{-}}$, pode-se mostrar que $|j\rangle$ tem uma paridade bem definida se $\left[H_{R}, \Pi\right]=0$ (apêndice A). Assim, como o operador $X$ tem paridade ímpar

$$
\langle j|X| j\rangle=\left\langle j\left|\Pi^{-1} \Pi X \Pi^{-1} \Pi\right| j\right\rangle=-\left\langle j\left|\Pi^{-1} X \Pi\right| j\right\rangle=-\langle j|X| j\rangle
$$

esse resultado só será possível se $\langle j|X| j\rangle=0$. Logo, o Hamiltoniano se reduzirá a

$$
H_{S B}=\sum_{l, k<j} \alpha_{l} C_{j k}|j\rangle\left\langle k\left|e^{i \Delta_{j k} t}\left(b_{l} e^{-i v_{l} t}+b_{l}^{\dagger} e^{i v_{l} t}\right)+\sum_{l, k>j} \alpha_{l} C_{j k}\right| j\right\rangle\langle k| e^{i \Delta_{j k} t}\left(b_{l} e^{-i v_{l} t}+b_{l}^{\dagger} e^{i v_{l} t}\right) .
$$

Nos termos onde $k<j$, será trocada a ordem dos índices e usado o fato de que $C_{k j}=C_{j k}^{*}$ e $\Delta_{k j}=-$ $\Delta_{j k}$ devido à condição $k>j$

$$
\begin{aligned}
H_{S B}= & \sum_{l, k>j} \alpha_{l} C_{k j}|k\rangle\left\langle j\left|e^{i \Delta_{k j} t}\left(b_{l} e^{-i v_{l} t}+b_{l}^{\dagger} e^{i v_{l} t}\right)+\sum_{l, k>j} \alpha_{l} C_{j k}\right| j\right\rangle\langle k| e^{i \Delta_{j k} t}\left(b_{l} e^{-i v_{l} t}+b_{l}^{\dagger} e^{i v_{l} t}\right) \\
= & \sum_{l, k>j} \alpha_{l} C_{j k}^{*}|k\rangle\left\langle j\left|e^{-i \Delta_{j k} t}\left(b_{l} e^{-i v_{l} t}+b_{l}^{\dagger} e^{i v_{l} t}\right)+\sum_{l, k>j} \alpha_{l} C_{j k}\right| j\right\rangle\langle k| e^{i \Delta_{j k} t}\left(b_{l} e^{-i v_{l} t}+b_{l}^{\dagger} e^{i v_{l} t}\right) \\
= & \sum_{l, k>j} \alpha_{l} C_{j k}^{*}|k\rangle\langle j|\left(b_{l} e^{-i\left(v_{l}+\Delta_{j k}\right) t}+b_{l}^{\dagger} e^{i\left(v_{l}-\Delta_{j k}\right) t}\right) \\
& +\sum_{l, k>j} \alpha_{l} C_{j k}|j\rangle\langle k| e^{i \Delta_{j k} t}\left(b_{l} e^{-i\left(v_{l}-\Delta_{j k}\right) t}+b_{l}^{\dagger} e^{i\left(v_{l}+\Delta_{j k}\right) t}\right) .
\end{aligned}
$$

Repare que $v_{l}-\Delta_{j k}$ será colocado como $v_{l}+\Delta_{k j}$, de forma que as frequências de transição entre os estados sejam positivas. Diante deste fato, os argumentos das exponenciais que os contêm serão muito maiores que o termo de acoplamento $\alpha_{l} C_{j k}$, permitindo que o método RWA (Apêndice B) seja utilizado para eliminá-los. Os termos restantes de $H_{S B}$ serão reescritos em uma nova notação para que posssamos compactar o Hamiltoniano. Para tanto, definiremos

$$
\tilde{s}(t)=\sum_{j, k>j} C_{j k}|j\rangle\langle k| e^{i \Delta_{j k} t}
$$

e

$$
\tilde{B}(t)=\sum_{l} \alpha_{l} b_{l} e^{-i v_{l} t},
$$

assim, o Hamiltoniano toma a forma

$$
H_{S B}=\tilde{s}(t) \tilde{B}^{\dagger}(t)+\tilde{s}^{\dagger}(t) \tilde{B}(t)
$$


Na equação (3.18), como os índices $i$ e $j$ correm de 1 a 2 , será definido que

$$
\begin{aligned}
s_{1}(t) & =\tilde{s}(t)=\sum_{j, k>j} C_{j k}|j\rangle\langle k| e^{i \Delta_{j k} t}, \\
s_{2}(t) & =\tilde{s}^{\dagger}(t)=\sum_{j, k>j} C_{j k}^{*}|k\rangle\langle j| e^{-i \Delta_{j k} t} \\
B_{1}(t) & =\tilde{B}^{\dagger}(t)=\sum_{l} \alpha_{l}^{*} b_{l}^{\dagger} e^{i v_{l} t} \\
B_{2}(t) & =\tilde{B}(t)=\sum_{l} \alpha_{l} b_{l} e^{-i v_{l} t}
\end{aligned}
$$

que permite escrever a equação mestra como

$$
\begin{aligned}
& \frac{d}{d t} \tilde{\rho}(t)=-\int_{0}^{t} d t^{\prime}\left\{\left[\tilde{s}_{1}(t) \tilde{s}_{1}\left(t^{\prime}\right) \tilde{\rho}\left(t^{\prime}\right)-\tilde{s}_{1}\left(t^{\prime}\right) \tilde{\rho}\left(t^{\prime}\right) \tilde{s}_{1}(t)\right]\left\langle\tilde{B}_{1}(t) \tilde{B}_{1}\left(t^{\prime}\right)\right\rangle_{R}\right. \\
& +\left[\tilde{\rho}\left(t^{\prime}\right) \tilde{s}_{1}\left(t^{\prime}\right) \tilde{s}_{1}(t)-\tilde{s}_{1}(t) \tilde{\rho}\left(t^{\prime}\right) \tilde{s}_{1}\left(t^{\prime}\right)\right]\left\langle\tilde{B}_{1}\left(t^{\prime}\right) \tilde{B}_{1}(t)\right\rangle_{R} \\
& +\left[\tilde{s}_{1}(t) \tilde{s}_{2}\left(t^{\prime}\right) \tilde{\rho}\left(t^{\prime}\right)-\tilde{s}_{2}\left(t^{\prime}\right) \tilde{\rho}\left(t^{\prime}\right) \tilde{s}_{1}(t)\right]\left\langle\tilde{B}_{1}(t) \tilde{B}_{2}\left(t^{\prime}\right)\right\rangle_{R} \\
& +\left[\tilde{\rho}\left(t^{\prime}\right) \tilde{s}_{2}\left(t^{\prime}\right) \tilde{s}_{1}(t)-\tilde{s}_{1}(t) \tilde{\rho}\left(t^{\prime}\right) \tilde{s}_{2}\left(t^{\prime}\right)\right]\left\langle\tilde{B}_{2}\left(t^{\prime}\right) \tilde{B}_{1}(t)\right\rangle_{R} \\
& +\left[\tilde{s}_{2}(t) \tilde{s}_{1}\left(t^{\prime}\right) \tilde{\rho}\left(t^{\prime}\right)-\tilde{s}_{1}\left(t^{\prime}\right) \tilde{\rho}\left(t^{\prime}\right) \tilde{s}_{2}(t)\right]\left\langle\tilde{B}_{2}(t) \tilde{B}_{1}\left(t^{\prime}\right)\right\rangle_{R} \\
& +\left[\tilde{\rho}\left(t^{\prime}\right) \tilde{s}_{1}\left(t^{\prime}\right) \tilde{s}_{2}(t)-\tilde{s}_{2}(t) \tilde{\rho}\left(t^{\prime}\right) \tilde{s}_{1}\left(t^{\prime}\right)\right]\left\langle\tilde{B}_{1}\left(t^{\prime}\right) \tilde{B}_{2}(t)\right\rangle_{R} \\
& +\left[\tilde{s}_{2}(t) \tilde{s}_{2}\left(t^{\prime}\right) \tilde{\rho}\left(t^{\prime}\right)-\tilde{s}_{2}\left(t^{\prime}\right) \tilde{\rho}\left(t^{\prime}\right) \tilde{s}_{2}(t)\right]\left\langle\tilde{B}_{2}(t) \tilde{B}_{2}\left(t^{\prime}\right)\right\rangle_{R} \\
& \left.+\left[\tilde{\rho}\left(t^{\prime}\right) \tilde{s}_{2}\left(t^{\prime}\right) \tilde{s}_{2}(t)-\tilde{s}_{2}(t) \tilde{\rho}\left(t^{\prime}\right) \tilde{s}_{2}\left(t^{\prime}\right)\right]\left\langle\tilde{B}_{2}\left(t^{\prime}\right) \tilde{B}_{2}(t)\right\rangle_{R}\right\} \\
& =-\int_{0}^{t} d t^{\prime}\left\{\left[\tilde{s}(t) \tilde{s}\left(t^{\prime}\right) \tilde{\rho}\left(t^{\prime}\right)-\tilde{s}\left(t^{\prime}\right) \tilde{\rho}\left(t^{\prime}\right) \tilde{s}(t)\right]\left\langle\tilde{B}^{\dagger}(t) \tilde{B}^{\dagger}\left(t^{\prime}\right)\right\rangle_{R}\right. \\
& +\left[\tilde{\rho}\left(t^{\prime}\right) \tilde{s}\left(t^{\prime}\right) \tilde{s}(t)-\tilde{s}(t) \tilde{\rho}\left(t^{\prime}\right) \tilde{s}\left(t^{\prime}\right)\right]\left\langle\tilde{B}^{\dagger}\left(t^{\prime}\right) \tilde{B}^{\dagger}(t)\right\rangle_{R} \\
& +\left[\tilde{s}(t) \tilde{s}^{\dagger}\left(t^{\prime}\right) \tilde{\rho}\left(t^{\prime}\right)-\tilde{s}^{\dagger}\left(t^{\prime}\right) \tilde{\rho}\left(t^{\prime}\right) \tilde{s}(t)\right]\left\langle\tilde{B}^{\dagger}(t) \tilde{B}\left(t^{\prime}\right)\right\rangle_{R} \\
& +\left[\tilde{\rho}\left(t^{\prime}\right) \tilde{s}^{\dagger}\left(t^{\prime}\right) \tilde{s}(t)-\tilde{s}(t) \tilde{\rho}\left(t^{\prime}\right) \tilde{s}^{\dagger}\left(t^{\prime}\right)\right]\left\langle\tilde{B}\left(t^{\prime}\right) \tilde{B}^{\dagger}(t)\right\rangle_{R} \\
& +\left[\tilde{s}^{\dagger}(t) \tilde{s}\left(t^{\prime}\right) \tilde{\rho}\left(t^{\prime}\right)-\tilde{s}\left(t^{\prime}\right) \tilde{\rho}\left(t^{\prime}\right) \tilde{s}^{\dagger}(t)\right]\left\langle\tilde{B}(t) \tilde{B}^{\dagger}\left(t^{\prime}\right)\right\rangle_{R} \\
& +\left[\tilde{\rho}\left(t^{\prime}\right) \tilde{s}\left(t^{\prime}\right) \tilde{s}^{\dagger}(t)-\tilde{s}^{\dagger}(t) \tilde{\rho}\left(t^{\prime}\right) \tilde{s}\left(t^{\prime}\right)\right]\left\langle\tilde{B}^{\dagger}\left(t^{\prime}\right) \tilde{B}(t)\right\rangle_{R} \\
& +\left[\tilde{s}^{\dagger}(t) \tilde{s}^{\dagger}\left(t^{\prime}\right) \tilde{\rho}\left(t^{\prime}\right)-\tilde{s}^{\dagger}\left(t^{\prime}\right) \tilde{\rho}\left(t^{\prime}\right) \tilde{s}^{\dagger}(t)\right]\left\langle\tilde{B}(t) \tilde{B}\left(t^{\prime}\right)\right\rangle_{R} \\
& \left.+\left[\tilde{\rho}\left(t^{\prime}\right) \tilde{s}^{\dagger}\left(t^{\prime}\right) \tilde{s}^{\dagger}(t)-\tilde{s}^{\dagger}(t) \tilde{\rho}\left(t^{\prime}\right) \tilde{s}^{\dagger}\left(t^{\prime}\right)\right]\left\langle\tilde{B}\left(t^{\prime}\right) \tilde{B}(t)\right\rangle_{R}\right\} .
\end{aligned}
$$


As funções de correlação serão explicitamente calculadas:

$$
\begin{aligned}
\left\langle\tilde{B}^{\dagger}(t) \tilde{B}^{\dagger}\left(t^{\prime}\right)\right\rangle & =\left\langle\sum_{l} \alpha_{l}^{*} b_{l}^{\dagger} e^{i v_{l} t} \alpha_{l}^{*} b_{l}^{\dagger} e^{i v_{l} t^{\prime}}\right\rangle_{R}=\sum_{l} \alpha_{l}^{*} \alpha_{l}^{*} e^{i v_{l}\left(t+t^{\prime}\right)}\left\langle b_{l}^{\dagger} b_{l}^{\dagger}\right\rangle_{R}=0, \\
\left\langle\tilde{B}^{\dagger}\left(t^{\prime}\right) \tilde{B}^{\dagger}(t)\right\rangle & =\sum_{l} \alpha_{l}^{*} \alpha_{l}^{*} e^{i v_{l}\left(t+t^{\prime}\right)}\left\langle b_{l}^{\dagger} b_{l}^{\dagger}\right\rangle_{R}=0 \\
\left\langle\tilde{B}(t) \tilde{B}\left(t^{\prime}\right)\right\rangle & =\left\langle\sum_{l} \alpha_{l} b_{l} e^{-i v_{l} t} \alpha_{l} b_{l} e^{-i v_{l} t^{\prime}}\right\rangle_{R}=\sum_{l} \alpha_{l} \alpha_{l} e^{-i v_{l}\left(t+t^{\prime}\right)}\left\langle b_{l} b_{l}\right\rangle_{R}=0 \\
\left\langle\tilde{B}\left(t^{\prime}\right) \tilde{B}(t)\right\rangle & =\sum_{l} \alpha_{l} \alpha_{l} e^{-i v_{l}\left(t+t^{\prime}\right)}\left\langle b_{l} b_{l}\right\rangle=0, \\
\left\langle\tilde{B}^{\dagger}(t) \tilde{B}\left(t^{\prime}\right)\right\rangle & =\left\langle\sum_{l} \alpha_{l}^{*} b_{l}^{\dagger} e^{i v_{l} t} \alpha_{l} b_{l} e^{-i v_{l} t^{\prime}}\right\rangle_{R}=\sum_{l} \alpha_{l}^{*} \alpha_{l} e^{i v_{l}\left(t-t^{\prime}\right)}\left\langle b_{l}^{\dagger} b_{l}\right\rangle_{R} \\
& =\sum_{l} \alpha_{l}^{*} \alpha_{l} e^{i v_{l}\left(t-t^{\prime}\right)} \bar{n}(v, T), \\
\left\langle\tilde{B}^{\dagger}\left(t^{\prime}\right) \tilde{B}(t)\right\rangle & =\left\langle\sum_{l} \alpha_{l}^{*} b_{l}^{\dagger} e^{i v_{l} t^{\prime}} \alpha_{l} b_{l} e^{-i v_{l} t}\right\rangle_{R}=\sum_{l} \alpha_{l}^{*} \alpha_{l} e^{-i v_{l}\left(t-t^{\prime}\right)} \bar{n}(v, T), \\
\left\langle\tilde{B}(t) \tilde{B}^{\dagger}\left(t^{\prime}\right)\right\rangle & =\left\langle\sum_{l} \alpha_{l} b_{l} e^{-i v_{l} t} \alpha_{l}^{*} b_{l}^{\dagger} e^{i v_{l} t^{\prime}}\right\rangle_{R}=\sum_{l} \alpha_{l} \alpha_{l}^{*} e^{-i v_{l}\left(t-t^{\prime}\right)}\left\langle b_{l} b_{l}^{\dagger}\right\rangle_{R} \\
& =\sum_{l} \alpha_{l} \alpha_{l}^{*} e^{-i v_{l}\left(t-t^{\prime}\right)}\left\langle b_{l}^{\dagger} b_{l}+1\right\rangle_{R}=\sum_{l} \alpha_{l} \alpha_{l}^{*} e^{-i v_{l}\left(t-t^{\prime}\right)}[\bar{n}(v, T)+1], \\
\left\langle\tilde{B}\left(t^{\prime}\right) \tilde{B}^{\dagger}(t)\right\rangle & =\left\langle\sum_{l} \alpha_{l} b_{l} e^{-i v_{l} t^{\prime}} \alpha_{l}^{*} b_{l}^{\dagger} e^{i v_{l} t}\right\rangle_{R}=\sum_{l} \alpha_{l} \alpha_{l}^{*} e^{i v_{l}\left(t-t^{\prime}\right)}[\bar{n}(v, T)+1]
\end{aligned}
$$

$\operatorname{com} \bar{n}(v, T)=\operatorname{Tr}_{R}\left(\rho_{R} b_{l}^{\dagger} b_{l}\right)$ sendo o número médio de fótons para um oscilador com frequência $v$ em equilíbro térmico à temperatura $T$. Note que $\bar{n}(v, T)$ foi obtido através da propriedade cíclica do traço.

Eliminando as funções de correlação nulas da EM e fazendo uma troca de variáveis $\tau=t-t^{\prime}$, 
chegamos a

$$
\begin{aligned}
\frac{d}{d t} \tilde{\rho}(t)= & \int_{0}^{t} d \tau\left\{\left[\sum_{j, k>j}\left|C_{j k}\right|^{2} e^{i \Delta_{j k} t}|k\rangle\langle j|\tilde{\rho}(t-\tau)| j\rangle\left\langle\left. k\left|-\sum_{j, k>j}\right| C_{j k}\right|^{2} e^{i \Delta_{j k} t} \mid j\right\rangle\langle k|| k\rangle\langle j| \tilde{\rho}(t-\tau)\right]\right. \\
& \times\left\langle\tilde{B}^{\dagger}(t) \tilde{B}(t-\tau)\right\rangle_{R} \\
& +\left[\sum_{j, k>j}\left|C_{j k}\right|^{2} e^{i \Delta_{j k} t}|j\rangle\langle k|\tilde{\rho}(t-\tau)| k\rangle\left\langle\left. j\left|-\sum_{j, k>j}\right| C_{j k}\right|^{2} e^{i \Delta_{j k} t} \tilde{\rho}(t-\tau) \mid k\right\rangle\langle j|| j\rangle\langle k|\right] \\
& \times\left\langle\tilde{B}(t-\tau) \tilde{B}^{\dagger}(t)\right\rangle_{R} \\
& +\left[\sum_{j, k>j}\left|C_{j k}\right|^{2} e^{-i \Delta_{j k} t}|j\rangle\langle k|\tilde{\rho}(t-\tau)| k\rangle\left\langle\left. j\left|-\sum_{j, k>j}\right| C_{j k}\right|^{2} e^{-i \Delta_{j k} t} \mid k\right\rangle\langle j|| j\rangle\langle k| \tilde{\rho}(t-\tau)\right] \\
& \times\left\langle\tilde{B}(t) \tilde{B}^{\dagger}(t-\tau)\right\rangle_{R} \\
& +\left[\sum _ { j , k > j } | C _ { j k } | ^ { 2 } e ^ { - i \Delta _ { j k } t } | k \rangle \langle j | \tilde { \rho } ( t - \tau ) | j \rangle \left\langle\left.k\left|-\sum_{j, k>j}\right| C_{j k}\right|^{2} e^{\left.-i \Delta_{j k} t \tilde{\rho}(t-\tau)|j\rangle\langle k|| k\rangle\langle j|\right]}\right.\right. \\
& \left.\times\left\langle\tilde{B}^{\dagger}(t-\tau) \tilde{B}(t)\right\rangle_{R}\right\} .
\end{aligned}
$$

Introduzindo a densidade de estados $D(v)$, tal que $D(v) d v$ fornece o número de osciladores com frequência no intervalo $v$ a $v+d v$, nós poderemos trocar o somatório por uma integral [20], obtendo:

$$
\begin{aligned}
\left\langle\tilde{B}^{\dagger}(t) \tilde{B}(t-\tau)\right\rangle_{R} & =\sum_{l}\left|\alpha_{l}\right|^{2} e^{i v_{l} \tau} \bar{n}(v, T) \rightarrow \int_{0}^{\infty} d v e^{i v_{l} \tau} D(v)\left|\alpha_{l}(\nu)\right|^{2} \bar{n}(v, T) ; \\
\left\langle\tilde{B}^{\dagger}(t-\tau) \tilde{B}(t)\right\rangle_{R} & =\sum_{l}\left|\alpha_{l}\right|^{2} e^{-i v_{l} \tau} \bar{n}(v, T) \rightarrow \int_{0}^{\infty} d v e^{i v_{l} \tau} D(v)\left|\alpha_{l}(v)\right|^{2} \bar{n}(v, T) ; \\
\left\langle\tilde{B}(t) \tilde{B}^{\dagger}(t-\tau)\right\rangle_{R} & =\sum_{l}\left|\alpha_{l}\right|^{2} e^{-i v_{l} \tau}[\bar{n}(v, T)+1] \\
& \rightarrow \int_{0}^{\infty} d v e^{-i v_{l} \tau} D(v)\left|\alpha_{l}(v)\right|^{2}[\bar{n}(v, T)+1] \\
\left\langle\tilde{B}(t-\tau) \tilde{B}^{\dagger}(t)\right\rangle_{R} & =\sum_{l}\left|\alpha_{l}\right|^{2} e^{i v_{l} \tau}[\bar{n}(v, T)+1] \\
& \rightarrow \int_{0}^{\infty} d v e^{-i v_{l} \tau} D(v)\left|\alpha_{l}(v)\right|^{2}[\bar{n}(v, T)+1] .
\end{aligned}
$$


Logo, a EM toma a forma

$$
\begin{aligned}
\frac{d}{d t} \tilde{\rho}(t)= & \int_{0}^{t} d \tau \int_{0}^{\infty} d v \sum_{j, k>j}\left|C_{j k}\right|^{2} D(v)\left|\alpha_{l}(v)\right|^{2} \\
& \times\left\{e^{i v_{l} \tau} e^{i \Delta_{j k} t} \bar{n}(v, T)[|k\rangle\langle j|\tilde{\rho}(t-\tau)| j\rangle\langle k|-| j\rangle\langle j| \tilde{\rho}(t-\tau)]\right. \\
& +e^{-i v_{l} \tau} e^{i \Delta_{j k} t}[\bar{n}(v, T)+1][|j\rangle\langle k|\tilde{\rho}(t-\tau)| k\rangle\langle j|-\tilde{\rho}(t-\tau)| k\rangle\langle k|] \\
& +e^{-i v_{l} \tau} e^{-i \Delta_{j k} t}[\bar{n}(v, T)+1][|j\rangle\langle k|\tilde{\rho}(t-\tau)| k\rangle\langle j|-| k\rangle\langle k| \tilde{\rho}(t-\tau)] \\
& \left.+e^{i v_{l} \tau} e^{-i \Delta_{j k} t} \bar{n}(v, T)[|k\rangle\langle j|\tilde{\rho}(t-\tau)| j\rangle\langle k|-\tilde{\rho}(t-\tau)| j\rangle\langle j|]\right\} \\
= & \int_{0}^{\infty} d \tau \int_{0}^{\infty} d v \sum_{j, k>j}\left|C_{j k}\right|^{2} D(v)\left|\alpha_{l}(v)\right|^{2}\left\{e^{i\left(v_{l}+\Delta_{j k}\right) t}[|j\rangle\langle k|\tilde{\rho}(t-\tau)| k\rangle\langle j|-\tilde{\rho}(t-\tau)| k\rangle\langle k|]\right. \\
& +e^{-i\left(v_{l}+\Delta_{j k}\right) t}[|j\rangle\langle k|\tilde{\rho}(t-\tau)| k\rangle\langle j|-| k\rangle\langle k| \tilde{\rho}(t-\tau)] \\
& +e^{i\left(v_{l}+\Delta_{j k}\right) t} \bar{n}(\nu, T)[|k\rangle\langle j|\tilde{\rho}(t-\tau)| j\rangle\langle k|-| j\rangle\langle j| \tilde{\rho}(t-\tau) \\
& +|j\rangle\langle k|\tilde{\rho}(t-\tau)| k\rangle\langle j|-\tilde{\rho}(t-\tau)| k\rangle\langle k|] \\
& +e^{-i\left(v_{l}+\Delta_{j k}\right) t} \bar{n}(\nu, T)[|j\rangle\langle k|\tilde{\rho}(t-\tau)| k\rangle\langle j|-| k\rangle\langle k| \tilde{\rho}(t-\tau) \\
& +|k\rangle\langle j|\tilde{\rho}(t-\tau)| j\rangle\langle k|-\tilde{\rho}(t-\tau)| j\rangle\langle j|]\} .
\end{aligned}
$$

Os tempos $\tau$ que dominam a integral são muito pequenos em comparação com a escala de tempo que controla a evolução de $\tilde{\rho}$, portanto será feita a troca de $\tilde{\rho}(t-\tau)$ por $\tilde{\rho}(t)$. Além do mais, esse fato permite estender o tempo $t$ até o infinito, já que a integração em $\tau$ é dominada por tempos muito menores que a escala de tempo $t$. Logo

$$
\begin{aligned}
\lim _{t \rightarrow \infty} \int_{0}^{t} d \tau e^{-i\left(v_{l}+\Delta_{j k}\right) t} & =\pi \delta\left(v_{l}+\Delta_{j k}\right)-i \frac{\mathcal{P}}{v_{l}+\Delta_{j k}}, \\
\lim _{t \rightarrow \infty} \int_{0}^{t} d \tau e^{i\left(v_{l}+\Delta_{j k}\right) t} & =\lim _{t \rightarrow \infty} \int_{0}^{t} d \tau e^{-i\left(-v_{l}-\Delta_{j k}\right) t}=\pi \delta\left(-v_{l}-\Delta_{j k}\right)+i \frac{\mathcal{P}}{v_{l}+\Delta_{j k}} \\
& =\pi \delta\left[-\left(v_{l}+\Delta_{j k}\right)\right]+i \frac{\mathcal{P}}{v_{l}+\Delta_{j k}}=\pi \delta\left(v_{l}+\Delta_{j k}\right)+i \frac{\mathcal{P}}{v_{l}+\Delta_{j k}},
\end{aligned}
$$


onde $\mathcal{P}$ é o valor principal de Cauchy. Após fazermos $\Delta_{j k}=-\Delta_{k j}$

$$
\begin{aligned}
\frac{d}{d t} \tilde{\rho}(t)= & \sum_{j, k>j} \pi d\left(\Delta_{k j}\right) \alpha^{2}\left(\Delta_{k j}\right)\left|C_{j k}\right|^{2} \\
& \times[|j\rangle\langle k|\tilde{\rho}(t)| k\rangle\langle j|+| j\rangle\langle k|\tilde{\rho}(t)| k\rangle\langle j|-\tilde{\rho}(t)| k\rangle\langle k|-| k\rangle\langle k| \tilde{\rho}(t)] \\
& +\sum_{j, k>j}\left|C_{j k}\right|^{2} i \mathcal{P} \int_{0}^{\infty} d \nu \frac{d(\nu) \alpha^{2}(\nu)}{v_{l}+\Delta_{j k}} \\
& \times[|j\rangle\langle k|\tilde{\rho}(t)| k\rangle\langle j|-\tilde{\rho}(t)| k\rangle\langle k|+| k\rangle\langle k|\tilde{\rho}(t)-| j\rangle\langle k|\tilde{\rho}(t)| k\rangle\langle j|] \\
& +\sum_{j, k>j} \pi d\left(\Delta_{k j}\right) \alpha^{2}\left(\Delta_{k j}\right)\left|C_{j k}\right|^{2} \bar{n}\left(\Delta_{j k}, T\right) \\
& \times\{[|k\rangle\langle j|\tilde{\rho}(t)| j\rangle\langle k|-| j\rangle\langle j|\tilde{\rho}(t)+| j\rangle\langle k|\tilde{\rho}(t)| k\rangle\langle j|-\tilde{\rho}(t)| k\rangle\langle k|] \\
& +[|j\rangle\langle k|\tilde{\rho}(t)| k\rangle\langle j|-| k\rangle\langle k|\tilde{\rho}(t)+| k\rangle\langle j|\tilde{\rho}(t)| j\rangle\langle k|-\tilde{\rho}(t)| j\rangle\langle j|]\} \\
& +\sum_{j, k>j}\left|C_{j k}\right|^{2} \bar{n}\left(\Delta_{j k}, T\right) i \mathcal{P} \int_{0}^{\infty} d \nu \frac{d(\nu) \alpha^{2}(\nu)}{v_{l}+\Delta_{j k}} \\
& \times\{[|k\rangle\langle j|\tilde{\rho}(t)| j\rangle\langle k|-| j\rangle\langle j|\tilde{\rho}(t)+| j\rangle\langle k|\tilde{\rho}(t)| k\rangle\langle j|-\tilde{\rho}(t)| k\rangle\langle k|] \\
& -[|j\rangle\langle k|\tilde{\rho}(t)| k\rangle\langle j|-| k\rangle\langle k|\tilde{\rho}(t)+| k\rangle\langle j|\tilde{\rho}(t)| j\rangle\langle k|-\tilde{\rho}(t)| j\rangle\langle j|]\} .
\end{aligned}
$$

Para voltar à representação de Schrödinger, basta fazer outra transformação

$$
\dot{\rho}(t)=-i\left[H_{s}, \rho\right]+e^{-i H_{S} t}\left[\frac{d}{d t} \tilde{\rho}(t)\right] e^{i H_{S} t} .
$$

Logo, podemos organizar os termos de forma que se chegue ao Hamiltoniano

$$
H_{S}^{\prime}(t)=H_{S}-\sum_{j k}\left|C_{j k}\right|^{2}\left\{L_{j k}|k\rangle\langle k|+\left[L_{j k}^{\prime}(|k\rangle\langle k|+| j\rangle\langle j|)\right]\right\},
$$

com os coeficientes dados por

$$
\begin{aligned}
L_{j k} & =\frac{\mathcal{P}}{2 \pi} \int_{0}^{\infty} d v \frac{\Gamma(\nu)}{v_{l}+\Delta_{j k}} ; \\
L_{j k}^{\prime} & =\frac{\mathcal{P}}{2 \pi} \int_{0}^{\infty} d v \frac{\Gamma(\nu)}{v_{l}+\Delta_{j k}} \bar{n}\left(\Delta_{k j}, T\right) ; \\
\Gamma^{j k} & =2 \pi D\left(\Delta_{k j}\right) \alpha^{2}\left(\Delta_{k j}\right)\left|C_{j k}\right|^{2} .
\end{aligned}
$$


O resultado final será

$$
\begin{aligned}
\dot{\rho}(t)= & -i\left[H_{S}^{\prime}, \rho(t)\right]+\sum_{j, k>j} \Gamma^{j k}\left[|j\rangle\langle k|\rho(t)| k\rangle\left\langle j\left|-\frac{1}{2} \rho(t)\right| k\right\rangle\left\langle k\left|-\frac{1}{2}\right| k\right\rangle\langle k| \rho(t)\right] \\
& +\sum_{j, k>j} \Gamma^{j k} \bar{n}\left(\Delta_{k j}, T\right)\left[|j\rangle\langle k|\rho(t)| k\rangle\left\langle j\left|-\frac{1}{2} \rho(t)\right| k\right\rangle\left\langle k\left|-\frac{1}{2}\right| k\right\rangle\langle k| \rho(t)\right] \\
& +\sum_{j, k>j} \Gamma^{j k} \bar{n}\left(\Delta_{k j}, T\right)\left[|k\rangle\langle j|\rho(t)| j\rangle\left\langle k\left|-\frac{1}{2} \rho(t)\right| j\right\rangle\left\langle j\left|-\frac{1}{2}\right| j\right\rangle\langle j| \rho(t)\right] \\
= & -i\left[H_{S}^{\prime}, \rho(t)\right]+\sum_{j, k>j} \Gamma^{j k} \bar{n}\left(\Delta_{k j}, T\right) \hat{\mathcal{D}}[|k\rangle\langle j|] \rho(t)+\sum_{j, k>j} \Gamma^{j k}\left[1+\bar{n}\left(\Delta_{k j}, T\right)\right] \hat{\mathcal{D}}[|j\rangle\langle k|] \rho(t) .
\end{aligned}
$$

O Hamiltoniano $H_{S}^{\prime}(t)$ é o Hamiltoniano Lamb-shifted do sistema e $L_{j k}^{\prime}$ são os Lamb shifts causados pelo acoplamento ao ambiente. A função $\Gamma(\nu)$ é a taxa de relaxação. No caso da perda de fótons, a troca $c \rightarrow a$ será feita e a taxa de relaxação $\Gamma(\nu)$ será trocada por $\kappa(\nu)$. No caso da relaxação atômica, a troca entre os operadores $c \rightarrow \sigma_{-}$será feita e a taxa $\Gamma(\nu)$ será trocada por $\gamma(\nu)$. Além disso, os Lamb shifts serão desprezados.

\subsection{Dissipadores para o banho $\sigma_{z}$}

\subsubsection{Modelo clássico}

Agora, será demonstrada a parte da defasagem da equação (1.3). Para isso, define-se a função $f(t)$ em

$$
H_{d e f}=f(t) \sigma_{z}
$$

como uma função estocástica, que modula a frequência do qubit, com valor médio nulo. Em seguida, expressa-se o Hamiltoniano em termos dos estados vestidos para, em seguida, ir à representação de interação:

$$
H_{\text {def }}(t)=\sum_{j k} f(t)|j\rangle\left\langle k\left|\left\langle j\left|\sigma_{z}\right| k\right\rangle e^{i \Delta_{j k} t}=\sum_{j k} f(t)\right| j\right\rangle\langle k| \sigma_{z}^{j k} e^{i \Delta_{j k} t},
$$

onde $f(t)$ será expressado em termos da Transformada de Fourier (apêndice C). Para tal, algumas definições que garantam essa possibilidade são necessárias.

Então, $f(t)$ será definido como

$$
f(t)=\int_{-\infty}^{\infty} d \omega f(\omega) e^{i \omega t}
$$


de forma que se possa escrever

$$
H_{d e f}(t)=\sum_{j k}|j\rangle\langle k| \sigma_{z}^{j k} f_{-\Delta_{j k}}(t)
$$

sendo

$$
f_{\Delta_{j k}}(t)=\int_{\Delta_{j k}-\vartheta_{j k}}^{\Delta_{j k}+\vartheta_{j k}} d \omega f(\omega) e^{i\left(\omega-\Delta_{j k}\right) t} .
$$

Nesse intervalo de frequência $2 \vartheta_{j k}$ em torno de $\Delta_{j k}$ considera-se que vem a maior contribuição para a defasagem, de forma que a aproximação é válida se $\vartheta_{j k} \ll \Delta_{j k}$. Com o auxílio do Teorema de Wiener-Khintchin, tem-se

$$
E\left[f(\omega) f\left(-\omega^{\prime}\right)\right]=\delta\left(\omega-\omega^{\prime}\right) S_{f}(\omega)
$$

onde $E[x]$ é o valor médio clássico de $x$ e $S_{f}(\omega)$ é a densidade espectral de $f(t)$. Isso permite escrever, então,

$$
f(\omega)=\sqrt{S_{f}(\omega)} \xi(\omega)
$$

de modo que o valor médio clássico da função $\xi(\omega)$ seja nulo $E[\xi(\omega)]=0$ e $E\left[\xi(\omega) \xi\left(-\omega^{\prime}\right)\right]=$ $\delta\left(\omega-\omega^{\prime}\right)$. Assim, com $S_{f}(\omega)$ sendo aproximadamente constante em cada componente individual $B_{j k}$ :

$$
f_{\Delta_{j k}}(t)=\sqrt{S_{f}\left(\Delta_{j k}\right)} \int_{-\vartheta_{j k}}^{\vartheta_{j k}} d \omega \xi\left(\omega+\Delta_{j k}\right) e^{i \omega t} .
$$

Ao definir $\vartheta_{j k}$ um intervalo muito pequeno de frequência, a escala de tempo de defasagem será muito mais lenta que $1 / \vartheta_{j k}$, permitindo fazer formalmente $\vartheta_{j k} \rightarrow \infty$. Logo,

$$
f_{\Delta_{j k}}(t)=\sqrt{S_{f}\left(\Delta_{j k}\right)} \int_{-\infty}^{\infty} d \omega \xi\left(\omega+\Delta_{j k}\right) e^{i \omega t}=\sqrt{S_{f}\left(\Delta_{j k}\right)} \xi_{\Delta_{j k}}(t)
$$

e aí o Hamiltoniano toma a forma

$$
H_{d e f}(t)=\sum_{j k}|j\rangle\langle k| \sigma_{z}^{j k} \sqrt{S_{f}\left(-\Delta_{j k}\right)} \xi_{-\Delta_{j k}}(t) .
$$

Esse Hamiltoniano permite escrever a equação de Born como

$$
\begin{aligned}
\frac{d \tilde{\rho}(t)}{d t}= & \int_{0}^{t} d \tau\left\{\sum_{j k j^{\prime} k^{\prime}} \sigma_{z}^{j k} \sigma_{z}^{j^{\prime} k^{\prime}}\left[\left|j^{\prime}\right\rangle\left\langle k^{\prime}|\tilde{\rho}(t-\tau)| j\right\rangle\langle k|-| j\rangle\left\langle k \mid j^{\prime}\right\rangle\left\langle k^{\prime}\right| \tilde{\rho}(t-\tau)\right] E\left[f(\omega) f\left(-\omega^{\prime}\right)\right]\right. \\
& \left.+\sum_{j k j^{\prime} k^{\prime}} \sigma_{z}^{j k} \sigma_{z}^{j^{\prime} k^{\prime}}\left[|j\rangle\left\langle k|\tilde{\rho}(t-\tau)| j^{\prime}\right\rangle\left\langle k^{\prime}|-\tilde{\rho}(t-\tau)| j^{\prime}\right\rangle\left\langle k^{\prime} \mid j\right\rangle\langle k|\right] E\left[f(\omega) f\left(-\omega^{\prime}\right)\right]\right\} .(3.65)
\end{aligned}
$$


Se for feita uma troca de índices $j$ por $k$ no primeiro somatório e $j$ ' por $k$ no segundo somatório, percebe-se que os únicos termos que não se anulam ocorrem quando $j^{\prime}=j$ e $k^{\prime}=k$.

$$
\begin{aligned}
\frac{d \tilde{\rho}(t)}{d t}= & \int_{0}^{t} d \tau\left\{\sum_{j k j^{\prime} k^{\prime}} \sigma_{z}^{j k} \sigma_{z}^{j^{\prime} k^{\prime}}\left[\left|j^{\prime}\right\rangle\left\langle k^{\prime}|\tilde{\rho}(t-\tau)| k\right\rangle\langle j|-| k\rangle\left\langle j \mid j^{\prime}\right\rangle\left\langle k^{\prime}\right| \tilde{\rho}(t-\tau)\right] E\left[f(\omega) f\left(-\omega^{\prime}\right)\right]\right. \\
& \left.+\sum_{j k j^{\prime} k^{\prime}} \sigma_{z}^{j k} \sigma_{z}^{j^{\prime} k^{\prime}}\left[|j\rangle\left\langle k|\tilde{\rho}(t-\tau)| k^{\prime}\right\rangle\left\langle j^{\prime}|-\tilde{\rho}(t-\tau)| k^{\prime}\right\rangle\left\langle j^{\prime} \mid j\right\rangle\langle k|\right] E\left[f(\omega) f\left(-\omega^{\prime}\right)\right]\right\} .(3.66)
\end{aligned}
$$

Diante disso, a contribuição para a equação mestra, ao se reorganizar os termos, será:

$$
\sum_{j, k \neq j}\left|\sigma_{z}^{j k}\right|^{2} \frac{\gamma_{\phi}\left(-\Delta_{j k}\right)}{2} \hat{\mathcal{D}}[|j\rangle\langle k|]+\frac{\gamma_{\phi}(0)}{2} \hat{\mathcal{D}}\left[\sum_{j} \sqrt{\frac{\gamma_{\phi}(0)}{2}} \sigma_{z}^{j j}|j\rangle\langle k|\right] .
$$

O termo $\gamma_{\phi}\left(-\Delta_{j k}\right)=2 S_{f}\left(-\Delta_{j k}\right)$ surgiu da correlação na equação (3.66).

\subsubsection{Modelo Quântico}

Nesta seção usaremos um modelo quântico para a descrição da defasagem, que leva a resultados similares aos da seção anterior. O procedimento será o mesmo adotado na seção sobre os banhos acoplados aos operadores $\sigma_{x}$ e $X$ : definir um Hamiltoniano de acoplamento, mudar para a representação de interação e, por fim, usar a equação (3.18).

O Hamiltoniano livre, com $b_{j}$ e $b_{j}^{\dagger}$ sendo os operadores escadas para o modo $j$ do banho tendo frequência $\nu_{j}$, é dado por:

$$
H_{B}=\sum_{j} \nu_{j} b_{j}^{\dagger} b_{j}
$$

O Hamiltoniano de interação entre o sistema e o banho é:

$$
H_{S B}=\sum_{j k} \alpha_{j k} b_{j}^{\dagger} b_{k} \sigma_{+} \sigma_{-}
$$

onde $\alpha_{j k}$ é a força de acoplamento. Como se sabe essa interação corresponde à transferência de um quantum de energia do modo do banho para outro através de uma excitação virtual do qubit. O passo seguinte é escrever o Hamiltoniano na representação de interação com $U=e^{-i\left(H_{S}+H_{B}\right) t}$ e inserir duas relações de fechamento

$$
\begin{aligned}
\tilde{H}(t) & =\sum_{j k} \sum_{m} \sum_{n} \alpha_{j k} b_{j}^{\dagger} b_{k} e^{i\left(\nu_{j}-\nu_{k}\right) t}|m\rangle\left\langle m\left|\sigma_{+} \sigma_{-}\right| n\right\rangle\langle n| e^{i \Delta_{m n} t} \\
& =\sum_{j k m n} \alpha_{j k} b_{j}^{\dagger} b_{k} e^{i\left(\nu_{j}-\nu_{k}\right) t} Z_{m n}|m\rangle\langle n| e^{i \Delta_{m n} t},
\end{aligned}
$$


com $Z_{m n}=\left\langle m\left|\sigma_{+} \sigma_{-}\right| n\right\rangle$. É possível observar que o valor médio térmico não será nulo quando os índices $j=k$, diferentemente do que ocorria nos banhos acoplados aos operadores $\sigma_{x}$ e $X$, onde a interação dos operadores do banho com o sistema tinha valor médio nulo no equilíbrio térmico (vide as equações (3.33)-(3.40)). A solução encontrada é inserir esses termos no Hamiltoniano total, chegando ao Hamiltoniano efetivo:

$$
H_{S}^{\prime}=H_{S}+\sum_{j m n} \alpha_{j j} b_{j}^{\dagger} b_{j} Z_{m n}|m\rangle\langle n| e^{i \Delta_{m n} t} .
$$

Como o banho está em equilíbrio térmico

$$
H_{S}^{\prime}=H_{S}+\sum_{j m n} \alpha_{j j} \bar{n}_{j}(T) Z_{m n}|m\rangle\langle n| e^{i \Delta_{m n} t} .
$$

A partir daqui, define-se o Hamiltoniano de interação de forma simplificada como:

$$
\tilde{H}(t)=B(t) s(t)
$$

com

$$
\begin{aligned}
s(t) & =\sum_{m n} Z_{m n}|m\rangle\langle n| e^{i \Delta_{m n} t}=\sum_{m n} Z_{m n}(t) \\
B(t) & =\sum_{j k} \alpha_{j k} b_{j}^{\dagger} b_{k} e^{i\left(\nu_{j}-\nu_{k}\right) t}
\end{aligned}
$$

e então reescreve-se a equação de Born usando os mesmos procedimentos das seções anteriores:

$$
\begin{aligned}
\frac{d \tilde{\rho}(t)}{d t}= & -\int_{0}^{t} d t^{\prime}\left\{\left[s(t) s\left(t^{\prime}\right) \tilde{\rho}\left(t^{\prime}\right)-s\left(t^{\prime}\right) \tilde{\rho}\left(t^{\prime}\right) s(t)\right]\left\langle B(t) B\left(t^{\prime}\right)\right\rangle_{R}\right. \\
& \left.+\left[\tilde{\rho}\left(t^{\prime}\right) s\left(t^{\prime}\right) s(t)-s(t) \tilde{\rho}\left(t^{\prime}\right) s\left(t^{\prime}\right)\right]\left\langle B\left(t^{\prime}\right) B(t)\right\rangle_{R}\right\} \\
= & -\int_{0}^{t} d t^{\prime} \sum_{m n m^{\prime} n^{\prime}}\left\{\left[Z_{m n}(t) Z_{m^{\prime} n^{\prime}}\left(t^{\prime}\right) \tilde{\rho}\left(t^{\prime}\right)-Z_{m^{\prime} n^{\prime}}\left(t^{\prime}\right) \tilde{\rho}\left(t^{\prime}\right) Z_{m n}(t)\right]\left\langle B(t) B\left(t^{\prime}\right)\right\rangle_{R}\right. \\
& \left.+\left[\tilde{\rho}\left(t^{\prime}\right) Z_{m^{\prime} n^{\prime}}\left(t^{\prime}\right) Z_{m n}(t)-Z_{m n}(t) \tilde{\rho}\left(t^{\prime}\right) Z_{m^{\prime} n^{\prime}}\left(t^{\prime}\right)\right]\left\langle B\left(t^{\prime}\right) B(t)\right\rangle_{R}\right\}
\end{aligned}
$$




$$
\begin{aligned}
& =-\int_{0}^{t} d \tau \sum_{m n m^{\prime} n^{\prime}}\left\{\left[Z_{m n}(t) Z_{m^{\prime} n^{\prime}}(t) e^{-i \Delta_{m^{\prime} n^{\prime}} \tau} \tilde{\rho}(t-\tau)-Z_{m^{\prime} n^{\prime}}(t) e^{-i \Delta_{m^{\prime} n^{\prime}} \tau} \tilde{\rho}(t-\tau) Z_{m n}(t)\right]\right. \\
& \times\langle B(t) B(t-\tau)\rangle_{R} \\
& \left.+\left[\tilde{\rho}(t-\tau) Z_{m^{\prime} n^{\prime}}(t) e^{-i \Delta_{m^{\prime} n^{\prime}} \tau} Z_{m n}(t)-Z_{m n}(t) \tilde{\rho}(t-\tau) Z_{m^{\prime} n^{\prime}}(t) e^{-i \Delta_{m^{\prime} n^{\prime}}}\right]\langle B(t-\tau) B(t)\rangle_{R}\right\} \\
& =-\sum_{m n m^{\prime} n^{\prime}}\left\{\left[Z_{m n}(t) Z_{m^{\prime} n^{\prime}}(t) \tilde{\rho}(t-\tau)-Z_{m^{\prime} n^{\prime}}(t) \tilde{\rho}(t-\tau) Z_{m n}(t)\right]\right. \\
& \times \int_{0}^{t} d \tau e^{-i \Delta_{m^{\prime} n^{\prime}} \tau}\langle B(t) B(t-\tau)\rangle_{R} \\
& +\left[\tilde{\rho}(t-\tau) Z_{m^{\prime} n^{\prime}}(t) Z_{m n}(t)-Z_{m n}(t) \rho_{I}(t-\tau) Z_{m^{\prime} n^{\prime}}(t)\right] \\
& \left.\times \int_{0}^{t} d \tau e^{-i \Delta_{m^{\prime} n^{\prime}} \tau}\langle B(t-\tau) B(t)\rangle_{R}\right\} \\
& =\left\{\sum _ { m n m ^ { \prime } n ^ { \prime } } \left[Z_{m^{\prime} n^{\prime}} Z_{m n}\left|m^{\prime}\right\rangle\left\langle n^{\prime}\left|e^{i \Delta_{m^{\prime} n^{\prime}} t} e^{i \Delta_{m n} t} \tilde{\rho}(t-\tau)\right| m\right\rangle\langle n|\right.\right. \\
& \left.-Z_{m n} Z_{m^{\prime} n^{\prime}} e^{i \Delta_{m n} t} e^{i \Delta_{m^{\prime} n^{\prime}} t}|m\rangle\left\langle n|| m^{\prime}\right\rangle\left\langle n^{\prime}\right| \tilde{\rho}(t-\tau)\right] \\
& \times \int_{0}^{t} d \tau e^{-i \Delta_{m^{\prime} n^{\prime}} \tau}\langle B(t) B(t-\tau)\rangle_{R} \\
& +\sum_{m n m^{\prime} n^{\prime}}\left[Z_{m n} Z_{m^{\prime} n^{\prime}} e^{i \Delta_{m n} t} e^{i \Delta_{m^{\prime} n^{\prime}} t}|m\rangle\left\langle n|\tilde{\rho}(t-\tau)| m^{\prime}\right\rangle\left\langle n^{\prime}\right|\right. \\
& \left.\left.-\tilde{\rho}(t-\tau)\left|m^{\prime}\right\rangle\left\langle n^{\prime}|| m\right\rangle\langle n| Z_{m^{\prime} n^{\prime}} Z_{m n} e^{i \Delta_{m^{\prime} n^{\prime}} t} e^{i \Delta_{m n} t}\right] \times \int_{0}^{t} d \tau e^{-i \Delta_{m^{\prime} n^{\prime}} \tau}\langle B(t-\tau) B(t)\rangle_{R}\right\} .
\end{aligned}
$$

Aqui será feita uma troca dos índices $m$ por $n$ no primeiro somatório e também de $m^{\prime}$ por $n^{\prime}$ no segundo somatório, obtendo

$$
\begin{aligned}
\frac{d \tilde{\rho}(t)}{d t}= & \left\{\sum _ { n m m ^ { \prime } n ^ { \prime } } \left[Z_{m^{\prime} n^{\prime}} Z_{n m}\left|m^{\prime}\right\rangle\left\langle n^{\prime}\left|e^{i \Delta_{m^{\prime} n^{\prime}} t} e^{i \Delta_{n m} t} \rho_{I}(t-\tau)\right| n\right\rangle\langle m|\right.\right. \\
& \left.-Z_{n m} Z_{m^{\prime} n^{\prime}} e^{i \Delta_{n m} t} e^{i \Delta_{m^{\prime} n^{\prime}} t}|n\rangle\left\langle m \mid m^{\prime}\right\rangle\left\langle n^{\prime}\right| \rho_{I}(t-\tau)\right] \times \int_{0}^{t} d \tau e^{-i \Delta_{m^{\prime} n^{\prime}} \tau}\langle B(t) B(t-\tau)\rangle \\
& +\sum_{m n n^{\prime} m^{\prime}}\left[Z_{m n} Z_{n^{\prime} m^{\prime}} e^{i \Delta_{m n} t} e^{i \Delta_{n^{\prime} m^{\prime}} t}|m\rangle\left\langle n\left|\rho_{I}(t-\tau)\right| n^{\prime}\right\rangle\left\langle m^{\prime}\right|\right. \\
& \left.\left.-\rho_{I}(t-\tau)\left|n^{\prime}\right\rangle\left\langle m^{\prime}|| m\right\rangle\langle n| Z_{n^{\prime} m^{\prime}} Z_{m n} e^{i \Delta_{n^{\prime} m^{\prime}} t} e^{i \Delta_{m n} t}\right] \times \int_{0}^{t} d \tau e^{-i \Delta_{n^{\prime} m^{\prime}} \tau}\langle B(t-\tau) B(t)\rangle\right\}
\end{aligned}
$$

Obtém-se, portanto, termos não nulos somente quando $m=m^{\prime}$ e $n=n^{\prime}$. Diante disso, usando 


$$
\begin{aligned}
\Delta_{m n}=- & \Delta_{n m}, \\
\frac{d \tilde{\rho}(t)}{d t}= & \left\{\sum_{m n}\left[Z_{m n} Z_{n m}|m\rangle\left\langle n\left|e^{i \Delta_{m n} t} e^{i \Delta_{n m} t} \tilde{\rho}(t-\tau)\right| n\right\rangle\left\langle m\left|-Z_{n m} Z_{m n} e^{i \Delta_{n m} t} e^{i \Delta_{m n} t}\right| n\right\rangle\langle n| \tilde{\rho}(t-\tau)\right]\right. \\
& \times \int_{0}^{t} d \tau e^{-i \Delta_{m n} \tau}\langle B(t) B(t-\tau)\rangle_{R} \\
& +\sum_{m n}\left[Z_{m n} Z_{n m} e^{i \Delta_{m n} t} e^{i \Delta_{n m} t}|m\rangle\langle n|\tilde{\rho}(t-\tau)| n\rangle\langle m|-\tilde{\rho}(t-\tau)| n\rangle\langle n| Z_{n m} Z_{m n} e^{i \Delta_{n m} t} e^{i \Delta_{m n} t}\right] \\
& \left.\times \int_{0}^{t} d \tau e^{-i \Delta_{n m} \tau}\langle B(t-\tau) B(t)\rangle_{R}\right\} \\
= & \left\{\sum_{m n}\left[\left|Z_{m n}\right|^{2}|m\rangle\langle n|\tilde{\rho}(t-\tau)| n\rangle\left\langle\left. m|-| Z_{m n}\right|^{2} \mid n\right\rangle\langle n| \tilde{\rho}(t-\tau)\right]\right. \\
& \times \int_{0}^{t} d \tau e^{-i \Delta_{m n} \tau}\langle B(t) B(t-\tau)\rangle_{R} \\
& +\sum_{m n}\left[\left|Z_{m n}\right|^{2}|m\rangle\langle n|\tilde{\rho}(t-\tau)| n\rangle\left\langle\left. m|-| Z_{m n}\right|^{2} \tilde{\rho}(t-\tau) \mid n\right\rangle\langle n|\right] \\
& \left.\times \int_{0}^{t} d \tau e^{-i \Delta_{n m} \tau}\langle B(t-\tau) B(t)\rangle_{R}\right\}
\end{aligned}
$$

As funções de correlação têm como resultado

$$
\begin{aligned}
\langle B(t) B(t-\tau)\rangle_{R} & =\sum_{j \neq k} \sum_{j^{\prime} \neq k^{\prime}} \alpha_{j k} \alpha_{j^{\prime} k^{\prime}}\left\langle b_{j}^{\dagger} b_{k} b_{j^{\prime}}^{\dagger} b_{k^{\prime}}\right\rangle_{R} e^{i\left(\nu_{j}-\nu_{k}\right) t} e^{i\left(\nu_{j^{\prime}}-\nu_{k^{\prime}}\right) t^{\prime}}, \\
\langle B(t-\tau) B(t)\rangle_{R} & =\sum_{j \neq k} \sum_{j^{\prime} \neq k^{\prime}} \alpha_{j k} \alpha_{j^{\prime} k^{\prime}}\left\langle b_{j^{\prime}}^{\dagger} b_{k^{\prime}} b_{j}^{\dagger} b_{k}\right\rangle_{R} e^{i\left(\nu_{j^{\prime}}-\nu_{k^{\prime}}\right) t^{\prime}} e^{i\left(\nu_{j}-\nu_{k}\right) t} .
\end{aligned}
$$

Em ambos os casos, o valor médio só não anula quando $j=k^{\prime}$ e $j^{\prime}=k$, pois para o estado térmico $\left\langle b_{j}^{\dagger} b_{k \neq j}\right\rangle=0$. Portanto, sendo $\alpha_{k j}=\alpha_{j k}$

$$
\begin{aligned}
\langle B(t) B(t-\tau)\rangle_{R} & =\sum_{j \neq k} \alpha_{j k} \alpha_{k j}\left\langle b_{j}^{\dagger} b_{k} b_{k}^{\dagger} b_{j}\right\rangle_{R} e^{i\left(v_{j}-v_{k}\right) t} e^{i\left(v_{k}-v_{j}\right)(t-\tau)} \\
& =\sum_{j \neq k} \alpha_{j k}^{2} \bar{n}_{j}(T)\left[\bar{n}_{k}(T)+1\right] e^{i\left(v_{j}-v_{k}\right) \tau}, \\
\langle B(t-\tau) B(t)\rangle_{R} & =\sum_{j \neq k} \alpha_{j k} \alpha_{k j}\left\langle b_{k}^{\dagger} b_{j} b_{j}^{\dagger} b_{k}\right\rangle_{R} e^{i\left(v_{k}-v_{j}\right)(t-\tau)} e^{i\left(v_{j}-v_{k}\right) t} \\
& =\sum_{j \neq k} \alpha_{j k}^{2} \bar{n}_{j}(T)\left[\bar{n}_{k}(T)+1\right] e^{-i\left(v_{j}-v_{k}\right) \tau} .
\end{aligned}
$$


Esse resultado nos permite resolver as integrais na equação (3.76) da mesma forma que na seção 3.2

$$
\begin{aligned}
\lim _{t \rightarrow \infty} \int_{0}^{t} d \tau e^{i\left(v^{\prime}-v-\Delta_{m n}\right) \tau} & =\lim _{t \rightarrow \infty} \int_{0}^{t} d \tau e^{-i\left(-v^{\prime}+v-\Delta_{n m}\right) \tau}=\pi \delta\left(-v^{\prime}+v-\Delta_{n m}\right) \\
-i \frac{\mathcal{P}}{-v^{\prime}+v-\Delta_{n m}} & =\pi \delta\left(v^{\prime}-v+\Delta_{n m}\right)+i \frac{\mathcal{P}}{v^{\prime}-v+\Delta_{n m}}, \\
\lim _{t \rightarrow \infty} \int_{0}^{t} d \tau e^{-i\left(v^{\prime}-v-\Delta_{m n}\right) \tau} & =\pi \delta\left(v^{\prime}-v+\Delta_{n m}\right)-i \frac{\mathcal{P}}{v^{\prime}-v+\Delta_{n m}} .
\end{aligned}
$$

Da mesma forma que na seção 3.2, estendendo o somatório à uma integral introduzindo a densidade de estados $D(v)$, obtém-se

$$
\begin{aligned}
\int_{0}^{t} d \tau e^{-i \Delta_{m n} \tau}\langle B(t) B(t-\tau)\rangle_{R}= & {\left[\pi \delta\left(v^{\prime}-v+\Delta_{n m}\right)+i \frac{\mathcal{P}}{v^{\prime}-v+\Delta_{n m}}\right] } \\
& \times \int_{0}^{\infty} d v d v^{\prime} \alpha^{2}\left(v, v^{\prime}\right) D(v) D\left(v^{\prime}\right) \bar{n}(v, T)\left[\bar{n}\left(v^{\prime}, T\right)+1\right] \\
= & \pi \int_{0}^{\infty} d v \alpha^{2}\left(v, v-\Delta_{n m}\right) D(v) D\left(v-\Delta_{n m}\right) \bar{n}(v, T) \\
& \times\left[\bar{n}\left(v-\Delta_{n m}, T\right)+1\right] \\
& +i \mathcal{P} \int_{0}^{\infty} \frac{d v d v^{\prime} \alpha^{2}\left(v, v^{\prime}\right) D(v) D\left(v^{\prime}\right) \bar{n}(v, T)\left[\bar{n}\left(v^{\prime}, T\right)+1\right]}{v^{\prime}-v+\Delta_{n m}}, \\
\int_{0}^{t} d \tau e^{-i \Delta_{n m} \tau}\langle B(t-\tau) B(t)\rangle_{R}= & {\left[\pi \delta\left(v^{\prime}-v+\Delta_{n m}\right)-i \frac{\mathcal{P}}{v^{\prime}-v+\Delta_{n m}}\right] } \\
& \times \int_{0}^{\infty} d v d v^{\prime} \alpha^{2}\left(v, v^{\prime}\right) D(v) D\left(v^{\prime}\right) \bar{n}(v, T)\left[\bar{n}\left(v^{\prime}, T\right)+1\right] \\
= & \pi \int_{0}^{\infty} d v \alpha^{2}\left(v, v-\Delta_{n m}\right) D(v) D\left(v-\Delta_{n m}\right) \bar{n}(v, T) \\
& \times\left[\bar{n}\left(v-\Delta_{n m}, T\right)+1\right] \\
& -i \mathcal{P} \int_{0}^{\infty} \frac{d v d v^{\prime} \alpha^{2}\left(v, v^{\prime}\right) D(v) D\left(v^{\prime}\right) \bar{n}(v, T)\left[\bar{n}\left(v^{\prime}, T\right)+1\right]}{v^{\prime}-v+\Delta_{n m}} .
\end{aligned}
$$

Definindo

$$
\begin{aligned}
\gamma_{n m} & =2 \pi \int_{0}^{\infty} d v \alpha^{2}\left(v, v-\Delta_{n m}\right) D(v) D\left(v-\Delta_{n m}\right) \bar{n}(v, T)\left[\bar{n}\left(v-\Delta_{n m}, T\right)+1\right], \\
L_{m n} & =\mathcal{P} \int_{0}^{\infty} \frac{d v d v^{\prime} \alpha^{2}\left(v, v^{\prime}\right) D(v) D\left(v^{\prime}\right) \bar{n}(v, T)\left[\bar{n}\left(v^{\prime}, T\right)+1\right]}{v^{\prime}-v+\Delta_{n m}}
\end{aligned}
$$


então, o resultado será:

$$
\begin{aligned}
\frac{d \tilde{\rho}(t)}{d t}= & \sum_{m n} \frac{\gamma_{n m}}{2}\left|Z_{m n}\right|^{2}\left[|m\rangle\langle n|\tilde{\rho}(t)| n\rangle\langle m|+| m\rangle\left\langle n\left|\rho_{I}(t)\right| n\right\rangle\langle m|-| n\rangle\langle n|\tilde{\rho}(t)-\tilde{\rho}(t)| n\rangle\langle n|\right] \\
& +\sum_{m n} i L_{m n}\left|Z_{m n}\right|^{2}[|m\rangle\langle n|\tilde{\rho}(t)| n\rangle\langle m|-| m\rangle\langle n|\tilde{\rho}(t)| n\rangle\langle m|+\tilde{\rho}(t)| n\rangle\langle n|-| n\rangle\langle n| \tilde{\rho}(t)] .
\end{aligned}
$$

Assim, voltando ao formalismo de Schrödinger, definindo $\gamma_{\phi}\left(\Delta_{m n}\right)=\gamma_{m n} / 2$ e usando a relação $Z_{m n}=\left(\delta_{m n}+\sigma_{z}^{m n}\right) / 2$, o resultado será

$$
\begin{aligned}
\dot{\rho}(t)= & -i\left[H_{S}^{\prime}, \rho(t)\right]+\sum_{m n} i L_{m n}\left|Z_{m n}\right|^{2}[\rho(t)|n\rangle\langle n|-| n\rangle\langle n| \rho(t)] \\
& +\frac{\gamma_{\phi}(0)}{2} \sum_{m}\left|\sigma_{z}^{m m}\right|^{2}\left[|m\rangle\langle m|\rho(t)| m\rangle\left\langle m\left|-\frac{1}{2}\right| m\right\rangle\left\langle m\left|\rho(t)-\frac{1}{2} \rho(t)\right| m\right\rangle\langle m|\right] \\
& +\frac{\gamma_{\phi}\left(\Delta_{n m}\right)}{2} \sum_{m m \neq n}\left|\sigma_{z}^{m n}\right|^{2}\left[|m\rangle\langle n|\rho(t)| n\rangle\left\langle m\left|-\frac{1}{2}\right| n\right\rangle\left\langle n\left|\rho(t)-\frac{1}{2} \rho(t)\right| n\right\rangle\langle n|\right] \\
= & -i\left[H_{S}^{\prime \prime}, \rho(t)\right]+\frac{\gamma_{\phi}(0)}{2} \hat{\mathcal{D}}\left[\sum_{m} \sigma_{z}^{m m}|m\rangle\langle m|\right] \rho(t)+\frac{\gamma_{\phi}\left(\Delta_{n m}\right)}{2} \sum_{m n}\left|\sigma_{z}^{m n}\right|^{2} \hat{\mathcal{D}}[|m\rangle\langle n|] \rho(t)
\end{aligned}
$$

onde $H_{S}^{\prime \prime}=H_{S}^{\prime}+\sum_{m n} i\left|Z_{m n}\right|^{2} L_{m n}|n\rangle\langle n|$ é o Hamiltoniano Lamb-shifted. Repare que se forem desprezados os Lamb shifts, será obtida a mesma equação mestra que no caso clássico. Como o reservatório foi tomado desde o príncipio em equilíbrio térmico, o estado de Gibbs $\rho_{R}=\frac{e^{-\beta H_{B}}}{\operatorname{Tr}_{s}\left(e^{\left.-\beta H_{B}\right)}\right.}, \operatorname{com} \beta=\frac{1}{k_{B} T}$, é um estado estacionário. O estado estacionário tem como propriedade

$$
\sum_{n^{\prime}} W_{n n^{\prime}} P_{n^{\prime}}=\sum_{n^{\prime}} W_{n^{\prime} n} P_{n}
$$

onde $P_{n}=\left\langle n\left|\rho_{R}\right| n\right\rangle$ são as populações e $W_{n n^{\prime}}$ são taxas de emissão ou absorção entre os estados. Essa relação expressa o fato óbvio de que, em um estado estacionário, a soma de todas as transições por unidade de tempo para um estado $n$ deve ser balanceada pela soma de todas as transições de $n$ para outros estados n'. A relação conhecida como relação de Kubo-Martin-Schwinger (KMS) [29], fornece uma importante relação $[23,24]$

$$
\frac{\gamma_{\phi}(\omega)}{\gamma_{\phi}(-\omega)}=e^{\omega / k_{B} T} .
$$

A relação anterior é a importante afirmação de que para cada par de estados a transição deve ser balanceada e a razão entre as taxas de transição é proporcional a diferença de energia entre os estados. Tal relação é conhecida como balanço detalhado. O tratamento quântico feito nesta seção engloba 
a temperatura respeitando o balanço detalhado [16], isso implica que para temperatura $T=0$, $\gamma_{\phi}(\omega)=0$ para $\omega<0$. Entretanto, essa condição não requer conhecimento detalhado do banho, mas somente propriedades termodinâmicas. Em particular, não é necessário invocar a Mecânica Quântica (ver [28]). Essa relação de balanço detalhado pode ser demonstrada em Física Clássica para um sistema em contato com um banho térmico à temperatura $T$ através da relação

$$
W_{n n^{\prime}} e^{-\varepsilon_{n^{\prime}} / k_{B} T}=W_{n^{\prime} n} e^{-\varepsilon_{n} / k_{B} T},
$$

onde $\varepsilon_{n}$ é a energia do estado $n$. A quebra do balanço detalhado implica em aumento da entropia termodinâmica (irreversibilidade). 


\section{Capítulo 4}

\section{Considerações sobre a Equação Mestra Padrão}

Até o momento, foi deduzida uma descrição analítica (sob uma série de aproximações) de como a modulação externa com frequência $\eta^{(j)}$, de acordo com a frequência ressonante do sistema átomo-campo, permite obter efeitos de criação e aniquilação de fótons. Além disso, uma formulação mais completa para a equação mestra foi obtida considerando o acoplamento qubit-cavidade na base dos EV. Entretanto, quando os subsistemas são tratados separadamente, a formulação é feita em torno de Estados Despidos, que se revestem ou de átomo ou de campo. Devido a isso, operá-la sobre o operador densidade total é, em princípio, menos trabalhoso, bastando apenas que façamos certas aproximações justificadas.

O interesse agora é entender como a dissipação afeta a evolução temporal de tais fenômenos não-estacionários. Para tanto, será considerado um único qubit interagindo com o campo eletromagnético, de modo que os estados vestidos do sistema corresponderão aos autoestados do Hamiltoniano de Jaynes-Cummings [8, 19] (Apêndice D)

$$
H_{J C}=\omega_{0} n+\Omega_{0}|e\rangle\langle e|+g_{0}\left(a \sigma_{+}+a^{\dagger} \sigma_{-}\right),
$$

com $\omega_{0}$ sendo a frequência do campo, $\Omega_{0}$ a frequência de transição atômica e $g_{0}$ o termo de acoplamento átomo-campo. Os seus autovalores são dados por

$$
\lambda_{0}=0, \lambda_{n, \mathcal{S}}=\omega_{0} n-\frac{\Delta_{-}}{2}+\mathcal{S} \frac{1}{2} \beta_{n},
$$


com

$$
\Delta_{-}=\omega_{0}-\Omega_{0}
$$

sendo o termo de dissintonia entre átomo e campo, $\beta_{n}=\sqrt{\Delta_{-}^{2}+4 g_{0}^{2} n}=\lambda_{n,+}-\lambda_{n,-}$. O índice $\mathcal{S}= \pm$ é definido para diferenciar os autovalores com mesmo $n$, com $n$ sendo o número total de excitações do sistema. Com relação aos autoestados, tem-se

$$
\begin{gathered}
\left|\varphi_{0}\right\rangle=|g, 0\rangle \\
\left|\varphi_{n, \mathcal{S}}\right\rangle=\left(\mathrm{s}_{n, \mathcal{S}}|g, n\rangle+\mathrm{c}_{n, \mathcal{S}}|e, n-1\rangle\right),
\end{gathered}
$$

obtidos no apêndice $\mathrm{D}$, sendo

$$
\begin{aligned}
& \mathrm{s}_{m,+}=\sin \theta_{m}, \\
& \mathrm{~s}_{m,-}=\cos \theta_{m}, \\
& \mathrm{c}_{m,+}=\cos \theta_{m}, \\
& \mathrm{c}_{m,-}=-\sin \theta_{m} .
\end{aligned}
$$

Ainda, o termo $\theta_{n>0}=\arctan \frac{\Delta_{-}+\beta_{n}}{2 g_{0} \sqrt{n}}$ tem importância devido à sua dependência com o termo de dissintonia, que define tanto o Regime Ressonante (RR) quanto o Regime Dispersivo (RD). O RR é obtido quando a comparação entre a frequência de acoplamento $g_{0}$ para o sistema átomo-campo e o termo de dissintonia $\Delta_{-}$fornece $\left|\Delta_{-}\right| \ll g_{0}$. Por outro lado, o RD será obtido quando a mesma comparação fornece $g_{0} \sqrt{n} /\left|\Delta_{-}\right| \ll 1$ para todos os valores relevantes de $n$.

Por formalidade, é definido que $\left|\varphi_{0}\right\rangle=\left|\varphi_{0,-}\right\rangle \operatorname{com} \theta_{0}=0$, mas podemos ver que $\left|\varphi_{0,+}\right\rangle=$ $|e,-1\rangle$, que obviamente é não-físico, então, definimos que $\left|\varphi_{0,+}\right\rangle \equiv 0$. Além disso, para o RR $\theta_{n>0}=\pi / 4$, enquanto no RD duas situações são possíveis: $\Delta_{-}>0$ e $n>0$ ou $\Delta_{-}<0$ e $n>0$. Para $\Delta_{-}>0$ os estados vestidos até primeira ordem em $\frac{g_{0}}{\Delta_{-}} \sqrt{n}$ são (obtidos através da expansão em série dos resultados do apêndice D)

$$
\begin{aligned}
\left|\varphi_{n,+}\right\rangle & \approx|\mathbf{g}, n\rangle+\frac{g_{0}}{\Delta_{-}} \sqrt{n}|\mathbf{e}, n-1\rangle, \\
\left|\varphi_{n,-}\right\rangle & \approx \frac{g_{0}}{\Delta_{-}} \sqrt{n}|\mathbf{g}, n\rangle-|\mathbf{e}, n-1\rangle,
\end{aligned}
$$


com os coeficientes expandidos até segunda ordem

$$
\begin{aligned}
\sin ^{2} \theta_{n} & \approx 1-\frac{g_{0}^{2}}{\Delta_{-}^{2}} n+\frac{g_{0}^{4}}{\Delta_{-}^{4}} n^{2}, \sin \theta_{n} \approx 1-\frac{1}{2} \frac{g_{0}^{2}}{\Delta_{-}^{2}} n+\frac{1}{2} \frac{g_{0}^{4}}{\Delta_{-}^{4}} n^{2} \\
\cos ^{2} \theta_{n} & =\frac{g_{0}^{2}}{\Delta_{-}^{2}} n-\frac{g_{0}^{4}}{\Delta_{-}^{4}} n^{2}, \cos \theta_{n} \approx \frac{g_{0}}{\Delta_{-}} \sqrt{n}\left(1-\frac{1}{2} \frac{g_{0}^{2}}{\Delta_{-}^{2}} n\right) \\
\sin 2 \theta_{n} & \approx 2 \frac{g_{0}}{\Delta_{-}} \sqrt{n}, \sin ^{2} 2 \theta_{n} \approx 4 \frac{g_{0}^{2}}{\Delta_{-}^{2}} n \\
\cos 2 \theta_{n} & \approx-1+2 \frac{g_{0}^{2}}{\Delta_{-}^{2}} n, \cos ^{2} 2 \theta_{n} \approx 1-4 \frac{g_{0}^{2}}{\Delta_{-}^{2}} n .
\end{aligned}
$$

Já para $\Delta_{-}<0$, os estados vestidos até primeira ordem em $\frac{g_{0}}{\Delta_{-}} \sqrt{n}$ são

$$
\begin{aligned}
\left|\varphi_{n,+}\right\rangle & \approx-\frac{g_{0}}{\Delta_{-}} \sqrt{n}|\mathbf{g}, n\rangle+|\mathbf{e}, n-1\rangle, \\
\left|\varphi_{n,-}\right\rangle & \approx|\mathbf{g}, n\rangle+\frac{g_{0}}{\Delta_{-}} \sqrt{n}|\mathbf{e}, n-1\rangle,
\end{aligned}
$$

com os coeficientes obtidos até segunda ordem

$$
\begin{aligned}
\sin ^{2} \theta_{n} & \approx \frac{g_{0}^{2}}{\Delta_{-}^{2}} n-\frac{g_{0}^{4}}{\Delta_{-}^{4}} n^{2}, \sin \theta_{n} \approx-\frac{g_{0}}{\Delta_{-}} \sqrt{n}\left(1-\frac{1}{2} \frac{g_{0}^{2}}{\Delta_{-}^{2}} n\right): \\
\cos ^{2} \theta_{n} & \approx 1-\frac{g_{0}^{2}}{\Delta_{-}^{2}} n+\frac{g_{0}^{4}}{\Delta_{-}^{4}} n^{2}, \cos \theta_{n} \approx 1-\frac{1}{2} \frac{g_{0}^{2}}{\Delta_{-}^{2}} n+\frac{1}{2} \frac{g_{0}^{4}}{\Delta_{-}^{4}} n^{2} ; \\
\sin 2 \theta_{n} & \approx-\frac{2 g_{0}}{\Delta_{-}} \sqrt{n}, \cos 2 \theta_{n}=1-2 \frac{g_{0}^{2}}{\Delta_{-}^{2}} n \\
\sin ^{2} 2 \theta_{n} & \approx \frac{4 g_{0}^{2}}{\Delta_{-}^{2}} n, \cos ^{2} 2 \theta_{n}=1-4 \frac{g_{0}^{2}}{\Delta_{-}^{2}} n .
\end{aligned}
$$

No RD, os autoestados expandindos até primeira ordem em $\frac{g_{0}}{\Delta_{-}} \sqrt{n}$ podem ser generalizados de acordo com o símbolo de dissintonia $\mathcal{D}=\Delta_{-} /\left|\Delta_{-}\right|$, que facilita a notação, sendo dados por

$$
\begin{aligned}
\left|\varphi_{n, \mathcal{D}}\right\rangle & \approx|\mathbf{g}, n\rangle+\frac{g_{0}}{\Delta_{-}} \sqrt{n}|\mathbf{e}, n-1\rangle, \\
\left|\varphi_{n,-\mathcal{D}}\right\rangle & \approx-\mathcal{D}\left[|\mathbf{e}, n-1\rangle-\frac{g_{0}}{\Delta_{-}} \sqrt{n}|\mathbf{g}, n\rangle\right] .
\end{aligned}
$$

Os EV tomados em ordem zero no RD são dados aproximadamente por

$$
\left|\varphi_{n, \mathcal{D}}\right\rangle \approx|\mathbf{g}, n\rangle ;\left|\varphi_{n,-\mathcal{D}}\right\rangle \approx|\mathbf{e}, n-1\rangle .
$$

Esse formalismo será importante no estudo da Equação Mestra Padrão (EMP), pois serão abordados efeitos tanto no RR quanto no RD. Entretanto, no RD serão consideradas no máximo expansões em segunda ordem. 


\subsection{Equação Mestra Padrão}

Na presença de dissipação, a dinâmica do sistema é descrita pela equação mestra

$$
\frac{d \hat{\rho}}{d t}=-i[\hat{H}, \hat{\rho}]+\hat{\mathcal{L}} \hat{\rho}
$$

sendo $\hat{\mathcal{L}}$ o superoperador Liouvilliano. Muito conhecida na literatura e já apresentada, a equação mestra padrão à temperatura zero é definida por (1.2)

$$
\hat{\mathcal{L}}_{E M P} \bullet=\kappa \hat{\mathcal{D}}[\hat{a}] \bullet+\gamma \hat{\mathcal{D}}\left[\hat{\sigma}_{-}\right] \bullet+\frac{\gamma_{\phi}}{2} \hat{\mathcal{D}}\left[\hat{\sigma}_{z}\right] \bullet
$$

$\operatorname{com} \mathcal{D}[\hat{O}] \hat{\rho}=\frac{1}{2}\left(\hat{O} \hat{\rho} \hat{O}^{\dagger}-\hat{O}^{\dagger} \hat{O} \hat{\rho}-\hat{\rho} \hat{O}^{\dagger} \hat{O}\right)$ sendo o superoperador Lindbladiano e $\hat{\sigma}_{z}=|e\rangle\langle e|-| g\rangle\langle g|$. Os parâmetros $\kappa, \gamma$ e $\gamma_{\phi}$ são, respectivamente, as taxas de relaxação da cavidade, relaxamento do qubit e defasagem pura do qubit.

$\mathrm{Na}$ representação de interação os operadores serão reescritos através de $U=e^{-i t H_{J C}}$, sendo obtido o operador densidade $\rho=U \tilde{\rho} U^{\dagger}$ e qualquer operador como $\tilde{O}=e^{i t H_{J C}} O e^{-i t H_{J C}}$ [47]. Logo, a EMP

$$
\begin{aligned}
\dot{\rho}= & -i[H, \rho]+\frac{\kappa}{2}\left(2 a \rho a^{\dagger}-a^{\dagger} a \rho-\rho a^{\dagger} a\right)+\frac{\gamma}{2}\left(2 \sigma_{-} \rho \sigma_{+}-\sigma_{+} \sigma_{-} \rho-\rho \sigma_{+} \sigma_{-}\right) \\
& +\frac{\gamma_{\phi}}{2}\left(\sigma_{z} \rho \sigma_{z}-\rho\right)
\end{aligned}
$$

na representação de interação se torna

$$
\begin{aligned}
\frac{d \tilde{\rho}}{d t}= & -i[\tilde{H}, \tilde{\rho}]+\frac{\kappa}{2}\left(2 \tilde{a} \tilde{\rho} \tilde{a}^{\dagger}-\tilde{a}^{\dagger} \tilde{a} \tilde{\rho}-\tilde{\rho} \tilde{a}^{\dagger} \tilde{a}\right)+\frac{\gamma}{2}\left(2 \tilde{\sigma}_{-} \tilde{\rho} \tilde{\sigma}_{+}-\tilde{\sigma}_{+} \tilde{\sigma}_{-} \tilde{\rho}-\tilde{\rho} \tilde{\sigma}_{+} \tilde{\sigma}_{-}\right) \\
& +\frac{\gamma_{\phi}}{2}\left(\tilde{\sigma}_{z} \tilde{\rho} \tilde{\sigma}_{z}-\tilde{\rho}\right) .
\end{aligned}
$$

Além disso, o operador densidade será expadido na base dos estados vestidos

$$
\begin{aligned}
\tilde{\rho} & =\sum_{\mathcal{S}, \mathcal{T}= \pm} \sum_{n, m=0}^{\infty} \tilde{\rho}_{n, m}^{\mathcal{S}, \mathcal{T}}(t)\left|\varphi_{n, \mathcal{S}}\right\rangle\left\langle\varphi_{m, \mathcal{T}}\right| \\
& =\sum_{n, m=0}^{\infty}\left\{\tilde{\rho}_{n, m}^{+,+}\left|\varphi_{n,+}\right\rangle\left\langle\varphi_{m,+}\left|+\tilde{\rho}_{n, m}^{-,-}\right| \varphi_{n,-}\right\rangle\left\langle\varphi_{m,-}\left|+\tilde{\rho}_{n, m}^{+,-}\right| \varphi_{n,+}\right\rangle\left\langle\varphi_{m,-}\left|+\left(\tilde{\rho}_{m, n}^{+,-}\right)^{*}\right| \varphi_{n,-}\right\rangle\left\langle\varphi_{m,+}\right|\right\},
\end{aligned}
$$

observado-se que $\tilde{\rho}_{n, m}^{+,+}=\left(\tilde{\rho}_{m, n}^{+,+}\right)^{*}, \tilde{\rho}_{n, m}^{-,-}=\left(\tilde{\rho}_{m, n}^{-,-}\right)^{*}, \tilde{\rho}_{n, m}^{-,+}=\left(\tilde{\rho}_{m, n}^{+,-}\right)^{*}$ e $\mathcal{S}, \mathcal{T}= \pm$ (índices mudos). 


\subsection{Simplificações para EMP}

De posse das definições à respeito da EMP na representação de interação (a mesma usada no capítulo 2) e do operador densidade expandido em termos dos EV, pode-se obter as equações diferenciais para $\tilde{\rho}_{n, m}^{\mathcal{S}, \mathcal{T}}(t)$. É interessante escrever "individualmente" os três termos de (4.12), de modo que se possa analisar como cada efeito no RR e no RD será afetado pela relaxação atômica pura, defasagem atômica pura e relaxação pura da cavidade. Ainda, algumas simplificações serão fundamentais para a análise do comportamento assintótico do sistema sob a dissipação. Assim, para a relaxação atômica pura

$$
\begin{aligned}
& \frac{d}{d t} \sum_{N, M=0}^{\infty}\left\{\tilde{\rho}_{N M}^{+,+}\left|\varphi_{N,+}\right\rangle\left\langle\varphi_{M,+}\left|+\rho_{N M}^{-,-}\right| \varphi_{N,-}\right\rangle\left\langle\varphi_{M,-}\left|+\tilde{\rho}_{N M}^{+,-}\right| \varphi_{N,+}\right\rangle\left\langle\varphi_{M,-}\left|+\left(\tilde{\rho}_{M N}^{+,-}\right)^{*}\right| \varphi_{N,-}\right\rangle\left\langle\varphi_{M,+}\right|\right\} \\
= & \frac{\gamma}{2}\left(2 \tilde{\sigma}_{-} \tilde{\rho} \tilde{\sigma}_{+}-\tilde{\sigma}_{+} \tilde{\sigma}_{-} \tilde{\rho}-\tilde{\rho} \tilde{\sigma}_{+} \tilde{\sigma}_{-}\right),
\end{aligned}
$$

bastando projetar do lado esquerdo o bra $\left\langle\varphi_{N,+}\right|$ e do lado direito o ket $\left|\varphi_{M,+}\right\rangle$ para ter a equação diferencial para $\tilde{\rho}_{N M}^{+,+}$

$$
\frac{d}{d t} \tilde{\rho}_{N M}^{+,+}=\frac{\gamma}{2}\left\langle\varphi_{N,+}\left|\left(2 \tilde{\sigma}_{-} \tilde{\rho} \tilde{\sigma}_{+}-\tilde{\sigma}_{+} \tilde{\sigma}_{-} \tilde{\rho}-\tilde{\rho} \tilde{\sigma}_{+} \tilde{\sigma}_{-}\right)\right| \varphi_{M,+}\right\rangle ;
$$

de forma semelhante, apenas atuando do lado esquerdo $\left\langle\varphi_{N,-}\right|$ e do direito $\left|\varphi_{M,-}\right\rangle$

$$
\frac{d}{d t} \tilde{\rho}_{N M}^{-,-}=\frac{\gamma}{2}\left\langle\varphi_{N,-}\left|\left(2 \tilde{\sigma}_{-} \tilde{\rho} \tilde{\sigma}_{+}-\tilde{\sigma}_{+} \tilde{\sigma}_{-} \tilde{\rho}-\tilde{\rho} \tilde{\sigma}_{+} \tilde{\sigma}_{-}\right)\right| \varphi_{M,-}\right\rangle ;
$$

enquanto $\tilde{\rho}_{n, m}^{-,+}=\left(\tilde{\rho}_{m, n}^{+,-}\right)^{*}$, basta escrever a equação diferencial para $\tilde{\rho}_{N M}^{+,-}$

$$
\frac{d}{d t} \tilde{\rho}_{N M}^{+,-}=\frac{\gamma}{2}\left\langle\varphi_{N,+}\left|\left(2 \tilde{\sigma}_{-} \tilde{\rho} \tilde{\sigma}_{+}-\tilde{\sigma}_{+} \tilde{\sigma}_{-} \tilde{\rho}-\tilde{\rho} \tilde{\sigma}_{+} \tilde{\sigma}_{-}\right)\right| \varphi_{M,-}\right\rangle .
$$

Através da aplicação direta dos operadores no operador densidade, as equações diferenciais para a relaxação atômica pura serão

$$
\begin{aligned}
\frac{d}{d t} \tilde{\rho}_{N, M}^{+,+}= & \frac{\gamma}{2}\left\{2 \operatorname { s i n } \theta _ { N } \operatorname { s i n } \theta _ { M } e ^ { i t ( \lambda _ { N , + } - \lambda _ { M , + } ) } \left[\cos \theta_{N+1} \cos \theta_{M+1} e^{-i t\left(\lambda_{N+1,+}-\lambda_{M+1,+}\right)} \tilde{\rho}_{N+1, M+1}^{+,+}(4,20)\right.\right. \\
& -\sin \theta_{M+1} \cos \theta_{N+1} e^{-i t\left(\lambda_{N+1,+}-\lambda_{M+1,-}\right)} \tilde{\rho}_{N+1, M+1}^{+,-}-\sin \theta_{N+1} \cos \theta_{M+1} \\
& \left.\times e^{i t\left(\lambda_{M+1,+}-\lambda_{N+1,-}\right)}\left(\tilde{\rho}_{M+1, N+1}^{+,-}\right)^{*}+\sin \theta_{N+1} \sin \theta_{M+1} e^{-i t\left(\lambda_{N+1,-}-\lambda_{M+1,-}\right)} \tilde{\rho}_{N+1, M+1}^{-,-}\right] \\
& -\left(\cos ^{2} \theta_{N}+\cos ^{2} \theta_{M}\right) \tilde{\rho}_{N M}^{+,+} \\
& \left.\left.+\frac{1}{2}\left(\sin 2 \theta_{M} e^{-i t\left(\lambda_{M,+}-\lambda_{M,-}\right)} \tilde{\rho}_{N M}^{+,-}+\sin 2 \theta_{N} e^{i t\left(\lambda_{N,+}-\lambda_{N,-}\right.}\right)\left(\tilde{\rho}_{M, N}^{+,-}\right)^{*}\right)\right\}
\end{aligned}
$$




$$
\begin{aligned}
& \frac{d}{d t} \tilde{\rho}_{N M}^{-,-}= \frac{\gamma}{2}\left\{2 \operatorname { c o s } \theta _ { N } \operatorname { c o s } \theta _ { M } e ^ { i t ( \lambda _ { N , - } - \lambda _ { M , - } ) } \left[\cos \theta_{M+1} \cos \theta_{N+1} e^{-i t\left(\lambda_{N+1,+}-\lambda_{M+1,+}\right)} \tilde{\rho}_{N+1, M+1}^{++,}(4.21)\right.\right. \\
&-\sin \theta_{M+1} \cos \theta_{N+1} e^{-i t\left(\lambda_{N+1,+}-\lambda_{M+1,-}\right)} \tilde{\rho}_{N+1, M+1}^{+,-} \\
&\left.-\sin \theta_{N+1} \cos \theta_{M+1} e^{-i t\left(\lambda_{N+1,-}-\lambda_{M+1,+}\right.}\right)\left(\tilde{\rho}_{M+1, N+1}^{+,-}\right)^{*} \\
&\left.+\sin \theta_{M+1} \sin \theta_{N+1} e^{-i t\left(\lambda_{N+1,-}-\lambda_{M+1,-}\right)} \tilde{\rho}_{N+1, M+1}^{-,-}\right]-\left(\sin ^{2} \theta_{M}+\sin ^{2} \theta_{N}\right) \tilde{\rho}_{N M}^{-,-} \\
&\left.\quad+\frac{1}{2}\left(\sin 2 \theta_{N} e^{-i t\left(\lambda_{N,+}-\lambda_{N,-}\right)} \tilde{\rho}_{N M}^{+,-}+\sin 2 \theta_{M} e^{i t\left(\lambda_{M,+}-\lambda_{M,-}\right)}\left(\tilde{\rho}_{M N}^{+,-}\right)^{*}\right)\right\} ; \\
& \frac{d}{d t} \tilde{\rho}_{N, M}^{+,-}=\frac{\gamma}{2}\left\{2 \operatorname { s i n } \theta _ { N } \operatorname { c o s } \theta _ { M } e ^ { i t ( \lambda _ { N , + } - \lambda _ { M , - } ) } \left[e^{i t\left(\lambda_{M+1,+}-\lambda_{N+1,+}\right)} \cos \theta_{M+1} \cos \theta_{N+1} \tilde{\rho}_{N+1, M+1}^{+,+}\right.\right. \\
&-\quad e^{-i t\left(\lambda_{N+1,+}-\lambda_{M+1,-}\right)} \sin \theta_{M+1} \cos \theta_{N+1} \tilde{\rho}_{N+1, M+1}^{+,-} \\
&- e^{i t\left(\lambda_{M+1,+}-\lambda_{N+1,-}\right)} \cos \theta_{M+1} \sin \theta_{N+1}\left(\tilde{\rho}_{M+1, N+1}^{+,-}\right)^{*} \\
&+\left.e^{-i t\left(\lambda_{N+1,-}-\lambda_{M+1,-}\right)} \sin \theta_{M+1} \sin \theta_{N+1} \tilde{\rho}_{N+1, M+1}^{-,-}\right] \\
&\left.-\left(\cos ^{2} \theta_{N}+\sin ^{2} \theta_{M}\right) \tilde{\rho}_{N M}^{+,-}+\frac{1}{2}\left(\sin 2 \theta_{N} e^{i t\left(\lambda_{N,+}-\lambda_{N,-}\right)} \tilde{\rho}_{N M}^{-,-}+\sin 2 \theta_{M} e^{i t\left(\lambda_{M,+}-\lambda_{M,-}\right)} \tilde{\rho}_{N M}^{+,+}\right)\right\} .
\end{aligned}
$$

Continuando com as simplificações e repetindo o procedimento anterior para separar as equações diferenciais, a defasagem atômica pura terá as seguintes equações

$$
\begin{aligned}
\frac{d}{d t} \tilde{\rho}_{N, M}^{+,+}= & \frac{\gamma_{\phi}}{2}\left[\left(\cos 2 \theta_{M} \cos 2 \theta_{N}-1\right) \tilde{\rho}_{N M}^{+,+}-e^{-i t\left(\lambda_{M,+}-\lambda_{M,-}\right)} \sin 2 \theta_{M} \cos 2 \theta_{N} \tilde{\rho}_{N M}^{+,-}\right. \\
& \left.-\cos 2 \theta_{M} e^{i t\left(\lambda_{N,+}-\lambda_{N,-}\right)} \sin 2 \theta_{N}\left(\tilde{\rho}_{M N}^{+,-}\right)^{*}+e^{i t\left(\lambda_{N,+}-\lambda_{N,-}\right)} e^{-i t\left(\lambda_{M,+}-\lambda_{M,-}\right)} \sin 2 \theta_{M} \sin 2 \theta_{N} \tilde{\rho}_{N M}^{-,-}\right] \\
\frac{d}{d t} \tilde{\rho}_{N M}^{-,-}= & \frac{\gamma_{\phi}}{2}\left[\left(\cos 2 \theta_{M} \cos 2 \theta_{N}-1\right) \tilde{\rho}_{N M}^{-,-}+e^{i t\left(\lambda_{M,+}-\lambda_{M,-}\right)} \sin 2 \theta_{M} \cos 2 \theta_{N}\left(\tilde{\rho}_{M N}^{+,-}\right)^{*}\right. \\
& \left.+\cos 2 \theta_{M} e^{-i t\left(\lambda_{N,+}-\lambda_{N,-}\right)} \sin 2 \theta_{N} \tilde{\rho}_{N M}^{+,-}+e^{i t\left(\lambda_{M,+}-\lambda_{M,-}\right)} \sin 2 \theta_{M} e^{-i t\left(\lambda_{N,+}-\lambda_{N,-}\right)} \sin 2 \theta_{N} \tilde{\rho}_{N M}^{+,+}\right] \\
\frac{d}{d t} \tilde{\rho}_{N, M}^{+,-}= & -\frac{\gamma_{\phi}}{2}\left[\left(\cos 2 \theta_{M} \cos 2 \theta_{N}+1\right) \tilde{\rho}_{N M}^{+,-}+e^{i t\left(\lambda_{M,+}-\lambda_{M,-}\right)} \sin 2 \theta_{M} \cos 2 \theta_{N} \tilde{\rho}_{N M}^{+,+}\right. \\
& \left.-\cos 2 \theta_{M} e^{i t\left(\lambda_{N,+}-\lambda_{N,-}\right)} \sin 2 \theta_{N} \tilde{\rho}_{N M}^{-,-}-e^{i t\left(\lambda_{N,+}-\lambda_{N,-}\right)} e^{i t\left(\lambda_{M,+}-\lambda_{M,-}\right)} \sin 2 \theta_{M} \sin 2 \theta_{N}\left(\tilde{\rho}_{M N}^{+,-}\right)^{*}\right]
\end{aligned}
$$


Por fim, no caso da relaxação pura da cavidade

$$
\begin{aligned}
& \frac{d}{d t} \tilde{\rho}_{N M}^{+,+}=\frac{\kappa}{2}\left\{2 e^{i t\left(\lambda_{N,+}-\lambda_{M,+}\right)}\left[e^{i t\left(\lambda_{M+1,+}-\lambda_{N+1,+}\right.}\right)\left(\sqrt{M+1} \sin \theta_{M} \sin \theta_{M+1}+\sqrt{M} \cos \theta_{M} \cos \theta_{M+1}\right)\right. \\
& \times\left(\sqrt{N+1} \sin \theta_{N} \sin \theta_{N+1}+\sqrt{N} \cos \theta_{N} \cos \theta_{N+1}\right) \tilde{\rho}_{N+1, M+1}^{+,+} \\
& +e^{-i t\left(\lambda_{N+1,+}-\lambda_{M+1,-}\right)}\left(\sqrt{M+1} \sin \theta_{M} \cos \theta_{M+1}-\sqrt{M} \cos \theta_{M} \sin \theta_{M+1}\right) \\
& \times\left(\sqrt{N+1} \sin \theta_{N} \sin \theta_{N+1}+\sqrt{N} \cos \theta_{N} \cos \theta_{N+1}\right) \tilde{\rho}_{N+1, M+1}^{+,-} \\
& +e^{i t\left(\lambda_{M+1,+}-\lambda_{N+1,-}\right)}\left(\sqrt{M+1} \sin \theta_{M} \sin \theta_{M+1}+\sqrt{M} \cos \theta_{M} \cos \theta_{M+1}\right) \\
& \times\left(\sqrt{N+1} \sin \theta_{N} \cos \theta_{N+1}-\sqrt{N} \cos \theta_{N} \sin \theta_{N+1}\right)\left(\tilde{\rho}_{M+1, N+1}^{+,-}\right)^{*} \\
& +e^{i t\left(\lambda_{M+1,-}-\lambda_{N+1,-}\right)}\left(\sqrt{M+1} \sin \theta_{M} \cos \theta_{M+1}-\sqrt{M} \cos \theta_{M} \sin \theta_{M+1}\right) \\
& \left.\times\left(\sqrt{N+1} \sin \theta_{N} \cos \theta_{N+1}-\sqrt{N} \cos \theta_{N} \sin \theta_{N+1}\right) \tilde{\rho}_{N+1, M+1}^{-,-}\right] \\
& -\left[N+M-\cos ^{2} \theta_{N}-\cos ^{2} \theta_{M}\right] \tilde{\rho}_{N M}^{+,+} \\
& \left.-\frac{1}{2}\left[e^{-i t\left(\lambda_{M,+}-\lambda_{M,-}\right)} \sin 2 \theta_{M} \tilde{\rho}_{N M}^{+,-}+e^{i t\left(\lambda_{N,+}-\lambda_{N,-}\right)} \sin 2 \theta_{N}\left(\tilde{\rho}_{M N}^{+,-}\right)^{*}\right]\right\} ; \\
& \frac{d}{d t} \tilde{\rho}_{N M}^{-,-}=\frac{\kappa}{2}\left\{2 e^{i t\left(\lambda_{N,-}-\lambda_{M,-}\right)}\left[e^{i t\left(\lambda_{M+1,+}-\lambda_{N+1,+}\right.}\right)\left(\sqrt{M+1} \cos \theta_{M} \sin \theta_{M+1}-\sqrt{M} \sin \theta_{M} \cos \theta_{M+1}\right)\right. \\
& \times\left(\sqrt{N+1} \cos \theta_{N} \sin \theta_{N+1}-\sqrt{N} \sin \theta_{N} \cos \theta_{N+1}\right) \tilde{\rho}_{N+1, M+1}^{+,+} \\
& +e^{-i t\left(\lambda_{N+1,+}-\lambda_{M+1,-}\right)}\left(\sqrt{M+1} \cos \theta_{M} \cos \theta_{M+1}+\sqrt{M} \sin \theta_{M} \sin \theta_{M+1}\right) \\
& \times\left(\sqrt{N+1} \cos \theta_{N} \sin \theta_{N+1}-\sqrt{N} \sin \theta_{N} \cos \theta_{N+1}\right) \tilde{\rho}_{N+1, M+1}^{+,-} \\
& +e^{i t\left(\lambda_{M+1,+}-\lambda_{N+1,-}\right)}\left(\sqrt{M+1} \cos \theta_{M} \sin \theta_{M+1}-\sqrt{M} \sin \theta_{M} \cos \theta_{M+1}\right) \\
& \times\left(\sqrt{N+1} \cos \theta_{N} \cos \theta_{N+1}+\sqrt{N} \sin \theta_{N} \sin \theta_{N+1}\right)\left(\tilde{\rho}_{M+1, N+1}^{+,-}\right)^{*} \\
& +e^{i t\left(\lambda_{M+1,-}-\lambda_{N+1,-}\right)}\left(\sqrt{M+1} \cos \theta_{M} \cos \theta_{M+1}+\sqrt{M} \sin \theta_{M} \sin \theta_{M+1}\right) \\
& \left.\times\left(\sqrt{N+1} \cos \theta_{N} \cos \theta_{N+1}+\sqrt{N} \sin \theta_{N} \sin \theta_{N+1}\right) \tilde{\rho}_{N+1, M+1}^{-,-}\right] \\
& -\left[N+M-\sin ^{2} \theta_{N}-\sin ^{2} \theta_{M}\right] \tilde{\rho}_{N M}^{-,-} \\
& \left.-\frac{1}{2}\left[e^{-i t\left(\lambda_{N,+}-\lambda_{N,-}\right)} \sin 2 \theta_{N} \tilde{\rho}_{N M}^{+,-}+e^{i t\left(\lambda_{M,+}-\lambda_{M,-}\right)} \sin 2 \theta_{M}\left(\tilde{\rho}_{M N}^{+,-}\right)^{*}\right]\right\} ;
\end{aligned}
$$




$$
\begin{aligned}
\frac{d}{d t} \tilde{\rho}_{N M}^{+,-}= & \frac{\kappa}{2}\left\{2 e^{i t\left(\lambda_{N,+}-\lambda_{M,-}\right)}\left[e^{i t\left(\lambda_{M+1,+}-\lambda_{N+1,+}\right.}\right)\left(\sqrt{M+1} \cos \theta_{M} \sin \theta_{M+1}-\sqrt{M} \sin \theta_{M} \cos \theta_{M+1}\right)\right. \\
& \times\left(\sqrt{N+1} \sin \theta_{N} \sin \theta_{N+1}+\sqrt{N} \cos \theta_{N} \cos \theta_{N+1}\right) \tilde{\rho}_{N+1, M+1}^{+,+} \\
& +e^{-i t\left(\lambda_{N+1,+}-\lambda_{M+1,-}\right)}\left(\sqrt{M+1} \cos \theta_{M} \cos \theta_{M+1}+\sqrt{M} \sin \theta_{M} \sin \theta_{M+1}\right) \\
& \times\left(\sqrt{N+1} \sin \theta_{N} \sin \theta_{N+1}+\sqrt{N} \cos \theta_{N} \cos \theta_{N+1}\right) \tilde{\rho}_{N+1, M+1}^{+,-} \\
& +e^{i t\left(\lambda_{M+1,+}-\lambda_{N+1,-}\right)}\left(\sqrt{M+1} \cos \theta_{M} \sin \theta_{M+1}-\sqrt{M} \sin \theta_{M} \cos \theta_{M+1}\right) \\
& \times\left(\sqrt{N+1} \sin \theta_{N} \cos \theta_{N+1}-\sqrt{N} \cos \theta_{N} \sin \theta_{N+1}\right)\left(\tilde{\rho}_{M+1, N+1}^{+,-}\right)^{*} \\
& +e^{-i t\left(\lambda_{N+1,-}-\lambda_{M+1,-}\right)}\left(\sqrt{M+1} \cos \theta_{M} \cos \theta_{M+1}+\sqrt{M} \sin \theta_{M} \sin \theta_{M+1}\right) \\
& \left.\times\left(\sqrt{N+1} \sin \theta_{N} \cos \theta_{N+1}-\sqrt{N} \cos \theta_{N} \sin \theta_{N+1}\right) \tilde{\rho}_{N+1, M+1}^{-,-}\right] \\
& -\left[N+M-\cos ^{2} \theta_{N}-\sin ^{2} \theta_{M}\right] \tilde{\rho}_{N M}^{+,-} \\
& \left.-\frac{1}{2}\left[e^{i t\left(\lambda_{N,+}-\lambda_{N,-}\right)} \sin 2 \theta_{N} \tilde{\rho}_{N M}^{-,-}+e^{i t\left(\lambda_{M,+}-\lambda_{M,-}\right)} \sin 2 \theta_{M} \tilde{\rho}_{N M}^{+,+}\right]\right\}
\end{aligned}
$$

O método RWA se mostra muito útil novamente, pois permitirá eliminar termos que oscilam rapidamente nas equações diferenciais para as amplitudes de probabilidade. Quando se considera o par de equações

$$
\frac{d A(t)}{d t}=-q e^{i t w} B(t), \frac{d B(t)}{d t}=-q e^{-i t w} A(t)
$$

as soluções exatas são

$$
A(t)=e^{i t W / 2} \frac{-\left[(W-R) A_{0}-2 i q B_{0}\right] e^{i t R / 2}+\left[(W+R) A_{0}-2 i q B_{0}\right] e^{-i t R / 2}}{2 R}
$$

$\mathrm{e}$

$$
B(t)=e^{-i t W / 2} \frac{\left[(W+R) B_{0}+2 i q A_{0}\right] e^{i R t / 2}-\left[(W-R) B_{0}+2 i q A_{0}\right] e^{-i R t / 2}}{2 R},
$$

onde os parâmetros $q$ e $W$ são constantes, $R=\sqrt{W^{2}-4 q^{2}}$. Para $|q / W| \ll 1$, os resultados aproximados serão $A \simeq A_{0}$ e $B \simeq B_{0}$, ou seja, $d A / d t \simeq 0$ e $d B / d t \simeq 0$ nas equações diferenciais. Então, os shifts de frequência negligenciados serão da ordem de $\mathcal{O}\left(q^{2} / W\right)$. Para uma aplicação direta, no caso $\lambda_{n,+}-\lambda_{n,-}=\beta_{n} \gg \kappa, \gamma, \gamma_{\phi}$ pode-se eliminar termos que oscilarão rapidamente. As frequências ressonantes serão modificadas por causa dos shifts de ressonância desprezados. Assim, as frequências ressonantes deverão ser ajustadas manualmente. 


\section{Capítulo 5}

\section{Comportamento assintótico}

O Hamiltoniano (2.17), como visto no capítulo 2, torna possível analisar a dinâmica unitária para determinados efeitos físicos de maneira mais simplificada. Apesar da dinâmica unitária ter sido analisada em [42] e [43], é necessário que a notação seja compactada para que seja possível trabalhar algebricamente a EMP. Além disso, como a dinâmica foi obtida utilizando-se a representação vestida da interação átomo-campo, no capítulo anterior expandimos o operador densidade na representação dos EV.

Neste capítulo, investigaremos como a dinâmica do sistema será afetada pelos efeitos dissipativos. No entanto, a investigação será restrita ao comportamento analítico assintótico para a Equação Mestra Padrão. Para tanto, o formalismo do capítulo anterior será levado em consideração e o método RWA será muito importante na análise. Os efeitos físicos analisados serão: geração de pares de excitações no Regime Ressonante, Regime anti-Jaynes-Cummings, Regime anti-efeito Casimir dinâmico e efeito Casimir dinâmico fundamental. Além disso, verificaremos como os processos de criação e aniquilação de excitações incrementados (através de modulações multi-frequências) têm suas dinâmicas afetadas pela dissipação. 


\subsection{Comportamento para duas excitações}

\subsubsection{Geração de pares de excitações no regime ressonante}

O objetivo desta seção é demonstrar como a dissipação modifica a dinâmica do processo de criação e aniquilação de fótons a partir do Estado de zero excitações (EZE) $|g, 0\rangle$. Os autovalores (4.2) permitem estipular a frequência ressonante de modulção $\eta^{(j)}$. Usando o resultado obtido (2.17), a modulação em $\eta^{(r)}=2 \omega_{0}+\mathcal{R} g_{0} \sqrt{2}$, com $\mathcal{R}= \pm$, no regime ressonante $\Delta_{-}=0$, fornece a seguinte condição de ressonância: $\lambda_{m+2, \mathcal{S}}-\lambda_{m, \mathcal{T}}=\lambda_{2, \mathcal{R}}-\lambda_{0,-}$

$$
H_{e f f}=\sum_{m, \mathcal{S}, \mathcal{T}} \Xi_{m, \mathcal{T}, \mathcal{S}}^{(r)} e^{-i t\left(\lambda_{m+2, \mathcal{S}}-\lambda_{m, \mathcal{T}}-\eta^{(r)}\right)}\left|\varphi_{m, \mathcal{T}}\right\rangle\left\langle\varphi_{m+2, \mathcal{S}}\right|+\text { h.c. }
$$

Logo, $m=0, \mathcal{S}=\mathcal{R}$ e $\mathcal{T}=-$, daí conclui-se que o Hamiltoniano efetivo será

$$
H_{\text {eff }}=\Xi_{0,-, \mathcal{R}}^{(r)}\left|\varphi_{0,-}\right\rangle\left\langle\varphi_{2, \mathcal{R}}\right|+\text { h.c. }=\theta\left|\varphi_{0,-}\right\rangle\left\langle\varphi_{2, \mathcal{R}}\left|+\theta^{*}\right| \varphi_{2, \mathcal{R}}\right\rangle\left\langle\varphi_{0,-}\right|,
$$

que acopla os estados $\left|\varphi_{0,-}\right\rangle \leftrightarrow\left\langle\varphi_{2, \mathcal{R}}\right|$. Ademais, de acordo com (2.13),

$$
\theta=i g_{0} \mathcal{R} \frac{\sqrt{2}}{4} \Upsilon^{(r)}=i g_{0} \mathcal{R} \frac{\sqrt{2}}{4}\left(\frac{\varepsilon_{\omega}^{(r)}}{2 \omega_{0}}+\frac{\varepsilon_{\Omega}^{(r)}}{2 \omega_{0}}-\frac{\varepsilon_{g}^{(r)}}{g_{0}}+\mathcal{R} i \frac{\sqrt{2} \varepsilon_{\chi}^{(r)}}{g_{0}}\right),
$$

onde $\Upsilon^{(j)}$ é a profundidade total da modulação. O processo consiste, então, na transição periódica entre EZE e estados emaranhados átomo-campo contendo duas excitações no total.

A dinâmica unitária é dada pela equação

$$
\frac{d \tilde{\rho}}{d t}=-i\left[H_{e f f}, \tilde{\rho}\right]
$$

que está na representação de interação. Lembrando que $\tilde{\rho}$ é escrito como

$$
\begin{aligned}
\tilde{\rho} & =\sum_{\mathcal{S}, \mathcal{T}} \sum_{N, M=0}^{\infty} \tilde{\rho}_{N, M}^{\mathcal{S}, \mathcal{T}}\left|\varphi_{N, \mathcal{S}}\right\rangle\left\langle\varphi_{M, \mathcal{T}}\right| \\
& =\sum_{N, M=0}^{\infty}\left\{\tilde{\rho}_{N, M}^{+,+}\left|\varphi_{N,+}\right\rangle\left\langle\varphi_{M,+}\left|+\tilde{\rho}_{N, M}^{-,-}\right| \varphi_{N,-}\right\rangle\left\langle\varphi_{M,-}\left|+\tilde{\rho}_{N, M}^{+,-}\right| \varphi_{N,+}\right\rangle\left\langle\varphi_{M,-}\left|+\left(\tilde{\rho}_{M, N}^{+,-}\right)^{*}\right| \varphi_{N,-}\right\rangle\left\langle\varphi_{M,+}\right|\right\},
\end{aligned}
$$

a equação para a dinâmica se simplifica à forma

$$
\begin{aligned}
\frac{d}{d t} \tilde{\rho}_{N, M}^{\mathcal{S}, \mathcal{T}}= & -i\left\langle\varphi_{N, \mathcal{S}}\right|\left[\left(\sum_{\mathcal{S}, \mathcal{T}} \sum_{N, M=0}^{\infty} \tilde{\rho}_{N, M}^{\mathcal{S}, \mathcal{T}}\left(\theta\left|\varphi_{0,-}\right\rangle\left\langle\varphi_{2, \mathcal{R}}|| \varphi_{N, \mathcal{S}}\right\rangle\left\langle\varphi_{M, \mathcal{T}}\left|+\theta^{*}\right| \varphi_{2, \mathcal{R}}\right\rangle\left\langle\varphi_{0,-}|| \varphi_{N, \mathcal{S}}\right\rangle\left\langle\varphi_{M, \mathcal{T}}\right|\right)\right)\right. \\
& \left.-\left(\sum_{\mathcal{S}, \mathcal{T}} \sum_{N, M=0}^{\infty} \tilde{\rho}_{N, M}^{\mathcal{S}, \mathcal{T}}\left(\theta\left|\varphi_{N, \mathcal{S}}\right\rangle\left\langle\varphi_{M, \mathcal{T}}|| \varphi_{0,-}\right\rangle\left\langle\varphi_{2, \mathcal{R}}\left|+\theta^{*}\right| \varphi_{N, \mathcal{S}}\right\rangle\left\langle\varphi_{M, \mathcal{T}}|| \varphi_{2, \mathcal{R}}\right\rangle\left\langle\varphi_{0,-}\right|\right)\right)\right]\left|\varphi_{M, \mathcal{T}}\right\rangle \\
= & -i\left[\left(\delta_{N, 0} \delta_{\mathcal{S},-} \theta \tilde{\rho}_{2, M}^{\mathcal{R}, \mathcal{T}}+\delta_{N, 2} \delta_{\mathcal{S}, \mathcal{R}} \theta^{*} \tilde{\rho}_{0, M}^{-, \mathcal{T}}\right)-\left(\delta_{M, 2} \delta_{\mathcal{R}, \mathcal{T}} \theta \tilde{\rho}_{N,-0}^{\mathcal{S},-}+\delta_{M, 0} \delta_{\mathcal{T},-} \theta^{*} \tilde{\rho}_{N, 2}^{\mathcal{S}, \mathcal{R}}\right)\right]
\end{aligned}
$$


Como o estado inicial é $\left|\varphi_{0,-}\right\rangle=|g, 0\rangle$, é apropriado escrever a equação diferencial para $\tilde{\rho}_{0,0}^{-,-}$, que é a população para o estado $|g, 0\rangle$. Como é possível observar aparecerão os termos $\left(\theta \tilde{\rho}_{2,0}^{\mathcal{R},-}\right)^{i}$ e $\tilde{\rho}_{2,2}^{\mathcal{R}, \mathcal{R}}$ (o índice sobrescrito $i$ em $\left(\theta \tilde{\rho}_{2,0}^{\mathcal{R},-}\right)^{i}$ indica que está sendo tomada somente a parte imaginária do termo), fazendo-se necessário escrever a equação diferencial para estes termos:

$$
\begin{aligned}
\frac{d}{d t} \tilde{\rho}_{0,0}^{-,-} & =2\left(\theta \tilde{\rho}_{2,0}^{\mathcal{R},-}\right)^{i} ; \\
\frac{d}{d t}\left(\theta \tilde{\rho}_{2,0}^{\mathcal{R},-}\right)^{i} & =-|\theta|^{2}\left[\tilde{\rho}_{0,0}^{-,-}-\tilde{\rho}_{2,2}^{\mathcal{R}, \mathcal{R}}\right] ; \\
\frac{d}{d t} \tilde{\rho}_{2,2}^{\mathcal{R}, \mathcal{R}} & =-2\left(\theta \tilde{\rho}_{2,0}^{\mathcal{R},-}\right)^{i} .
\end{aligned}
$$

Agora o comportamento assintótico do sistema será analisado quando a dissipação está presente. Usando as equações (4.20), (4.21) e (4.22) para relaxação atômica pura, uma formulação geral será escrita (lembrando que no RR: $\theta_{0}=0$ e $\theta_{n>0}=\pi / 4$ )

$$
\begin{aligned}
\frac{d}{d t} \tilde{\rho}_{0,0}^{-,-}= & \frac{\gamma}{2}\left(\tilde{\rho}_{1,1}^{+,+}+\tilde{\rho}_{1,1}^{-,-}\right), \\
\frac{d}{d t} \tilde{\rho}_{0, M>0}^{-,-}= & -\frac{1}{2} \frac{\gamma}{2} \tilde{\rho}_{0, M}^{-,-}, \\
\frac{d}{d t} \tilde{\rho}_{N>0,0}^{-,-}= & -\frac{1}{2} \frac{\gamma}{2} \tilde{\rho}_{N, 0}^{-,-}, \\
\frac{d}{d t} \tilde{\rho}_{N>0, M>0}^{-,-}= & \frac{\gamma}{2}\left(\frac { 1 } { 2 } e ^ { i t ( \lambda _ { N , - } - \lambda _ { M , - } ) } \left(e^{-i t\left(\lambda_{N+1,+}-\lambda_{M+1,+}\right)} \tilde{\rho}_{N+1, M+1}^{+,+}\right.\right. \\
& \left.\left.+e^{-i t\left(\lambda_{N+1,-}-\lambda_{M+1,-}\right)} \tilde{\rho}_{N+1, M+1}^{-,-}\right)-\tilde{\rho}_{N M}^{-,-}\right), \\
\frac{d}{d t} \tilde{\rho}_{N>0, M \neq N, M>0}^{+,+}= & -\frac{\gamma}{2} \tilde{\rho}_{N M}^{+,+}, \\
\frac{d}{d t} \tilde{\rho}_{N>0, N}^{+,+}= & \frac{\gamma}{2}\left(\frac{1}{2}\left[\tilde{\rho}_{N+1, M+1}^{+,+}+\tilde{\rho}_{N+1, M+1}^{-,-}\right]-\tilde{\rho}_{N M}^{+,+}\right), \\
\frac{d}{d t} \tilde{\rho}_{N>0,0}^{+,-}= & -\frac{1}{2} \frac{\gamma}{2} \tilde{\rho}_{N, 0}^{+,-}, \\
\frac{d}{d t} \tilde{\rho}_{N>0, M>0}^{+,-}= & \frac{\gamma}{2}\left(\frac { 1 } { 2 } e ^ { i t ( \lambda _ { N , + } - \lambda _ { M , - } ) } \left(e^{i t\left(\lambda_{M+1,+}-\lambda_{N+1,+}\right)} \tilde{\rho}_{N+1, M+1}^{+,+}\right.\right. \\
& \left.\left.+e^{-i t\left(\lambda_{N+1,-}-\lambda_{M+1,-}\right)} \tilde{\rho}_{N+1, M+1}^{-,-}\right)-\tilde{\rho}_{N M}^{+,-}\right) .
\end{aligned}
$$

A equação para a população $\tilde{\rho}_{0,0}^{-,-}$sob relaxação atômica pura envolve termos $\tilde{\rho}_{1,1}^{\mathcal{R}, \mathcal{R}}(\operatorname{com} \mathcal{R}= \pm$ ), de modo que suas equações precisam ser escritas. No geral, pela estrutura das equações, é possível notar que populações são acopladas entre si. Nesta seção serão consideradas no máximo duas excitações (termos com $N>2$ serão negligenciados) e o método RWA será de grande utilidade, visto que 
haverão termos que oscilam rapidamente e serão desprezados. As equações para a evolução unitária sob a relaxação atômica pura são

$$
\begin{gathered}
\frac{d}{d t} \tilde{\rho}_{0,0}^{-,-}=2\left(\theta \tilde{\rho}_{2,0}^{\mathcal{R},-}\right)^{i}+\frac{\gamma}{2}\left(\tilde{\rho}_{1,1}^{\mathcal{R}, \mathcal{R}}+\tilde{\rho}_{1,1}^{-\mathcal{R},-\mathcal{R}}\right), \\
\frac{d}{d t}\left(\theta \tilde{\rho}_{2,0}^{\mathcal{R},-}\right)^{i}=-|\theta|^{2}\left[\tilde{\rho}_{0,0}^{-,-}-\tilde{\rho}_{2,2}^{\mathcal{R}, \mathcal{R}}\right]-\frac{1}{2} \frac{\gamma}{2}\left(\theta \tilde{\rho}_{2,0}^{\mathcal{R},-}\right)^{i}, \\
\frac{d}{d t} \tilde{\rho}_{1,1}^{\mathcal{R}, \mathcal{R}}=\frac{\gamma}{2}\left(\frac{1}{2}\left[\tilde{\rho}_{2,2}^{\mathcal{R}, \mathcal{R}}+\tilde{\rho}_{2,2}^{-\mathcal{R},-\mathcal{R}}\right]-\tilde{\rho}_{11}^{\mathcal{R}, \mathcal{R}}\right), \\
\frac{d}{d t} \tilde{\rho}_{1,1}^{-\mathcal{R},-\mathcal{R}}=\frac{\gamma}{2}\left(\frac{1}{2}\left[\tilde{\rho}_{2,2}^{-\mathcal{R},-\mathcal{R}}+\tilde{\rho}_{2,2}^{\mathcal{R}, \mathcal{R}}\right]-\tilde{\rho}_{11}^{-\mathcal{R},-\mathcal{R}}\right), \\
\frac{d}{d t} \tilde{\rho}_{2,2}^{\mathcal{R}, \mathcal{R}}=-2\left(\theta \tilde{\rho}_{2,0}^{\mathcal{R},-}\right)^{i}-\frac{\gamma}{2} \tilde{\rho}_{22}^{\mathcal{R}, \mathcal{R}} \\
\frac{d}{d t} \tilde{\rho}_{2,2}^{-\mathcal{R},-\mathcal{R}}=-\frac{\gamma}{2} \tilde{\rho}_{22}^{-\mathcal{R},-\mathcal{R}},
\end{gathered}
$$

sendo que a aproximação $\lambda_{n,+}-\lambda_{n,-}=\beta_{n} \gg \gamma$ permitiu eliminar termos que oscilam rapidamente nas exponenciais através do método RWA. A solução assintótica é uma análise interessante, pois permite saber quais populações serão permitidas após o sistema evoluir por um tempo grande. Entretanto, o preço que se paga é não saber como o sistema se comporta ao longo do tempo. Fazendo o lado esquerdo das equações igual a zero, a solução assintótica será

$$
\begin{gathered}
\tilde{\rho}_{22}^{-\mathcal{R},-\mathcal{R}}=0, \\
\tilde{\rho}_{11}^{\mathcal{R}, \mathcal{R}}=\frac{1}{2} \tilde{\rho}_{2,2}^{\mathcal{R}, \mathcal{R}}, \\
\tilde{\rho}_{11}^{-\mathcal{R},-\mathcal{R}}=\frac{1}{2} \tilde{\rho}_{2,2}^{\mathcal{R}, \mathcal{R}}, \\
\left(\theta \tilde{\rho}_{2,0}^{\mathcal{R},-}\right)^{i}=\frac{-\gamma}{2} \tilde{\rho}_{1,1}^{\mathcal{R}, \mathcal{R}}, \\
\tilde{\rho}_{2,2}^{\mathcal{R}, \mathcal{R}}=\frac{|\theta|^{2}}{\left(|\theta|^{2}+(\gamma / 4)^{2}\right)} \tilde{\rho}_{0,0}^{-,-},
\end{gathered}
$$

que devido à normalização

$$
\tilde{\rho}_{0,0}^{-,-}+\tilde{\rho}_{11}^{\mathcal{R}, \mathcal{R}}+\tilde{\rho}_{11}^{-\mathcal{R},-\mathcal{R}}+\tilde{\rho}_{2,2}^{\mathcal{R}, \mathcal{R}}+\tilde{\rho}_{22}^{-\mathcal{R},-\mathcal{R}}=1
$$

torna possível escrever as probabilidades

$$
\tilde{\rho}_{0,0}^{-,-}=\frac{|\theta|^{2}+(\gamma / 4)^{2}}{3|\theta|^{2}+(\gamma / 4)^{2}}, \tilde{\rho}_{2,2}^{\mathcal{R}, \mathcal{R}}=\frac{|\theta|^{2}}{3|\theta|^{2}+(\gamma / 4)^{2}}, \tilde{\rho}_{11}^{\mathcal{R}, \mathcal{R}}=\tilde{\rho}_{11}^{-\mathcal{R},-\mathcal{R}}=\frac{1}{2} \frac{|\theta|^{2}}{3|\theta|^{2}+(\gamma / 4)^{2}} .
$$


Fisicamente, o que se deseja é que os efeitos de dissipação sejam minimizados. Diante disso, a condição desejada é $|\theta| \gg \gamma$ fornecendo as probabilidades para os estados

$$
\tilde{\rho}_{0,0}^{-,-} \approx \frac{1}{3}, \tilde{\rho}_{2,2}^{\mathcal{R}, \mathcal{R}} \approx \frac{1}{3}, \tilde{\rho}_{11}^{\mathcal{R}, \mathcal{R}} \approx \tilde{\rho}_{11}^{-\mathcal{R},-\mathcal{R}} \approx \frac{1}{6}
$$

Como observado, duas novas populações $\tilde{\rho}_{11}^{\mathcal{R}, \mathcal{R}}$ e $\tilde{\rho}_{11}^{-\mathcal{R},-\mathcal{R}}$ surgem mostrando que a relaxação atômica altera a dinâmica unitária.

No caso da defasagem atômica pura, as equações (4.23), (4.24) e (4.25) serão necessárias. Após novamente ter sido utilizado o método RWA, aproximando $\lambda_{n,+}-\lambda_{n,-}=\beta_{n} \gg \gamma_{\phi}$, termos que oscilam rapidamente foram eliminados para que se pudesse chegar a dinâmica sob DAP

$$
\begin{gathered}
\frac{d}{d t} \tilde{\rho}_{00}^{-,-}=2\left(\theta \tilde{\rho}_{2,0}^{\mathcal{R},-}\right)^{i} \\
\frac{d}{d t}\left(\theta \tilde{\rho}_{2,0}^{\mathcal{R},-}\right)^{i}=-|\theta|^{2}\left[\tilde{\rho}_{0,0}^{-,-}-\tilde{\rho}_{2,2}^{\mathcal{R}, \mathcal{R}}\right]-\frac{\gamma_{\phi}}{2}\left(\theta \tilde{\rho}_{20}^{\mathcal{R},-}\right)^{i} \\
\frac{d}{d t} \tilde{\rho}_{2,2}^{\mathcal{R}, \mathcal{R}}=-2\left(\theta \tilde{\rho}_{2,0}^{\mathcal{R},-}\right)^{i}+\frac{\gamma_{\phi}}{2}\left[-\tilde{\rho}_{22}^{\mathcal{R}, \mathcal{R}}+\tilde{\rho}_{22}^{-\mathcal{R},-\mathcal{R}}\right] \\
\frac{d}{d t} \tilde{\rho}_{2,2}^{-\mathcal{R},-\mathcal{R}}=\frac{\gamma_{\phi}}{2}\left[-\tilde{\rho}_{22}^{-\mathcal{R},-\mathcal{R}}+\tilde{\rho}_{22}^{\mathcal{R}, \mathcal{R}}\right]
\end{gathered}
$$

com soluções assintóticas (e sob a condição de normalização)

$$
\tilde{\rho}_{0,0}^{-,-}=\tilde{\rho}_{22}^{-\mathcal{R},-\mathcal{R}}=\tilde{\rho}_{2,2}^{\mathcal{R}, \mathcal{R}}=\frac{1}{3} .
$$

Esse resultado é interessante, pois mostra que a dinâmica unitária é afetada pela defasagem atômica. O termo não-diagonal $\left(\theta \tilde{\rho}_{2,0}^{\mathcal{R},-}\right)^{i}$ vai a zero assintoticamente, enquanto a população $\tilde{\rho}_{22}^{-\mathcal{R},-\mathcal{R}}$ passa a ser não-nula.

Analisando como a dinâmica unitária será afetada pela relaxação pura da cavidade, de posse das equações (4.26), (4.27) e (4.28), obtem-se

$$
\begin{gathered}
\frac{d}{d t} \tilde{\rho}_{00}^{-,-}=2\left(\theta \tilde{\rho}_{2,0}^{\mathcal{R},-}\right)^{i}+\frac{\kappa}{2}\left(\tilde{\rho}_{1,1}^{\mathcal{R}, \mathcal{R}}+\tilde{\rho}_{1,1}^{-\mathcal{R},-\mathcal{R}}\right), \\
\frac{d}{d t}\left(\theta \tilde{\rho}_{2,0}^{\mathcal{R},-}\right)^{i}=-|\theta|^{2}\left[\tilde{\rho}_{0,0}^{-,-}-\tilde{\rho}_{2,2}^{\mathcal{R}, \mathcal{R}}\right]-\frac{3}{4} \kappa\left(\theta \tilde{\rho}_{2,0}^{\mathcal{R},-}\right)^{i}, \\
\frac{d}{d t} \tilde{\rho}_{1,1}^{\mathcal{R}, \mathcal{R}}=\frac{\kappa}{2}\left(\frac{1}{2}\left[(\sqrt{2}+1)^{2} \tilde{\rho}_{2,2}^{\mathcal{R}, \mathcal{R}}+(\sqrt{2}-1)^{2} \tilde{\rho}_{2,2}^{-\mathcal{R},-\mathcal{R}}\right]-\tilde{\rho}_{11}^{\mathcal{R}, \mathcal{R}}\right), \\
\frac{d}{d t} \tilde{\rho}_{1,1}^{-\mathcal{R},-\mathcal{R}}=\frac{\kappa}{2}\left(\frac{1}{2}\left[(\sqrt{2}+1)^{2} \tilde{\rho}_{2,2}^{-\mathcal{R},-\mathcal{R}}+(\sqrt{2}-1)^{2} \tilde{\rho}_{2, \mathcal{R}}^{\mathcal{R}, \mathcal{R}}\right]-\tilde{\rho}_{11}^{-\mathcal{R},-\mathcal{R}}\right),
\end{gathered}
$$




$$
\begin{gathered}
\frac{d}{d t} \tilde{\rho}_{2,2}^{\mathcal{R}, \mathcal{R}}=-2\left(\theta \tilde{\rho}_{2,0}^{\mathcal{R},-}\right)^{i}-\frac{3}{2} \kappa \tilde{\rho}_{22}^{\mathcal{R}, \mathcal{R}}, \\
\frac{d}{d t} \tilde{\rho}_{2,2}^{-\mathcal{R},-\mathcal{R}}=-\frac{3}{2} \kappa \tilde{\rho}_{22}^{-\mathcal{R},-\mathcal{R}} .
\end{gathered}
$$

Lembrando que o método RWA foi importante para chegar a tais equações. Igualando o lado esquerdo das equações a zero o resultado assintótico será

$$
\begin{gathered}
\left(\theta \tilde{\rho}_{2,0}^{\mathcal{R},-}\right)^{i}=-\frac{3}{4} \kappa \tilde{\rho}_{22}^{\mathcal{R}, \mathcal{R}}, \\
\tilde{\rho}_{22}^{-\mathcal{R},-\mathcal{R}}=0, \\
\tilde{\rho}_{22}^{\mathcal{R}, \mathcal{R}}=\frac{|\theta|^{2}}{|\theta|^{2}+(3 \kappa / 4)^{2}} \tilde{\rho}_{0,0}^{-,-}, \\
\tilde{\rho}_{11}^{\mathcal{R}, \mathcal{R}}=\frac{1}{2}(\sqrt{2}+1)^{2} \tilde{\rho}_{2,2}^{\mathcal{R}, \mathcal{R}}, \\
\tilde{\rho}_{11}^{-\mathcal{R},-\mathcal{R}}=\frac{1}{2}(\sqrt{2}-1)^{2} \tilde{\rho}_{2,2}^{\mathcal{R}, \mathcal{R}} .
\end{gathered}
$$

Em virtude da condição de normalização conclui-se que as populações atingidas são

$$
\begin{aligned}
\tilde{\rho}_{0,0}^{-,-} & =\frac{|\theta|^{2}+(3 \kappa / 4)^{2}}{5|\theta|^{2}+(3 \kappa / 4)^{2}}, \tilde{\rho}_{22}^{\mathcal{R}, \mathcal{R}}=\frac{|\theta|^{2}}{5|\theta|^{2}+(3 \kappa / 4)^{2}}, \\
\tilde{\rho}_{11}^{\mathcal{R}, \mathcal{R}} & =\frac{1}{2}(\sqrt{2}+1)^{2} \tilde{\rho}_{2,2}^{\mathcal{R}, \mathcal{R}}, \quad \tilde{\rho}_{11}^{-\mathcal{R},-\mathcal{R}}=\frac{1}{2}(\sqrt{2}-1)^{2} \tilde{\rho}_{2,2}^{\mathcal{R}, \mathcal{R}}
\end{aligned}
$$

com probalidades, na condição $|\theta| \gg \kappa$,

$$
\tilde{\rho}_{0,0}^{-,-} \approx \frac{1}{5}, \tilde{\rho}_{22}^{\mathcal{R}, \mathcal{R}} \approx \frac{1}{5}, \tilde{\rho}_{11}^{\mathcal{R}, \mathcal{R}} \approx 0,58, \tilde{\rho}_{11}^{-\mathcal{R},-\mathcal{R}} \approx 1,71 \times 10^{-2} .
$$

Logo, a dinâmica unitária é drásticamente afetada, já que estados antes não atingidos tornam-se permitidos graças aos efeitos da dissipação. É importante notar que em todos os casos o sistema atinge um estado estacionário com número finito de excitações.

\subsubsection{Regime anti-Jaynes-Cummings (AJC)}

Agora vamos considerar a modulação com frequência ressonante $\eta^{(J)}=\omega_{0}+\Omega_{0}-2\left(\delta_{-}-\delta_{+}\right)$no regime dispersivo $\left|\Delta_{-}\right| / 2 \gg g_{0} \sqrt{n_{\text {máx }}}$ (com $n_{\text {máx }}$ sendo o número máximo de excitações), desprezandose correções de ordens superiores [43], onde $\delta_{ \pm}=g_{0}^{2} / \Delta_{ \pm}\left(\Delta_{+} \equiv \omega_{0}+\Omega_{0}\right)$. Como mostrado em [43], tal 
ressonância ocorre quando $\eta^{(J)} \simeq \lambda_{2,-\mathcal{D}}-\lambda_{0, \mathcal{D}}$. A dinâmica unitária será descrita pelo Hamiltoniano efetivo

$$
H_{e f f}=\sum_{m, \mathcal{S}, \mathcal{T}} \Xi_{m, \mathcal{T}, \mathcal{S}}^{(r)} e^{-i t\left(\lambda_{m+2, \mathcal{S}}-\lambda_{m, \mathcal{T}}-\eta^{(J)}\right)}\left|\varphi_{m, \mathcal{T}}\right\rangle\left\langle\varphi_{m+2, \mathcal{S}}\right|+\text { h.c. }
$$

de modo que a frequência será ressonante se $m=0, \mathcal{S}=-\mathcal{D}$ e $\mathcal{T}=\mathcal{D}$. Logo, obtemos o Hamiltoniano de anti-Jaynes-Cummings [41,49]

$$
H_{A J C}=\Xi_{0, \mathcal{D},-\mathcal{D}}^{(r)}\left|\varphi_{0, \mathcal{D}}\right\rangle\left\langle\varphi_{2,-\mathcal{D}}\right|+\text { h.c. }=\theta\left|\varphi_{0,-}\right\rangle\left\langle\varphi_{2,-\mathcal{D}}\left|+\theta^{*}\right| \varphi_{2,-\mathcal{D}}\right\rangle\left\langle\varphi_{0,-}\right|
$$

com

$$
\begin{gathered}
\theta=i \frac{1}{2} g_{0} \mathcal{D} \Upsilon^{(J)} \\
\Upsilon^{(J)} \equiv\left[-\frac{\varepsilon_{\omega}^{(J)}}{\Delta_{+}}-\frac{\varepsilon_{\Omega}^{(J)}}{\Delta_{+}}+\frac{\varepsilon_{g}^{(J)}}{g_{0}}+i \frac{2 \varepsilon_{\chi}^{(J)}}{\Delta_{-}}\right] .
\end{gathered}
$$

Como o regime dispersivo é aqui tratado, será importante generalizar as expressões através do símbolo de dissintonia $\mathcal{D}=\Delta_{-} /\left|\Delta_{-}\right|= \pm$. O regime AJC consiste no processo de criação de um fóton ao passo que há a mudança do estado atômico fundamental para o estado excitado. Observa-se através do Hamiltoniano efetivo que os estados acoplam-se da forma $\left|\varphi_{0, \mathcal{D}}\right\rangle \leftrightarrow\left|\varphi_{2,-\mathcal{D}}\right\rangle$, o que corresponde à transição entre estados $|g, 0\rangle \rightarrow|e, 1\rangle$ se considerarmos a expansão em ordem zero para $\frac{g_{0}}{\Delta_{-}} \sqrt{n}$. Diante das considerações iniciais, a dinâmica unitária será dada por

$$
\frac{d \tilde{\rho}}{d t}=-i\left[H_{A J C}, \tilde{\rho}\right]
$$

$\operatorname{com}\left|\varphi_{0,-}\right\rangle \equiv\left|\varphi_{0, \mathcal{D}}\right\rangle$

$$
\begin{aligned}
\frac{d}{d t} \tilde{\rho}_{N, M}^{\mathcal{S}, \mathcal{T}}= & -i\left\langle\varphi_{N, \mathcal{S}}\right|\left[\left(\sum_{\mathcal{S}, \mathcal{T}} \sum_{N, M=0}^{\infty} \tilde{\rho}_{N, M}^{\mathcal{S}, \mathcal{T}} H_{e f f}\left|\varphi_{N, \mathcal{S}}\right\rangle\left\langle\varphi_{M, \mathcal{T}}\right|\right)\right. \\
& \left.-\left(\sum_{\mathcal{S}, \mathcal{T}} \sum_{N, M=0}^{\infty} \tilde{\rho}_{N, M}^{\mathcal{S}, \mathcal{T}}\left|\varphi_{N, \mathcal{S}}\right\rangle\left\langle\varphi_{M, \mathcal{T}}\right| H_{e f f}\right)\right]\left|\varphi_{M, \mathcal{T}}\right\rangle \\
= & -i\left[\left(\delta_{N, 0} \delta_{\mathcal{S},-} \theta \tilde{\rho}_{2, M}^{-\mathcal{D}, \mathcal{T}}+\delta_{N, 2} \delta_{\mathcal{S},-\mathcal{D}} \theta^{*} \tilde{\rho}_{0, M}^{-, \mathcal{T}}\right)-\left(\delta_{2, M} \delta_{-\mathcal{D}, \mathcal{T}} \theta \tilde{\rho}_{N, 0}^{\mathcal{S},-}+\delta_{0, M} \delta_{-, \mathcal{T}} \theta^{*} \tilde{\rho}_{N, 2}^{\mathcal{S},-\mathcal{D}}\right)\right] .
\end{aligned}
$$

Escrevendo as equações correspondentes

$$
\begin{gathered}
\frac{d}{d t} \tilde{\rho}_{0,0}^{-,-}=2\left(\theta \tilde{\rho}_{2,0}^{-\mathcal{D},-}\right)^{i}, \\
\frac{d}{d t}\left(\theta \tilde{\rho}_{N=2, M=0}^{-\mathcal{D},-}\right)^{i}=-|\theta|^{2}\left[\tilde{\rho}_{0,0}^{-,-}-\tilde{\rho}_{2,2}^{-\mathcal{D},-\mathcal{D}}\right],
\end{gathered}
$$




$$
\frac{d}{d t} \tilde{\rho}_{2,2}^{-\mathcal{D},-\mathcal{D}}=-2\left(\theta \tilde{\rho}_{2,0}^{-\mathcal{D},-}\right)^{i}
$$

os efeitos da dissipação serão analisados analíticamente.

Inicialmente, a relaxação atômica pura (RAP) será analisada e, para isso, será muito mais simples escrever expressões gerais com o auxílio das equações (4.20), (4.21) e (4.22), além, é claro, do RWA. Portanto as expressões gerais são

$$
\begin{gathered}
\frac{d}{d t} \tilde{\rho}_{0,0}^{-,-}=\gamma\left(\frac{g_{0}^{2}}{\Delta_{-}^{2}} \tilde{\rho}_{1,1}^{\mathcal{D}, \mathcal{D}}+\left(1-\frac{g_{0}^{2}}{\Delta_{-}^{2}}\right) \tilde{\rho}_{1,1}^{-\mathcal{D},-\mathcal{D}}\right) \\
\frac{d}{d t} \tilde{\rho}_{N>0, N}^{\mathcal{D}, \mathcal{D}}=\gamma\left(\left[\frac{g_{0}^{2}}{\Delta_{-}^{2}}(N+1) \tilde{\rho}_{N+1, N+1}^{\mathcal{D}, \mathcal{D}}+\left(1-\frac{g_{0}^{2}}{\Delta_{-}^{2}}(2 N+1)\right) \tilde{\rho}_{N+1, N+1}^{-\mathcal{D}, \mathcal{D}}\right]-\frac{g_{0}^{2}}{\Delta_{-}^{2}} N \tilde{\rho}_{N, N}^{\mathcal{D}, \mathcal{D}}\right), \\
\frac{d}{d t} \tilde{\rho}_{N>0, N}^{-\mathcal{D},-\mathcal{D}}=\gamma\left(\frac{g_{0}^{2}}{\Delta_{-}^{2}} N \tilde{\rho}_{N+1, N+1}^{-\mathcal{D},-\mathcal{D}}-\left(1-\frac{g_{0}^{2}}{\Delta_{-}^{2}} N\right) \tilde{\rho}_{N, N}^{-\mathcal{D},-\mathcal{D}}\right) \\
\frac{d}{d t}\left(\theta \tilde{\rho}_{N>0,0}^{-\mathcal{D},-}\right)^{i}=-\frac{\gamma}{2}\left(1-\frac{g_{0}^{2}}{\Delta_{-}^{2}} N\right)\left(\theta \tilde{\rho}_{N, 0}^{-\mathcal{D},-}\right)^{i}
\end{gathered}
$$

onde foi considerada a expansão em $\frac{g_{0}}{\Delta_{-}}$até segunda ordem! Logo, começando pela equação diferencial para $\tilde{\rho}_{0,0}^{-,-}$e, em seguida, escrevendo as equações para termos que se acoplam, obtem-se

$$
\begin{gathered}
\frac{d}{d t} \tilde{\rho}_{0,0}^{-,-}=2\left(\theta \tilde{\rho}_{2,0}^{-\mathcal{D},-}\right)^{i}+\gamma\left(\frac{g_{0}^{2}}{\Delta_{-}^{2}} \tilde{\rho}_{1,1}^{\mathcal{D} . \mathcal{D}}+\left(1-\frac{g_{0}^{2}}{\Delta_{-}^{2}}\right) \tilde{\rho}_{1,1}^{-\mathcal{D},-\mathcal{D}}\right) \\
\frac{d}{d t}\left(\theta \tilde{\rho}_{2,0}^{-\mathcal{D},-}\right)^{i}=-|\theta|^{2}\left[\tilde{\rho}_{0,0}^{-,-}-\tilde{\rho}_{2,2}^{-\mathcal{D},-\mathcal{D}}\right]-\frac{\gamma}{2}\left(1-\frac{g_{0}^{2}}{\Delta_{-}^{2}} 2\right)\left(\theta \tilde{\rho}_{2,0}^{-\mathcal{D},-}\right)^{i}, \\
\frac{d}{d t} \tilde{\rho}_{1,1}^{\mathcal{D}, \mathcal{D}}=\gamma\left(\left[\frac{g_{0}^{2}}{\Delta_{-}^{2}} 2 \tilde{\rho}_{2,2}^{\mathcal{D}, \mathcal{D}}+\left(1-\frac{g_{0}^{2}}{\Delta_{-}^{2}} 3\right) \tilde{\rho}_{2,2}^{-\mathcal{D},-\mathcal{D}}\right]-\frac{g_{0}^{2}}{\Delta_{-}^{2}} \tilde{\rho}_{1,1}^{\mathcal{D}, \mathcal{D}}\right), \\
\frac{d}{d t} \tilde{\rho}_{1,1}^{-\mathcal{D},-\mathcal{D}}=\gamma\left(\frac{g_{0}^{2}}{\Delta_{-}^{2}} \tilde{\rho}_{2,2}^{-\mathcal{D},-\mathcal{D}}-\left(1-\frac{g_{0}^{2}}{\Delta_{-}^{2}}\right) \tilde{\rho}_{1,1}^{-\mathcal{D},-\mathcal{D}}\right) \\
\frac{d}{d t} \tilde{\rho}_{2,2}^{-\mathcal{D},-\mathcal{D}}=-2\left(\tilde{\rho}_{2,0}^{-\mathcal{D},-} \theta\right)^{i}-\gamma\left(1-\frac{g_{0}^{2}}{\Delta_{-}^{2}} 2\right) \tilde{\rho}_{2,2}^{-\mathcal{D},-\mathcal{D}}, \\
\frac{d}{d t} \tilde{\rho}_{2,2}^{\mathcal{D}, \mathcal{D}}=-\gamma \frac{g_{0}^{2}}{\Delta_{-}^{2}} 2 \tilde{\rho}_{2,2}^{\mathcal{D}, \mathcal{D}} .
\end{gathered}
$$

Fazendo o lado esquerdo das equações igual a zero, assintóticamente obtém-se (com $\left.\frac{g_{0}^{2}}{\Delta_{0}^{2}} \ll 1\right)$

$$
\begin{gathered}
\tilde{\rho}_{2,2}^{\mathcal{D}, \mathcal{D}}=0, \\
\tilde{\rho}_{2,2}^{-\mathcal{D},-\mathcal{D}}=\frac{g_{0}^{2}}{\Delta_{-}^{2}} \tilde{\rho}_{1,1}^{\mathcal{D}, \mathcal{D}},
\end{gathered}
$$




$$
\begin{gathered}
\tilde{\rho}_{1,1}^{-\mathcal{D},-\mathcal{D}}=\frac{g_{0}^{2}}{\Delta_{-}^{2}} \tilde{\rho}_{2,2}^{-\mathcal{D},-\mathcal{D}}=\frac{g_{0}^{4}}{\Delta_{-}^{4}} \tilde{\rho}_{1,1}^{\mathcal{D}, \mathcal{D}}, \\
\left(\theta \tilde{\rho}_{2,0}^{-\mathcal{D},-}\right)^{i}=\frac{-\gamma}{2} \frac{g_{0}^{2}}{\Delta_{-}^{2}} \tilde{\rho}_{1,1}^{\mathcal{D}, \mathcal{D}}, \\
\tilde{\rho}_{0,0}^{-,-}=\frac{g_{0}^{2}}{\Delta_{-}^{2}}\left[1+\left(\frac{\gamma}{2|\theta|}\right)^{2}\right] \tilde{\rho}_{1,1}^{\mathcal{D} \cdot \mathcal{D}} .
\end{gathered}
$$

Usando esse resultado junto à condição de normalização

$$
\tilde{\rho}_{0,0}^{-,-}+\tilde{\rho}_{1,1}^{\mathcal{D}, \mathcal{D}}+\tilde{\rho}_{1,1}^{-\mathcal{D},-\mathcal{D}}+\tilde{\rho}_{2,2}^{-\mathcal{D},-\mathcal{D}}+\tilde{\rho}_{2,2}^{\mathcal{D}, \mathcal{D}}=1
$$

chega-se ao resultado

$$
\tilde{\rho}_{1,1}^{\mathcal{D}, \mathcal{D}}=\left[1+\frac{g_{0}^{2}}{\Delta_{-}^{2}}\left(2+\left(\frac{\gamma}{2|\theta|}\right)^{2}\left(1-2 \frac{g_{0}^{2}}{\Delta_{-}^{2}}\right)\right)\right]^{-1} .
$$

Quando $\gamma \ll|\theta|$ a população tem solução aproximada $\tilde{\rho}_{1,1}^{\mathcal{D}, \mathcal{D}} \approx 1$ e somente um fóton é gerado.

Já para a DAP, generalizando as equações (4.23), (4.24) e (4.25) de acordo com a dissintonia (novamente a generalização das equações será dada em função do símbolo de dissintonia), tem-se

$$
\begin{gathered}
\frac{d}{d t} \tilde{\rho}_{0,0}^{-,-}=0, \\
\frac{d}{d t} \tilde{\rho}_{N>0, N}^{\mathcal{D}, \mathcal{D}}=2 N \gamma_{\phi} \frac{g_{0}^{2}}{\Delta_{-}^{2}}\left[-\tilde{\rho}_{N, N}^{\mathcal{D}, \mathcal{D}}+\tilde{\rho}_{N, N}^{-\mathcal{D},-\mathcal{D}}\right], \\
\frac{d}{d t} \tilde{\rho}_{N>0, N}^{-\mathcal{D},-\mathcal{D}}=2 N \gamma_{\phi} \frac{g_{0}^{2}}{\Delta_{-}^{2}}\left[-\tilde{\rho}_{N, N}^{-\mathcal{D},-\mathcal{D}}+\tilde{\rho}_{N, N}^{\mathcal{D}, \mathcal{D}}\right], \\
\frac{d}{d t}\left(\theta \tilde{\rho}_{N>0,0}^{-\mathcal{D},-}\right)^{i}=-\gamma_{\phi}\left(1-\frac{g_{0}^{2}}{\Delta_{-}^{2}} N\right)\left(\theta \tilde{\rho}_{N, 0}^{-\mathcal{D},-}\right)^{i} .
\end{gathered}
$$

Termos que oscilam rapidamente, $\beta_{n} \gg \gamma_{\phi}$, foram eliminados via RWA. Então, juntos à DU, as equações serão

$$
\begin{gathered}
\frac{d}{d t} \tilde{\rho}_{0,-}^{-,-}=2\left(\theta \tilde{\rho}_{2,0}^{-\mathcal{D},-}\right)^{i} \\
\frac{d}{d t}\left(\theta \tilde{\rho}_{2,0}^{-\mathcal{D},-}\right)^{i}=-|\theta|^{2}\left[\tilde{\rho}_{0,0}^{-,-}-\tilde{\rho}_{2,2}^{-\mathcal{D},-\mathcal{D}}\right]-\gamma_{\phi}\left(1-\frac{g_{0}^{2}}{\Delta_{-}^{2}} 2\right)\left(\theta \tilde{\rho}_{2,0}^{-\mathcal{D},-}\right)^{i} \\
\frac{d}{d t} \tilde{\rho}_{2,2}^{-\mathcal{D},-\mathcal{D}}=-2\left(\theta \tilde{\rho}_{2,0}^{-\mathcal{D},-}\right)^{i}+4 \gamma_{\phi} \frac{g_{0}^{2}}{\Delta_{-}^{2}}\left[-\tilde{\rho}_{2,2}^{-\mathcal{D},-\mathcal{D}}+\tilde{\rho}_{2,2}^{\mathcal{D}, \mathcal{D}}\right] \\
\frac{d}{d t} \tilde{\rho}_{2,2}^{\mathcal{D}, \mathcal{D}}=4 \gamma_{\phi} \frac{g_{0}^{2}}{\Delta_{-}^{2}}\left[-\tilde{\rho}_{2,2}^{\mathcal{D}, \mathcal{D}}+\tilde{\rho}_{2,2}^{-\mathcal{D},-\mathcal{D}}\right]
\end{gathered}
$$


que, quando igualadas a zero, fornecem a solução assintótica

$$
\begin{aligned}
& \left(\theta \tilde{\rho}_{2,0}^{-\mathcal{D},-}\right)^{i}=0, \\
& \tilde{\rho}_{2,2}^{\mathcal{D}, \mathcal{D}}=\tilde{\rho}_{2,2}^{-\mathcal{D},-\mathcal{D}}, \\
& \tilde{\rho}_{0,0}^{-,-}=\tilde{\rho}_{2,2}^{-\mathcal{D},-\mathcal{D}} .
\end{aligned}
$$

Nesse caso, uma nova probabilidade para o sistema transitar surge e altera a evolução do sistema.

$$
\tilde{\rho}_{0,0}^{-,-}=\tilde{\rho}_{2,2}^{-\mathcal{D},-\mathcal{D}}=\tilde{\rho}_{2,2}^{\mathcal{D}, \mathcal{D}}=\frac{1}{3}
$$

Esta seção se encerra com a análise do comportamento assintótico quando há a presença da RPC descrita através EMP. Utilizando as equações para a relaxação da cavidade, além de eliminar termos que oscilam rapidamente e considerar no máximo duas excitações, a formulação geral até segunda ordem é

$$
\begin{gathered}
\frac{d}{d t} \tilde{\rho}_{0,0}^{-,-}=\kappa\left[\left(1-\frac{g_{0}^{2}}{\Delta_{-}^{2}}\right) \tilde{\rho}_{1,1}^{\mathcal{D}, \mathcal{D}}+\frac{g_{0}^{2}}{\Delta_{-}^{2}} \tilde{\rho}_{1,1}^{-\mathcal{D},-\mathcal{D}}\right] \\
\frac{d}{d t} \tilde{\rho}_{N>0, N}^{\mathcal{D}, \mathcal{D}}=\kappa\left((N+1)\left(1-\frac{g_{0}^{2}}{\Delta_{-}^{2}}\right) \tilde{\rho}_{N+1, N+1}^{\mathcal{D}, \mathcal{D}}+\frac{g_{0}^{2}}{\Delta_{-}^{2}} \tilde{\rho}_{N+1, N+1}^{-\mathcal{D},-\mathcal{D}}-N\left(1-\frac{g_{0}^{2}}{\Delta_{-}^{2}}\right) \tilde{\rho}_{N, N}^{\mathcal{D}, \mathcal{D}}\right), \\
\frac{d}{d t} \tilde{\rho}_{N>0, N}^{-\mathcal{D},-\mathcal{D}}=\kappa\left(N\left(1+\frac{g_{0}^{2}}{\Delta_{-}^{2}}\right) \tilde{\rho}_{N+1, N+1}^{-\mathcal{D},-\mathcal{D}}-\left(N-1+\frac{g_{0}^{2}}{\Delta_{-}^{2}} N\right) \tilde{\rho}_{N, N}^{-\mathcal{D},-\mathcal{D}}\right), \\
\frac{d}{d t}\left(\theta \tilde{\rho}_{N>0,0}^{-\mathcal{D},-}\right)^{i}=-\frac{\kappa}{2}\left[N-1+\frac{g_{0}^{2}}{\Delta_{-}^{2}} N\right]\left(\theta \tilde{\rho}_{N, 0}^{-\mathcal{D},-}\right)^{i}
\end{gathered}
$$

Logo, a dinâmica sob a presença deste canal dissipativo fornece

$$
\begin{gathered}
\frac{d}{d t} \tilde{\rho}_{0,0}^{-,-}=2\left(\theta \tilde{\rho}_{2,0}^{-\mathcal{D},-}\right)^{i}+\kappa\left[\left(1-\frac{g_{0}^{2}}{\Delta_{-}^{2}}\right) \tilde{\rho}_{1,1}^{\mathcal{D}, \mathcal{D}}+\frac{g_{0}^{2}}{\Delta_{-}^{2}} \tilde{\rho}_{1,1}^{-\mathcal{D},-\mathcal{D}}\right] \\
\frac{d}{d t}\left(\theta \tilde{\rho}_{2,0}^{-\mathcal{D},-}\right)^{i}=-|\theta|^{2}\left[\tilde{\rho}_{0,0}^{-,-}-\tilde{\rho}_{2,2}^{-\mathcal{D},-\mathcal{D}}\right]-\frac{\kappa}{2}\left[1+2 \frac{g_{0}^{2}}{\Delta_{-}^{2}}\right]\left(\theta \tilde{\rho}_{2,0}^{-\mathcal{D},-}\right)^{i}, \\
\frac{d}{d t} \tilde{\rho}_{1,1}^{\mathcal{D}, \mathcal{D}}=\kappa\left(2\left(1-\frac{g_{0}^{2}}{\Delta_{-}^{2}}\right) \tilde{\rho}_{2,2}^{\mathcal{D}, \mathcal{D}}+\frac{g_{0}^{2}}{\Delta_{-}^{2}} \tilde{\rho}_{2,2}^{-\mathcal{D},-\mathcal{D}}-\left(1-\frac{g_{0}^{2}}{\Delta_{-}^{2}}\right) \tilde{\rho}_{1,1}^{\mathcal{D} \mathcal{D}}\right), \\
\frac{d}{d t} \tilde{\rho}_{1,1}^{-\mathcal{D},-\mathcal{D}}=\kappa\left(\left(1+\frac{g_{0}^{2}}{\Delta_{-}^{2}}\right) \tilde{\rho}_{2,2}^{-\mathcal{D},-\mathcal{D}}-\frac{g_{0}^{2}}{\Delta_{-}^{2}} \tilde{\rho}_{1,1}^{-\mathcal{D},-\mathcal{D}}\right) \\
\frac{d}{d t} \tilde{\rho}_{2,2}^{\mathcal{D}, \mathcal{D}}=-2 \kappa\left(1-\frac{g_{0}^{2}}{\Delta_{-}^{2}}\right) \tilde{\rho}_{2,2}^{\mathcal{D}, \mathcal{D}} \\
\frac{d}{d t} \tilde{\rho}_{2,2}^{-\mathcal{D},-\mathcal{D}}=-2\left(\tilde{\rho}_{2,0}^{-\mathcal{D},-} \theta\right)^{i}-\kappa\left(1+\frac{g_{0}^{2}}{\Delta_{-}^{2}} 2\right) \tilde{\rho}_{2,2}^{-\mathcal{D},-\mathcal{D}}
\end{gathered}
$$


e a solução assintótica poderá ser analisada. Igualando o lado esquerdo das equações acima a zero, o resultado será

$$
\begin{gathered}
\tilde{\rho}_{2,2}^{\mathcal{D}, \mathcal{D}}=0, \\
\tilde{\rho}_{2,2}^{-\mathcal{D},-\mathcal{D}}=\frac{g_{0}^{2}}{\Delta_{-}^{2}} \tilde{\rho}_{1,1}^{-\mathcal{D},-\mathcal{D}}, \\
\tilde{\rho}_{1,1}^{\mathcal{D}, \mathcal{D}}=\frac{g_{0}^{2}}{\Delta_{-}^{2}} \tilde{\rho}_{2,2}^{-\mathcal{D},-\mathcal{D}}=\frac{g_{0}^{4}}{\Delta_{-}^{4}} \tilde{\rho}_{1,1}^{-\mathcal{D},-\mathcal{D}}, \\
\left(\theta \tilde{\rho}_{2,0}^{-\mathcal{D},-}\right)^{i}=\frac{-\kappa}{2} \frac{g_{0}^{2}}{\Delta_{-}^{2}} \tilde{\rho}_{1,1}^{-\mathcal{D},-\mathcal{D}}, \\
\tilde{\rho}_{0,0}^{-,-}=\frac{g_{0}^{2}}{\Delta_{-}^{2}}\left[1+\left(\frac{\kappa}{2|\theta|}\right)^{2}\right] \tilde{\rho}_{1,1}^{-\mathcal{D},-\mathcal{D}} .
\end{gathered}
$$

Nesse caso, a condição de normalização permite concluir que

$$
\tilde{\rho}_{1,1}^{-\mathcal{D},-\mathcal{D}}=\left\{1+\frac{g_{0}^{2}}{\Delta_{-}^{2}}\left(2+\left(\frac{\kappa}{2|\theta|}\right)^{2}\right)\right\}^{-1}
$$

e a condição $\kappa \ll|\theta|$ mostra que uma excitação atômica será gerada com o sistema tendendo ao estado $|e, 0\rangle$, tendo em vista que no regime dispersivo $\left|\Delta_{-}\right| \gg g_{0}$. Entretando, não há completa transferência, dado que $\tilde{\rho}_{0,0}^{-,-} \approx \frac{g_{0}^{2}}{\Delta_{-}^{2}} \tilde{\rho}_{1,1}^{-\mathcal{D},-\mathcal{D}}$.

Quando as relaxações são analisadas em conjunto,

$$
\begin{gathered}
\frac{d}{d t} \tilde{\rho}_{0,0}^{-,-}=2\left(\theta \tilde{\rho}_{2,0}^{-\mathcal{D},-}\right)^{i}+\kappa\left[\left(1-\frac{g_{0}^{2}}{\Delta_{-}^{2}}\right) \tilde{\rho}_{1,1}^{\mathcal{D}, \mathcal{D}}+\frac{g_{0}^{2}}{\Delta_{-}^{2}} \tilde{\rho}_{1,1}^{-\mathcal{D},-\mathcal{D}}\right]+\gamma\left(\frac{g_{0}^{2}}{\Delta_{-}^{2}} \tilde{\rho}_{1,1}^{\mathcal{D}, \mathcal{D}}+\left(1-\frac{g_{0}^{2}}{\Delta_{-}^{2}}\right) \tilde{\rho}_{1,1}^{-\mathcal{D},-\mathcal{D}}\right) \\
\frac{d}{d t}\left(\theta \tilde{\rho}_{2,0}^{-\mathcal{D},-}\right)^{i}=-|\theta|^{2}\left[\tilde{\rho}_{0,0}^{-,-}-\tilde{\rho}_{2,2}^{-\mathcal{D},-\mathcal{D}}\right]-\frac{\kappa}{2}\left[1+2 \frac{g_{0}^{2}}{\Delta_{-}^{2}}\right]\left(\theta \tilde{\rho}_{2,0}^{-\mathcal{D},-}\right)^{i}-\frac{\gamma}{2}\left(1-\frac{g_{0}^{2}}{\Delta_{-}^{2}} 2\right)\left(\theta \tilde{\rho}_{2,0}^{-\mathcal{D},-}\right)^{i} \\
\frac{d}{d t} \tilde{\rho}_{2,2}^{-\mathcal{D},-\mathcal{D}}=-2(\theta .61) \\
\frac{d}{d t} \tilde{\rho}_{2,2}^{\mathcal{D}, \mathcal{D}}=-2 \kappa\left(1-\frac{g_{0}^{2}}{\Delta_{-}^{2}}\right) \tilde{\rho}_{2,-}^{\mathcal{D}, \mathcal{D}}-2 \gamma \frac{g_{0}^{2}}{\Delta_{-}^{2}} \tilde{\rho}_{2,2}^{\mathcal{D}, \mathcal{D}} \\
\frac{d}{d t} \tilde{\rho}_{1,1}^{\mathcal{D}, \mathcal{D}}=\kappa\left(1+\frac{g_{0}^{2}}{\Delta_{-}^{2}} 2\right) \tilde{\rho}_{2,2}^{-\mathcal{D},-\mathcal{D}}-\gamma\left(2\left(1-\frac{g_{0}^{2}}{\Delta_{-}^{2}}\right) \tilde{\rho}_{2,2}^{-\mathcal{D}, \mathcal{D}}+\frac{g_{0}^{2}}{\Delta_{-}^{2}} \tilde{\rho}_{2,2}^{-\mathcal{D},-\mathcal{D}}-\left(1-\frac{g_{0}^{2}}{\Delta_{-}^{2}}\right) \tilde{\rho}_{1,1}^{\mathcal{D}, \mathcal{D}}\right) \\
+\gamma\left(\left[\frac{g_{0}^{2}}{\Delta_{-}^{2}} 2 \tilde{\rho}_{2,2}^{\mathcal{D}, \mathcal{D}}+\left(1-\frac{g_{0}^{2}}{\Delta_{-}^{2}} 3\right) \tilde{\rho}_{2,2}^{-\mathcal{D},-\mathcal{D}}\right]-\frac{g_{0}^{2}}{\Delta_{-}^{2}} \tilde{\rho}_{1,1}^{\mathcal{D}, \mathcal{D}}\right) \\
\frac{d}{d t} \tilde{\rho}_{1,1}^{-\mathcal{D},-\mathcal{D}}=\kappa\left(\left(1+\frac{g_{0}^{2}}{\Delta_{-}^{2}}\right) \tilde{\rho}_{2,2}^{-\mathcal{D},-\mathcal{D}}-\frac{g_{0}^{2}}{\Delta_{-}^{2}} \tilde{\rho}_{1,1}^{-\mathcal{D},-\mathcal{D}}\right)+\gamma\left(\frac{g_{0}^{2}}{\Delta_{-}^{2}} \tilde{\rho}_{2,2}^{-\mathcal{D},-\mathcal{D}}-\left(1-\frac{g_{0}^{2}}{\Delta_{-}^{2}}\right) \tilde{\rho}_{1,1}^{-\mathcal{D},-\mathcal{D}}\right)
\end{gathered}
$$


obtem-se como solução assintótica

$$
\begin{aligned}
& \tilde{\rho}_{2,2}^{\mathcal{D}, \mathcal{D}}=0, \\
& \tilde{\rho}_{2,2}^{-\mathcal{D},-\mathcal{D}}=\frac{\kappa\left(1-\frac{g_{0}^{2}}{\Delta_{-}^{2}}\right)+\gamma \frac{g_{0}^{2}}{\Delta_{-}^{2}}}{\kappa \frac{g_{0}^{2}}{\Delta_{-}^{2}}+\gamma\left(1-\frac{g_{0}^{2}}{\Delta_{-}^{2}} 3\right)} \tilde{\rho}_{1,1}^{\mathcal{D}, \mathcal{D}}, \\
& \tilde{\rho}_{1,1}^{-\mathcal{D},-\mathcal{D}}=\frac{\left[\kappa\left(1+\frac{g_{0}^{2}}{\Delta_{-}^{2}}\right)+\gamma \frac{g_{0}^{2}}{\Delta_{-}^{2}}\right]\left[\kappa\left(1-\frac{g_{0}^{2}}{\Delta_{-}^{2}}\right)+\gamma \frac{g_{0}^{2}}{\Delta_{-}^{2}}\right]}{\left[\kappa \frac{g_{0}^{2}}{\Delta_{-}^{2}}+\gamma\left(1-\frac{g_{0}^{2}}{\Delta_{-}^{2}}\right)\right]} \tilde{\rho}_{1,1}^{\mathcal{D}, \mathcal{D}} \\
& =\frac{\kappa\left(1+\frac{g_{0}^{2}}{\Delta_{-}^{2}}\right)+\gamma \frac{g_{0}^{2}}{\Delta_{-}^{2}}}{\kappa \frac{g_{0}^{2}}{\Delta_{-}^{2}}+\gamma\left(1-\frac{g_{0}^{2}}{\Delta_{-}^{2}}\right)} \tilde{\rho}_{2,2}^{-\mathcal{D},-\mathcal{D}}, \\
& \left(\theta \tilde{\rho}_{2,0}^{-\mathcal{D},-}\right)^{i}=\frac{-1}{2}\left[\kappa\left(1+2 \frac{g_{0}^{2}}{\Delta_{-}^{2}}\right)+\gamma\left(1-2 \frac{g_{0}^{2}}{\Delta_{-}^{2}}\right)\right] \frac{\left[\kappa\left(1-\frac{g_{0}^{2}}{\Delta_{-}^{2}}\right)+\gamma \frac{g_{0}^{2}}{\Delta_{-}^{2}}\right]}{\left[\kappa \frac{g_{0}^{2}}{\Delta_{-}^{2}}+\gamma\left(1-\frac{g_{0}^{2}}{\Delta_{-}^{2}} 3\right)\right]} \tilde{\rho}_{1,1}^{\mathcal{D}, \mathcal{D}}, \\
& \tilde{\rho}_{0,0}^{-,-}=\left(1+\left[\frac{\kappa\left(1+2 \frac{g_{0}^{2}}{\Delta_{0}^{2}}\right)+\gamma\left(1-2 \frac{g_{0}^{2}}{\Delta_{0}^{2}}\right)}{2|\theta|}\right]^{2}\right) \frac{\left[\kappa\left(1-\frac{g_{0}^{2}}{\Delta_{-}^{2}}\right)+\gamma \frac{g_{0}^{2}}{\Delta_{-}^{2}}\right]}{\left[\kappa \frac{g_{0}^{2}}{\Delta_{-}^{2}}+\gamma\left(1-3 \frac{g_{0}^{2}}{\Delta_{-}^{2}}\right)\right]} \tilde{\rho}_{1,1}^{\mathcal{D}, \mathcal{D}}, \\
& =\left\{1+\left[\frac{\kappa+\gamma+2 \frac{g_{0}^{2}}{\Delta_{-}^{2}}(\kappa-\gamma)}{2|\theta|}\right]^{2}\right\} \tilde{\rho}_{2,2}^{-\mathcal{D},-\mathcal{D}} \text {. }
\end{aligned}
$$

Probabilidades não-nulas são atingidas, de forma que a DU é novamente modificada. Podemos checar facilmente que para $\gamma=0$ ou $\kappa=0$ teremos as soluções obtidas previamente. Ademais, caso a solução para ambas as relaxações seja tomada até primeira ordem em $\frac{g_{0}}{\Delta_{-}}$e $\gamma \sim \kappa$ a DU é afetada de tal forma que o sistema tem estados com probabilidades $(\gamma, \kappa \ll|\theta|)$

$$
\begin{gathered}
\tilde{\rho}_{2,2}^{-\mathcal{D},-\mathcal{D}}=\left[2+\frac{\gamma}{\kappa}+\frac{\kappa}{\gamma}\right]^{-1} \sim \frac{1}{4} \\
\tilde{\rho}_{0,0}^{-,-}=\left[2+\frac{\gamma}{\kappa}+\frac{\kappa}{\gamma}\right]^{-1} \sim \frac{1}{4} \\
\tilde{\rho}_{1,1}^{\mathcal{D}, \mathcal{D}}=\frac{P_{a}}{P_{c}}\left[2+\frac{\gamma}{\kappa}+\frac{\kappa}{\gamma}\right]^{-1} \sim \frac{1}{4} \\
\tilde{\rho}_{1,1}^{-\mathcal{D},-\mathcal{D}}=\frac{P_{c}}{P_{a}}\left[2+\frac{\gamma}{\kappa}+\frac{\kappa}{\gamma}\right]^{-1} \sim \frac{1}{4} .
\end{gathered}
$$




\subsubsection{Regime anti-efeito Casimir dinâmico (AECD)}

O regime anti-ECD previsto recentemente por $[43,49]$ ocorre no $\mathrm{RD}$ e consiste na aniquilação coerente de duas excitações do sistema via acoplamento $\left|\varphi_{k, \mathcal{D}}\right\rangle \leftrightarrow\left|\varphi_{k-2,-\mathcal{D}}\right\rangle$ para um dado valor $k \geq 3$ ou, a grosso modo, uma transição equivalente a $|g, k\rangle \leftrightarrow|e, k-3\rangle$, levando em conta a aproximação em ordem zero em $\frac{g_{0}}{\Delta_{-}} \sqrt{n}$ no RD. Em palavras, o que foi afirmado na setença anterior pode ser entendido como três fótons sendo aniquilados ao passo que uma excitação atômica é criada. Como visto, esse efeito é implementado com a modulação da frequência $\eta_{k}^{(A)}=3 \omega_{0}-\Omega_{0}+2\left(\delta_{-}-\delta_{+}\right)(k-1) \simeq$ $\lambda_{k, \mathcal{D}}-\lambda_{k-2,-\mathcal{D}}$ e fornece através de (2.17) o Hamiltoniano efetivo

$$
\tilde{H}=\left(\theta\left|\varphi_{k-2,-\mathcal{D}}\right\rangle\left\langle\varphi_{k, \mathcal{D}}\left|+\theta^{*}\right| \varphi_{k, \mathcal{D}}\right\rangle\left\langle\varphi_{k-2,-\mathcal{D}}\right|\right)
$$

com

$$
\begin{aligned}
\theta & =-i \mathcal{D} \frac{\delta_{-} \Omega_{0} g_{0}}{2 \omega_{0} \Delta_{-}} \sqrt{k(k-1)(k-2)} \Upsilon^{(A)} \\
\Upsilon^{(A)} & =\left[\frac{\varepsilon_{\omega}^{(A)}}{2 \omega_{0}+\Delta_{-}}+\frac{\omega_{0}+\Delta_{-}}{2 \omega_{0}+\Delta_{-}} \frac{\varepsilon_{\Omega}^{(A)}}{\Omega_{0}}-\frac{\varepsilon_{g}^{(A)}}{g_{0}}\right] .
\end{aligned}
$$

Nesse regime, a DU é obtida considerando-se inicialmente o sistema em um dado estado com probabilidade $\tilde{\rho}_{k, k}^{\mathcal{D}, \mathcal{D}}$, de forma que sua equação e as demais que a acoplam sejam

$$
\begin{gathered}
\frac{d}{d t} \tilde{\rho}_{k, k}^{\mathcal{D}, \mathcal{D}}=2\left(\theta \tilde{\rho}_{k-2, k}^{-\mathcal{D}, \mathcal{D}}\right)^{i}, \\
\frac{d}{d t}\left(\theta \tilde{\rho}_{k-2, k}^{-\mathcal{D}, \mathcal{D}}\right)^{i}=-|\theta|^{2}\left[\tilde{\rho}_{k, k}^{\mathcal{D}, \mathcal{D}}-\tilde{\rho}_{k-2, k-2}^{-\mathcal{D},-\mathcal{D}}\right], \\
\frac{d}{d t} \tilde{\rho}_{k-2, k-2}^{-\mathcal{D},-\mathcal{D}}=-2\left(\theta \tilde{\rho}_{k-2, k}^{-\mathcal{D}, \mathcal{D}}\right)^{i} .
\end{gathered}
$$

A RAP tem como equações gerais

$$
\begin{gathered}
\frac{d}{d t} \tilde{\rho}_{N>0, N}^{\mathcal{D}, \mathcal{D}}=\gamma\left(\left[\frac{g_{0}^{2}}{\Delta_{-}^{2}}(N+1) \tilde{\rho}_{N+1, N+1}^{\mathcal{D}, \mathcal{D}}+\left(1-\frac{g_{0}^{2}}{\Delta_{-}^{2}}(2 N+1)\right) \tilde{\rho}_{N+1, N+1}^{-\mathcal{D},-\mathcal{D}}\right]-\frac{g_{0}^{2}}{\Delta_{0}^{2}} N \tilde{\rho}_{N, N}^{\mathcal{D}, \mathcal{D}}\right), \\
\frac{d}{d t} \tilde{\rho}_{N>0, N}^{-\mathcal{D},-\mathcal{D}}=\gamma\left(\frac{g_{0}^{2}}{\Delta_{-}^{2}} N \tilde{\rho}_{N+1, N+1}^{-\mathcal{D},-\mathcal{D}}-\left(1-\frac{g_{0}^{2}}{\Delta_{-}^{2}} N\right) \tilde{\rho}_{N, N}^{-\mathcal{D},-\mathcal{D}}\right), \\
\frac{d}{d t}\left(\theta \tilde{\rho}_{N>0, M>0, M \neq N}^{-\mathcal{D}, \mathcal{D}}\right)^{i}=-\frac{\gamma}{2}\left(1+\frac{g_{0}^{2}}{\Delta_{-}^{2}}(M-N)\right)\left(\theta \tilde{\rho}_{N, M}^{-\mathcal{D}, \mathcal{D}}\right)^{i} .
\end{gathered}
$$


Quando a DU está sob RAP e o comportamento é analisado no regime assintótico e em primeira ordem em $g_{0} / \Delta_{-}$, as equações que a descrevem serão

$$
\begin{gathered}
\frac{d}{d t} \tilde{\rho}_{k+1, k+1}^{-\mathcal{D},-\mathcal{D}}=-\gamma \tilde{\rho}_{k+1, k+1}^{-\mathcal{D},-\mathcal{D}}, \\
\frac{d}{d t} \tilde{\rho}_{k, k}^{\mathcal{D}, \mathcal{D}}=2\left(\theta \tilde{\rho}_{k-2, k}^{-\mathcal{D}, \mathcal{D}}\right)^{i}+\gamma \tilde{\rho}_{k+1, k+1}^{-\mathcal{D},-\mathcal{D}}, \\
\frac{d}{d t} \tilde{\rho}_{k, k}^{-\mathcal{D},-\mathcal{D}}=-\gamma \tilde{\rho}_{k, k}^{-\mathcal{D},-\mathcal{D}}, \\
\frac{d}{d t}\left(\theta \tilde{\rho}_{k-2, k}^{-\mathcal{D}, \mathcal{D}}\right)^{i}=-|\theta|^{2}\left[\tilde{\rho}_{k, k}^{\mathcal{D}, \mathcal{D}}-\tilde{\rho}_{k-2, k-2}^{-\mathcal{D},-\mathcal{D}}\right]-\frac{\gamma}{2}\left(\theta \tilde{\rho}_{k-2, k}^{-\mathcal{D}, \mathcal{D}}\right)^{i}, \\
\frac{d}{d t} \tilde{\rho}_{k-1, k-1}^{\mathcal{D}, \mathcal{D}}=\gamma \tilde{\rho}_{k, k}^{-\mathcal{D},-\mathcal{D}}, \\
\frac{d}{d t} \tilde{\rho}_{k-1, k-1}^{-\mathcal{D},-\mathcal{D}}=-\gamma \tilde{\rho}_{k-1, k-1}^{-\mathcal{D},-\mathcal{D}}, \\
\frac{d}{d t} \tilde{\rho}_{k-2, k-2}^{\mathcal{D}, \mathcal{D}}=\gamma \tilde{\rho}_{k-1, k-1}^{-\mathcal{D},-\mathcal{D}}, \\
\frac{d}{d t} \tilde{\rho}_{k-2, k-2}^{-\mathcal{D},-\mathcal{D}}=-2\left(\theta \tilde{\rho}_{k-2, k}^{-\mathcal{D}, \mathcal{D}}\right)^{i}-\gamma \tilde{\rho}_{k-2, k-2}^{-\mathcal{D},-\mathcal{D}}, \\
\frac{d}{d t} \tilde{\rho}_{k-3, k-3}^{\mathcal{D}, \mathcal{D}}=\gamma \tilde{\rho}_{k-2, k-2}^{-\mathcal{D}, \mathcal{D}}, \\
\frac{d}{d t} \tilde{\rho}_{k-3, k-3}^{-\mathcal{D}, \mathcal{D}}=-\gamma \tilde{\rho}_{k-3, k-3}^{-\mathcal{D},-\mathcal{D}} .
\end{gathered}
$$

Observa-se que a única solução não-nula será para a população $\tilde{\rho}_{k-3, k-3}^{\mathcal{D}, \mathcal{D}}$. Para o caso $k=3$, observase que o sistema vai assintóticamente para o EZE, diferentemente do visto anteriormente em outros efeitos em que havia equilíbrio em estados que não fossem o EZE. Se a solução for tomada para a próxima ordem em $\frac{g_{0}}{\Delta_{-}}$e $k>3$ para largas escalas de tempo teremos também $\tilde{\rho}_{k-3, k-3}^{\mathcal{D}, \mathcal{D}}=0$ e a única solução assintótica não-nula será o estado $\tilde{\rho}_{0,0}^{\mathcal{D}} \mathcal{D}$.

Já para a DAP, com equações gerais

$$
\begin{gathered}
\frac{d}{d t} \tilde{\rho}_{N>0, N}^{\mathcal{D}, \mathcal{D}}=2 N \gamma_{\phi} \frac{g_{0}^{2}}{\Delta_{-}^{2}}\left[-\tilde{\rho}_{N, N}^{\mathcal{D}, \mathcal{D}}+\tilde{\rho}_{N, N}^{-\mathcal{D},-\mathcal{D}}\right] \\
\frac{d}{d t} \tilde{\rho}_{N>0, N}^{-\mathcal{D},-\mathcal{D}}=2 N \gamma_{\phi} \frac{g_{0}^{2}}{\Delta_{-}^{2}}\left[-\tilde{\rho}_{N, N}^{-\mathcal{D},-\mathcal{D}}+\tilde{\rho}_{N, N}^{\mathcal{D}, \mathcal{D}}\right] \\
\frac{d}{d t}\left(\theta \tilde{\rho}_{N>0, M>0, M \neq N}^{-\mathcal{D}, \mathcal{D}}\right)^{i}=-\gamma_{\phi}\left(1-\frac{g_{0}^{2}}{\Delta_{-}^{2}}(N+M)\right)\left(\theta \tilde{\rho}_{N, M}^{-\mathcal{D}, \mathcal{D}}\right)^{i},
\end{gathered}
$$

a dinâmica sob dissipação terá como equações

$$
\frac{d}{d t} \tilde{\rho}_{k, k}^{\mathcal{D}, \mathcal{D}}=2\left(\theta \tilde{\rho}_{k-2, k}^{-\mathcal{D}, \mathcal{D}}\right)^{i}+2 k \gamma_{\phi} \frac{g_{0}^{2}}{\Delta_{-}^{2}}\left[-\tilde{\rho}_{k, k}^{\mathcal{D}, \mathcal{D}}+\tilde{\rho}_{k, k}^{-\mathcal{D},-\mathcal{D}}\right],
$$




$$
\begin{gathered}
\frac{d}{d t} \tilde{\rho}_{k, k}^{-\mathcal{D},-\mathcal{D}}=2 k \gamma_{\phi} \frac{g_{0}^{2}}{\Delta_{-}^{2}}\left[-\tilde{\rho}_{k, k}^{-\mathcal{D},-\mathcal{D}}+\tilde{\rho}_{k, k}^{\mathcal{D}, \mathcal{D}}\right], \\
\frac{d}{d t}\left(\theta \tilde{\rho}_{k-2, k}^{-\mathcal{D}, \mathcal{D}}\right)^{i}=-|\theta|^{2}\left[\tilde{\rho}_{k, k}^{\mathcal{D}, \mathcal{D}}-\tilde{\rho}_{k-2, k-2}^{-\mathcal{D},-\mathcal{D}}\right]-\gamma_{\phi}\left(1-\frac{g_{0}^{2}}{\Delta_{-}^{2}} 2(k-1)\right)\left(\theta \tilde{\rho}_{k-2, k}^{-\mathcal{D}, \mathcal{D}}\right)^{i}, \\
\frac{d}{d t} \tilde{\rho}_{k-2, k-2}^{-\mathcal{D},-\mathcal{D}}=-2\left(\theta \tilde{\rho}_{k-2, k}^{-\mathcal{D}, \mathcal{D}}\right)^{i}+2(k-2) \gamma_{\phi} \frac{g_{0}^{2}}{\Delta_{-}^{2}}\left[-\tilde{\rho}_{k-2, k-2}^{-\mathcal{D},-\mathcal{D}}+\tilde{\rho}_{k-2, k-2}^{\mathcal{D}, \mathcal{D}}\right], \\
\frac{d}{d t} \tilde{\rho}_{k-2, k-2}^{\mathcal{D}, \mathcal{D}}=2(k-2) \gamma_{\phi} \frac{g_{0}^{2}}{\Delta_{-}^{2}}\left[-\tilde{\rho}_{k-2, k-2}^{\mathcal{D}, \mathcal{D}}+\tilde{\rho}_{k-2, k-2}^{-\mathcal{D},-\mathcal{D}}\right] .
\end{gathered}
$$

A solução assintótica será

$$
\begin{gathered}
\tilde{\rho}_{k, k}^{\mathcal{D}, \mathcal{D}}=\tilde{\rho}_{k, k}^{-\mathcal{D},-\mathcal{D}}, \\
\left(\theta \tilde{\rho}_{k-2, k}^{-\mathcal{D}, \mathcal{D}}\right)^{i}=0, \\
\tilde{\rho}_{k-2, k-2}^{\mathcal{D}, \mathcal{D}}=\tilde{\rho}_{k-2, k-2}^{-\mathcal{D},-\mathcal{D}}, \\
\tilde{\rho}_{k, k}^{\mathcal{D}, \mathcal{D}}=\tilde{\rho}_{k-2, k-2}^{-\mathcal{D},-\mathcal{D}},
\end{gathered}
$$

enquanto a normalização

$$
\tilde{\rho}_{k, k}^{-\mathcal{D},-\mathcal{D}}=\tilde{\rho}_{k, k}^{\mathcal{D}, \mathcal{D}}=\tilde{\rho}_{k-2, k-2}^{-\mathcal{D},-\mathcal{D}}=\tilde{\rho}_{k-2, k-2}^{\mathcal{D}, \mathcal{D}}
$$

mostra como o comportamento esperado será completamente alterado pela defasagem para longas escalas de tempo.

A RPC tem expressões gerais da forma

$$
\begin{gathered}
\frac{d}{d t} \tilde{\rho}_{N>0, N}^{\mathcal{D}, \mathcal{D}}=\kappa\left((N+1)\left(1-\frac{g_{0}^{2}}{\Delta_{-}^{2}}\right) \tilde{\rho}_{N+1, N+1}^{\mathcal{D}, \mathcal{D}}+\frac{g_{0}^{2}}{\Delta_{-}^{2}} \tilde{\rho}_{N+1, N+1}^{-\mathcal{D},-\mathcal{D}}-N\left(1-\frac{g_{0}^{2}}{\Delta_{-}^{2}}\right) \tilde{\rho}_{N, N}^{\mathcal{D}, \mathcal{D}}\right), \\
\frac{d}{d t} \tilde{\rho}_{N>0, N}^{-\mathcal{D},-\mathcal{D}}=\kappa\left(N\left(1+\frac{g_{0}^{2}}{\Delta_{-}^{2}}\right) \tilde{\rho}_{N+1, N+1}^{-\mathcal{D},-\mathcal{D}}-\left(N-1+\frac{g_{0}^{2}}{\Delta_{-}^{2}} N\right) \tilde{\rho}_{N, N}^{-\mathcal{D},-\mathcal{D}}\right), \\
\frac{d}{d t}\left(\theta \tilde{\rho}_{N>0, M \neq N}^{-\mathcal{D}, \mathcal{D}}\right)^{i}=-\frac{\kappa}{2}\left[N+M-\left(1-\frac{g_{0}^{2}}{\Delta_{-}^{2}} N\right)+\frac{g_{0}}{\Delta_{-}} \sqrt{M}\right]\left(\theta \tilde{\rho}_{N>0, M \neq N}^{-\mathcal{D}, \mathcal{D}}\right)^{i} .
\end{gathered}
$$

Nesse caso, não é interessante a análise da RPC, pois a única solução não-nula será o EZE. Como o AECD é um efeito muito fraco e $|g, 0\rangle$ não acopla com nenhum outro estado, teremos fótons sendo perdidos para o reservatório em um decaimento praticamente exponencial. 


\subsection{Comportamento para quatro excitações}

\subsubsection{Geração de fótons do vácuo incrementada no regime ressonante}

Voltando ao RR, vamos considerar uma modulação multi-frequência ou, mais especificamente, dupla modulação em $\Omega$ e $g$, com as frequências dadas por $\eta^{(r)}=2 \omega_{0}+\mathcal{R} g_{0} \sqrt{2}$ e $\eta^{(r 2)}=2 \omega_{0}+$ $g_{0}\left(2 \mathcal{R}_{2}-\sqrt{2} \mathcal{R}\right)$, com $\mathcal{R}, \mathcal{R}_{2}= \pm$. A modulação $\eta^{(r 2)}$ é ressonante quando $\eta^{(j)}=\lambda_{4, \mathcal{R}_{2}}-\lambda_{2, \mathcal{R}}$ e nesse caso o Hamiltoniano efetivo se torna

$$
\begin{aligned}
\tilde{H} & =\sum_{m, \mathcal{S}, \mathcal{T}} \sum_{\eta^{(r)}, \eta^{(r 2)}} \Xi_{m, \mathcal{T}, \mathcal{S}}^{(j)} e^{-i t\left(\lambda_{m+2, \mathcal{S}}-\lambda_{m, \mathcal{T}}-\eta^{(j)}\right)}\left|\varphi_{m, \mathcal{T}}\right\rangle\left\langle\varphi_{m+2, \mathcal{S}}\right|+h . c . \\
& =\theta\left|\varphi_{0,-}\right\rangle\left\langle\varphi_{2, \mathcal{R}}\left|+\theta^{*}\right| \varphi_{2, \mathcal{R}}\right\rangle\left\langle\varphi_{0,-}\left|+\theta_{2}\right| \varphi_{2, \mathcal{R}}\right\rangle\left\langle\varphi_{4, \mathcal{R}_{2}}\left|+\theta_{2}^{*}\right| \varphi_{4, \mathcal{R}_{2}}\right\rangle\left\langle\varphi_{2, \mathcal{R}}\right|
\end{aligned}
$$

Lembrando que no RR $\Delta_{-}=0, \theta_{0}=0, \theta_{n>0}=\pi / 4$, aqui será considerado no máximo quatro excitações, além, é claro, do método RWA para eliminar termos que oscilam rapidamente. Os termos $\theta$ e $\theta_{2}$ são dados por

$$
\theta=i \mathcal{R} \frac{1}{4} \sqrt{2} g_{0} \Upsilon^{(r)}, \theta_{2}=\Xi_{2, \mathcal{R}, \mathcal{R}_{2}}^{(r 2)}=-i \mathcal{R}_{2} \frac{\sqrt{3}}{4} g_{0} \Upsilon^{(r 2)},
$$

onde para $\theta$ será considerada a modulação para $\Omega$ e para $\theta_{2}$ o parâmetro $g$, sendo

$$
\begin{aligned}
\Upsilon^{(r)} & =\frac{\varepsilon_{\omega}^{(r)}}{2 \omega_{0}}+\frac{\varepsilon_{\Omega}^{(r)}}{2 \omega_{0}}-\frac{\varepsilon_{g}^{(r)}}{g_{0}}+\mathcal{R} i \frac{\sqrt{2} \varepsilon_{\chi}^{(r)}}{g_{0}}, \\
\Upsilon^{(r 2)} & =\frac{\varepsilon_{\omega}^{(r 2)}}{2 \omega_{0}}+\frac{\varepsilon_{\Omega}^{(r 2)}}{2 \omega_{0}}-\frac{\varepsilon_{g}^{(r 2)}}{g_{0}}+\mathcal{R}_{2} i \frac{\sqrt{2} \varepsilon_{\chi}^{(r 2)}\left(\sqrt{2}+\mathcal{R} \mathcal{R}_{2}\right)}{g_{0}} .
\end{aligned}
$$

Nesse modelo, a DU é descrita por

$$
\begin{aligned}
\frac{d}{d t} \tilde{\rho}_{N, M}^{\mathcal{S}, \mathcal{T}}= & -i\left[\left(\delta_{N, 0} \delta_{\mathcal{S},-} \theta \tilde{\rho}_{2, M}^{\mathcal{R}, \mathcal{T}}+\delta_{N, 2} \delta_{\mathcal{S}, \mathcal{R}} \theta^{*} \tilde{\rho}_{0, M}^{-, \mathcal{T}}+\delta_{N, 2} \delta_{\mathcal{S}, \mathcal{R}} \theta_{2} \tilde{\rho}_{4, M}^{\mathcal{R}_{2}, \mathcal{T}}+\delta_{N, 4} \delta_{\mathcal{S}, \mathcal{R}_{2}} \theta^{*} \tilde{\rho}_{2, M}^{\mathcal{R}, \mathcal{T}}\right)\right. \\
& \left.-\left(\delta_{M, 2} \delta_{\mathcal{R}, \mathcal{T}} \theta \tilde{\rho}_{N, 0}^{\mathcal{S}_{N},-}+\delta_{M, 0} \delta_{\mathcal{T},-} \theta^{*} \tilde{\rho}_{N, 2}^{\mathcal{S}, \mathcal{R}}+\delta_{M, 4} \delta_{\mathcal{T}, \mathcal{R}_{2}} \theta_{2} \tilde{\rho}_{N, 2}^{\mathcal{S}, \mathcal{R}}+\delta_{M, 2} \delta_{\mathcal{T}, \mathcal{R}} \theta_{2}^{*} \tilde{\rho}_{N, 4}^{\mathcal{S}, \mathcal{R}_{2}}\right)\right]
\end{aligned}
$$

que torna possível escrever as equações da parte unitária partindo do EZE

$$
\begin{gathered}
\frac{d}{d t} \tilde{\rho}_{0,0}^{-,-}=2\left(\theta \tilde{\rho}_{2,0}^{\mathcal{R},-}\right)^{i} \\
\frac{d}{d t}\left(\theta \tilde{\rho}_{2,0}^{\mathcal{R},-}\right)^{i}=-|\theta|^{2} \tilde{\rho}_{0,0}^{-,-}-\left(\theta \theta_{2} \tilde{\rho}_{4,0}^{\mathcal{R}_{2,-}}\right)^{r}+|\theta|^{2} \tilde{\rho}_{2,2}^{\mathcal{R}, \mathcal{R}} \\
\frac{d}{d t}\left(\theta \theta_{2} \tilde{\rho}_{4,0}^{\mathcal{R}_{2},-}\right)^{r}=\left|\theta_{2}\right|^{2}\left(\theta \tilde{\rho}_{2,0}^{\mathcal{R},-}\right)^{i}-|\theta|^{2}\left(\theta_{2} \tilde{\rho}_{4,2}^{\mathcal{R}_{2}, \mathcal{R}}\right)^{i}
\end{gathered}
$$




$$
\begin{gathered}
\frac{d}{d t}\left(\theta_{2} \tilde{\rho}_{4,2}^{\mathcal{R}_{2}, \mathcal{R}}\right)^{i}=-\left|\theta_{2}\right|^{2} \tilde{\rho}_{2,2}^{\mathcal{R}, \mathcal{R}}+\left(\theta \theta_{2} \tilde{\rho}_{4,0}^{\mathcal{R}_{2},-}\right)^{r}+\left|\theta_{2}\right|^{2} \tilde{\rho}_{4,4}^{\mathcal{R}_{2}, \mathcal{R}_{2}} \\
\frac{d}{d t} \tilde{\rho}_{2,2}^{\mathcal{R}, \mathcal{R}}=2\left(\theta_{2} \tilde{\rho}_{4,2}^{\mathcal{R}_{2}, \mathcal{R}}\right)^{i}-2\left(\theta \tilde{\rho}_{2,0}^{\mathcal{R},-}\right)^{i} \\
\frac{d}{d t} \tilde{\rho}_{4,4}^{\mathcal{R}_{2}, \mathcal{R}_{2}}=-2\left(\theta_{2} \tilde{\rho}_{4,2}^{\mathcal{R}_{2}, \mathcal{R}}\right)^{i}
\end{gathered}
$$

O índice sobrescrito $r$ em $\left(\theta \theta_{2} \tilde{\rho}_{4,0}^{\mathcal{R}_{2},-}\right)^{r}$ indica que a parte real foi tomada. Uma solução analítica para a DU pode ser obtida expandindo-se a função de onda na representação de interação

$$
|\tilde{\Psi}\rangle=A\left|\varphi_{0,-}\right\rangle+B\left|\varphi_{2, \mathcal{R}}\right\rangle+C\left|\varphi_{4, \mathcal{R}_{2}}\right\rangle+\sum
$$

onde $\sum$ engloba todas as outras amplitudes de probabilidade não acopladas pelo Hamiltoniano. A equação de Schrödinger

$$
i \frac{d}{d t}|\tilde{\Psi}\rangle=\tilde{H}|\tilde{\Psi}\rangle=-i\left(\theta B\left|\varphi_{0,-}\right\rangle+\theta^{*} A\left|\varphi_{2, \mathcal{R}}\right\rangle+\theta_{2}^{*} B\left|\varphi_{4, \mathcal{R}_{2}}\right\rangle+\theta_{2} C\left|\varphi_{2, \mathcal{R}}\right\rangle\right)
$$

fornece

$$
\begin{gathered}
\dot{A}=-i \theta B, \\
\dot{B}=-i \theta^{*} A-i \theta_{2} C, \\
\dot{C}=-i \theta_{2}^{*} B .
\end{gathered}
$$

A solução para $B_{0}=0$ é

$$
\begin{aligned}
A= & \left(A_{0}+\frac{\theta_{2}}{\theta^{*}} C_{0}\right) \cos R t-\frac{\theta_{2}}{R^{2} \theta^{*}}\left[2 \theta_{2}^{*}\left(\theta^{*} A_{0}+\theta_{2} C_{0}\right) \cos ^{2} \frac{1}{2} R t\right. \\
& \left.-2 \theta^{*} \theta_{2}^{*} A_{0}+\left(R^{2}-2\left|\theta_{2}\right|^{2}\right) C_{0}\right], \\
B= & -i \frac{\left(\theta^{*} A_{0}+\theta_{2} C_{0}\right) \sin R t}{R}, \\
C= & \frac{2 \theta_{2}^{*}\left(\theta^{*} A_{0}+\theta_{2} C_{0}\right) \cos ^{2} \frac{1}{2} R t}{R^{2}}+\frac{-2 \theta^{*} \theta_{2}^{*} A_{0}+\left(R^{2}-2\left|\theta_{2}\right|^{2}\right) C_{0}}{R^{2}},
\end{aligned}
$$

$\operatorname{com} R=\sqrt{|\theta|^{2}+\left|\theta_{2}\right|^{2}}$. Então, para $C_{0}=0$ e $R t=\pi$

$$
\begin{aligned}
A & =-A_{0}\left(1-\frac{2\left|\theta_{2}\right|^{2}}{R^{2}}\right), \\
B & =0, \\
C & =\frac{-2 \theta^{*} \theta_{2}^{*} A_{0}}{R^{2}}
\end{aligned}
$$


e haverá completa transferência de populações de $A$ para $C$ fazendo $\theta_{2}=x \theta^{*}$, sendo

$$
x=\left(C_{0} \pm \sqrt{C_{0}^{2}+A_{0}^{2}}\right) / A_{0}= \pm 1 \text {. Assim, ajustando-se }|\theta|=\left|\theta_{2}\right| \text {, pode-se criar o estado vestido }
$$

$\left|\varphi_{4, \mathcal{R}_{2}}\right\rangle$. Outras duas situações podem ser observadas: quando $C_{0}=0\left(A_{0}=1\right)$ e $R t=2 \pi$ o estado retorna ao estado inicial e quando $C_{0}=0$ e $\left|\theta_{2}\right| \gg|\theta|$ obtém-se $A \approx A_{0}(B, C \ll 1)$.

A dinâmica sob a presença da RAP, considerando no máximo quatro excitações, fornece as equações

$$
\begin{aligned}
& \frac{d}{d t} \tilde{\rho}_{0,0}^{-,-}=2\left(\theta \tilde{\rho}_{2,0}^{\mathcal{R},-}\right)^{i}+\frac{\gamma}{2}\left(\tilde{\rho}_{1,1}^{\mathcal{R}, \mathcal{R}}+\tilde{\rho}_{1,1}^{-\mathcal{R},-\mathcal{R}}\right) \\
& \frac{d}{d t}\left(\theta \tilde{\rho}_{2,0}^{\mathcal{R},-}\right)^{i}=-|\theta|^{2} \tilde{\rho}_{0,0}^{-,-}-\left(\theta \theta_{2} \tilde{\rho}_{4,0}^{\mathcal{R}_{2},-}\right)^{r}+|\theta|^{2} \tilde{\rho}_{2,2}^{\mathcal{R}, \mathcal{R}}-\frac{1}{2} \frac{\gamma}{2}\left(\theta \tilde{\rho}_{2,0}^{\mathcal{R},-}\right)^{i} \\
& \frac{d}{d t}\left(\theta \theta_{2} \tilde{\rho}_{4,0}^{\mathcal{R}_{2},-}\right)^{r}=\left|\theta_{2}\right|^{2}\left(\theta \tilde{\rho}_{2,0}^{\mathcal{R},-}\right)^{i}-|\theta|^{2}\left(\theta_{2} \tilde{\rho}_{4,2}^{\mathcal{R}_{2}, \mathcal{R}}\right)^{i}-\frac{1}{2} \frac{\gamma}{2}\left(\theta \theta_{2} \tilde{\rho}_{4,0} \mathcal{R}_{2,-}\right)^{r} \\
& \frac{d}{d t}\left(\theta_{2} \tilde{\rho}_{4,2}^{\mathcal{R}_{2}, \mathcal{R}}\right)^{i}=-\left|\theta_{2}\right|^{2} \tilde{\rho}_{2,2}^{\mathcal{R}, \mathcal{R}}+\left(\theta \theta_{2} \tilde{\rho}_{4,0}^{\mathcal{R}_{2},-}\right)^{r}+\left|\theta_{2}\right|^{2} \tilde{\rho}_{4,4}^{\mathcal{R}_{2}, \mathcal{R}_{2}}-\frac{\gamma}{2}\left(\theta_{2} \tilde{\rho}_{4,2}^{\mathcal{R}_{2}, \mathcal{R}}\right)^{i} \\
& \frac{d}{d t} \tilde{\rho}_{1,1}^{\mathcal{R}, \mathcal{R}}=\frac{\gamma}{2}\left(\frac{1}{2}\left[\tilde{\rho}_{2,2}^{\mathcal{R}, \mathcal{R}}+\tilde{\rho}_{2,2}^{-\mathcal{R},-\mathcal{R}}\right]-\tilde{\rho}_{1,1}^{\mathcal{R}, \mathcal{R}}\right) \\
& \frac{d}{d t} \tilde{\rho}_{1,1}^{-\mathcal{R},-\mathcal{R}}=\frac{\gamma}{2}\left(\frac{1}{2}\left[\tilde{\rho}_{2,2}^{-\mathcal{R},-\mathcal{R}}+\tilde{\rho}_{2,2}^{\mathcal{R}, \mathcal{R}}\right]-\tilde{\rho}_{1,1}^{-\mathcal{R},-\mathcal{R}}\right) \\
& \frac{d}{d t} \tilde{\rho}_{2,2}^{\mathcal{R}, \mathcal{R}}=2\left(\theta_{2} \tilde{\rho}_{4,2}^{\mathcal{R}_{2}, \mathcal{R}}\right)^{i}-2\left(\theta \tilde{\rho}_{2,0}^{\mathcal{R},-}\right)^{i}+\frac{\gamma}{2}\left(\frac{1}{2}\left[\tilde{\rho}_{3,3}^{\mathcal{R}_{2}, \mathcal{R}_{2}}+\tilde{\rho}_{3,3}^{-\mathcal{R}_{2},-\mathcal{R}_{2}}\right]-\tilde{\rho}_{2,2}^{\mathcal{R}, \mathcal{R}}\right) \\
& \frac{d}{d t} \tilde{\rho}_{3,3}^{\mathcal{R}_{2}, \mathcal{R}_{2}}=\frac{\gamma}{2}\left(\frac{1}{2}\left[\tilde{\rho}_{4,4}^{\mathcal{R}_{2}, \mathcal{R}_{2}}+\tilde{\rho}_{4,4}^{-\mathcal{R}_{2},-\mathcal{R}_{2}}\right]-\tilde{\rho}_{3,3}^{\mathcal{R}_{2}, \mathcal{R}_{2}}\right) \\
& \frac{d}{d t} \tilde{\rho}_{3,3}^{-\mathcal{R}_{2},-\mathcal{R}_{2}}=\frac{\gamma}{2}\left(\frac{1}{2}\left[\tilde{\rho}_{4,4}^{-\mathcal{R}_{2},-\mathcal{R}_{2}}+\tilde{\rho}_{4,4}^{\mathcal{R}_{2}, \mathcal{R}_{2}}\right]-\tilde{\rho}_{3,3}^{-\mathcal{R}_{2},-\mathcal{R}_{2}}\right) . \\
& \frac{d}{d t} \tilde{\rho}_{2,2}^{-\mathcal{R},-\mathcal{R}}=\frac{\gamma}{2}\left(\frac{1}{2}\left[\tilde{\rho}_{3,3}^{-\mathcal{R}_{2},-\mathcal{R}_{2}}+\tilde{\rho}_{3,3}^{\mathcal{R}_{2}, \mathcal{R}_{2}}\right]-\tilde{\rho}_{2,2}^{-\mathcal{R},-\mathcal{R}}\right) \\
& \frac{d}{d t} \tilde{\rho}_{4,4}^{\mathcal{R}_{2}, \mathcal{R}_{2}}=-2\left(\theta_{2} \tilde{\rho}_{4,2}^{\mathcal{R}_{2}, \mathcal{R}}\right)^{i}-\frac{\gamma}{2} \tilde{\rho}_{4,4}^{\mathcal{R}_{2}, \mathcal{R}_{2}} \\
& \frac{d}{d t} \tilde{\rho}_{4,4}^{-\mathcal{R}_{2},-\mathcal{R}_{2}}=-\frac{\gamma}{2} \tilde{\rho}_{4,4}^{-\mathcal{R}_{2},-\mathcal{R}_{2}}
\end{aligned}
$$

$\mathrm{Na}$ evolução unitária pode ocorrer a transferência completa de populações entre $\tilde{\rho}_{0,0}^{-,-}$e $\tilde{\rho}_{4,4}^{\mathcal{R}_{2}, \mathcal{R}_{2}}$ se $|\theta|=\left|\theta_{2}\right|$. Já no caso com dissipação obtém-se para estes parâmetros a solução assintótica

$$
\tilde{\rho}_{0,0}^{-,-}=\left(\frac{\left[2|\theta|^{2}+(\gamma / 4)^{2}\right]}{|\theta|^{2}}-2 \frac{\left[\frac{1}{2}|\theta|^{2}-\frac{1}{2}(\gamma / 4)^{2}\right]}{\left(\frac{3}{2}|\theta|^{2}+\frac{\gamma^{2}}{8}\right)}\right) \tilde{\rho}_{2,2}^{\mathcal{R}, \mathcal{R}}
$$




$$
\tilde{\rho}_{4,4}^{\mathcal{R}_{2}, \mathcal{R}_{2}}=\frac{2|\theta|^{2}}{\left(\frac{3}{2}|\theta|^{2}+\frac{\gamma^{2}}{8}\right)} \tilde{\rho}_{2,2}^{\mathcal{R}, \mathcal{R}} .
$$

A condição $\gamma \ll|\theta|$ mostra que as populações tem como resultados aproximados

$$
\begin{gathered}
\tilde{\rho}_{2,2}^{\mathcal{R}, \mathcal{R}} \approx \frac{3}{4} \tilde{\rho}_{0,0}^{-,-}, \\
\tilde{\rho}_{4,4}^{\mathcal{R}_{2}, \mathcal{R}_{2}} \approx \tilde{\rho}_{0,0}^{-,-}, \\
\tilde{\rho}_{4,4}^{-\mathcal{R}_{2},-\mathcal{R}_{2}}=0, \\
\tilde{\rho}_{1,1}^{\mathcal{R}, \mathcal{R}}=\tilde{\rho}_{1,1}^{-\mathcal{R},-\mathcal{R}}=\frac{5}{8} \tilde{\rho}_{0,0}^{-,-}, \\
\tilde{\rho}_{3,3}^{\mathcal{R}_{2}, \mathcal{R}_{2}}=\tilde{\rho}_{3,3}^{-\mathcal{R}_{2},-\mathcal{R}_{2}}=\tilde{\rho}_{2,2}^{-\mathcal{R},-\mathcal{R}}=\frac{1}{2} \tilde{\rho}_{0,0}^{-,-} .
\end{gathered}
$$

Logo, a condição de normalização nos fornece

$$
\tilde{\rho}_{0,0}^{-,-}=\frac{2}{11},
$$

o que demonstra que o estado atingido assintoticamente possui uma probabilidade bastante pequena de ter zero excitações.

$$
\begin{gathered}
\text { Para a RPC, as equações para o caso } \mathcal{R}=\mathcal{R}_{2} \text { são } \\
\frac{d}{d t} \tilde{\rho}_{0,0}^{-,-}=2\left(\theta \tilde{\rho}_{2,0}^{\mathcal{R},-}\right)^{i}+\frac{\kappa}{2}\left(\tilde{\rho}_{1,1}^{\mathcal{R} \mathcal{R}}+\tilde{\rho}_{1,1}^{-\mathcal{R},-\mathcal{R}}\right) \\
\frac{d}{d t}\left(\theta \tilde{\rho}_{2,0}^{\mathcal{R},-}\right)^{i}=-|\theta|^{2} \tilde{\rho}_{0,0}^{-,-}-\left(\theta \theta_{2} \tilde{\rho}_{4,0}^{\mathcal{R},-}\right)^{r}+|\theta|^{2} \tilde{\rho}_{2,2}^{\mathcal{R}, \mathcal{R}}-\frac{\kappa}{2} \frac{3}{2}\left(\theta \tilde{\rho}_{2,0}^{\mathcal{R},-}\right)^{i} \\
\frac{d}{d t}\left(\theta \theta_{2} \tilde{\rho}_{4,0}^{\mathcal{R},-}\right)^{r}=\left|\theta_{2}\right|^{2}\left(\theta \tilde{\rho}_{2,0}^{\mathcal{R},-}\right)^{i}-|\theta|^{2}\left(\theta_{2} \tilde{\rho}_{4,2}^{\mathcal{R}, \mathcal{R}}\right)^{i}-\frac{\kappa}{2} \frac{7}{2}\left(\theta \theta_{2} \tilde{\rho}_{4,0}^{\mathcal{R},-}\right)^{r} \\
\frac{d}{d t}\left(\theta_{2} \tilde{\rho}_{4,2}^{\mathcal{R}, \mathcal{R}}\right)^{i}=-\left|\theta_{2}\right|^{2} \tilde{\rho}_{2,2}^{\mathcal{R}, \mathcal{R}}+\left(\theta \theta_{2} \tilde{\rho}_{4,0}^{\mathcal{R},-}\right)^{r}+\left|\theta_{2}\right|^{2} \tilde{\rho}_{4,4}^{\mathcal{R}, \mathcal{R}}-\frac{\kappa}{2} 5\left(\theta_{2} \tilde{\rho}_{4,2}^{\mathcal{R}, \mathcal{R}}\right)^{i} \\
\frac{d}{d t} \tilde{\rho}_{1,1}^{\mathcal{R}, \mathcal{R}}=\frac{\kappa}{2}\left(\frac{1}{2}\left[(\sqrt{2}+1)^{2} \tilde{\rho}_{2,2}^{\mathcal{R}, \mathcal{R}}+(\sqrt{2}-1)^{2} \tilde{\rho}_{2,2}^{-\mathcal{R},-\mathcal{R}}\right]-\tilde{\rho}_{1,1}^{\mathcal{R}, \mathcal{R}}\right) \\
\frac{d}{d t} \tilde{\rho}_{1,1}^{-\mathcal{R},-\mathcal{R}}=\frac{\kappa}{2}\left(\frac{1}{2}\left[(\sqrt{2}+1)^{2} \tilde{\rho}_{2,2}^{-\mathcal{R},-\mathcal{R}}+(\sqrt{2}-1)^{2} \tilde{\rho}_{2,2}^{\mathcal{R}, \mathcal{R}}\right]-\tilde{\rho}_{1,1}^{-\mathcal{R},-\mathcal{R}}\right) \\
\frac{d}{d t} \tilde{\rho}_{2,2}^{\mathcal{R}, \mathcal{R}}=2\left(\theta_{2} \tilde{\rho}_{4,2}^{\mathcal{R}, \mathcal{R}}\right)^{i}-2\left(\theta \tilde{\rho}_{2,0}^{\mathcal{R},-}\right)^{i}+\frac{\kappa}{2}\left(\frac{1}{2}\left[(\sqrt{3}+\sqrt{2})^{2} \tilde{\rho}_{3,3}^{\mathcal{R}, \mathcal{R}}+(\sqrt{3}-\sqrt{2})^{2} \tilde{\rho}_{3,3}^{-\mathcal{R},-\mathcal{R}}\right]-3 \tilde{\rho}_{2,2}^{\mathcal{R}, \mathcal{R}}\right) \\
\frac{d}{d t} \tilde{\rho}_{2,2}^{-\mathcal{R},-\mathcal{R}}=\frac{\kappa}{2}\left(\frac{1}{2}\left[(\sqrt{3}+\sqrt{2})^{2} \tilde{\rho}_{3,3}^{-\mathcal{R},-\mathcal{R}}+(\sqrt{3}-\sqrt{2})^{2} \tilde{\rho}_{3,3}^{\mathcal{R}, \mathcal{R}}\right]-3 \tilde{\rho}_{2,2}^{-\mathcal{R},-\mathcal{R}}\right)
\end{gathered}
$$




$$
\begin{gathered}
\frac{d}{d t} \tilde{\rho}_{3,3}^{\mathcal{R}, \mathcal{R}}=\frac{\kappa}{2}\left(\frac{1}{2}\left[(2+\sqrt{3})^{2} \tilde{\rho}_{4,4}^{\mathcal{R}, \mathcal{R}}+(2-\sqrt{3})^{2} \tilde{\rho}_{4,4}^{-\mathcal{R},-\mathcal{R}}\right]-5 \tilde{\rho}_{3,3}^{\mathcal{R}, \mathcal{R}}\right) \\
\frac{d}{d t} \tilde{\rho}_{3,3}^{-\mathcal{R},-\mathcal{R}}=\frac{\kappa}{2}\left(\frac{1}{2}\left[(2+\sqrt{3})^{2} \tilde{\rho}_{4,4}^{-\mathcal{R},-\mathcal{R}}+(2-\sqrt{3})^{2} \tilde{\rho}_{4,4}^{\mathcal{R}, \mathcal{R}}\right]-5 \tilde{\rho}_{3,3}^{-\mathcal{R},-\mathcal{R}}\right) \\
\frac{d}{d t} \tilde{\rho}_{4,4}^{\mathcal{R}, \mathcal{R}}=-2\left(\theta_{2} \tilde{\rho}_{4,2}^{\mathcal{R}, \mathcal{R}}\right)^{i}-7 \frac{\kappa}{2} \tilde{\rho}_{4,4}^{\mathcal{R}, \mathcal{R}} \\
\frac{d}{d t} \tilde{\rho}_{4,4}^{-\mathcal{R},-\mathcal{R}}=-7 \frac{\kappa}{2} \tilde{\rho}_{4,4}^{-\mathcal{R},-\mathcal{R}} .
\end{gathered}
$$

E a solução assintótica junto à condição de normalização fornece probabilidades

$$
\begin{gathered}
\tilde{\rho}_{1,1}^{-\mathcal{R},-\mathcal{R}} \approx 0,22 \tilde{\rho}_{0,0}^{-,-}, \\
\tilde{\rho}_{1,1}^{\mathcal{R}, \mathcal{R}} \approx 4,05 \tilde{\rho}_{0,0}^{-,-}, \\
\tilde{\rho}_{2,2}^{\mathcal{R}, \mathcal{R}} \approx 1,39 \tilde{\rho}_{0,0}^{-,-}, \\
\tilde{\rho}_{2,2}^{-\mathcal{R},-\mathcal{R}} \approx 3,53 \times 10^{-2} \tilde{\rho}_{0,0}^{-,-}, \\
\tilde{\rho}_{3,3}^{\mathcal{R}, \mathcal{R}} \approx 1,39 \tilde{\rho}_{0,0}^{-,-}, \\
\tilde{\rho}_{3,3}^{-\mathcal{R},-\mathcal{R}} \approx 7,18 \times 10^{-3} \tilde{\rho}_{0,0}^{-,-}, \\
\tilde{\rho}_{4,4}^{\mathcal{R}, \mathcal{R}} \approx \tilde{\rho}_{0,0}^{-,-}=0,11 .
\end{gathered}
$$

quando $\left|\theta_{2}\right|=|\theta| \gg \kappa$. Se se considerar $\mathcal{R}_{2}=-\mathcal{R}$, a solução assintótica fornecerá como probabilidades não nulas

$$
\begin{gathered}
\tilde{\rho}_{1,1}^{-\mathcal{R},-\mathcal{R}} \approx 6,76 \tilde{\rho}_{0,0}^{-,-}, \\
\tilde{\rho}_{1,1}^{\mathcal{R}, \mathcal{R}} \approx 2,26 \tilde{\rho}_{0,0}^{-,-}, \\
\tilde{\rho}_{2,2}^{-\mathcal{R},-\mathcal{R}} \approx 2,23 \tilde{\rho}_{0,0}^{-,-}, \\
\tilde{\rho}_{2,2}^{\mathcal{R}, \mathcal{R}} \approx 0.71 \tilde{\rho}_{0,0}^{-,-}, \\
\tilde{\rho}_{3,3}^{\mathcal{R}, \mathcal{R}} \approx 7,18 \times 10^{-3} \tilde{\rho}_{0,0}^{-,-}, \\
\tilde{\rho}_{3,3}^{-\mathcal{R},-\mathcal{R}} \approx 1,39 \tilde{\rho}_{0,0}^{-,-}, \\
\tilde{\rho}_{4,4}^{-\mathcal{R},-\mathcal{R}} \approx \tilde{\rho}_{0,0}^{-,-}=0,06 .
\end{gathered}
$$


A dinâmica, após considerarmos no máximo quatro excitações e eliminarmos termos de rápida oscilação por RWA, sob a DAP obtida das equações (4.23), (4.24) e (4.25) fornece as seguintes equações

$$
\begin{gathered}
\frac{d}{d t} \tilde{\rho}_{0,0}^{-,-}=2\left(\theta \tilde{\rho}_{2,0}^{\mathcal{R},-}\right)^{i} \\
\frac{d}{d t}\left(\theta \tilde{\rho}_{2,0}^{\mathcal{R},-}\right)^{i}=-|\theta|^{2} \tilde{\rho}_{0,0}^{-,-}-\left(\theta \theta_{2} \tilde{\rho}_{4,0}^{\mathcal{R}_{2,-}}\right)^{r}+|\theta|^{2} \tilde{\rho}_{2,2}^{\mathcal{R}, \mathcal{R}}-\frac{\gamma_{\phi}}{2}\left(\theta \tilde{\rho}_{2,0}^{\mathcal{R},-}\right)^{i} \\
\frac{d}{d t}\left(\theta \theta_{2} \tilde{\rho}_{4,0}^{\mathcal{R}_{2},-}\right)^{r}=\left|\theta_{2}\right|^{2}\left(\theta \tilde{\rho}_{2,0}^{\mathcal{R},-}\right)^{i}-|\theta|^{2}\left(\theta_{2} \tilde{\rho}_{4,2}^{\mathcal{R}_{2}, \mathcal{R}}\right)^{i}-\frac{\gamma_{\phi}}{2}\left(\theta \theta_{2} \tilde{\rho}_{4,0}^{\mathcal{R}_{2},-}\right)^{r}, \\
\frac{d}{d t}\left(\theta_{2} \tilde{\rho}_{4,2}^{\mathcal{R}_{2}, \mathcal{R}}\right)^{i}=-\left|\theta_{2}\right|^{2} \tilde{\rho}_{2,2}^{\mathcal{R}, \mathcal{R}}+\left(\theta \theta_{2} \tilde{\rho}_{4,0}^{\mathcal{R}_{2},-}\right)^{r}+\left|\theta_{2}\right|^{2} \tilde{\rho}_{4,4}^{\mathcal{R}_{2}, \mathcal{R}_{2}}-\frac{\gamma_{\phi}}{2}\left(\theta_{2} \tilde{\rho}_{4,2}^{\mathcal{R}_{2}, \mathcal{R}}\right)^{i}, \\
\frac{d}{d t} \tilde{\rho}_{2,2}^{\mathcal{R}, \mathcal{R}}=2\left(\theta_{2} \tilde{\rho}_{4,2}^{\mathcal{R}_{2}, \mathcal{R}}\right)^{i}-2\left(\theta \tilde{\rho}_{2,-}^{\mathcal{R},-}\right)^{i}+\frac{\gamma_{\phi}}{2}\left[-\tilde{\rho}_{2,2}^{\mathcal{R}, \mathcal{R}}+\tilde{\rho}_{2,2}^{-\mathcal{R},-\mathcal{R}}\right], \\
\frac{d}{d t} \tilde{\rho}_{2,2}^{-\mathcal{R},-\mathcal{R}}=\frac{\gamma_{\phi}}{2}\left[-\tilde{\rho}_{2,2}^{-\mathcal{R},-\mathcal{R}}+\tilde{\rho}_{2,2}^{\mathcal{R}, \mathcal{R}}\right] \\
\frac{d}{d t} \tilde{\rho}_{4,4}^{\mathcal{R}_{2}, \mathcal{R}_{2}}=-2\left(\theta_{2} \tilde{\rho}_{4,2}^{\mathcal{R}_{2}, \mathcal{R}}\right)^{i}+\frac{\gamma_{\phi}}{2}\left[-\tilde{\rho}_{4,4}^{\mathcal{R}_{2}, \mathcal{R}_{2}}+\tilde{\rho}_{4,4}^{-\mathcal{R}_{2},-\mathcal{R}_{2}}\right], \\
\frac{d}{d t} \tilde{\rho}_{4,4}^{-\mathcal{R}_{2},-\mathcal{R}_{2}}=\frac{\gamma_{\phi}}{2}\left[-\tilde{\rho}_{4,4}^{-\mathcal{R}_{2,-} \mathcal{R}_{2}}+\tilde{\rho}_{4,4}^{\mathcal{R}_{2}, \mathcal{R}_{2}}\right] .
\end{gathered}
$$

No limite assintótico as probabilidades não nulas, obtidas pela normalização, são

$$
\tilde{\rho}_{0,0}^{-,-}=\tilde{\rho}_{2,2}^{-\mathcal{R},-\mathcal{R}}=\tilde{\rho}_{2,2}^{\mathcal{R}, \mathcal{R}}=\tilde{\rho}_{4,4}^{-\mathcal{R}_{2},-\mathcal{R}_{2}}=\tilde{\rho}_{4,4}^{\mathcal{R}_{2}, \mathcal{R}_{2}}=\frac{1}{5} .
$$

Concluindo, para taxas $\gamma, \kappa, \gamma_{\phi} \ll|\theta|$ quatro excitações podem ser geradas do vácuo.

\subsubsection{Regime anti-ECD incrementado}

O comportamento anti-ECD pode ser incrementado combinando à sua frequência de modulação a frequência modulada $\eta_{k}^{(A 2)}=\Delta_{+}-2\left(\delta_{-}-\delta_{+}\right)(k-3), k \geq 4$. O hamiltoniano efetivo é, então,

$$
\begin{aligned}
\tilde{H} & =\sum_{m, \mathcal{S}, \mathcal{T}} \sum_{\eta_{k}^{(A)}, \eta_{k}^{(A 2)}} \Xi_{m, \mathcal{T}, \mathcal{S}}^{(j)} e^{-i t\left(\lambda_{m+2, \mathcal{S}}-\lambda_{m, \mathcal{T}}-\eta^{(j)}\right)}\left|\varphi_{m, \mathcal{T}}\right\rangle\left\langle\varphi_{m+2, \mathcal{S}}\right|+h . c . \\
& =\left(\theta^{*}\left|\varphi_{k, \mathcal{D}}\right\rangle\left\langle\varphi_{k-2,-\mathcal{D}}|+\theta| \varphi_{k-2,-\mathcal{D}}\right\rangle\left\langle\varphi_{k, \mathcal{D}}\left|+\theta_{2}^{*}\right| \varphi_{k-2,-\mathcal{D}}\right\rangle\left\langle\varphi_{k-4, \mathcal{D}}\left|+\theta_{2}\right| \varphi_{k-4, \mathcal{D}}\right\rangle\left\langle\varphi_{k-2,-\mathcal{D}}\right|\right),
\end{aligned}
$$

$\operatorname{com} \theta$ permanecendo o mesmo para o regime anti-ECD e

$$
\begin{aligned}
\theta_{2} & =i \frac{1}{2} g_{0} \mathcal{D} \sqrt{k-3} \Upsilon^{(A 2)}, \\
\Upsilon^{(A 2)} & =\left[-\frac{\varepsilon_{\omega}^{(A 2)}}{\Delta_{+}}-\frac{\varepsilon_{\Omega}^{(A 2)}}{\Delta_{+}}+\frac{\varepsilon_{g}^{(A 2)}}{g_{0}}+i \frac{2 \varepsilon_{\chi}^{(A 2)}}{\Delta_{-}}\right] .
\end{aligned}
$$


Nesse regime combina-se simultaneamente o acoplamento $\left|\varphi_{k, \mathcal{D}}\right\rangle \rightarrow\left|\varphi_{k-2,-\mathcal{D}}\right\rangle$ via anti-ECD com o acoplamento $\left|\varphi_{k-2,-\mathcal{D}}\right\rangle \rightarrow\left|\varphi_{k-4, \mathcal{D}}\right\rangle$ via comportamento AJC, obtendo a aniquilação de quatro excitações a partir do estado $|g, k\rangle\langle g, k|$ no RD. Quando $k=4$, em um caso específico, o estado inicial $|g, 4\rangle$ vai ao EZE $|g, 0\rangle$. Importante observar que o Hamiltoniano efetivo é semelhante em estrutura ao obtido em (5.98), então as equações (5.107)-(5.109) podem ser aplicadas com as substituições apropriadas para estimar o coeficiente $\theta_{2}$. Se o estado inicial da cavidade é conhecido e a população do estado atômico excitado é desprezível, pode-se transferir as populações de $\left|\varphi_{k, \mathcal{D}}\right\rangle$ para $\left|\varphi_{k-4, \mathcal{D}}\right\rangle$.

A DU é descrita pelas equações

$$
\begin{gathered}
\frac{d}{d t} \tilde{\rho}_{k, k}^{\mathcal{D}, \mathcal{D}}=2\left(\theta \tilde{\rho}_{k-2, k}^{-\mathcal{D}, \mathcal{D}}\right)^{i}, \\
\frac{d}{d t}\left(\theta \tilde{\rho}_{k-2, k}^{-\mathcal{D}, \mathcal{D}}\right)^{i}=-|\theta|^{2} \tilde{\rho}_{k, k}^{\mathcal{D}, \mathcal{D}}+|\theta|^{2} \tilde{\rho}_{k-2, k-2}^{-\mathcal{D},-\mathcal{D}}-\left(\theta \theta_{2} \tilde{\rho}_{k-4, k}^{\mathcal{D}, \mathcal{D}}\right)^{r}, \\
\frac{d}{d t}\left(\theta \theta_{2} \tilde{\rho}_{k-4, k}^{\mathcal{D}, \mathcal{D}}\right)^{r}=\left|\theta_{2}\right|^{2}\left(\theta \tilde{\rho}_{k-2, k}^{-\mathcal{D}, \mathcal{D}}\right)^{i}-|\theta|^{2}\left(\theta_{2} \tilde{\rho}_{k-4, k-2}^{\mathcal{D},-\mathcal{D}}\right)^{i}, \\
\frac{d}{d t}\left(\theta_{2} \tilde{\rho}_{k-4, k-2}^{\mathcal{D},-\mathcal{D}}\right)^{i}=-\left|\theta_{2}\right|^{2} \tilde{\rho}_{k-2, k-2}^{-\mathcal{D},-\mathcal{D}}+\left(\theta \theta_{2} \tilde{\rho}_{k-4, k}^{\mathcal{D}, \mathcal{D}}\right)^{r}+\left|\theta_{2}\right|^{2} \tilde{\rho}_{k-4, k-4}^{\mathcal{D}, \mathcal{D}}, \\
\frac{d}{d t} \tilde{\rho}_{k-2, k-2}^{-\mathcal{D},-\mathcal{D}}=-2\left(\theta \tilde{\rho}_{k-2, k}^{-\mathcal{D}, \mathcal{D}}\right)^{i}+2\left(\theta_{2} \tilde{\rho}_{k-4, k-2}^{\mathcal{D},-\mathcal{D}}\right)^{i}, \\
\frac{d}{d t} \tilde{\rho}_{k-4, k-4}^{\mathcal{D}, \mathcal{D}}=-2\left(\theta_{2} \tilde{\rho}_{k-4, k-2}^{\mathcal{D},-\mathcal{D}}\right)^{i},
\end{gathered}
$$

de forma que serão analisadas as influências da DAP e RAP. Quando a DU está sob DAP, considerandose um estado inicial $\tilde{\rho}_{k, k}^{\mathcal{D}, \mathcal{D}}$, as equações obtidas serão

$$
\begin{gathered}
\frac{d}{d t} \tilde{\rho}_{k, k}^{\mathcal{D}, \mathcal{D}}=2\left(\theta \tilde{\rho}_{k-2, k}^{-\mathcal{D}, \mathcal{D}}\right)^{i}+2 k \gamma_{\phi} \frac{g_{0}^{2}}{\Delta_{-}^{2}}\left[-\tilde{\rho}_{k, k}^{\mathcal{D}, \mathcal{D}}+\tilde{\rho}_{k, k}^{-\mathcal{D},-\mathcal{D}}\right], \\
\frac{d}{d t} \tilde{\rho}_{k, k}^{-\mathcal{D},-\mathcal{D}}=2 k \gamma_{\phi} \frac{g_{0}^{2}}{\Delta_{-}^{2}}\left[-\tilde{\rho}_{k, k}^{-\mathcal{D},-\mathcal{D}}+\tilde{\rho}_{k, k}^{\mathcal{D}, \mathcal{D}}\right], \\
\frac{d}{d t}\left(\theta \tilde{\rho}_{k-2, k}^{-\mathcal{D}, \mathcal{D}}\right)^{i}=-|\theta|^{2} \tilde{\rho}_{k, k}^{\mathcal{D}, \mathcal{D}}+|\theta|^{2} \tilde{\rho}_{k-2, k-2}^{-\mathcal{D},-\mathcal{D}}-\left(\theta \theta_{2} \tilde{\rho}_{k-4, k}^{\mathcal{D}, \mathcal{D}}\right)^{r} \\
\left.\frac{d}{d t}\left(\theta \theta_{2} \tilde{\rho}_{k-4, k}^{\mathcal{D}, \mathcal{D}}\right)^{r}=\left|\theta_{2}\right|^{2}\left(\theta \tilde{\rho}_{k-2, k}^{-\mathcal{D}, \mathcal{D}}\right)^{i}-|\theta|^{2}\left(\theta_{2} \tilde{\rho}_{k-4, k-2}^{\mathcal{D},-\mathcal{D}}\right)^{i}-\gamma_{\phi} \frac{g_{0}^{2}}{\Delta_{-}^{2}} 2(k-1)\right)\left(\theta \tilde{\rho}_{k-2, k}^{-}{ }_{-}^{2}, \mathcal{D}\right)^{i},
\end{gathered}
$$




$$
\begin{aligned}
& \frac{d}{d t}\left(\theta_{2} \tilde{\rho}_{k-4, k-2}^{\mathcal{D},-\mathcal{D}}\right)^{i}=-\left|\theta_{2}\right|^{2} \tilde{\rho}_{k-2, k-2}^{-\mathcal{D},-\mathcal{D}}+\left(\theta \theta_{2} \tilde{\rho}_{k-4, k}^{\mathcal{D}, \mathcal{D}}\right)^{r}+\left|\theta_{2}\right|^{2} \tilde{\rho}_{k-4, k-4}^{\mathcal{D}, \mathcal{D}} \\
& -\gamma_{\phi}\left(1-\frac{g_{0}^{2}}{\Delta_{-}^{2}} 2(k-3)\right)\left(\theta_{2} \tilde{\rho}_{k-4, k-2}^{\mathcal{D},-\mathcal{D}}\right)^{i} \\
& \frac{d}{d t} \tilde{\rho}_{k-2, k-2}^{-\mathcal{D},-\mathcal{D}}=-2\left(\theta \tilde{\rho}_{k-2, k}^{-\mathcal{D}, \mathcal{D}}\right)^{i}+2\left(\theta_{2} \tilde{\rho}_{k-4, k-2}^{\mathcal{D},-\mathcal{D}}\right)^{i}+2(k-2) \gamma_{\phi} \frac{g_{0}^{2}}{\Delta_{0}^{2}}\left[-\tilde{\rho}_{k-2, k-2}^{-\mathcal{D},-\mathcal{D}}+\tilde{\rho}_{k-2, k-2}^{\mathcal{D}, \mathcal{D}}\right] \\
& \frac{d}{d t} \tilde{\rho}_{k-2, k-2}^{\mathcal{D}, \mathcal{D}}=2(k-2) \gamma_{\phi} \frac{g_{0}^{2}}{\Delta_{-}^{2}}\left[-\tilde{\rho}_{k-2, k-2}^{\mathcal{D}, \mathcal{D}}+\tilde{\rho}_{k-2, k-2}^{-\mathcal{D},-\mathcal{D}}\right], \\
& \frac{d}{d t} \tilde{\rho}_{k-4, k-4}^{\mathcal{D}, \mathcal{D}}=-2\left(\theta_{2} \tilde{\rho}_{k-4, k-2}^{\mathcal{D},-\mathcal{D}}\right)^{i}+2(k-4) \gamma_{\phi} \frac{g_{0}^{2}}{\Delta_{-}^{2}}\left[-\tilde{\rho}_{k-4, k-4}^{\mathcal{D}, \mathcal{D}}+\tilde{\rho}_{k-4, k-4}^{-\mathcal{D},-\mathcal{D}}\right] \\
& \frac{d}{d t} \tilde{\rho}_{k-4, k-4}^{-\mathcal{D},-\mathcal{D}}=2(k-4) \gamma_{\phi} \frac{g_{0}^{2}}{\Delta_{-}^{2}}\left[-\tilde{\rho}_{k-4, k-4}^{-\mathcal{D},-\mathcal{D}}+\tilde{\rho}_{k-4, k-4}^{\mathcal{D}, \mathcal{D}}\right]
\end{aligned}
$$

sendo sua solução assintótica quando $k=4$

$$
\begin{gathered}
\tilde{\rho}_{4,4}^{\mathcal{D}, \mathcal{D}}=\tilde{\rho}_{4,4}^{-\mathcal{D},-\mathcal{D}}=\tilde{\rho}_{2,2}^{-\mathcal{D},-\mathcal{D}}=\tilde{\rho}_{2,2}^{\mathcal{D}, \mathcal{D}}=\tilde{\rho}_{0,0}^{-,-}, \\
\tilde{\rho}_{l, l}^{\mathcal{D}, \mathcal{D}}=\tilde{\rho}_{l, l}^{-\mathcal{D},-\mathcal{D}} \text { para qualquer } l .
\end{gathered}
$$

Assim, a DU é drasticamente afetada. Se $k>4$, a solução assintótica diz que

$$
\begin{gathered}
\tilde{\rho}_{k, k}^{-\mathcal{D},-\mathcal{D}}=\tilde{\rho}_{k, k}^{\mathcal{D}, \mathcal{D}}=\tilde{\rho}_{k-2, k-2}^{-\mathcal{D},-\mathcal{D}}=\tilde{\rho}_{k-2, k-2}^{\mathcal{D}, \mathcal{D}}=\tilde{\rho}_{k-4, k-4}^{-\mathcal{D},-\mathcal{D}}=\tilde{\rho}_{k-4, k-4}^{\mathcal{D}, \mathcal{D}} \\
\tilde{\rho}_{l, l}^{\mathcal{D}, \mathcal{D}}=\tilde{\rho}_{l, l}^{-\mathcal{D},-\mathcal{D}} \text { para qualquer } l .
\end{gathered}
$$

Analisando a dinâmica unitária sob RAP, com o auxilio de (4.20), (4.21) e (4.22) obtém-se, com aproximações em primeira ordem em $g_{0} / \Delta_{-}$, as seguintes equações

$$
\begin{gathered}
\frac{d}{d t} \tilde{\rho}_{k+1, k+1}^{-\mathcal{D},-\mathcal{D}}=-\gamma \tilde{\rho}_{k+1, k+1}^{-\mathcal{D},-\mathcal{D}} ; \\
\frac{d}{d t} \tilde{\rho}_{k, k}^{\mathcal{D}, \mathcal{D}}=2\left(\theta \tilde{\rho}_{k-2, k}^{-\mathcal{D}, \mathcal{D}}\right)^{i}+\gamma \tilde{\rho}_{k+1, k+1}^{-\mathcal{D},-\mathcal{D}} ; \\
\frac{d}{d t}\left(\theta \tilde{\rho}_{k-\mathcal{D}, \mathcal{D}}\right)^{i}=-|\theta|^{2} \tilde{\rho}_{k, k}^{\mathcal{D}, \mathcal{D}}+|\theta|^{2} \tilde{\rho}_{k-2, k-2}^{-\mathcal{D},-\mathcal{D}}-\left(\theta \theta_{2} \tilde{\rho}_{k-4, k}^{\mathcal{D}, \mathcal{D}}\right)^{r}-\frac{\gamma}{2}\left(\theta \tilde{\rho}_{k-2, k}^{-\mathcal{D}, \mathcal{D}}\right)^{i} ; \\
\frac{d}{d t}\left(\theta \theta_{2} \tilde{\rho}_{k-4, k}^{\mathcal{D}, \mathcal{D}}\right)^{r}=\left|\theta_{2}\right|^{2}\left(\theta \tilde{\rho}_{k-2, k}^{-\mathcal{D}, \mathcal{D}}\right)^{i}-|\theta|^{2}\left(\theta_{2} \tilde{\rho}_{k-4, k-2}^{\mathcal{D},-\mathcal{D}}\right)^{i}-\frac{\gamma}{2} \frac{g_{0}^{2}}{\Delta_{-}^{2}}(2 k-4)\left(\theta \theta_{2} \tilde{\rho}_{k-4, \mathcal{D}}^{\mathcal{D}, \mathcal{D}}\right)^{r} ; \\
\frac{d}{d t}\left(\theta_{2} \tilde{\rho}_{k-4, k-2}^{\mathcal{D},-\mathcal{D}}\right)^{i}=-\left|\theta_{2}\right|^{2} \tilde{\rho}_{k-2, k-2}^{-\mathcal{D},-\mathcal{D}}+\left(\theta \theta_{2} \tilde{\rho}_{k-4, k}^{\mathcal{D}, \mathcal{D}}\right)^{r}+\left|\theta_{2}\right|^{2} \tilde{\rho}_{k-4, k-4}^{\mathcal{D}, \mathcal{D}}-\frac{\gamma}{2}\left(\theta_{2} \tilde{\rho}_{k-4, k-2}^{\mathcal{D},-\mathcal{D}}\right)^{i} ;
\end{gathered}
$$




$$
\begin{gathered}
\frac{d}{d t} \tilde{\rho}_{k-1, k-1}^{-\mathcal{D},-\mathcal{D}}=-\gamma \tilde{\rho}_{k-1, k-1}^{-\mathcal{D},-\mathcal{D}} ; \\
\frac{d}{d t} \tilde{\rho}_{k-2, k-2}^{\mathcal{D}, \mathcal{D}}=\gamma \tilde{\rho}_{k-1, k-1}^{-\mathcal{D},-\mathcal{D}} ; \\
\frac{d}{d t} \tilde{\rho}_{k-2, k-2}^{-\mathcal{D},-\mathcal{D}}=-2\left(\theta \tilde{\rho}_{k-2, k}^{-\mathcal{D}, \mathcal{D}}\right)^{i}+2\left(\theta_{2} \tilde{\rho}_{k-4, k-2}^{\mathcal{D},-\mathcal{D}}\right)^{i}-\gamma \tilde{\rho}_{k-2, k-2}^{-\mathcal{D},-\mathcal{D}} ; \\
\frac{d}{d t} \tilde{\rho}_{k-3, k-3}^{-\mathcal{D},-\mathcal{D}}=-\gamma \tilde{\rho}_{k-3, k-3}^{-\mathcal{D},-\mathcal{D}} ; \\
\frac{d}{d t} \tilde{\rho}_{k-4, k-4}^{\mathcal{D}, \mathcal{D}}=-2\left(\theta_{2} \tilde{\rho}_{k-4, k-2}^{\mathcal{D},-\mathcal{D}}\right)^{i}+\gamma \tilde{\rho}_{k-3, k-3}^{-\mathcal{D},-\mathcal{D}} ; \\
\frac{d}{d t} \tilde{\rho}_{k-4, k-4}^{-\mathcal{D},-\mathcal{D}}=-\gamma \tilde{\rho}_{k-4, k-4}^{-\mathcal{D},-\mathcal{D}} .
\end{gathered}
$$

A análise da solução em primeira ordem prediz que para qualquer $k \geq 4$ a solução assintótica é que apenas $\tilde{\rho}_{k-3, k-3}^{\mathcal{D}, \mathcal{D}}$ é não-nulo e, portanto, igual a 1 pela normalização. Se a solução for tomada em ordem superior em $\frac{g_{0}}{\Delta_{-}}$, a população $\tilde{\rho}_{k-3, k-3}^{\mathcal{D}, \mathcal{D}}$ também decai indo assintóticamente para $\tilde{\rho}_{0,0}^{\mathcal{D}, \mathcal{D}}$.

\subsection{Efeito Casimir Dinâmico fundamental (ECDg)}

Para uma simples modulação fazendo a ressonância do $\operatorname{ECD} \eta^{(D)} \approx 2 \omega_{0}$, a amplitude de probabilidade obedece à equação diferencial $[42,49]$

$$
\begin{aligned}
\dot{b}_{m, \mathcal{T}}(t)= & \sum_{\mathcal{S}}\left[\Theta_{m+2, \mathcal{T}, \mathcal{S}} e^{-i t\left(\tilde{\lambda}_{m+2, \mathcal{S}}-\tilde{\lambda}_{m, \mathcal{T}}-\eta^{(D)}\right.}\right) b_{m+2, \mathcal{S}}(t) \\
& \left.-\Theta_{m, \mathcal{S}, \mathcal{T}}^{*} e^{i t\left(\tilde{\lambda}_{m, \mathcal{T}}-\tilde{\lambda}_{m-2, \mathcal{S}}-\eta^{(D)}\right)} b_{m-2, \mathcal{S}}(t)\right] .
\end{aligned}
$$

A frequência $\eta^{(D)}$ corresponde às diferenças $\tilde{\lambda}_{m+2, \mathcal{S}}-\tilde{\lambda}_{m, \mathcal{T}}$ somente no RD $\left|\Delta_{-}\right| / 2 \gg g_{0} \sqrt{n}$. Além disso, foi demonstrado que a frequência efetiva da cavidade pode ser

$$
\begin{aligned}
& \omega_{g}=\omega_{0}+\delta_{-}-\delta_{+}-\delta_{\chi}, \\
& \omega_{e}=\omega_{0}-\delta_{-}+\delta_{+}-\delta_{\chi},
\end{aligned}
$$

com $\delta_{\chi}=4 \chi_{0} / \Delta_{+}$sendo o shift de frequência relativo à presença do coeficiente de squeezing. Como observado na análise em [49], a modulação da frequência acopla somente estados $\left|\varphi_{m, \mathcal{D}}\right\rangle \leftrightarrow\left|\varphi_{m \pm 2, \mathcal{D}}\right\rangle$ ou $\left|\varphi_{m>0,-\mathcal{D}}\right\rangle \leftrightarrow\left|\varphi_{m \pm 2,-\mathcal{D}}\right\rangle$. O primeiro caso ocorre quando o átomo está predominantemente no estado fundamental, enquanto o segundo ocorre predominantemente no estado excitado do átomo. 
O foco aqui será a modulação $\eta^{(d)}=2 \omega_{g}$, que produz o ECDg e consiste na criação ou aniquilação de pares de fótons a partir de $m$, sempre partindo do estado atômico fundamental representado pela aproximação $\left|\varphi_{m, \mathcal{D}}\right\rangle \simeq|g, m\rangle$ (expansão em ordem zero dos EV no RD). Tal modulação fornece o Hamiltoniano efetivo

$$
\begin{aligned}
\tilde{H}_{e f f} & \left.=\sum_{m=0}^{\infty} \theta_{m} e^{-i t\left(\lambda_{m+2, \mathcal{S}}-\lambda_{m, \mathcal{T}}-\eta^{(d)}\right.}\right)\left|\varphi_{m, \mathcal{T}}\right\rangle\left\langle\varphi_{m+2, \mathcal{T}}\right|+\text { h.c. } \\
& \simeq \sum_{m=0}^{L_{m a ́ x}}\left(\theta\left|\varphi_{m, \mathcal{D}}\right\rangle\left\langle\varphi_{m+2, \mathcal{D}}\left|+\theta^{*}\right| \varphi_{m+2, \mathcal{D}}\right\rangle\left\langle\varphi_{m, \mathcal{D}}\right|\right)
\end{aligned}
$$

onde $L_{\text {máx }}$ representa o número máximo de excitações permitidas. Tal tratamento aproximado permite levar em conta os efeitos de saturação de uma forma simplificada [43,49]. Os coeficientes obtidos através de (2.13) são

$$
\begin{aligned}
\theta_{m} & =\frac{i \delta_{-} \Omega_{0}}{2 \Delta_{+}} \sqrt{(m+1)(m+2)} \Upsilon^{(d)}, \\
\Upsilon^{(d)} & =\left(\frac{\varepsilon_{\omega}^{(d)}}{\omega_{0}}+\frac{\varepsilon_{\Omega}^{(d)}}{\omega_{0}}-2 \frac{\varepsilon_{g}^{(d)}}{g_{0}}+i \frac{\Delta_{+} \varepsilon_{\chi}^{(d)}}{\delta_{-} \Omega_{0}}\right) .
\end{aligned}
$$

A dinâmica unitária desse Hamiltoniano foi estudada em [43], onde foi mostrado que geração de fótons do vácuo sofre satuaração devido ao efeito Kerr. Ainda, a DU mostra que somente estados $\tilde{\rho}_{m, m}^{\mathcal{D}, \mathcal{D}}$ serão populados

$$
\begin{gathered}
\frac{d}{d t} \tilde{\rho}_{0,0}^{\mathcal{D}, \mathcal{D}}=-2\left(\theta^{*} \tilde{\rho}_{0,2}^{\mathcal{D}, \mathcal{D}}\right)^{i} \\
\frac{d}{d t} \tilde{\rho}_{0,2}^{\mathcal{D}, \mathcal{D}}=-i\left[\theta \tilde{\rho}_{2,2}^{\mathcal{D}, \mathcal{D}}-\theta \tilde{\rho}_{0,0}^{\mathcal{D}, \mathcal{D}}-\theta^{*} \tilde{\rho}_{0,4}^{\mathcal{D}, \mathcal{D}}\right] \\
\frac{d}{d t} \tilde{\rho}_{0, M>2}^{\mathcal{D}, \mathcal{D}}=-i\left[\theta \tilde{\rho}_{2, M}^{\mathcal{D}, \mathcal{D}}-\delta_{M-2 \leq L} \theta \tilde{\rho}_{0, M-2}^{\mathcal{D}, \mathcal{D}}-\delta_{M \leq L} \theta^{*} \tilde{\rho}_{0, M+2}^{\mathcal{D}, \mathcal{D}}\right] \\
\frac{d}{d t} \tilde{\rho}_{N>0, M>0}^{\mathcal{D}, \mathcal{D}}=-i\left[\left(\delta_{N \leq L} \theta \tilde{\rho}_{N+2, M}^{\mathcal{D}, \mathcal{D}}+\theta^{*} \delta_{N-2 \leq L} \tilde{\rho}_{N-2, M}^{\mathcal{D}, \mathcal{D}}\right)-\left(\delta_{M-2 \leq L} \theta \tilde{\rho}_{N, M-2}^{\mathcal{D}, \mathcal{D}}+\delta_{M \leq L} \theta^{*} \tilde{\rho}_{N, M+2}^{\mathcal{D}, \mathcal{D}}\right)\right] .
\end{gathered}
$$

Através das equações fornecidas pela DU, vemos que $\tilde{\rho}_{m, m}^{-\mathcal{D},-\mathcal{D}}(0)=0$. Definindo o conjunto $\{0, \mathcal{D}\}$ correspondendo formalmente a $\{0,-\}$, a RAP terá como equações

$$
\begin{gathered}
\frac{d}{d t} \tilde{\rho}_{0,0}^{-,-}=\gamma \frac{g_{0}^{2}}{\Delta_{-}^{2}} \tilde{\rho}_{1,1}^{\mathcal{D} \cdot \mathcal{D}}, \\
\frac{d}{d t} \tilde{\rho}_{N>0, N}^{\mathcal{D}, \mathcal{D}}=\gamma\left(\frac{g_{0}^{2}}{\Delta_{-}^{2}}(N+1) \tilde{\rho}_{N+1, N+1}^{\mathcal{D}, \mathcal{D}}-\frac{g_{0}^{2}}{\Delta_{-}^{2}} N \tilde{\rho}_{N, N}^{\mathcal{D}, \mathcal{D}}\right),
\end{gathered}
$$




$$
\begin{aligned}
\frac{d}{d t} \tilde{\rho}_{N>0, M>0, M \neq N}^{\mathcal{D}, \mathcal{D}} & =-\frac{\gamma}{2} \frac{g_{0}^{2}}{\Delta_{-}^{2}}(N+M) \tilde{\rho}_{N M}^{\mathcal{D}, \mathcal{D}}, \\
\frac{d}{d t} \tilde{\rho}_{0, M \neq N}^{\mathcal{D}, \mathcal{D}} & =-\frac{\gamma}{2} \frac{g_{0}^{2}}{\Delta_{-}^{2}} M \tilde{\rho}_{0 M}^{\mathcal{D}, \mathcal{D}},
\end{aligned}
$$

ao passo que a RPC apenas permite que tais equações sejam definidas

$$
\begin{gathered}
\frac{d}{d t} \tilde{\rho}_{0,0}^{-,-}=\kappa\left(1-\frac{g_{0}^{2}}{\Delta_{-}^{2}}\right) \tilde{\rho}_{1,1}^{\mathcal{D}, \mathcal{D}}, \\
\frac{d}{d t} \tilde{\rho}_{N>0, N}^{\mathcal{D}, \mathcal{D}}=\kappa\left(1-\frac{g_{0}^{2}}{\Delta_{-}^{2}}\right)\left((N+1) \tilde{\rho}_{N+1, N+1}^{\mathcal{D}, \mathcal{D}}-N \tilde{\rho}_{N, N}^{\mathcal{D}, \mathcal{D}}\right), \\
\frac{d}{d t} \tilde{\rho}_{0, M>0}^{\mathcal{D}, \mathcal{D}}=-\frac{\kappa\left(1-\frac{g_{0}^{2}}{\Delta_{-}^{2}}\right)}{2} M \tilde{\rho}_{0, M}^{\mathcal{D}, \mathcal{D}}, \\
\frac{d}{d t} \tilde{\rho}_{N>0, M>0, M \neq N}^{\mathcal{D}, \mathcal{D}}=-\frac{\kappa\left(1-\frac{g_{0}^{2}}{\Delta_{-}^{2}}\right)}{2}(N+M) \tilde{\rho}_{N, M}^{\mathcal{D}, \mathcal{D}} .
\end{gathered}
$$

Observa-se que a relaxação atômica pura e a relaxação pura da cavidade são qualitativamente semelhantes considerando que

$$
\gamma \frac{g_{0}^{2}}{\Delta_{-}^{2}}=\kappa\left(1-\frac{g_{0}^{2}}{\Delta_{0}^{2}}\right)
$$

ou seja, é como se a taxa de relaxação da cavidade sofresse um aumento dado por $\kappa=\gamma \frac{g_{0}^{2}}{\Delta_{-}^{2}}$. 


\section{Capítulo 6}

\section{Análise Numérica dos Efeitos de Dissipação em EDQ de circuitos}

Até o momento os efeitos dissipativos de circuitos quânticos supercondutores em que os parâmetros do qubit sofrem modulação temporal externa foram analisados apenas assintóticamente. Como mostrado, a dinâmica unitária para todos os regimes é alterada. Entretanto, o preço pago na análise anterior foi não ter informação a respeito do comportamento do sistema ao longo do tempo. Durante a construção da EMEV foi levado em conta o acoplamento entre os subsistemas, tendo o papel de protagonista. Como a EMEV propõe uma descrição mais completa para o fenômeno de dissipação em EDQ de circuitos, a proposta deste capítulo é analisar numericamente como o sistema se comporta sob tal descrição e, em seguida, verificar se há diferenças quando comparada à EMP. Além da comparação númerica exata entre os dois modelos de dissipação, serão analisadas as soluções aproximadas obtidas no capítulo anterior. A abordagem para resolver a EM será o método de integração de Runge-Kutta em quinta e sexta ordem, de modo que as equações diferenciais para os elementos da matriz densidade serão o alvo. Além disso, a análise não contemplará o chamado regime de acomplamento ultra-forte $\left(g_{0} \lesssim \omega_{0}\right)[2,3,16,45]$ limitando a análise ao regime de acomplamento fraco estudado em [44] e o acoplamento forte. O acoplamento fraco é definido quando a constante de acoplamento átomo-campo $g_{0}$ é muito pequena comparada à frequência da cavidade $\omega_{0}, g_{0} \ll \omega_{0}$.

Quando a comparação entre $g_{0}$ e $\omega_{0}$ fornece a relação $g_{0} \lesssim 10^{-1} \omega_{0}$ o regime de acoplamento será definido como o acoplamento forte [2].

Através da solução númerica é possível saber como as populações se modificam ao longo do 
tempo, o que permite o cálculo do número médio de excitações do qubit e da cavidade e como a distribuição de fótons se altera através do parâmetro $Q$ de Mandel [17]. O parâmetro $Q$ de Mandel será uma maneira conveniente de caracterizar se os estados serão não-clássicos através da distribuição da probabilidade de fótons

$$
Q=\frac{\left\langle\left(\Delta \hat{n}^{2}\right)\right\rangle-\langle\hat{n}\rangle}{\langle\hat{n}\rangle}=\frac{\left\langle\hat{n}^{2}\right\rangle-\langle\hat{n}\rangle^{2}-\langle\hat{n}\rangle}{\langle\hat{n}\rangle} .
$$

Valores positivos de $Q$ indicam uma estatística super-poissoniana, por exemplo, estado térmico ou vácuo comprimido. Se $Q$ for nulo, a distribuição será poissoniana e podemos citar como exemplo o estado coerente. Se $Q$ for negativo sua distribuição será sub-poissoniana, que fornece um estado puramente quântico, como o estado estado de Fock (ou superposição deles) ou estado coerente comprimido.

A análise númerica para o comportamento do fenômeno de criação e aniquilação de estados emaranhados com duas excitações a partir do EZE foi feita com os parâmetros $g_{0}=10^{-1} \omega_{0}, \Delta_{-}=0$, $\varepsilon_{\Omega}=10^{-1} \Omega_{0}$ e $\eta^{(r)}=2 \omega_{0}+g_{0} \sqrt{2}$, obtendo $|\theta| \approx 2 \times 10^{-3} g_{0}$. O valor usual para a frequência da cavidade é $\omega_{0} / 2 \pi=8 \mathrm{GHz}$ [44], entretanto, para fins da análise, apenas as razões entre parâmetros foram consideradas, tendo sido utilizado $\omega_{0}=1$. A taxa de dissipação utilizada para RAP foi $\gamma=5 \times 10^{-4} \omega_{0}$. O número médio de fótons criados sob relaxação atômica pura foi obtido a partir da solução geral das equações analíticas aproximadas, representado na figura 6.1-(c). Além disso, a solução exata para o problema sob relaxação atômica, exibida na figura 6.1-(b), mostra que o valor esperado é aproximadamente igual à solução analítica para o número médio de fótons. Todavia, a escala de tempo adimensional $\tau^{\prime}$ utilizada nas figuras 6.1-(a),(c),(e) depende do parâmetro $|\theta|$ $\left(\tau^{\prime}=t \times|\theta|\right)$, ao passo que a solução exata, mostrada nas figuras 6.1- $(b),(d),(f)$, foi obtida utilizandose a escala de tempo adimensional $\tau=t \times g_{0}$. O resultado teórico previa $\langle\hat{n}\rangle=1,5$ na ausência de dissipação, entretanto sob relaxação atômica pura esse valor esperado é reduzido. Como mostrado na figura (6.1) ambas as equações mestras mostram resultados semelhantes para a taxa de dissipação utilizada. Além disso, como mostrado em [44] ambas predizem resultados semelhantes no regime de acoplamento fraco $g \ll \omega_{0}$.

Além do exposto anteriormente, o fator $Q$ mostra que sob o efeito desse canal de dissipação a distribuição tende a ser sub-poissoniana. Ainda, como pode ser observado, tanto na solução analítica aproximada (figura 6.1-(e)) quanto na solução exata para as duas equações mestras (figura 6.1-(d)), 
Figura 6.1: Geração de excitações a partir do EZE no regime ressonante. (a) Comportamento analítico aproximado para as populações. (b) Número médio de fótons criados sob RAP para a EMP (EMEV) em preto (vermelho). (c) Número médio de fótons obtidos a partir da solução analítica aproximada. (d) Probabilidade de excitação atômica para EMP (EMEV) em preto (vermelho). (e) Probabilidade de excitação atômica para solução analítica aproximada. (f) fator Q de Mandel.
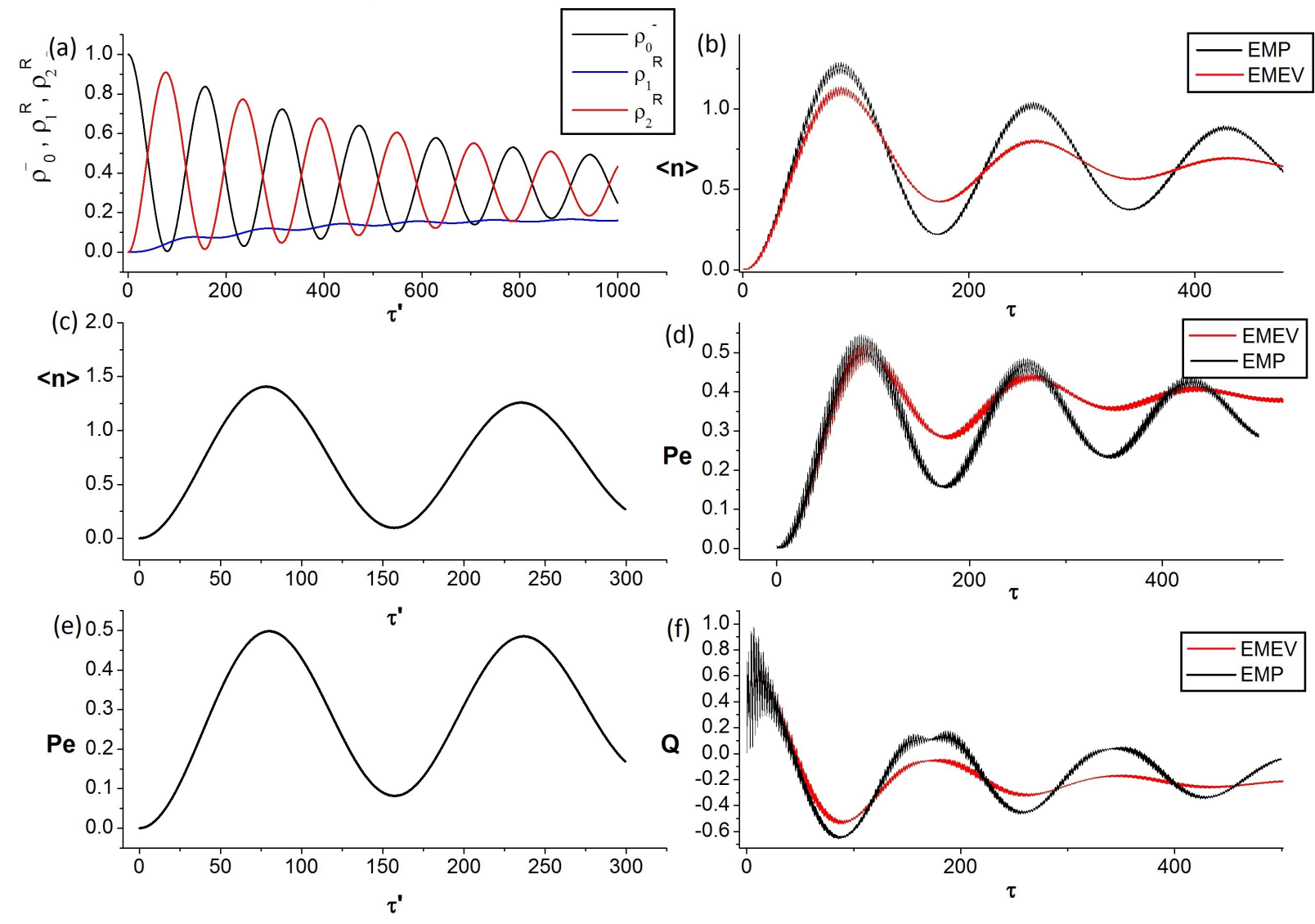
o sistema tem probabilidade de excitação atômica não-nula. Para tempos maiores de observação, $P_{e}$ se estabiliza em valores não-nulos. Na figura 6.1-(a), o comportamento das populações pode ser observado e, para tempos maiores de observação, tendem aos valores previstos analiticamente. As populações $\tilde{\rho}_{0,0}^{-,-}, \tilde{\rho}_{1,1}^{\mathcal{R}, \mathcal{R}}$ e $\tilde{\rho}_{2,2}^{\mathcal{R}, \mathcal{R}}$ foram representadas por $\tilde{\rho}_{0}^{-}, \tilde{\rho}_{1}^{R}$ e $\tilde{\rho}_{2}^{R}$, respectivamente.

Figura 6.2: Fenômeno ECDg.(a) Número médio de fótons sem dissipação. (b) Comparativo númerico exato para o número médio de fótons sob relaxação atômica pura (preto) e relaxação pura da cavidade (vermelho). (c) Probabilidade de excitação atômica para RAP (RPC) em preto (vermelho). (d) Fator Q de Mandel para RAP (RPC) em preto(vermelho).
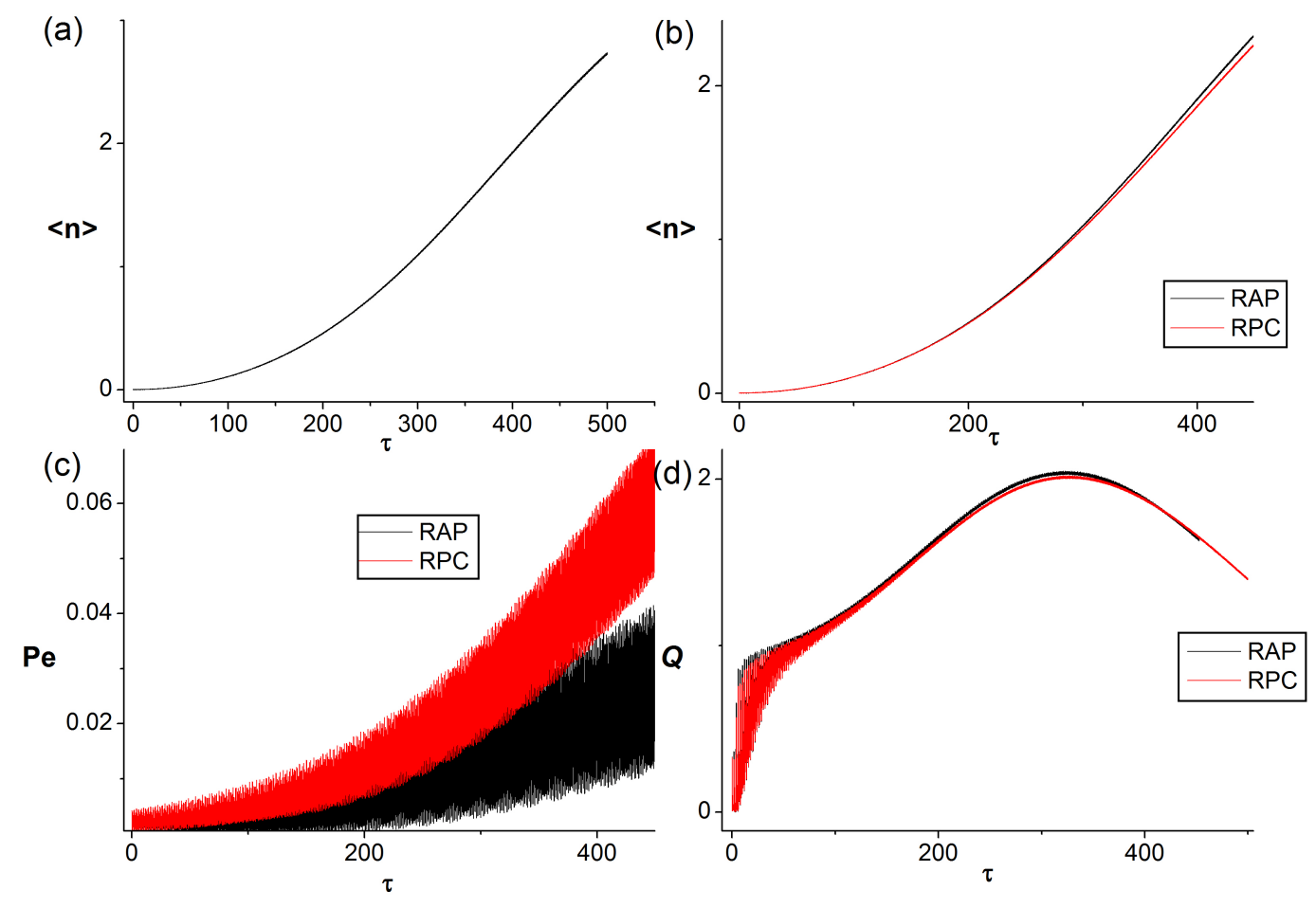

No ECDg, foi demonstrado analíticamente que as taxas de relaxação do átomo e do campo levam a comportamento semelhante, pois o decaimento atômico permite que a cavidade dissipe energia com taxa efetiva $\kappa=\gamma \frac{g_{0}^{2}}{\Delta_{0}^{2}}$. Os parâmetros $g_{0}=10^{-1} \omega_{0}, \Delta_{-}=10 g_{0}, \varepsilon_{\Omega}=10^{-1} \Omega_{0} \mathrm{e}$ $\eta^{(d)}=2\left(\omega_{0}+\delta_{-}-\delta_{+}\right)$foram utilizados na análise númerica, com tempo adimensional $\tau=t \times g_{0}$. Como previsto anteriormente, a figura 6.2-(b) mostra que para tempos curtos o número médio de fótons criados sob RAP e RPC, utilizando a EMP com taxas $\gamma=\kappa=2,5 \times 10^{-4} \omega_{0}$, respectivamente, 
é aproximadamente igual para ambos os casos. Além do mais, como mostrado em [44], ocorre saturação no número médio de fótons criados para valores não-nulos de $\left(\lambda_{m+2, \mathcal{D}}-\lambda_{m, \mathcal{D}}-\eta^{(d)}\right) \propto m$ (para valores grandes de $m$ ) sem que seja relacionado aos efeitos dissipativos. O fenômeno de colapsoressurgimento de $\langle\hat{n}\rangle$ [9], [34] é observado em [44], mas sem que seja associado à emissão ou reabsorção de fótons pelo átomo. A probabilidade de excitação do átomo mostrada na figura 6.2-(c) mostra pouca diferença para ambos os canais dissipativos, sendo que sob RAP $P_{e}$ é menor, como esperado intuitivamente. O parâmetro $Q$ indica uma distribuição super-poissoniana para todos os tempos, sem que haja distinção entre as relaxações. Isso indica que para tempos curtos o estado criado é aproximadamente o vácuo comprimido. A diminuição de $Q$ para tempos maiores deve-se aos efeitos de saturação comentados acima. 
Figura 6.3: Fenômeno anti-ECD sob dissipação via RAP. (a) Distribuição das populações ao longo do tempo adimensional $\tau^{\prime}=t \times|\theta|$. (b) Número médio de fótons criados e aniquilados em função do tempo. (c) Probabilidade de excitação atômica. (d) Fator $Q$ ao longo do tempo adimensional $\tau^{\prime}$.
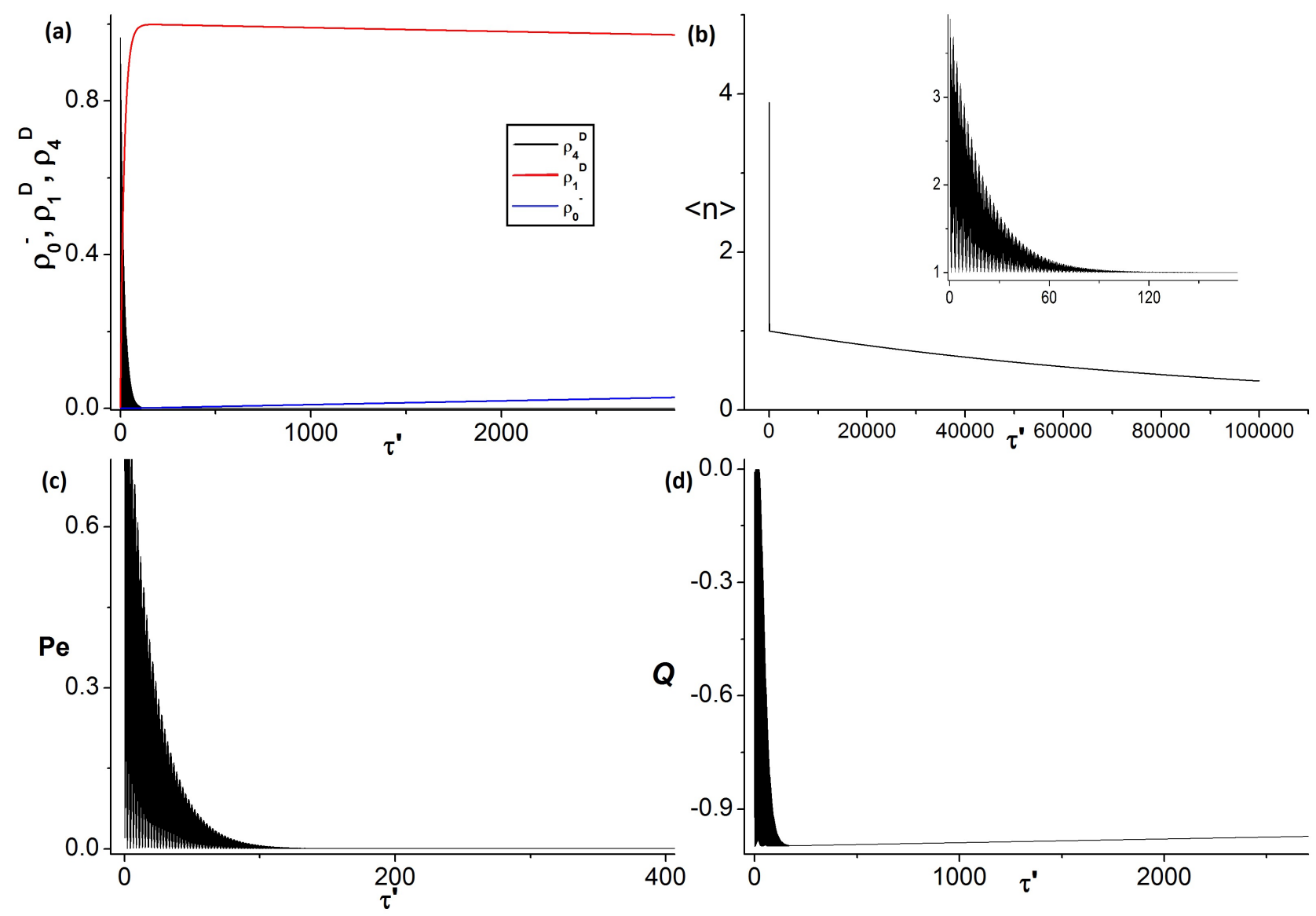

A descrição teórica do regime anti-ECD previa a transição entre os estados na forma $|g, k\rangle \leftrightarrow$ $|e, k-3\rangle$, entretanto, a solução analítica assintótica demonstrou que o sistema vai ao EZE sob RAP como única solução não nula. A figura 6.3 trata de um caso específico para $k=4$ e foi obtida a partir da solução analítica do capítulo anterior, considerando a expansão até segunda ordem em $\frac{g_{0}}{\Delta_{-}}$. Os parâmetros utilizados foram $g_{0}=5 \times 10^{-2} \omega_{0}, \Delta_{-}=10 g_{0}, \varepsilon_{\Omega}=10^{-1} \Omega_{0}, \eta_{4}^{(A)} \approx 5,04$ e $|\theta| \sim 10^{-4} g_{0}$, normalizando os parâmetros em relação a $\omega_{0}=2$. A descrição analítica para a dinâmica unitária prevê que três fótons são eliminados seguido de uma excitação atômica. Analiticamente se espera um comportamento $|g, 4\rangle \rightarrow|g, 0\rangle$ sob RAP, pois foi observado que somente o estado $|g, 0\rangle$ tinha solução 
assintótica não-nula. Na figura 6.3-(a) a população $\rho_{4,4}^{\mathcal{D}, \mathcal{D}}$ (representada por $\rho_{4}^{D}$ ) vai à zero em função do tempo adimensional $\tau^{\prime}$, enquanto outras populações têm probabilidade não-nula por um intervalo de tempo muito curto até ir a zero. Em seguida, a população $\rho_{1,1}^{\mathcal{D}, \mathcal{D}}$ aparece e, à medida que o tempo adimensional passa, a probalidade $\rho_{0,0}^{-,-}$emerge caracterizado por um processo muito lento de emissão espontânea. A figura 6.3-(b) mostra como o número médio de fótons não obedece à evolução unitária e lentamente vai à zero. Nesse mesmo intervalo de tempo, a probabilidade de excitação atômica vai à zero, indicando a transição de $|e, 1\rangle \rightarrow|g, 1\rangle$. À medida que há um decaimento para o EZE, o fator $Q$ mostra que distribuição dos fótons tende a uma distribuição poissoniana (para tempos muito longos $Q$ vai a zero). É importante salientar que no regime AECD a resposta do sistema à perturbação harmônica consiste na tranferência de energia do sistema para o agente externo.

Figura 6.4: Fenômeno anti-ECD: solução exata para EMP. (a) Número médio de fótons na ausência (presença) de dissipação em preto (vermelho). (b) Probabilidade de excitação atômica $P_{e}$ sem (com) a presença de dissipação em preto (vermelho). (c) Fator $Q$ em função do tempo sem (com) a presença de dissipação em preto (vermelho). (d) Probabilidade para o estado atômico fundamental sem (com) efeitos dissipativos em preto (vermelho).

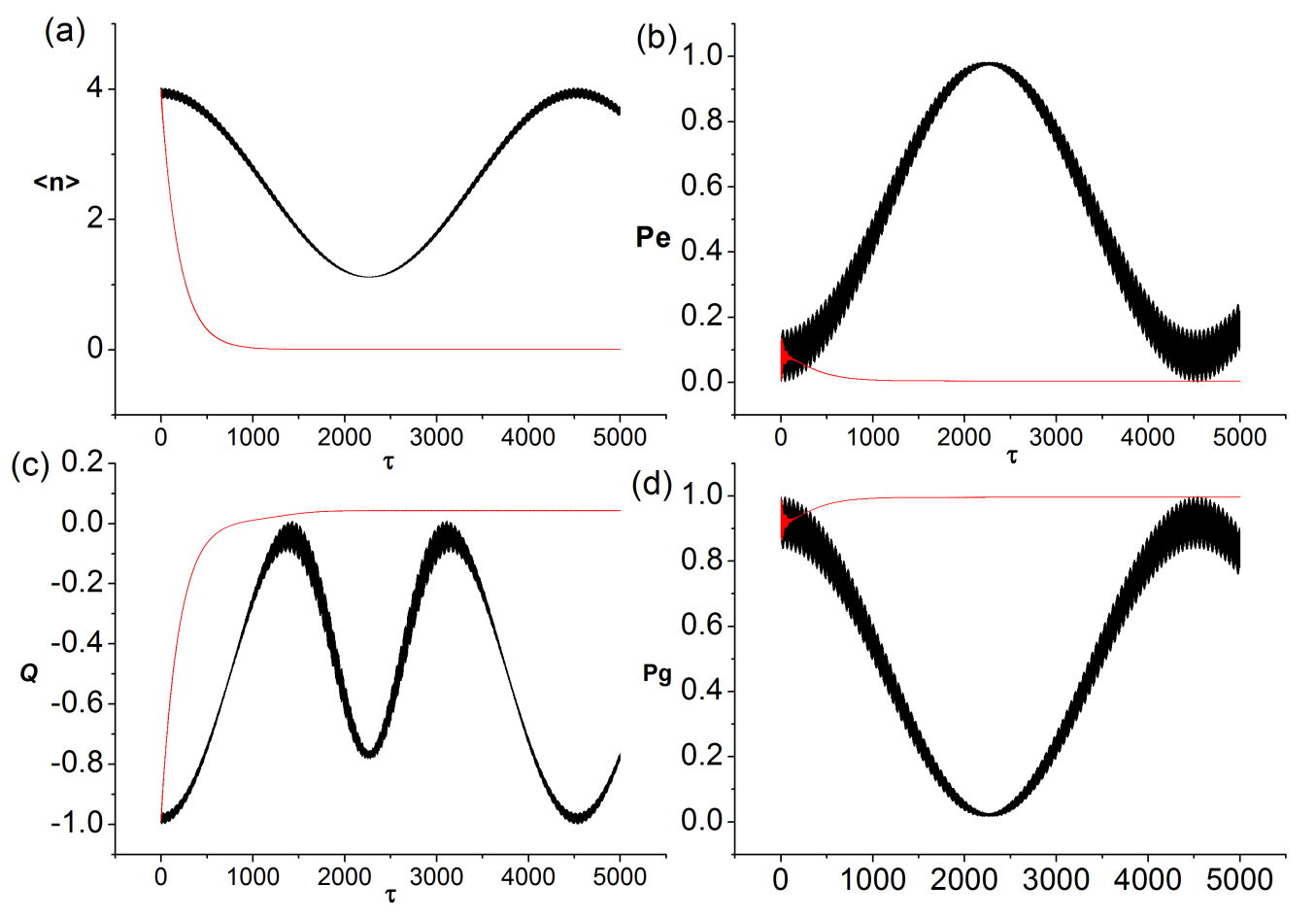


Diferentemente, o processo sofre uma drástica modificação quando os três canais de dissipação estão presentes, como pode ser observado na figura 6.4. As taxas de dissipação utilizadas foram $\gamma=\gamma_{\phi}=\kappa=2,5 \times 10^{-4} \omega_{0}$ e, como é possível observar na figura 6.4-(a), o número médio de fótons criados vai rapidamente a zero. Na figura 6.4, a análise se restringiu a um intervalo de tempo limitado, porém para tempos maiores o número médio de fótons criados repete periodicamente o comportamento mostrado sem a presença de dissipação. A mudança do estado fundamental para o estado excitado do átomo pode ser observada na figura 6.4-(b) na linha preta, entretanto, a presença dos três canais dissipativos alteram drasticamente o comportamento com essa probabilidade indo a zero. Além disso, a estatística tende a ser poissoniana à medida que o número médio de fótons vai a zero, como visto na figura 6.4-(c). O efeito é, então, considerado um efeito fraco, necessitando que modulação tenha um ajuste muito fino e a perturbação mantida por tempos prolongados. O resultado previsto e mostrado em [44] prediz que ambas as equações mestras produzem praticamente o mesmo resultado quando a análise é feita no regime de acoplamento fraco. 
Figura 6.5: Dupla modulação da frequência no regime ressonante. (a) Número médio de fótons sob dissipação para a EMP (EMEV) em preto (vermelho).(b) Fator Q para EMP (EMEV) em preto (vermelho). (c) Probabilidade de excitação atômica $P_{e}$ descrita pela EMP (EMEV) em preto (vermelho).

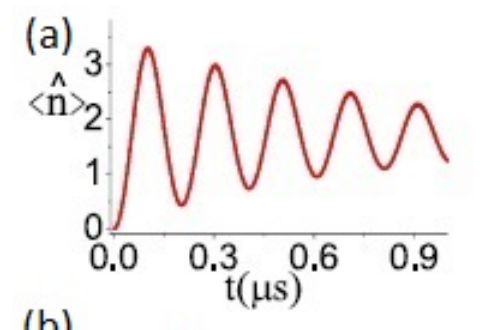

(b)
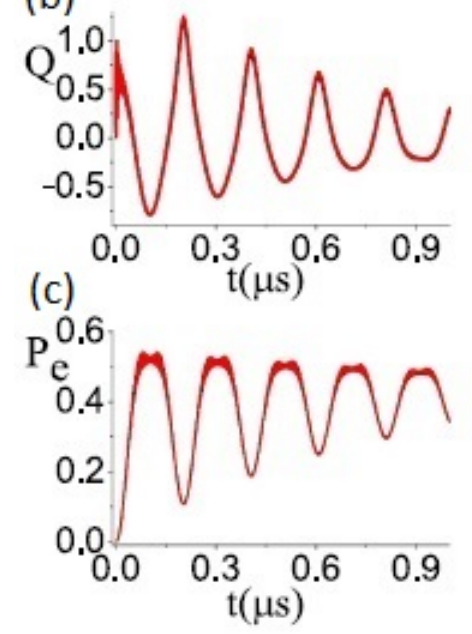

Quando o sistema foi modulado em multi-frequências, mais especificamente em modulação de duas frequências, os efeitos foram incrementados. Com a modulação simultânea aplicada a $\Omega$ e $g$ no regime ressonante, com frequências $\eta^{(r)}=2 \omega_{0}+g_{0} \sqrt{2}$ e $\eta^{(r 2)}=2 \omega_{0}+g_{0}(2-\sqrt{2})$, respectivamente, combina-se simultaneamente o acoplamento $\left|\varphi_{0,-}\right\rangle \rightarrow\left|\varphi_{2,+}\right\rangle$ com acoplamento $\left|\varphi_{2,+}\right\rangle \rightarrow\left|\varphi_{4,+}\right\rangle$. Tal modulação foi realizada e mostrada em [44], com os parâmetros $g_{0}=5 \times 10^{-2} \omega_{0}, \Delta_{-}=0, \varepsilon_{\Omega}=$ $5 \times 10^{-2} \Omega_{0}, \varepsilon_{g}=1,97 \times 10^{-2} g_{0}$ e com taxas de dissipação $\gamma=\gamma_{\phi}=\kappa=5 \times 10^{-4} \omega_{0}$, onde foram obtidos os resultados da figura 6.5. É possível obter o coeficiente $\left|\theta_{2}\right|$ a partir de $|\theta|$ conhecendo-se o estado inicial do sistema como mostrado no estudo analítico do capítulo anterior. No caso em questão, as estimativas forceneram $|\theta|,\left|\theta_{2}\right| \sim 10^{-3} g_{0}$. Entretanto, é importante salientar que tal resultado foi obtido sob o regime de acoplamento fraco. Ademais, o efeito de dissipação descrito pelas duas equações mestras é indistinguível para taxas de dissipação moderadas e a geração de 
fótons incrementada devida à multi-modulação pode ser observada na figura 6.5-(a). Além do mais, a distribuição de fótons dado pelo fator $Q$ permanece semelhante ao longo do tempo para as duas equações e confirma a geração de estados não-clássicos.

O regime anti-Jaynes-Cummings foi estudado analiticamente prevendo seu comportamento alterado devido à dissipação. Todavia, carece, ainda, de ser estudado através da solução numérica exata. No capítulo anterior previu-se sob algumas condições que a dinâmica dissipativa sob RAP fornecia a probabilidade $\tilde{\rho}_{1,1}^{\mathcal{D}, \mathcal{D}} \approx 1\left(\tilde{\rho}_{1}^{\mathcal{D}}\right)$. A figura 6.6-(a) mostra como essa população se comporta em função do tempo à medida que $\tilde{\rho}_{0,0}^{-,-}\left(\tilde{\rho}_{0}^{-}\right)$se aproxima de zero. A figura 6.6-(c) mostra como as populações se comportam quando o sistema sofre com o processo de dissipação somente via RCP, mostrando que $\tilde{\rho}_{1,1}^{-\mathcal{D},-\mathcal{D}}\left(\tilde{\rho}_{1}^{-\mathcal{D}}\right)$ tende aproximadamente a 1 , como previsto no comportamento analítico assintótico. Os parâmetros usados na modulação foram $g_{0}=5 \times 10^{-2} \omega_{0}, \Delta_{-}=10 g_{0}, \varepsilon_{\Omega}=10^{-1} \Omega_{0}$, com taxas $\gamma=\gamma_{\phi}=\kappa=2,5 \times 10^{-4} \omega_{0}$. Nesse caso, $|\theta| \approx 8 \times 10^{-3} g_{0}$ e o tempo adimensional considerado foi $\tau^{\prime}=t \times|\theta|$. 
Figura 6.6: Regime anti-Jaynes-Cummings. (a) Comportamento das populações em função do tempo adimensional $\tau^{\prime}=t \times|\theta|$ sob dissipação gerada por RAP. (b) Número médio de fótons criados sem dissipação em preto e com dissipação analisada para EMP (EMEV) em azul (vermelho). (c) Comportamento das populações em função do tempo adimensional $\tau^{\prime}$ sob dissipação gerada por RCP. (d) Probabilidade de excitação atômica sem dissipação em preto e com dissipação analisada para EMP (EMEV) em azul (vermelho). (e) Número médio de fótons gerados sob a presença de dissipação analisada para RAP (RPC) em preto (vermelho). (f) Fator $Q$ sem dissipação na linha preta e com dissipação analisada para EMP (EMEV) em azul (vermelho).
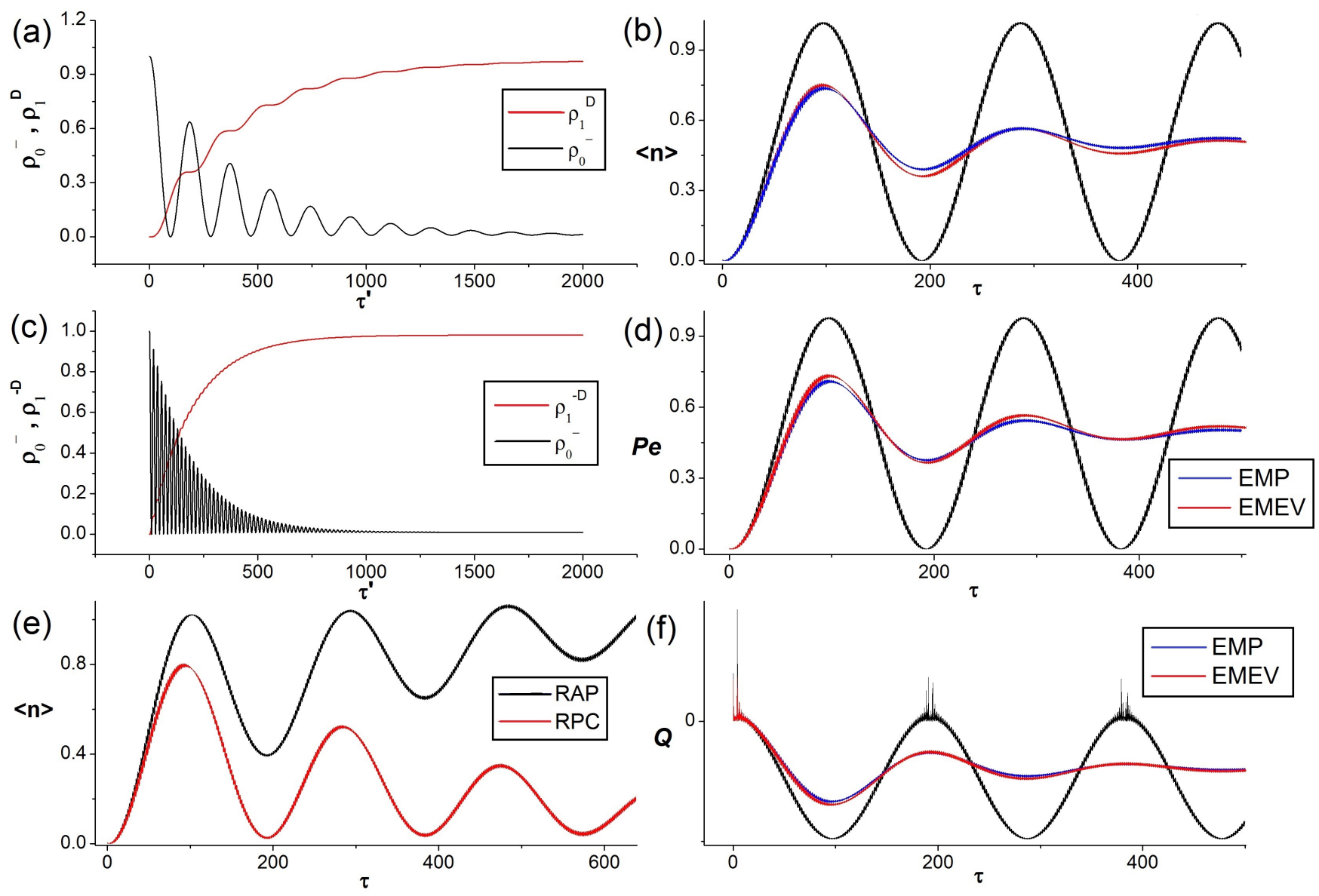

O regime AJC é o efeito que descreve o processo de mudança do estado fundamental para o estado atômico excitado simultânea à criação de um fóton [49], [50]. A figura 6.6-(b) demonstra como o número médio de fótons se comporta em função do tempo adimensional $\tau=t \times g_{0}$, tornando 
possível a comparação entre os dois modelos de dissipação utilizados. Como é possível perceber, a EMP e a EMEV não demonstram distinção e a geração de fótons ainda é possível com a presença dos três canais de dissipação. A figura 6.6-(d) mostra que a probabilidade de excitação atômica quando há dissipação é não-nula e sem distinção entre as equações mestras utilizadas. Quanto ao fator $Q$ pode-se ver na figura 6.6-(f) que o caráter não-clássico prevalece durante a dinâmica do sistema e para tempos mais longos a distribuição permanece sub-poissoniana. Já os ruídos presentes em $Q$ são oriundos de dispersões de fótons que são amplificadas quando $\langle\hat{n}\rangle \approx 0$. O número médio de fótons criados quando há dissipação via relaxação atômica pura ou via relaxação pura da cavidade é visto na figura 6.6-(e) descritos pela EMP. 
Figura 6.7: Dupla modulação da frequência: regime AECD incrementado. (a) Número médio de fótons sem (com) a presença de dissipação em preto (vermelho). (b) Probabilidade de excitação atômica sem (com) a presença de dissipação em preto (vermelho). (c) Fator Q sem (com) a presença de dissipação em preto (vermelho). (d) Probabilidade para o estado atômico fundamental sem (com) a presença de dissipação em preto (vermelho).
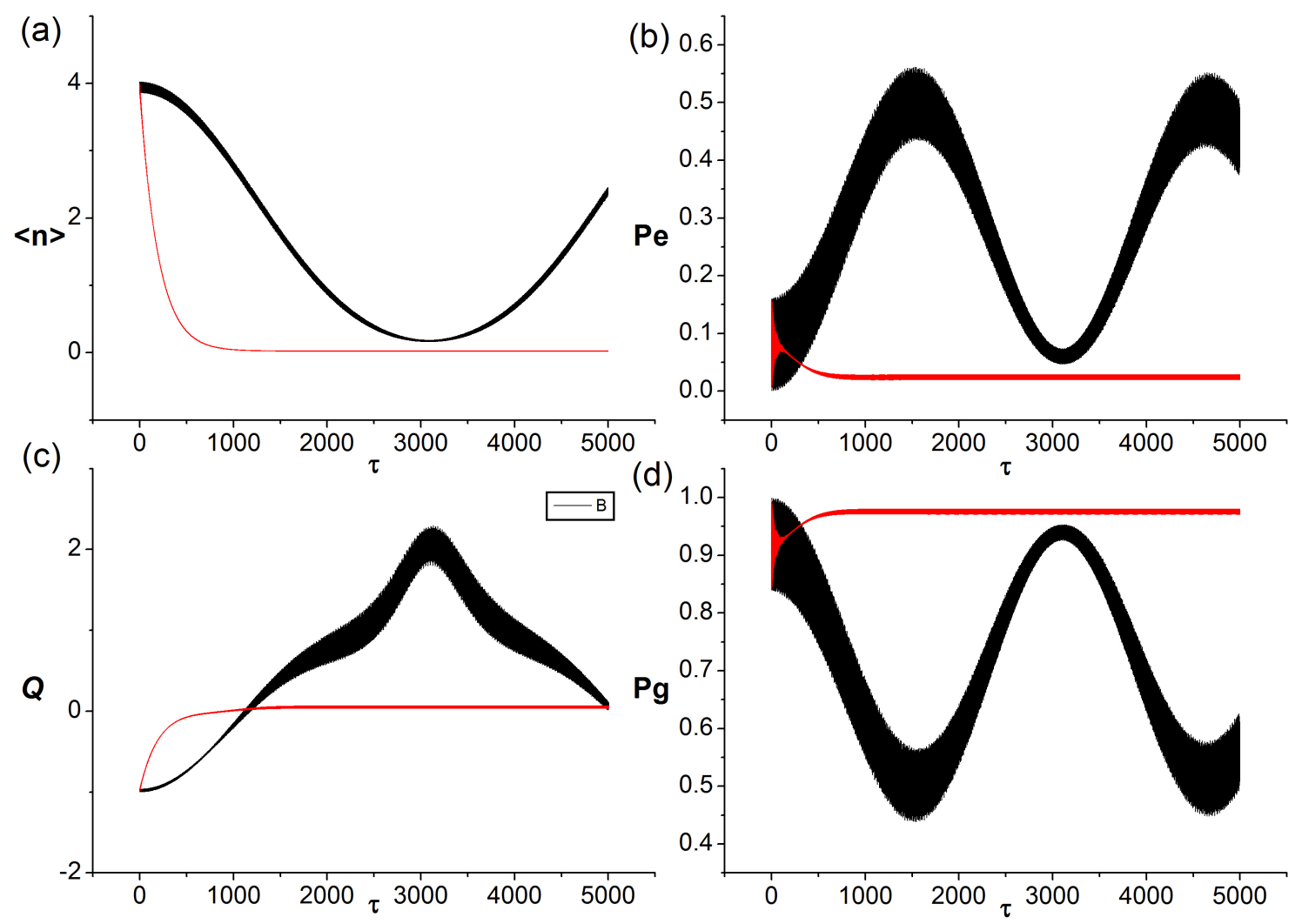

A incrementação do regime anti-ECD pôde ser analisada no capítulo anterior. Como visto, com a dupla modulação da frequência quatro fótons eram subtraídos do estado $|g, k\rangle\langle g, k|$. A modulação de $g$ com frequência $\eta_{4}^{(A 2)} \simeq 2.98$ (considerando $\omega_{0}=2$ ), com profundidade da modulação $\varepsilon_{g} \approx 1,47 \times 10^{-3} g_{0}$, será utilizada e os demais parâmetros são os mesmos utilizados para o regime AECD. Nesse caso, com taxas de dissipação $\gamma=\gamma_{\phi}=\kappa=2,5 \times 10^{-4} \omega_{0}$ o comportamento do sistema é completamente alterado. Na figura 6.7-(a) o número médio de fótons pode ser observado sem a presença de dissipação na curva em linha preta e quando sob dissipação observa-se $\langle\hat{n}\rangle$ indo imediatamente a zero na curva em vermelho. Para a análise dos efeitos dissipativos a EMP foi utilizada. 
Semelhante à análise do regime AECD, a presença dos três canais dissipativos destroem o interessante comportamento da incrementação do regime AECD. A probabilidade de excitação atômica vai rapidamente a zero nesse caso e a probabilidade para o estado atômico fundamental é máxima quando a dissipação está presente.

Figura 6.8: Dupla modulação da frequência: regime AECD incrementado. Taxas de dissipação moderadas para EMP. (a) Número médio de fótons sem (com) a presença de dissipação em preto (vermelho). (b) Probabilidade de excitação atômica sem (com) a presença de dissipação em preto (vermelho). (c) Fator Q sem (com) a presença de dissipação em preto (vermelho). (d) Probabilidade para o estado atômico fundamental sem (com) a presença de dissipação em preto (vermelho).
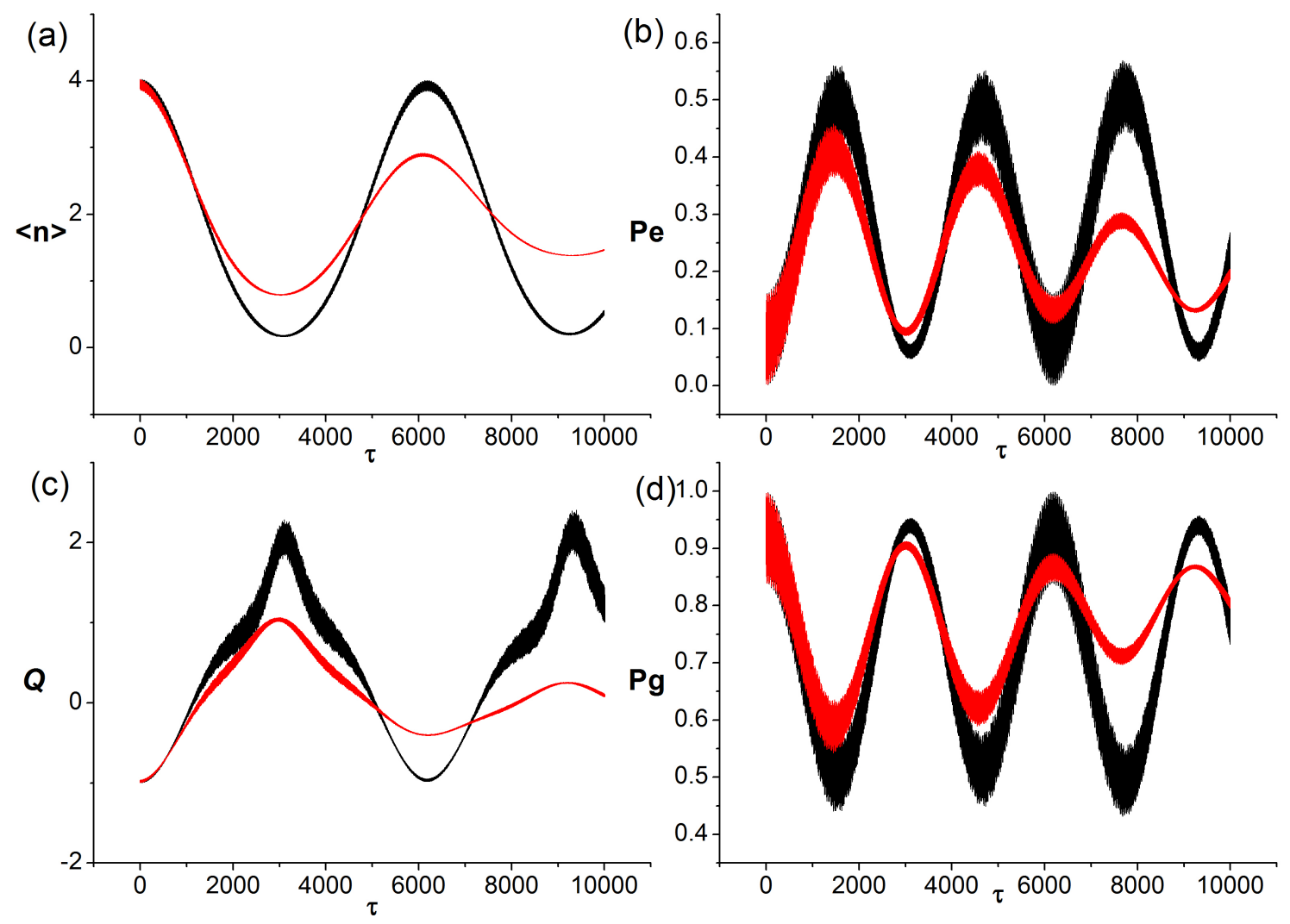

Assim como no regime AECD, é necessário um ajuste mais fino dos parâmetros e as taxas de dissipação devem ser mais moderadas para que seja possível a observação do fenômeno [44]. A figura 6.8 mostra que as taxas de dissipação $\gamma=\gamma_{\phi}=\kappa=2,5 \times 10^{-5} \omega_{0}$ possibilitam a observação de excitações sendo destruídas e geradas. Além disso, é necessário que a observação seja para largas 
escalas de tempo. Na figura 6.8-(a) pode-se ver que fótons continuam sendo destruídos e gerados, entretanto há uma redução no contraste em comparação com a dinâmica unitária. Se o fenômeno é observado em tempos maiores, será possível observar que o envelope de $\langle\hat{n}\rangle$ decai exponencialmente, apresentando oscilações periódicas. O regime anti-ECD incrementado, assim como o regime AECD, encontra-se no limiar de implementação experimental devido à necessidade de manter duas modulações muito bem ajustadas. 


\section{Capítulo 7}

\section{Conclusões e Perspectivas}

No presente trabalho, o estudo foi desenvolvido em torno do regime não-estacionário de circuitos de eletrodinâmica quântica. Toda formulação matemática da dinâmica unitária já havia sido previamente estudada em alguns trabalhos a partir da interação de um qubit, nomenclatura utilizada para referir-se a um átomo artificial, com um campo eletromagnético confinado em um ressonador guiade-onda. Para isso, considerou-se a modulação externa de parâmetros do sistema $X=\{\omega, \Omega, g, \chi\}$ de forma periódica ou com certos perfis não-periódicos (superposição de pertubações harmônicas). Uma equação diferencial foi obtida [ver equação (2.12)], através de muitas aproximações, que tornou possível estipular as frequências ressonantes a partir dos autovalores corrigidos obtidos no formalismo dos Estados Vestidos.

Um Hamiltoniano efetivo foi deduzido aqui para que a notação fosse compactada e que, apenas com a frequência ressonante, fornecesse o acoplamento entre os estados quânticos. Assim, os seguintes efeitos foram analisados: geração de fótons do vácuo no regime ressonante; efeito antiJaynes-Cummings; efeito Casimir dinâmico e similares no regime dispersivo. O nosso intuito foi estimar taxas de criação e aniquilação de excitações em circuitos dissipativos de eletrodinâmica quântica. Para isso, duas equações mestras Markovianas foram utilizadas, Equação Mestra Padrão e Equação Mestra na representação dos Estados Vestidos, de forma que se pudesse analisar a dinâmica em circuntâncias reais para diferentes fenômenos. Bastante conhecida na literatura, a primeira equação mestra é de mais fácil manuseio, já que ela opera ou apenas para átomo ou para campo. Devido à sofisticação usada na formulação da segunda equação mestra, que é mais completa, seu manuseio e operação analítica são mais complexos, exigindo que seja resolvida numericamente. 
Inicialmente, uma solução analítica foi obtida para descobrir o comportamento assintótico do sistema (impondo a taxa de interação coerente maior que as taxas de dissipação) e obter uma ideia geral a respeito dos efeitos de diferentes canais dissipativos. Em seguida, a solução numérica computacional foi implementada para que se comparasse as diferentes propostas de dissipação. Os resultados indicam que os fenômenos podem ser implementados realisticamente. Para a maioria dos efeitos, que são de geração de um ou dois pares de excitações a partir do vácuo no regime ressonante, ou geração de um par de excitações no regime dispersivo (AJC), a implementação é mais fácil. Nesses efeitos, a taxa de geração é da ordem de $|\theta| \sim 10^{-3} g_{0}$ enquanto as taxas de dissipação são menores. Já para efeitos de criação de fótons a partir do vácuo via ECD ou aniquilação de duas ou quatro excitações via anti-ECD e anti-ECD incrementado, respectivamente, as taxas são da ordem de $|\theta| \sim 10^{-4} g_{0}$. Os processos de aniquilação de fótons (usando simples- ou duplamodulação) caracterizam-se como processos lentos e necessitam que a dissipação seja mais moderada. Arquiteturas de EDQ de circuitos de ponta podem permitir que essa implementação seja realizada.

Os fenômenos analisados no regime de acoplamento fraco ou forte [2] não apresentaram diferenças significativas na comparação entre as duas equações mestras utilizadas. Análises futuras podem verificar o surgimento de diferenças qualitativas nos resultados para o regime de acoplamento ultra-forte [16]. Os requisitos para implementar em laboratório os fenômenos aqui abordados são desafiadores, mas a verificação de fenômenos não-estacionários em eletrodinâmica quântica de circuitos poderá forcenecer novas maneiras de geração de estados emaranhados por meio de termos contra-girantes no Hamiltoniano de interação luz-matéria.

Os resultados dessa dissertação foram publicados no artigo [44]. 


\section{Apêndice A}

\section{Operador Paridade}

Este apêndice tem como objetivo demonstrar que o operador paridade $\Pi=(-1)^{a^{\dagger} a+\sigma_{+} \sigma_{-}}$ [47], que define a paridade dos autoestados $|j\rangle$ do Hamiltoniano de Rabi, comuta com o Hamiltoniano e os seus autovalores rotulam paridade par ou ímpar.

Inicialmente, considere que $n=a^{\dagger} a$ e $E=\sigma_{+} \sigma_{-}$, então,

$$
\begin{aligned}
e^{-i \pi n} a e^{i \pi n} & =a e^{i \pi}=-a ; \\
e^{-i \pi n} a^{\dagger} e^{i \pi n} & =a^{\dagger} e^{-i \pi}=-a^{\dagger} ; \\
e^{-i \pi E} \sigma_{-} e^{i \pi E} & =\sigma_{-} e^{i \pi}=-\sigma_{-} ; \\
e^{-i \pi E} \sigma_{+} e^{i \pi E} & =\sigma_{+} e^{-i \pi E}=-\sigma_{+} .
\end{aligned}
$$

Logo,

$$
\begin{aligned}
a e^{i \pi n} & =-e^{i \pi n} a \rightarrow\left[a, e^{i \pi n}\right]=2 a e^{i \pi n} ; \\
a^{\dagger} e^{i \pi n} & =-e^{i \pi n} a^{\dagger} \rightarrow\left[a^{\dagger}, e^{i \pi n}\right]=2 a^{\dagger} e^{i \pi n} ; \\
\sigma_{-} e^{i \pi E} & =-e^{i \pi E} \sigma_{-} \rightarrow\left[\sigma_{-}, e^{i \pi E}\right]=2 \sigma_{-} e^{i \pi E} \\
\sigma_{+} e^{i \pi E} & =-e^{i \pi E} \sigma_{+} \rightarrow\left[\sigma_{+}, e^{i \pi E}\right]=2 \sigma_{+} e^{i \pi E} .
\end{aligned}
$$

Assim, o comutador $\left[a \sigma_{+}, \Pi\right]$ fica

$$
\begin{aligned}
{\left[a \sigma_{+}, \Pi\right] } & =\left[a \sigma_{+}, e^{i \pi n} e^{i \pi E}\right]=a e^{i \pi n}\left[\sigma_{+}, e^{i \pi E}\right]+\left[a, e^{i \pi n}\right] e^{i \pi E} \sigma_{+} \\
& =a e^{i \pi n} 2 \sigma_{+} e^{i \pi E}+2 a e^{i \pi n} e^{i \pi E} \sigma_{+} \\
& =2 a e^{i \pi n} e^{i \pi E}\left[e^{-i \pi E} \sigma_{+} e^{i \pi E}+\sigma_{+}\right]=0,
\end{aligned}
$$


enquanto para $\left[a^{\dagger} \sigma_{-}, \Pi\right]$ teremos

$$
\begin{aligned}
{\left[a^{\dagger} \sigma_{-}, \Pi\right] } & =\left[a^{\dagger} \sigma_{-}, e^{i \pi n} e^{i \pi E}\right]=a^{\dagger} e^{i \pi n}\left[\sigma_{-}, e^{i \pi E}\right]+\left[a^{\dagger}, e^{i \pi n}\right] e^{i \pi E} \sigma_{-} \\
& =a^{\dagger} e^{i \pi n} 2 \sigma_{-} e^{i \pi E}+2 a^{\dagger} e^{i \pi n} e^{i \pi E} \sigma_{-} \\
& =2 a^{\dagger} e^{i \pi n} e^{i \pi E}\left[e^{-i \pi E} \sigma_{-} e^{i \pi E}+\sigma_{-}\right]=0
\end{aligned}
$$

Os outros termos terão seus comutadores dados por

$$
\begin{aligned}
{\left[a \sigma_{-}, \Pi\right] } & =\left[a \sigma_{-}, e^{i \pi n} e^{i \pi E}\right]=a e^{i \pi n}\left[\sigma_{-}, e^{i \pi E}\right]+\left[a, e^{i \pi n}\right] e^{i \pi E} \sigma_{-} \\
& =a e^{i \pi n} 2 \sigma_{-} e^{i \pi E}+2 a e^{i \pi n} e^{i \pi E} \sigma_{-} \\
& =2 a e^{i \pi n} e^{i \pi E}\left[e^{-i \pi E} \sigma_{-} e^{i \pi E}+\sigma_{-}\right]=0
\end{aligned}
$$

e

$$
\begin{aligned}
{\left[a^{\dagger} \sigma_{+}, \Pi\right] } & =\left[a^{\dagger} \sigma_{+}, e^{i \pi n} e^{i \pi E}\right]=a^{\dagger} e^{i \pi n}\left[\sigma_{+}, e^{i \pi E}\right]+\left[a^{\dagger}, e^{i \pi n}\right] e^{i \pi E} \sigma_{+} \\
& =a^{\dagger} e^{i \pi n} 2 \sigma_{+} e^{i \pi E}+2 a^{\dagger} e^{i \pi n} e^{i \pi E} \sigma_{+} \\
& =2 a^{\dagger} e^{i \pi n} e^{i \pi E}\left[e^{-i \pi E} \sigma_{+} e^{i \pi E}+\sigma_{+}\right]=0 .
\end{aligned}
$$

Isto mostra que a relação de comutação realmente se anula e os autoestados do Hamiltoniano de Rabi têm paridade bem definida. 


\section{Apêndice B}

\section{Aproximação de onda girante (Rotating wave approximation-RWA)}

Na nossa análise sempre é possível reduzir as equações relevantes do sistema em duas equações diferenciais acopladas:

$$
\begin{aligned}
\frac{d}{d t} A(t) & =-q e^{i t W} B(t), \\
\frac{d}{d t} B(t) & =-q e^{-i t W} A(t) .
\end{aligned}
$$

A solução das equações diferencias é simples: basta que as equações sejam desacopladas e, em seguida, resolvidas em separado. Para isso, inicialmente, isolamos $A$ e $B$ (a variárel $t$ do tempo será omitida)

$$
\begin{aligned}
& A=-\frac{e^{i t W}}{q} \frac{d B}{d t} \\
& B=-\frac{e^{-i t W}}{q} \frac{d A}{d t},
\end{aligned}
$$

para depois derivá-las com relação ao tempo, obtendo:

$$
\frac{d}{d t} A=-i W \frac{e^{i t W}}{q} \frac{d B}{d t}-\frac{e^{i t W}}{q} \frac{d^{2} B}{d t^{2}}
$$

e

$$
\frac{d B}{d t}=i W \frac{e^{-i t W}}{q} \frac{d A}{d t}-\frac{e^{-i t W}}{q} \frac{d^{2} A}{d t^{2}} .
$$

Ao substituirmos as equações (B.1) e (B.2) nas equações anteriores, as equações serão desacopladas:

$$
\begin{aligned}
& \frac{d^{2} A}{d t^{2}}-i W \frac{d A}{d t}-q^{2} A=0 \\
& \frac{d^{2} B}{d t^{2}}+i W \frac{d B}{d t}-q^{2} B=0 .
\end{aligned}
$$


As soluções de equações diferenciais de segunda ordem são da forma $A=e^{\lambda t}$ e $B=e^{\lambda t}$, onde $\lambda$ é uma constante. Ao serem inseridas em (B.7) e (B.8) fornecem equações de segundo grau. Logo, para a substituição em $A$, teremos

$$
\lambda^{2}-i W \lambda-q^{2}=0
$$

com soluções

$$
\begin{aligned}
& \lambda_{1}=i(W+R) / 2, \\
& \lambda_{2}=i(W-R) / 2,
\end{aligned}
$$

onde $R=\sqrt{W^{2}-4 q^{2}}$. Então, $A$ pode ser escrita como uma combinação linearmente independente das soluções propostas:

$$
A=A_{1} e^{i t(W+R) / 2}+A_{2} e^{i t(W-R) / 2} .
$$

Analogamente, podemos chegar na solução para $B$

$$
B=B_{1} e^{-i t(W-R) / 2}+B_{2} e^{-i t(W+R) / 2}
$$

Para se obter os coeficientes de $A$, manipularemos algebricamente da seguinte forma

$$
A_{0}=\left(\frac{2 R}{2 R}\right) A_{0}
$$

e definiremos

$$
A_{0}=A_{1}+A_{2}
$$

Em seguida, fazemos a seguinte manipulação

$$
\begin{aligned}
A_{0} & =\left(\frac{2 R+W-W}{2 R}\right) A_{0}+\frac{2 q B_{0}-2 q B_{0}}{2 R} \\
& =\left[\frac{A_{0}(W+R)-2 q B_{0}}{2 R}\right]-\left[\frac{A_{0}(W-R)-2 q B_{0}}{2 R}\right] .
\end{aligned}
$$

Lembrando da condição $A_{0}=A_{1}+A_{2}$, definiremos

$$
A_{1}=\left[\frac{A_{0}(W+R)-2 i q B_{0}}{2 R}\right]
$$

e

$$
A_{2}=-\left[\frac{A_{0}(W-R)-2 i q B_{0}}{2 R}\right]
$$


para chegarmos à solução

$$
A(t)=e^{i t W / 2} \frac{-\left[(W-R) A_{0}-2 i q B_{0}\right] e^{i t R / 2}+\left[(W+R) A_{0}-2 i q B_{0}\right] e^{-i t R / 2}}{2 R} .
$$

Obtemos de forma análoga a solução

$$
B(t)=e^{-i t W / 2} \frac{\left[(W+R) B_{0}+2 i q A_{0}\right] e^{i R t / 2}-\left[(W-R) B_{0}+2 i q A_{0}\right] e^{-i R t / 2}}{2 R} .
$$

Impondo as condições $W^{2}-4 q^{2}>0$ e $|q| \ll|W|$, pode-se aproximar situações práticas em que $W$ é um termo de alta frequência em comparação com a taxa de acoplamento $q$. Assim, expandindo o termo $R$, teremos

$$
R \simeq|W|-\frac{2 q^{2}}{|W|}
$$

Nesse caso, as soluções para $A(t)$ e $B(t)$ podem ser aproximadas por

$$
\begin{aligned}
A(t) & \simeq \frac{1}{2 W}\left\{\frac{\left[2 W A_{0}-2 i q B_{0}\right] e^{i t q^{2} / W}+2 i q B_{0} e^{i t\left(W-\frac{2 q^{2}}{W}\right)}}{2 R}\right\} \\
& \simeq A_{0} e^{i t q^{2} / W}-\frac{2 q}{W} e^{i t W / 2} \sin (W t / 2) B_{0}
\end{aligned}
$$

analogamente

$$
B(t) \approx B_{0} e^{-i \frac{q^{2}}{W} t}-\frac{2 q}{W} e^{-i t W / 2} \sin (W t / 2) A_{0}
$$

Entretanto, a condição $|q| \ll|W|$ fornece as soluções aproximadas

$$
A \simeq A_{0,}, B \simeq B_{0}
$$

que podem ser pensadas em termos de suas derivadas temporais

$$
\frac{d A}{d t}=0 ; \frac{d B}{d t}=0
$$

É de suma importância enfatizar que toda vez que fazemos o RWA introduzimos incertezas nas frequências relevantes do sistema de ordem de $q^{2} / W$, que foram chamados de NFS (neglected frequency shift) em [43]. O conhecimento da ordem de magnitude destes shifts é importante na hora de ajustar as frequências ressonantes em simulações númericas.

O método RWA será útil sempre que for necessário eliminar termos com rápidas oscilações e sempre será cabível quando as condições forem as mesmas deste apêndice. 


\section{Apêndice C}

\section{Densidade Espectral}

Este apêndice tem como objetivo demonstrar as propriedades da densidade espectral da função randômica clássica $f(t)$ no termo de defasagem da Equação Mestra na representação dos Estados Vestidos. Indicamos a referência [46] para uma leitura mais aprofundada do tema. Inicialmente, faremos uma breve revisão de propriedades úteis sobre séries de Fourier e integrais. Se o leitor desejar, deixamos como indicação de leitura a referência [51] para maiores detalhes e exemplos conexos ao conteúdo.

Seja $x(t)$ uma função (real ou complexa) periódica de variável real $t$ (o tempo, por exemplo), se $x(t)$ é absolutamente integrável sobre o período $T$, isto é,

$$
\int_{0}^{T}|x(t)| d t<\infty,
$$

então $x(t)$ tem uma série de Fourier associada

$$
\hat{x}(t)=\sum_{n=-\infty}^{n=\infty} a_{n} e^{i n \omega_{0} t} ; \omega_{0}=\frac{2 \pi}{T}
$$

onde $a_{n}$ é o $n$-ésimo coeficiente da série de Fourier, que é dado por

$$
a_{n}=\frac{1}{T} \int_{0}^{T} x(t) e^{-i n \omega_{0} t} d t .
$$

Uma condição alternativa para $x(t)$ é que ela seja de quadrado integrável para $0 \leq t \leq T$, isto é, que 


$$
\int_{0}^{T}|x(t)|^{2} d t<\infty .
$$

Uma série uniformemente convergente de funções contínuas sempre resulta em uma função contínua (funções senoidais, por exemplo). Entretanto, algumas restrições garantem que a série de Fourier para a função $x(t)$ convergirá uniformemente [51]. A soma do lado direito de (C.2) converge para $x(t)$ no sentido que

$$
\lim _{N \rightarrow \infty} \int_{0}^{T}\left|x(t)-\sum_{n=-\infty}^{n=\infty} a_{n} e^{i n \omega_{0} t}\right|^{2} d t=0 .
$$

Isso significa que o limite do erro médio quadrático vai a zero e é conhecido como convergência na média, que nos permite escrever

$$
x(t)=\lim _{N \rightarrow \infty} \sum_{n=-N}^{N} a_{n} e^{i n \omega_{0} t} .
$$

Daqui em diante, assumiremos que a condição de (C.4) é sempre satisfeita para um número finito $T$, sendo $T$ o período de uma função periódica. A condição da equação (C.4) é requisito para que a energia em um período de $x(t)$ seja finita. Por conveniência, nós escreveremos $x(t)=\hat{x}(t)$.

Quando a condição (C.4) é satisfeita, um dos principais resultados que se pode obter é o teorema de Parseval

$$
\sum_{-\infty}^{\infty}\left|a_{n}\right|^{2}=\frac{1}{T} \int_{0}^{T}|x(t)|^{2} d t,
$$

que afirma que a energia média no sinal é igual a soma das energias médias em cada componente da frequência. Este teorema terá muita importância nas definições das próximas seções. Reforçamos que a prova rigorosa dessas afirmações pode ser encontrada em [51].

\section{C.1 Transformada de Fourier}

A transformada de Fourier $x(t)$ de uma função $X(f)$ é definida por

$$
x(t)=\int_{-\infty}^{\infty} X(f) e^{i \omega t} d f ; \omega=2 \pi f
$$

se a integral existe. A transformada inversa de Fourier de $x(t)$ é definida por 


$$
\hat{X}(f)=\int_{-\infty}^{\infty} x(t) e^{-i \omega t} d t ; \omega=2 \pi f
$$

se ela existe.

Um dos mais básicos resultados da teoria da Transformada de Fourier é o teorema de Plancherel, que afirma que se $X(f)$ é de quadrado-integrável em toda a linha, $-\infty<f<\infty$, isto é, se

$$
\int_{-\infty}^{\infty}|X(f)|^{2} d f<\infty
$$

então existe uma função $x(t)$ que também é de quadrado-integrável em toda a linha que é relacionada a $X(f)$ pelas equações

$$
\begin{aligned}
x(t) & =\lim _{A \rightarrow \infty} \int_{-A}^{A} X(f) e^{i \omega t} d f, \\
X(f) & =\lim _{A \rightarrow \infty} \int_{-A}^{A} x(t) e^{-i \omega t} d t .
\end{aligned}
$$

Sob essa condição das equações (C.11) e (C.12), um análogo do teorema de Parseval surge

$$
\int_{-\infty}^{\infty}|x(t)|^{2} d t=\int_{-\infty}^{\infty}|X(f)|^{2} d f .
$$

As equações (C.11) e (C.12) são conhecidas como um par Transformada de Fourier. Se $X(f)$ é absolutamente integrável, então $x(t)$ será dada por (C.8). Ainda, se $x(t)$ é absolutamente integrável, $X(f)=\hat{X}(f)$ será dada por (C.9).

\section{C.2 Densidade espectral de uma função periódica}

Nós agora introduziremos a função conhecida como densidade espectral de potência (ou simplesmente densidade espectral), que fornece a distribuição da frequência da potência de um sinal ou ruído, e sua relação com a função de autocorrelação.

Seja $x(t)$ uma função periódica de período $T$, que tem "energia" finita por período, isto é, que satifaz a equação (C.4). Então, pelo Teorema de Parseval a média temporal da energia (ou a potência) é igual a soma dos termos, cada termo associado com uma frequência na sua expansão em 
série de Fourier. Cada termo é interpretado como a média temporal da energia de um componente particular da frequência. Nós somos levados a definir uma função $S(f)$, a densidade espectral de potência, por

$$
S(f)=\sum_{-\infty}^{\infty}\left|a_{n}\right|^{2} \delta\left(f-n f_{0}\right) ; f=\frac{1}{T}
$$

onde $S(f)$ consiste de uma série de impulsos com as frequências componentes de $x(t)$ e é uma medida para a distribuição de potência em $x(t)$. A potência total em $x(t)$ é

$$
\int_{-\infty}^{\infty}|S(f)|^{2} d f=\sum_{-\infty}^{\infty}\left|a_{n}\right|^{2}=\frac{1}{T} \int_{0}^{T}|x(t)|^{2} d t
$$

O Espectro de Potência $G(f)$ de $x(t)$ é definido como

$$
G(f)=\int_{-\infty}^{f} S(\eta) d \eta
$$

O espectro de potência de uma função periódica é uma função escada com saltos a frequências harmônicas.

Agora será introduzida a função de autocorrelação $\Re(\tau)$ de uma função amostral de um processo randômico definida por

$$
\Re(\tau)=\lim _{T \rightarrow \infty} \frac{1}{2 T} \int_{-T}^{T} x(t+\tau) x^{*}(t) d t .
$$

No caso de uma função períodica com uma série de Fourier associada como na equação (C.4), tem-se

$$
\Re(\tau)=\lim _{T \rightarrow \infty} \frac{1}{2 T} \int_{-T}^{T}\left[\sum_{-\infty}^{\infty} a_{n}^{*} \exp \left(-i n \omega_{0} t\right)\right]\left\{\sum_{-\infty}^{\infty} a_{n} \exp \left[i n \omega_{0}(t+\tau)\right]\right\} d t .
$$

Ao trocarmos as ordens do somatório e da integral, além de usarmos o seguinte resultado

$$
\lim _{T \rightarrow \infty} \frac{1}{2 T} \int_{-T}^{T} \exp \left[-i(n-m) \omega_{0} t\right] d t=\lim _{T \rightarrow \infty} \frac{\sin \left[(n-m) \omega_{0} t\right]}{(n-m) \omega_{0} t}=1,
$$

se $m=n$, chegamos ao resultado

$$
\Re(\tau)=\sum_{-\infty}^{\infty}\left|a_{n}\right|^{2} \exp \left(i n \omega_{0} \tau\right)
$$

Se for tomada a Transformada de $\Re(\tau)$, o resultado será

$$
\begin{aligned}
\int_{-\infty}^{\infty} \Re(\tau) \exp (-i \omega \tau) d \tau & =\int_{-\infty}^{\infty} \sum_{-\infty}^{\infty}\left|a_{n}\right|^{2} \exp \left(i n \omega_{0} \tau\right) \exp (-i \omega \tau) d \tau \\
& =\sum_{-\infty}^{\infty}\left|a_{n}\right|^{2} \delta\left(f-n f_{0}\right)=S(f)
\end{aligned}
$$


Assim, para uma função periódica $x(t)$, a densidade espectral de potência e sua função de correlação formam um par Transformada de Fourier. Além disso, é interessante notar que em (C.14) toda a informação a respeito das fases das frequências presentes na expansão da série de Fourier de $x(t)$ são perdidas em $S(f)$. Por exemplo, duas funções $x(t)$ e $x^{\prime}(t)$ com os coeficientes de Fourier com mesma magnitude e fases diferentes terão a mesma densidade espectral.

\section{C.3 Densidade espectral de um processo randômico}

Um processo randômico estacionário com funções amostrais $x(t)$ é dito periódico com período $T$ se sua função de correlação $\Re(\tau)$ é periódica com período $T$. Um processo é dito estacionário se para qualquer $\epsilon, x(t+\epsilon)$ tem a mesma estatística que $x(t)$. Se todas as funções amostrais de um processo randômico são periódicas o processo é dito periódico. Indicamos a referência [46] para uma explicação mais completa sobre Variáveis randômicas e Distribuição de probabilidades.

Seja um processo periódico estacionário de período $T$ tendo funções amostrais $x(t)$. Suponha, por conveniência, que o valor médio $E\left(x_{t}\right)$ seja zero. Então, se as funções amostrais são periódicas e podem ser expandidas em série de Fourier, teremos

$$
x(t)=\sum_{n=-\infty}^{n=\infty} x_{n} e^{i n \omega_{0} t} ; \omega_{0}=\frac{2 \pi}{T},
$$

onde

$$
x_{n}=\frac{1}{T} \int_{0}^{T} x(t) e^{-i n \omega_{0} t} d t .
$$

Essas expressões são como as equações (C.2) e (C.3), mas agora os coeficientes de Fourier $x_{n}$ são variáveis randômicas no lugar de números.

Com as definições anteriores, é possível mostrar que o requisito para que o processo randômico seja periódico exige que $x_{n}$ e $x_{m}$ sejam descorrelacionados, com $n \neq m$. O valor médio de uma variável randômica é dado por

$$
\begin{aligned}
E\left(x_{n} x_{m}^{*}\right) & =\frac{1}{T^{2}} E\left[\int_{0}^{T} \int_{0}^{T} x(t) x^{*}(s) e^{-i n \omega_{0} t} e^{i \omega_{0} s} d s d t\right] \\
& =\frac{1}{T^{2}} \int_{0}^{T} \int_{0}^{T} R(t-s) e^{i \omega_{0}(m s-n t)} d s d t
\end{aligned}
$$

onde definimos $R(\tau)$ como

$$
R(\tau)=\sum_{n=-\infty}^{n=\infty} b_{n} e^{i n \omega_{0} t}
$$


sendo uma função periódica para todo $\tau$. Logo, substituindo $R(\tau)$ em (C.24), teremos

$$
\begin{aligned}
E\left(x_{n} x_{m}^{*}\right) & =\frac{1}{T^{2}} \int_{0}^{T} \int_{0}^{T} \sum_{k=-\infty}^{k=\infty} b_{k} e^{i k \omega_{0}(t-s)} e^{i \omega_{0}(m s-n t)} d s d t \\
& =\frac{1}{T^{2}} \sum_{k=-\infty}^{k=\infty} b_{k} \int_{0}^{T} e^{i \omega_{0}(m-k) s} d s \int_{0}^{T} e^{i \omega_{0}(k-n) t} d t\left\{\begin{array}{c}
b_{n}, m=n \\
0, m \neq n
\end{array} .\right.
\end{aligned}
$$

Esse resultado afirma não somente que $x_{n}$ e $x_{m}$ são descorrelacionados para $n \neq m$, mas também que $n$-ésimo coeficiente de Fourier da função de correlação $R(\tau)$ é igual a variância do $n$-ésimo coeficiente de Fourier de $x(t)$. Este fato é importante para definir o análogo estatístico para uma função periódica $x(t)$ (como na seção anterior). Assim, a energia em um sinal representado por um processo randômico é mais razoavelmente definida sendo a média estatística das energias nas funções amostrais. Então, temos para a energia em um sinal no intervalo de tempo $T$

$$
\begin{aligned}
E\left(\int_{-\infty}^{\infty}|x(t)|^{2} d t\right) & =E\left[\int_{0}^{T} \sum_{n=-\infty}^{n=\infty} x_{n} e^{i n \omega_{0} t} \sum_{n=-\infty}^{n=\infty} x_{n}^{*} e^{-i k \omega_{0} t} d t\right] \\
& =E\left[\sum_{n=-\infty}^{n=\infty}\left|x_{n}\right|^{2} T\right]=T \sum_{n=-\infty}^{n=\infty} b_{n},
\end{aligned}
$$

sendo a média temporal da energia em um sinal dada por $\sum_{n=-\infty}^{n=\infty} b_{n}$.

A densidade espectral de potência $S(f)$ para um processo estacionário periódico será definida como (analogamente ao definido na seção anterior)

$$
S(f)=\sum_{n=-\infty}^{n=\infty} b_{n} \delta\left(f-n f_{0}\right)
$$

onde

$$
b_{n}=\frac{1}{T} \int_{0}^{T} R(t) e^{-i n \omega_{0} t} d t .
$$

A transformada de Fourier da função de correlação é

$$
\begin{aligned}
\int_{-\infty}^{\infty} R(\tau) e^{-i n \omega_{0} \tau} d \tau & =\int_{-\infty}^{\infty} \sum_{n=-\infty}^{n=\infty} b_{n} e^{i n \omega_{0} \tau} e^{i \omega \tau} d \tau \\
& =\sum_{n=-\infty}^{n=\infty} b_{n} \delta\left(f-n f_{0}\right)=S(f)
\end{aligned}
$$

Como podemos observar, a densidade espectral é uma transformada de Fourier da função de autocorrelação. Este resultado trata-se do Teorema de Wiener-Khintchin. 


\section{Apêndice D}

\section{Estados Vestidos e autovalores de Jaynes-Cummings}

O Hamiltoniano que descreve a interação luz-matéria sob a aproximação de onda girante é o Hamiltoniano de Jaynes-Cummings (JC) [8]:

$$
\hat{H}_{J C}=\omega_{0} \hat{n}+\frac{\Omega_{0}}{2} \sigma_{z}+g_{0}\left(a \sigma_{+}+a^{\dagger} \sigma_{-}\right),
$$

onde $\omega_{0}$ é a frequência do campo, $\Omega_{0}$ é a frequência de transição atômica e $g_{0}$ é o termo de acoplamento átomo-campo. O termo $\hat{n}$ é o operador número $\left(a^{\dagger} a\right)$, os termos $a^{\dagger}$ e $a$ são os operados de criação e aniquilação. O operador do estado atômico é $\sigma_{z}$ e pode ser representado por meio dos operadores $\sigma_{+}$e $\sigma_{-}: \sigma_{z}=\sigma_{+} \sigma_{-}-\sigma_{-} \sigma_{+}[18]$. Sabe-se que este Hamiltoniano descreve fielmente a interação átomo-campo quando $g_{0} \ll \omega_{0}$.

O menor estado de energia pode ser obtido com a aplicação de (D.1) no estado de excitação atômica fundamental

$$
\hat{H}_{J C}|g, n\rangle=\left[\omega_{0} n-\frac{\Omega_{0}}{2}\right]|g, n\rangle+g_{0} \sqrt{n}|e, n-1\rangle,
$$

onde podemos obter para o estado fundamental

$$
\hat{H}_{J C}|g, 0\rangle=-\frac{\Omega_{0}}{2}|g, 0\rangle .
$$

Essa é a menor energia para o sistema, entretanto, iremos inserir esse valor como correção no Hamiltoniano (D.1) para que sua energia mínima seja

$$
\left[\hat{H}_{J C}+\frac{\Omega_{0}}{2}\right]|g, 0\rangle=0 .
$$


Assim, o autovalor para o estado de vácuo será nulo $\lambda_{0}=0$. A aplicação do Hamiltoniano de JaynesCummings no autoestado $|g, n\rangle$ forneceu uma superposição dos estados $|g, n\rangle$ e $|e, n-1\rangle$. Logo, escreveremos os autoestados de JC $\left|\varphi_{n}\right\rangle$ como

$$
\left|\varphi_{n}\right\rangle=\alpha|g, n\rangle+\beta|e, n-1\rangle,
$$

que seja normalizado

$$
\left|\left\langle\varphi_{n} \mid \varphi_{n}\right\rangle\right|^{2}=\alpha^{2}+\beta^{2}=1 .
$$

Observando os coeficientes na normalização do autoestados de JC, propomos que os autoestados sejam da forma

$$
\left|\varphi_{n,+}\right\rangle=\sin \theta_{n}|g, n\rangle+\cos \theta_{n}|e, n-1\rangle
$$

$\mathrm{ou}$

$$
\left|\varphi_{n,-}\right\rangle=\cos \theta_{n}|g, n\rangle-\sin \theta_{n}|e, n-1\rangle .
$$

Estados quânticos da forma (D.7) e (D.8) são conhecidos na literatura como Estados Vestidos (tradução para dressed states) por englobarem descrição sobre a interação entre átomo e campo dentro de um único estado.

A notação pode ser compactada da seguinte maneira

$$
\begin{gathered}
\left|\varphi_{0}\right\rangle=|g, 0\rangle, \\
\left|\varphi_{n, \mathcal{S}}\right\rangle=\left(\mathrm{s}_{n, \mathcal{S}}|g, n\rangle+\mathrm{c}_{n, \mathcal{S}}|e, n-1\rangle\right), \\
\mathrm{s}_{n,+}=\sin \theta_{n}, \\
\mathrm{~s}_{n,-}=\cos \theta_{n}, \\
\mathrm{c}_{n,+}=\cos \theta_{n}, \\
\mathrm{c}_{n,-}=-\sin \theta_{n} .
\end{gathered}
$$

O símbolo $\mathcal{S}= \pm$ é um símbolo mudo que serve para diferenciar estados com mesmo número de excitações, que podem ser invertidos com uma álgebra simples

$$
\begin{aligned}
|g, n\rangle & =\sin \theta_{n}\left|\varphi_{n,+}\right\rangle+\cos \theta_{n}\left|\varphi_{n,-}\right\rangle, \\
|e, n-1\rangle & =\cos \theta_{n}\left|\varphi_{n,+}\right\rangle-\sin \theta_{n}\left|\varphi_{n,-}\right\rangle .
\end{aligned}
$$


Seus autovalores podem ser obtidos diretamente pela aplicação do Hamiltoniano JC nos EV

$$
\hat{H}_{J C}\left|\varphi_{n, \mathcal{S}}\right\rangle=\lambda_{n, \mathcal{S}}\left|\varphi_{n, \mathcal{S}}\right\rangle
$$

Começando com $\mathcal{S}=+$, a equação de autovalor será

$$
\hat{H}_{J C}\left|\varphi_{n,+}\right\rangle=\lambda_{n,+} \sin \theta_{n}|g, n\rangle+\lambda_{n,+} \cos \theta_{n}|e, n-1\rangle
$$

$\mathrm{ou}$

$$
\begin{aligned}
\hat{H}_{J C}\left|\varphi_{n,+}\right\rangle= & \left(\omega_{0} n \sin \theta_{n}+g_{0} \sqrt{n} \cos \theta_{n}\right)|g, n\rangle \\
& +\left[g_{0} \sqrt{n} \sin \theta_{n}+\left(\omega_{0}(n-1)+\Omega_{0}\right) \cos \theta_{n}\right]|e, n-1\rangle .
\end{aligned}
$$

A comparação entre as duas equações fornece, após uma álgebra simples, o resultado

$$
\cos \theta_{n}=\frac{\sin \theta_{n}\left(\lambda_{n,+}-\omega_{0} n\right)}{g_{0} \sqrt{n}}
$$

e

$$
\cos \theta_{n}=\frac{g_{0} \sqrt{n} \sin \theta_{n}}{\lambda_{n,+}-\omega_{0}(n-1)-\Omega_{0}},
$$

que ao serem igualadas e resolvidas para $\lambda_{n,+}$, após uma pequena álgebra, teremos

$$
\lambda_{n,+}=\frac{-\Delta_{-}+2 \omega_{0} n \pm \sqrt{\Delta_{-}^{2}+4 g_{0}^{2} n}}{2},
$$

onde $\Delta_{-}=\omega_{0}-\Omega_{0}$ é o termo de dissintonia. Introduzindo a definição $\beta_{n}=\sqrt{\Delta_{-}^{2}+4 g_{0}^{2} n}$, as soluções para o autovalor $\lambda_{n,+}$ serão

$$
\lambda_{n,+}=\omega_{0} n-\frac{\Delta_{-}}{2} \pm \frac{1}{2} \beta_{n} .
$$

Escolhendo o sinal + , a solução de $\lambda_{n,+}$ será

$$
\lambda_{n,+}=\omega_{0} n-\frac{\Delta_{-}}{2}+\frac{1}{2} \beta_{n} .
$$

O autovalor $\lambda_{n,-}$ pode ser obtido de maneira análoga, então

$$
\lambda_{n,-}=\omega_{0} n-\frac{\Delta_{-}}{2}-\frac{1}{2} \beta_{n} .
$$

Esses autovalores podem ser generalizados novamente pelo símbolo $\mathcal{S}$

$$
\lambda_{n, \mathcal{S}}=\omega_{0} n-\frac{\Delta_{-}}{2}+\mathcal{S} \frac{1}{2} \beta_{n} .
$$


Além disso, podemos manipular $\cos \theta_{n}=\frac{\sin \theta_{n}\left(\lambda_{n,+}-\omega_{0} n\right)}{g_{0} \sqrt{n}}$ de tal forma que obteremos

$$
\tan \theta_{n}=\frac{g_{0} \sqrt{n}}{\left(\lambda_{n,+}-\omega_{0} n\right)}=\frac{2 g_{0} \sqrt{n}}{\beta_{n}-\Delta_{-}}
$$

ou, após nova álgebra, o ângulo

$$
\theta_{n}=\arctan \frac{\beta_{n}+\Delta_{-}}{2 g_{0} \sqrt{n}}
$$




\section{Bibliografia}

[1] A. Blais, R.-S. Huang, A. Wallraff, S. M. Girvin, and R. J. Schoelkopf, "Cavity quantum eletrodynamics for superconducting electrical circuits: An architecture for quantum computation", Phys. Rev. A 69, 062320 (2004).

[2] R. J. Schoelkopf and S. M. Girvin, "Wiring up quantum systems", Nature 451, 664-669, 1031 (2008).

[3] A. Wallraf, D. I. Schuster, A. Blais, L. Funzio, R.-S. Huang, J. Majer, S. Kumar, S. M. Girvin, and R. J. Schoelkopf, "Strong coupling of a single photon to a superconducting qubit using circuit quantum electrodynamics", Nature 431, 162-167 (2004).

[4] J. Clarke and F. K. Willhelm, "Superconducting quantum bits", Nature 453, 1031-1042 (2008).

[5] A. V. Dodonov and V. V. Dodonov, "Dynamical Casimir effect in a cavity with an N-level detector or N-1 two-level atoms", Phys. Rev. A 86, 015801 (2012).

[6] A. A. Houck, J. A. Schreier, B. R. Johnson, J. M. Chow, Jens Koch, J. M. Gambetta, D. I. Schuster, L. Frunzio, M. H. Devoret, S. M. Girvin, and R. J. Schoelkopf, "Controlling the Spontaneous Emission of a Superconducting Transmon Qubit", Phys. Rev. Lett. 101, 080502 (2008).

[7] S. De Liberato, C. Ciuti, and I. Carusotto, "Quantum vacuum radiation spectra from a semiconductor microcavity with a time-modulated vacuum Rabi frequency", Phys. Rev. Lett. 98, 103602 (2007).

[8] E. T. Jaynes, F. W. Cummings, "Comparison of quantum and semiclassical radiation theories with application to the beam maser", Proc. IEEE 51, 89-109 (1963). 
[9] G. J. Milburn, "Coherence and Chaos in a quantum optical system", Phys. Rev. A 41, 65-67 (1990).

[10] Jay Gambetta, Alexandre Blais, D. I. Schuster, A. Wallraff, L. Frunzio, J. Majer, M. H. Devoret, S. M. Girvin, and R. J. Schoelkopf, "Qubit-photon interactions in a cavity: Measurement-induced dephasing and number splitting", Phys. Rev. A 74, 042318 (2006).

[11] M. Brune, E. Hagley, J. Dreyer, X. Maître, A. Maali, C. Wunderlich, J. M. Raimond, and S. Haroche, "Observing the progressive decoherence of the "Meter" in a quantum measurement", Phys. Rev. Lett. 77, 4887 (1996).

[12] A. V. Dodonov, R. Lo Nardo, R. Migliore, A. Messina and V. V. Dodonov, "Analytical and numerical analysis of the atom-field dynamics in non-stationary cavity QED", J. Phys. B 44, 225502 (2011).

[13] J. M. Raimond, M. Brune, and S. Haroche, "Manipulating quantum entanglement with atoms and photons in a cavity", Rev. Mod. Phys. 73, 565 (2001).

[14] L. Landau, "The damping problem in wave mechanics", Z. Phys. 45, 430 (1927).

[15] W. Pauli, in Probleme der modern Physik, Arnold Sommerfeld zum 60. Geburtstag, edited by P. Debye (Hyrzel, Leipzig, 1928), p. 30.

[16] F. Beaudoin, J. M. Gambetta, and A. Blais, "Dissipation and ultrastrong coupling in circuit QED", Phys. Rev. A 84, 043832 (2011).

[17] I. S. Osad'ko, "Sub- and super-poissonian photons statistics os single molecule fluorescence blinking", J. Exp. Theor. Physics 104, 839-853 (2007).

[18] R. R. Puri, Mathematical Methods of Quantum Optics (Springer, Berlin, 2001).

[19] W. Vogel and D.-G. Welsh, Quantum Optics (Wiley-VCH, Weinheim, 2006).

[20] H. Carmichael, An Open Systems Approach to Quantum Optics (Springer, Berlin, 1993).

[21] G. Lindblad, "On the generators of Quantum Dynamical Semigroups", Commun. Math. Phys. 48, 119-130 (1976). 
[22] V. Gorini, A. Kossakowski, E. C. G. Sudarshan, "Completely positive dynamical semigroups os N-level systems", J. Math. Phys. 17, 821 (1976).

[23] H.-P. Breuer and F. Petruccione, The Theory of Quantum Systems (Oxford University Press, Oxford, 2002).

[24] C. W. Gardner and P. Zoller, Quantum Noise (Springer, Berlin, 2000).

[25] G. Benenti, S. Siccardi, G. Strini, "Exotic states in the dynamical Casimir effect", Eur. Phys. J. D 68, 139 (2014).

[26] S. De Liberato, D. Gerace, I. Carusotto, and C. Ciuti, "Extracavity quantum vacuum radiation from a single qubit", Phys. Rev. A 80, 053810 (2009).

[27] I. Carusotto, S. De Liberato, D. Gerace, and C. Ciuti, "Back-reaction effects of quantum vacuum in cavity quantum electrodynamics", Phys. Rev. A 85, 023805 (2012).

[28] N. G. Van Kampen, Stochastics Process in Physics and Chemistry (Elsevier Science, Amsterdam, 1992).

[29] L. Accardi and K. Imafuku, "Dynamical detailed balance and local KMS condition for nonequillibrium states", Int. J. Mod. Phys. B 18, 435 (2004).

[30] A. V. Dodonov, S. S. Mizrahi, and V. V. Dodonov, "Quantum master equations from classical Lagrangians with two stochastic forces", Phys. Rev. E 75, 011132 (2007).

[31] T. Werlang, A. V. Dodonov, E. I. Duzzioni, and C. J. Villas-Bôas, "Rabi model beyond rotatingwave approximation: Generation of photons from vacuum through decoherence", Phys. Rev. A 78, 053805 (2008).

[32] C. K. Law, "Effective Hamiltonian for the radiation in a cavity with a moving mirror and a time-varying dielectric medium", Phys. Rev. A 49, 433 (1994).

[33] T. Fujii, S. Matsuo, N. Hatakenaka, S. Kurihara, and A. Zeilinger, "Quantum circuit analog of the dynamical Casimir effect", Phys. Rev. B 84, 174521 (2011). 
[34] G. Kirchmair, B. Vlastakis, Z. Leghtas, S. E. Nigg, H. Paik, E. Ginossar, M. Mirrahimi, L. Frunzio, S. M. Girvin, and R. J. Schoelkopf, "Observation of quantum state collapse and revival due to the single-photon Kerr effect", Nature 495, 205-209 (2013).

[35] N. B. Narozhny, A. M. Fedotov, and Yu. E. Lozovik, "Dynamical Lamb effect versus dynamical Casimir effect", Phys. Rev. A 64, 053807 (2001).

[36] C. M. Wilson, G. Johansson, A. Pourkabirian, M. Simoen, J. R. Johansson, T. Duty, F. Nori, and P. Delsing, "Observation of the dynamical Casimir effect in a superconducting circuit", Nature 479, 376-379 (2011).

[37] H. Mabuchi and A. C. Doherty, "Cavity Quantum Electrodynamics: Coherence in Context", Nature 298, 1372 (2002).

[38] D. I. Schuster, Circuit Quantum Electrodynamics, tese de doutorado (2007).

[39] P. Lätheenmäki, G. S. Paraoanu, J. Hassel, and P. J. Hakonen, "Dynamical Casimir effect in a Josephson metamaterial", Proc. Nat. Acad. Sci. 110, 4234 (2013).

[40] M. Scala, B. Militello, A. Messina, S. Maniscalco, J. Piilo and K.-A. Suominen, "Cavity losses for the dissipative Jaynes-Cummings Hamiltonian beyond rotating wave approximation", J. Phys. A: Math. and Theor. 40, 14527 (2007).

[41] A. V. Dodonov, "Photon creation from vacuum and interactions engineering in nonstationary circuit QED", J. Phys.: Conf. Ser. 161, 012029 (2009).

[42] A. V. Dodonov, "Analytical description os nonstationary circuit QED in the dressed-state basis", J. Phys A: Math. and Theor. 47, 285303 (2014).

[43] I. M. de Sousa and A. V. Dodonov, "Microscopic toy model for the cavity dynamical Casimir effect", J. Phys A: Math. and Theor. 48, 245302 (2015).

[44] D. S. Veloso and A. V. Dodonov, "Prospects for observing dynamical and anti-dynamical Casimir effects in circuit QED due to fast modulation of qubit parameters", J. Phys. B: At. Mol. Opt. Phys. 48, 165503 (2015). 
[45] M. H. Devoret. S. Girvin, and R. Schoelkopf, "Circuit QED: How strong can the coupling between a Josephson junction atom and a transmition line resonator be?", Ann. Phys. 16, 767 (2007).

[46] W. B. Davenport Jr, W. L. Root, An Introduction to the Theory of Random Signals and Noise (Wiley, New York, 1987).

[47] W. H. Louisell, Quantum Statistical Properties of Radiation (Wiley, New York, 1973).

[48] D. Braak, "Integrability of the Rabi model", Phys. Rev. Lett. 107, 100401 (2011).

[49] I. M. de Sousa, "Formulação analítica de modulações temporais em Eletrodinâmica Quântica de Circuitos: Um Toy Model para o efeito Casimir dinâmico e novas aplicações", Dissertação de mestrado (2015).

[50] A. V. Dodonov, L. C. Céleri, F. Pascoal, M. D. Lukin, and S. F. Yelin, "Photon generation from vacuum in non-stationary circuit QED", arxiv: 0806.4035 (2008).

[51] J. W. Brown and R. V. Churchill, Fourier Series and Boundary Value Problems (McGraw-Hill, inc., New York, 1993). 